\title{
Valves in the Heart of the Big Apple VIII: Evaluation and Management of Valvular Heart Diseases 2014
}

Fifth Annual Joint Scientific Session of the Heart Valve Society of America and Society of Heart Valve Diseases, New York City, N.Y., May 8-10, 2014

\section{Abstracts}




\title{
Valves in the Heart of the Big Apple VIII: Evaluation and Management of Valvular Heart Diseases 2014
}

\author{
Fifth Annual Joint Scientific Session of the Heart Valve Society of America \\ and Society of Heart Valve Diseases, New York City, N.Y., May 8-10, 2014
}

\section{Early Outcomes After Aortic Valve Replacement, in 906 Patients, Older Than 70 Years of Age - The Brazilian Aortic Valve Replacement Study (BRAVARS)}

\author{
Rui M.S. Almeida' ${ }^{1}$, João Carlos Leal' ${ }^{2}$ Fernando Moraes ${ }^{3}$, \\ Eduardo K. Saadi ${ }^{4}$, Renato A. Kalil ${ }^{5}$, Orlando Petrucci ${ }^{6}$, \\ Gustaco C. Ribeiro ${ }^{7}$, Ricardo Sgarbieri ${ }^{8}$, Leonardo A. Mulinari ${ }^{9}$, \\ Fernando Pivatto Jr. ${ }^{5}$, Diogo Feraz ${ }^{3}$, Cledicyon E. Costa ${ }^{7}$, \\ Pedro P. Oliveira ${ }^{6}$, Kelter J. Sgobi ${ }^{8}$, Christian Hahn ${ }^{9}$ \\ ${ }^{1}$ Unioeste, Cascavel, Brazil; ${ }^{2}$ FAMERP, Sao Jose do Rio Preto, \\ Brazil; ${ }^{3} U F P E$, Recife, Brazil; ${ }^{4}$ UFRGS, Porto Alegre, Brazil; \\ ${ }^{5}$ Instituto de Cardiologia, Porto Alegre, Brazil; ${ }^{6}$ Unicamp, \\ Campinas, Brazil; ${ }^{7}$ PUC-Campinas, Campinas, Brazil; ${ }^{8}$ UFTM, \\ Uberaba, Brazil; ${ }^{9}$ UFPr, Curitiba, Brazil
}

$23 \mathrm{~mm}$ in $42.39 \%$ of the group. The mean cross clamp and bypass time were, respectively $57.60 \pm 19.16$ and $72.59 \pm 22.85$ minutes. The length of stay at ICU was $4.38 \pm 6.27$ days and the length of stay in the hospital was $11.02 \pm 11.66$ days. The hospital mortality, considering 30 days, was $4.63 \%$, being sepsis and cardiogenic shock the most frequently causes of death. A sub analyses on patients older than 80 years $(n=206)$ with a mean age of $82.66 \pm 2.55$ years was performed, with no statistical difference from the all group. The OR for variables age, sex, bypass time and ES were calculated. Risk factor for death, by logistic regression were ES, above $10 \%$ ( $\mathrm{p}<$ $0.01)$ and by-pass time $(p=0.02)$. Conclusions: Conventional AVR, in patients above 70 years of age, is safe with an acceptable risk for the overall group, and the depending variables found were ES and bypass time. These data should be the golden standard for any type of procedure, such as TAVI, and should be taken into account when discussing between Heart Team.

Objective: Transcatheter aortic valve implantation (TAVI) become an established intervention, for management of high-risk patients with aortic stenosis. Despite the fact that the Heart Team is mandatory to decide which type of treatment should be performed in a certain patient, a comparison of results, between techniques, should be done. To stratify patients' morbidity and mortality, patients, older than 70 years, who underwent isolated aortic valve replacement (AVR), by conventional bypass technique, were evaluated. Methods: A retrospective cohort study, between Jan/2001 and Dec/2011, in patients who underwent AVR from nine Brazilian centers, enrolling a total of 906 patients. All the data was collected in one center was stored in one center (RMSA) and analyzed in another (JCL), being this analysis blind. Results: The mean age was $75.93 \pm 4.60$ years, being $53.86 \%$ men. The estimated mortality ranged from 5.87 to $40.57 \%$ calculated with EuroScore II (ES) index. The surgery was elective in $96.48 \%$ and biological prostheses were used in 894 patients, due to age and patients' choice. The valve diameter most frequently implanted was that of number

\section{D Printer-Based Molding for the Preparation of Biovalve Family in In-Body Tissue Architecture Technology}

Yasuhide Nakayama ${ }^{1}$, Yoshiaki Takewa ${ }^{1}$, Masami Uechi ${ }^{2}$, Keiichi Kanda ${ }^{3}$, Takeshi Moriwaki' ${ }^{1}$,Tomonori Oie ${ }^{4}$, Takaharu Tanaka ${ }^{5}$, Hisashi Sugiura ${ }^{5}$

${ }^{1}$ National Cerebral and Cardiovascular Center Research Institute, Osaka, Japan; ${ }^{2}$ Nihon University, Kanahgawa, Japan;

${ }^{3}$ Kyoto Prefectural University of Medicine, Kyoto, Japan;

${ }^{4}$ Shinkan Co, Osaka, Japan; ${ }^{5}$ Goodman Co, Nagoya, Japan

Purpose: Our in body tissue architecture technology, as novel and practical regeneration medicine, can prepare completely autologous heart valves, called Biovalves, consisting of only recipi- 
ent's own tissue according to the shape of mold faithfully. In this study, 3D printer was used for design of the preparation molds for Biovalve family and valvular function was evaluated in vitro and in vivo. Methods and Results: $3 \mathrm{D}$ printers (Projet or Objet) could reproduce easily the $3 \mathrm{D}$-shape and size of native heart valves regardless of types within several hours. Only 1-month subcutaneous embedding of the assembling of 2 conduit parts and 3 sinus parts produced aortic or pulmonary valve-shaped Biovalves from completely autologous connective tissue with collagen and fibroblasts. As an aortic valve Biovalve in vitro evaluation using a pulsatile circulation circuit showed excellent valvular functions. Mean flow was maintained up to 10 days in the saline solution at $37^{\circ} \mathrm{C}$ with high durability. Upon implantation of the Biovalves in a beagle or a goat model good valvular function was obtained for 6 months. Combination with stents (Goodman Co.) at the mold embedding formed stent-impregnated Biovalves. By catheter-induced implantation of the Biovalves TAVI in a goat model or TPVI in a canine model were performed. In addition, mitral-type and tricuspid-type Biovalves were similarly formed by 3D molding in body. Their leaflets and tendinous cords were connected robustly and seamlessly. In a canine model, after surgical replacement postoperative echocardiography showed smooth movement of the leaflets with little regurgitation under systemic circulation. In all implantation study, the luminal surface after implantation was very smooth and fully covered with thin neointima including endothelial cells without thrombus formation. Conclusion: Functional, autologous, 3D-shaped, aortic, pulmonary, mitral, and tricuspid valves with clinical application potential were formed by only in body embedding of specially designed molds, which could be prepared by $3 \mathrm{D}$ printer within several hours.

\section{Long Term Evaluation of In-Body Tissue Engineered Heart Valve (Biovalve)}

Yoshiaki Takewa

National Cerebral and Cardiovascular Center, Osaka, Japan

Objective: A novel autologous aortic valve with a metallic stent (Biovalve Stent) was developed, using simple, safe and economical in-body tissue engineering. In this study, the long-term evaluation of the Biovalve Stent for transcatheter implantation was investigated in a goat model. Methods: Biovalve Stents were prepared by 2 -month embedding of the molds, assembled using plastic rods and a metallic stent, in the subcutaneous spaces of goats. After extracting the molds and removing the plastic rods only, Biovalve Stents with tri-leaflets similar to those of the native aortic valves were constituted from completely autologous connective tissues. Twelve out of 15 Biovalve Stents were implanted in the aorta in situ and other 3 Biovalve Stents were implanted in the pulmonary artery (PA) in situ with transcatheter technique. Results: In both aortic and PA cases, the Biovalve Stents were successfully implanted. Angiography showed smooth movement of the leaflets with a little regurgitation under the systemic and pulmonary circulation. The Biovalve Stents were extracted 1, 2, 5 or 6 months after implantation. The leaflets of the Biovalve kept their shape and elasticity even after 5 months and neither calcification nor thrombi were observed. Histological examination showed the cell populations inside the valves and endothelial cells covering the laminar surface of the valve leaflets. Conclusions: The Biovalve Stent satisfied the higher requirements of systemic and pulmonary circulation in goats for maximum 6 months with the potential for transcatheter implantation.

\section{Role of Matrix Metalloproteinas in Complications of Thoracic Aorta Aneurysm}

\author{
Calogera Pisano, Carmela Rita Balistreri, Tommaso Delisi, \\ Salvatore Ocello, Gianfranco Filippone, Daniela Buono, \\ Ugo Di Blasi, Maria Concetta Guarneri, Oreste Fabio Triolo, \\ Vincenzo Argano, Cesira Palmeri, Giovanni Ruvolo \\ University of Palermo, Palermo, Italy
}

Objective: Matrix metalloproteinases (MMPs) are endopeptidases involved in extra-cellular matrix remodelling, associated with both physiological and pathological processes of several human tissues and systems, such as vascular system. It is well known their involvement in mediating both beneficial and pathological aorta effects, such as abdominal aorta aneurysms and its complications. On the contrary, unclear data exist about their role in the pathophysiology of sporadic thoracic aorta aneurysm (TAA) and its complications. Thus, the aim of this study was to analyse the role of MMPs in TAA complications, i.e. rupture and dissection. Methods: Aortic specimens obtained from 73 patients $(51$ men and 22 women, age $61.7 \pm 10.7$ years) affected by TAAs, 18 patients with type A aortic dissection (TAD) and 30 controls were utilised for histo-pathological and immune-histochemical analyses. In addition, a second control group of 128 subjects (61 men and 67 woman, age $61.1 \pm 5.8$ years) was enrolled to examine the role of single nucleotide polymorphisms (SNPs) of MMP-9 (NM-004985), MMP-2 (NM-001121363.1) genes in diseases risk. Results: Three different patterns of MMPs (extracellular, intracellular and mixed) with different concentration (low, moderate, elevated) have been observed in case aorta samples. The pattern with elevated MMP amount in aorta samples from TAD cases was also characterised by increased cystic medial degeneration, without substitutive fibrosis, and plurifocal medial apoptosis. In the context of TAA aorta samples, we identified three phenotypes: phenotype I (normal wall); phenotype II (moderate wall thickness); phenotype III (thin and weak wall). In particular TAA phenotype III mainly observed in case samples showed the same histological features of TAD with elevated MMP concentration with a mixed pattern. In addition, significant associations were observed between the $1562 \mathrm{C} / \mathrm{T}$ MMP-9 and -735C/T MMP-2 SNPs and the risk of both TAA and TAD. Conclusions: Our data suggest a crucial role of both MMP2 and MMP-9 in both TAA and its complications, such as TAD. In future they might be considered as new criteria in TAA surgical indications. 


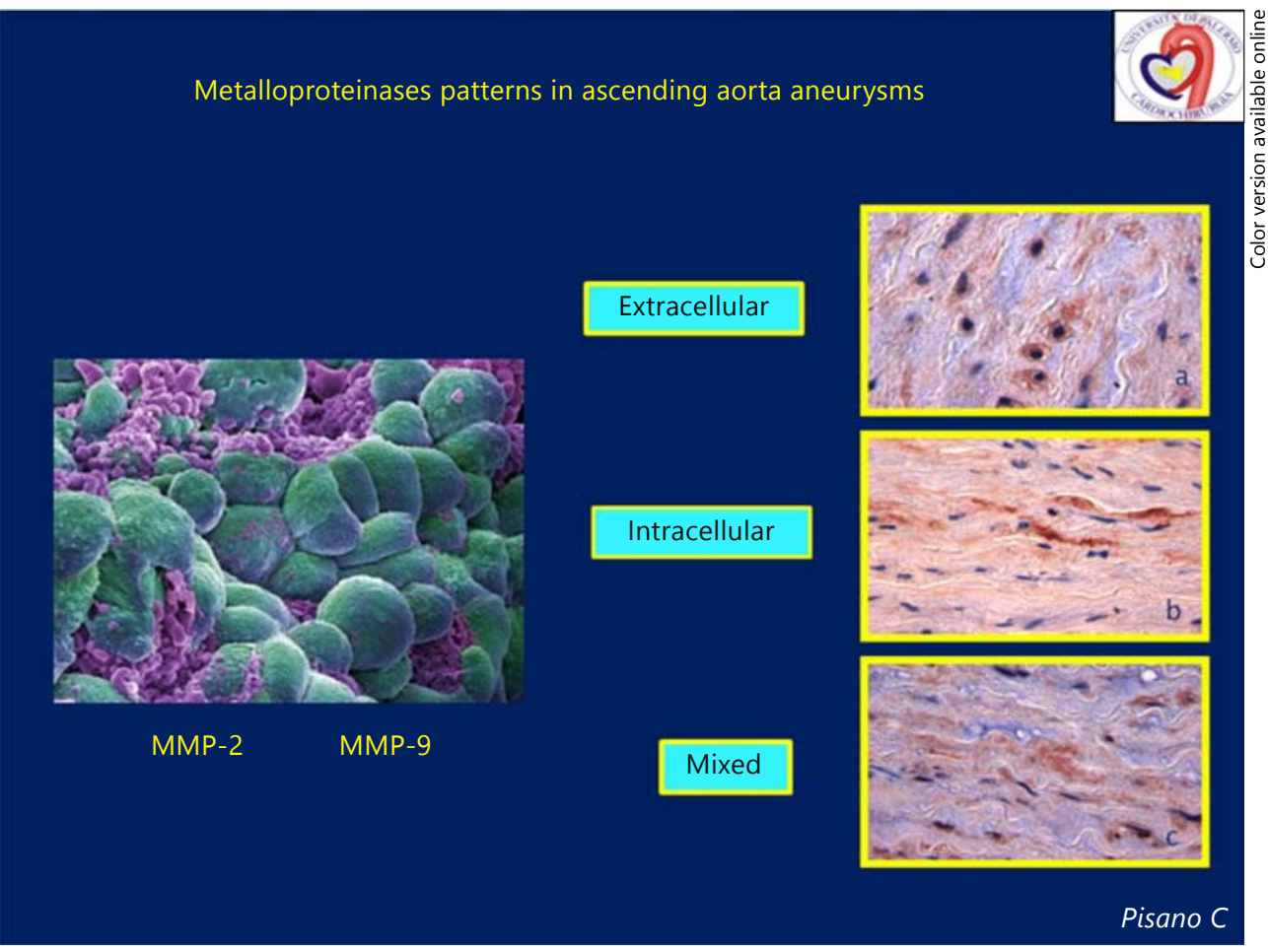
sano).

\section{Mitral Annuloplasty Does Not Improve Survival in Patients Having Moderate Ischemic Mitral Regurgitation Undergoing CABG}

\section{Shuli Silberman, Atia Alshousha, Daniel Fink, Rachel Tauber, Daniel Bitran, Ofer Merin}

Shaare Zedek Medical Center, Jerusalem, Israel

Background: The best surgical approach for patients with moderate mitral regurgitation of ischemic etiology (IMR) is still undetermined. We examined long term outcomes in patients undergoing coronary bypass (CABG) and having moderate IMR, and compared outcomes between those undergoing a restrictive annuloplasty to those not having intervention on the mitral valve. Methods: A retrospective analysis of our data base revealed 231 patients operated between 1993-2011 with moderate IMR: group 1 ( $\mathrm{n}=$ 186) underwent isolated CABG, group $2(n=45)$ underwent CABG with concomitant mitral valve annuloplasty. Univariate analysis was used to compare baseline parameters. Kaplan-Meier estimates were used to compare survival. Cox multivariate regression was used to determine predictors for late survival. Propensity matching yielded 59 and 38 patients in groups 1 and 2 respectively. Survival data up to 20 years is $97 \%$ complete. Results: The groups were similar with respect to preoperative baseline parameters including age, prior MI, LV function, and incidence of atrial fibrillation. Patients undergoing mitral repair had a higher incidence of CHF $(p<0.0001)$. After surgery more repair patients had low cardiac output $(\mathrm{p}=0.002)$ and required use of inotropes $(\mathrm{p}=$ 0.0005 ). Overall operative mortality was $7 \%$ and similar between groups. Ten year survival was $55 \%$ and $52 \%$ for groups 1 and 2 re- spectively $(p=0.2)$. In both the complete cohort as well as the matched patients, predictors for late mortality included age, symptoms of CHF, LV dimensions and preoperative LV function. Neither the addition of a mitral procedure, type of ring implanted nor residual MR after surgery emerged as predictors for survival. Conclusions: In patients with ischemic MR of moderate degree, neither operative mortality nor long term survival are affected by the performance of a restrictive annuloplasty. The fact that patients undergoing a mitral procedure had a higher incidence of CHF, yet they attained similar survival may support the notion that such a procedure is beneficial in terms of survival. Therefore the decision to repair the valve should be taken on an individual basis.

\section{Outcomes of a Hybrid Approach of Proximal Left Anterior Descending Coronary PCI Followed by Minimally Invasive Valve Surgery}

\author{
Andres M. Pineda, Orlando Santana, Christos G. Mihos, \\ Mery J. Cortes-Bergoderi, Nirat Beohar, Gervasio A. Lamas, \\ Joseph Lamelas
}

Mount Sinai Heart Institute, Miami, FL, USA

Objective: A sub-set of patients requiring coronary revascularization of the proximal left anterior descending (LAD) coronary artery and valve surgery may benefit from a hybrid approach of percutaneous coronary intervention (PCI) followed by minimally invasive valve surgery (MIVS), rather than combined median sternotomy 
Table 1. (for Abstract of Andres M. Pineda)

\begin{tabular}{lc}
\hline Patients Baseline Characteristics & $\mathrm{N}=59$ \\
\hline Age (years, mean \pm SD) & $75.3 \pm 8.8$ \\
Male gender (\%) & $41(69.5)$ \\
Hypertension (\%) & $56(94.9)$ \\
Diabetes mellitus (\%) & $20(33.9)$ \\
Cerebrovascular disease (\%) & $8(13.6)$ \\
Peripheral vascular disease (\%) & $7(11.9)$ \\
Ejection fraction (\%, median, IQR) & $55(45-63)$ \\
Pre-operative creatinine (mg/dl, median, IQR) & $1.0(0.9-1.3)$ \\
\hline Percutaneous Coronary Intervention (PCI) Characteristics \\
\hline Drug eluting stent (\%) & $45(76.3)$ \\
Bare metal stent (\%) & $14(23.7)$ \\
Single-vessel PCI (\%) & $45(76.3)$ \\
Dual-vessel PCI (\%) & $14(23.7)$ \\
Triple-vessel PCI (\%) & $0(0)$ \\
Left circumflex coronary artery (\%) & $10(16.9)$ \\
Right coronary artery (\%) & $3(5.1)$ \\
PCI to valve surgery time (days, median, IQR) & $39(10-57)$ \\
Pre-operative dual anti-platelet therapy (\%) & $45(76.3)$ \\
\hline Minimally Invasive Valve Surgery (MIVS) Characteristics
\end{tabular}

Cardiopulmonary bypass time (min, median, IQR)

Aortic cross clamp time (min, median, IQR)

Patients requiring intra-operative packed red blood cells (\%)

Aortic valve replacement (\%)

Mitral valve replacement (\%)

Mitral valve repair (\%)

Single valve (\%)

Double valve (\%)

Re-operation (\%)

Conversion to median sternotomy (\%)

$108(90-133)$

$81(67-102)$

$32(54.2)$

$38(64.4)$

$18(30.5)$

$11(18.6)$

$49(83.1)$

$10(16.9)$

$6(10.2)$

$0(0)$

Outcomes

Total ICU length of stay (hours, median, IQR)

Total hospital length of stay (days, median, IQR)

$47(29-71)$

Patients requiring post-operative packed red blood cells (\%)

Atrial fibrillation (\%)

$8(6-9)$

$25(42.4)$

$9(15.3)$

Cerebrovascular accident (\%)

Re-operation for bleeding (\%)

Acute kidney injury requiring hemodialysis (\%)

Q wave myocardial infarction (\%)

30-day mortality (\%)

Clinical follow-up available (\%)

Time to last follow-up (months, mean \pm SD)

Acute coronary syndrome (\%)

Target vessel revascularization (\%)

Cerebrovascular accident (\%)

$2(3.4)$

$3(5.1)$

$3(5.1)$

$0(0)$

2 (3.4)

59 (100)

$15 \pm 8$

$5(8.5)$

$0(0)$

$0(0)$

Hospitalization due to CHF, ACS, or bleeding (\%)

All-cause mortality at follow-up (\%) coronary artery bypass graft and valve surgery. We evaluated the outcomes of this hybrid approach. Methods: We retrospectively evaluated the outcomes of consecutive patients with significant proximal LAD coronary artery and valvular heart disease who underwent PCI followed by elective MIVS at our institution between February 2009 and July 2013. A Kaplan-Meier analysis was performed to estimate mid-term survival. Results: A total of 59 patients were identified, with a mean age of $75.3 \pm 8.8$ years (table 1 ). PCI was performed for 1- or 2-vessel disease in $76.3 \%$ and $23.7 \%$ of the patients, respectively. Drug-eluting stents were used in $76.3 \%$ of the patients, and $76.3 \%$ were on dual anti-platelet therapy at the time of MIVS. Within a median of 39 days (IQR 10-57), 90\% patients underwent primary and $10 \%$ underwent re-operative MIVS. Post-operatively, there were $2(3.4 \%)$ cerebrovascular accidents, and $3(5.1 \%)$ patients requiered re-operation due to bleeding. The operative mortality was $3.4 \%$. The 30 -day outcomes of patients on dual antiplatelet therapy at the time of MIVS were not significantly different from those not on it, with the exception of the number of patients requiring postoperative red blood cell tranfusion (51 vs. $14 \%, \mathrm{p}=0.01$ ). Follow-up was available for all the patients. At a mean follow-up period of $15 \pm 8$ months, $8.5 \%$ of the patients had a non-target vessel acute coronary syndrome and the survival rate was $86.4 \%$. Conclusions: In a select group of patients with proximal LAD coronary artery and valvular disease, a hybrid approach of PCI followed by MIVS can be safely performed with excellent mid-term outcomes.

\section{Does the Number of Redo Mitral Valve Replacement for Structural Valve Deterioration Affect the Early and Late Outcomes?}

\author{
Naoto Fukunaga, Kenta Nishiya, Yoshito Sakon, \\ Yasunobu Konishi, Ken Nakamura, Yoshiaki Saji, \\ Hideo Kanemitsu, Tadaaki Koyama
}

Kobe City Medical Center General Hospital, Kobe, Japan

Objective: We analyzed whether number of redo mitral valve replacement (MVR) for structural valve deterioration (SVD) affects the early and late survival. Methods: We retrospectively analyzed consecutive 114 patients who underwent redo MVR for SVD from January 1990 to December 2010. Mean age was $63.4 \pm 11.9$ years. The 27 patients $(23.7 \%$ ) underwent redo heart valve operation more than first redo. New York Heart Association (NYHA) functional class III or IV was present in 51 (44.7\%). Eighty-four patients $(74.7 \%)$ had preoperative atrial fibrillation. Mean follow-up was $8.5 \pm 6.8$ years. Results: Mean operative interval was $11.7 \pm 3.8$ years. In current redo operation, biological valve was implanted in 56 patients and mechanical in 58. Overall hospital mortality was $8.8 \%$. Hospital mortality of first redo cases was $4.6 \%$, while that of second and more cases $22.2 \%(p=0.5212)$. Logistic regression analysis identified number of redo operation ( $\mathrm{OR}=4.399 ; p=0.0467)$ and NYHA functional class III or IV $(\mathrm{OR}=9.090 ; p=0.0483)$ as an independent risk factor for hospital mortality. Long-term survival at 5, 10 and 15 years were $86.5 \% \pm 3.6 \%, 72.6 \% \pm 5.5 \%$ and $60.7 \% \pm 6.7 \%$, respectively. There were 26 late deaths. On Cox regression analysis, advanced age was an only predictor of late mortality $(\mathrm{HR}=1.098$; 95\% CI, 1.037 $1.163 ; p=0.0013$ ). There was no significant difference of $5-, 10$ - and 
15-year survivals for the first redo and second and more group $(88.2 \% \pm 3.9 \%$ versus $79.0 \% \pm 10.8 \%, 70.8 \% \pm 6.4 \%$ versus $70.2 \% \pm 12.7 \%$ and $57.5 \% \pm 8.0 \%$ versus $70.2 \% \pm 12.7 \%$, respectively, log-rank $p=$ 0.3369 ). However, there was statistically significant difference of late survival between patients with redo biological and mechanical valve replacement $(\log$-rank $p=0.0002)$. Conclusions: Early mortality of redo operation more than first is much higher than that in first redo. However, it might be considered to perform the second or more redo operations carefully because late survival of MVR for SVD was similar between first and second or more redo operations.

\section{Retrospective Analysis of 1476 Cases of Heart Valve Replacement in Xinjiang Area}

\section{Abudunaibi Maimaitiaili, Mulati Abudureheman, correspond, Aisikaer Shabiti, Huo Qiang}

First Affiliated Hospital of Xinjiang Medical University, Urumqi, China

Objective: To explore heart valve replacement perioperative experience in Xinjiang area, China. Methods: A total of 1476 cases were carried out valve replacement in normathermia under cardiopulmonary bypass surgery From 1993.12 to 2012.12 in our institute. And the clinical characteristic, indications and treatments of the patients were analyzed retrospectively. Results: Among 1476 cases the overall recovery were 1421 cases (96.3\%) and 55 (3.7\%) patients died of postoperative complications. The mortality had significant differences between different age groups and different NYHA classification groups; There was no significant difference between different operation time groups and different cardiothoracic ratio groups. Conclusion: Valve replacement surgery is still an effective means in the treatment of valvular disease in Xinjiang area. Enhancing preoperative care for elders and patients with poor cardiac function can decrease the operative complication and mortality rate so as to improve the treatment effect.

\section{Mechanism of Left Ventricular Hyperkinetic Motion in Patients with Mitral Regurgitation: A Study Using Two-Dimensional Speckle Tracking Analysis}

\author{
Kazato Ito ${ }^{1}$, Yukio Abe ${ }^{2}$, Chiharu Tanaka ${ }^{2}$, Atsuko Furukawa ${ }^{2}$, \\ Kentaro Yano ${ }^{1}$, Daisuke Tonomura' , Kosuke Takehara', \\ Naoto Kino ${ }^{1}$, Keiichi Furubayashi ${ }^{1}$, Yoshihisa Shimada ${ }^{1}$, \\ Toshiya Kurotobi ${ }^{1}$, Takao Tsuchida ${ }^{1}$, Hitoshi Fukumoto', \\ Minoru Yoshiyama ${ }^{3}$, Junichi Yoshikawa ${ }^{4}$, Takahiko Naruko², \\ Toshihiko Shibata ${ }^{2}$ \\ ${ }^{1}$ Shiroyama Hospital, Habikino, Japan; ${ }^{2}$ Osaka City General \\ Hospital, Osaka, Japan; ${ }^{3}$ Osaka City University Medical School, \\ Osaka, Japan; ${ }^{4}$ Nishinomiya Watanabe Cardiovascular Center, \\ Nishinomiya, Japan
}

Background: Mitral regurgitation (MR) is a valvular disease characterized by compensative volume overload and left ventricular (LV) hyperkinetic motion. However, the details of LV hyperki- netic motion in MR patients are still unknown. The purpose of this study was to investigate the mechanism of LV hyperkinetic motion in patients with MR using speckle tracking analysis in two-dimensional echocardiography. Methods: Twelve patients with severe primary MR and LV ejection fraction above 60\% underwent echocardiography. Patients with coronary artery disease, cardiomyopathy, or atrial fibrillation were excluded. Global LV radial strain, global LV endocardial longitudinal strain, global LV epicardial longitudinal strain, LV sphericity index at end-diastole, and LV sphericity index at end-systole were derived from two-dimensional speckle tracking analysis in the four-chamber view. The results were compared with those in 12 healthy controls. Results: There was no significant difference in LV ejection fraction between MR patients and controls ( $70 \pm 7 \%$ vs. $66 \pm 6 \%)$. However, LV end-diastolic volume and LV total stroke volume were significantly larger in MR patients than in controls $(121 \pm 31 \mathrm{ml}$ vs. $79 \pm 22 \mathrm{ml}, \mathrm{p}=$ 0.0008 ; and $85 \pm 26 \mathrm{ml}$ vs. $51 \pm 13 \mathrm{ml}, \mathrm{p}=0.0006$, respectively). There was no significant difference in global LV endocardial longitudinal strain or epicardial longitudinal strain between MR patients and controls $(-20 \pm 5 \%$ vs. $-21 \pm 5 \%$; and $-9 \pm 3 \%$ vs. $-11 \pm 3 \%$, respectively). In contrast, global LV radial strain was significantly lager in MR patients than in controls $(37 \pm 12 \%$ vs. $25 \pm 9 \%, \mathrm{p}=0.0096)$. LV sphericity index decreased from $0.71 \pm 0.13$ at end-diastole to $0.58 \pm 0.12$ at end-systole in MR patients, and the change was significantly larger in MR patients than in controls $(-0.13 \pm 0.08$ vs. $-0.05 \pm 0.07, \mathrm{p}=0.016)$. Conclusions: LV hyperkinetic motion in patients with severe MR does not depend on the increase in LV myocardial shortening in a longitudinal direction, but depends on the increase in LV myocardial thickening and inward motion in a transverse direction.

\section{Vascular Landmarks on the Ross Operation. The Rule of the Right Vessels and Av Block}

\section{Gustavo Abuin}

Fernandez Hospital, buenos aires, Argentina

Objective: The role of the firs septal artery is well known in the blood supply of the zone of the extraction of the autograft ('Ross zone'). However, the right superor artery is not considered as an impoortant source of supply of the 'Ross zone'. Methods: There were dissected 20 hearts injectded with latex in the coronary arteries. The whole heart conducition system was dissected and its blood supply. It was dissected also the blood supply of the 'Ross zone'. Magnification with a surgical microscope was used throughout the procedure. Results: The blood supply of the zone of extraction of the autograft (Ross zone) was clearly demonstrated and the rule of the supply of these vessels in the nourishment of the heart conduction system. Conclusions: The role of the right coronary artery is well demonstrated in several anatomical specimens. This new point and clear point of view of the anatomy of the blood supply of the 'ross zone' and the heart conduction system must be take into account in order to perform an autograft operation. 


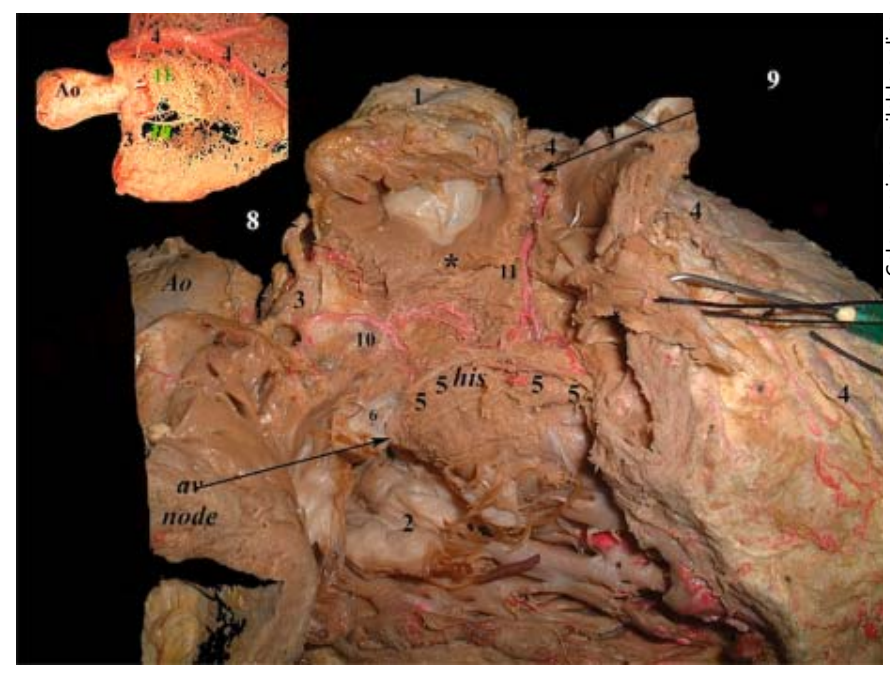

Fig. 1. (for Abstract of Gustavo Abuin).

\section{Clinical Outcomes and Echocardiological Hemodynamics of the Aortic Valve Reconstruction using Autologous Pericardial Leaflet - Comparison With the Bioprosthesis Valves}

\author{
Keisuke Watadani, Taijiro Sueda, Katsuhiko Imai, \\ Tatsuya Kurosaki, Taiichi Takasaki, Sinya Takahashi, \\ Keijiro Katayama, Takahiro Tguchi
}

Hiroshima University of Medicine, Hiroshima, Japan

Objective: In Aortic valve replacement, small size valve have a problem of higher postoperative pressure gradient and lower rehabilitation. Compared to Anglo-Saxon, Japanese patients were used smaller size valve. Bioprosthesis valve implantation is the preferred choice for AVR in the elderly as a result of the shorter life expectancy and reduced need of anticoagulation. However, despite the progress of the design and construction of prosthetic valves, hemodynamic performance is not yet comparable to that of native aortic valve. We performed aortic vale reconstruction ( $\mathrm{AVrC})$ using glutaraldehyde-treated autologous pericardium with Ozaki's developed sizing apparatus and template. This is the first time to report the clinical outcomes and echocardiological hemodynamics of $\mathrm{AVrC}$ compared with the bioprosthesis valve. Methods: The study group consisted of 79 patients with aortic stenosis who underwent elective AVR with a bioprosthesis or autologous pericardial at Hiroshima University Hospital between January 2008 and May 2013. Of them 47 were treated with a CEP bioprosthesis (Edwards Lifescience, Irvine, CA, USA) and 10 with an autologous pericardial leaflet. Results: In hospital death, all over survival rate and cardiac event free rate were not significant change in $\mathrm{AVrC}$ compared with bioprosthesis valve. Aortic valve peak flow and LVaortic mean pressure gradient at after 6 months were significantly lower in AVR group ( $\mathrm{p} \leq 0.01)$. In AVrC group, aortic valve annular size at discharge was not significant change surging 1 cycle. But at after 6 months and 1 year, annular size changing rate was
Table 1. Echocardiography at pre and postoperative (for Abstract of Keisuke Watadani)

\begin{tabular}{lccr}
\hline $\begin{array}{l}\text { Max Ao flow } \\
(\mathrm{m} / \mathrm{s})\end{array}$ & $\mathrm{AVrC}(\mathrm{n}=10)$ & $\begin{array}{l}\text { Bioprosthesis } \\
(\mathrm{n}=47)\end{array}$ & $\mathrm{p}$ \\
\hline Preoperative & $5.16 \pm 0.69$ & $4.93 \pm 1.36$ & 0.260 \\
At discharge & $2.90 \pm 0.74$ & $2.57 \pm 0.49$ & 0.034 \\
After 6 months & $2.20 \pm 0.07$ & $2.60 \pm 0.66$ & $<0.001$ \\
After 1 year & $2.23 \pm 0.61$ & $2.68 \pm 0.43$ & 0.735 \\
\hline Mean LVAo-PG & & & \\
(mm Hg) & & & \\
\hline Preoperative & $63.21 \pm 16.08$ & $59.93 \pm 29.40$ & 0.271 \\
At discharge & $19.11 \pm 9.51$ & $14.93 \pm 5.92$ & 0.053 \\
After 6 months & $9.20 \pm 1.48$ & $16.10 \pm 6.91$ & 0.001 \\
After 1 year & $13.75 \pm 10.25$ & $15.71 \pm 5.44$ & 0.224 \\
\hline
\end{tabular}

significant larger compared with discharge $(\mathrm{p}<0.001)$. Conclusions: AVrC provides good short and mid term echocardiological hemodynamics compared with bioprosthesis valve. Assessment of long-term data will be examined in the future.

\section{Clinical and Echocardiographic Impact of Trifecta $^{\mathrm{TM}}$ Aortic Bioprosthesis: An Initial Single-Centre Experience}

Inna Kammerer, Mona Wiedemann, Falk-Udo Sack

Department of Cardiac Surgery, Ludwigshafen, Germany

Objectives: To face common issues such as high transvalvular pressure gradients after implanting small valve sizes and low effective orifice areas, which leads into manifestation of a patient prosthesis mismatch, the new Trifecta ${ }^{\mathrm{TM}}$ aortic valve was designed as a stented valve for supra-annular placement to re-duce the obstruction of the left ventricular outflow tract. The present study evaluated retrospective the early haemodynamic performance after implantation of the new valve. Methods: Between September 2010 and February 2013, 380 patients underwent an aortic valve replacement (AVR) with Trifecta ${ }^{\mathrm{TM}}$, Department of Cardiac Surgery, Germany. Prevalent cause of AVR was single aortic stenosis in $52 \%$, insufficiency in $10 \%$ and combined pathology in $38 \%$. Mean age at patients was 73 years (range 33-93), 58\% of them were male. The mean preoperatively pressure gradient was $45 \mathrm{~mm} \mathrm{Hg}$ with effective orifice area of $0.73 \mathrm{~cm}^{2}$. The majority of patients were classified as NYHA Class III (78\%). Results: The 30-day-mortality was $4 \%(\mathrm{n}=16)$. The early postoperative hemodynamic date showed in figure 1 . Concomitant procedures were most frequently coronary artery bypass grafting $(\mathrm{n}=234)$, ablation of atrial fibrillation with LAA exclusion $(n=67)$. There were 2 cardiac depending intraoperative deaths $(0.52 \%)$, but not valve related. At discharge, there could not be determined any valve thrombosis or prosthesis failure. Conclusion: The Trifecta ${ }^{\mathrm{TM}}$ reveals excellent early haemodynamics over all valve sizes. Especially low gradients 
Fig. 1. Echocardiographic haemodynamic data for each Trifecta ${ }^{\mathrm{TM}}$ aortic valve size $(\mathrm{n}=380)$ (for Abstract of Inna Kammerer).

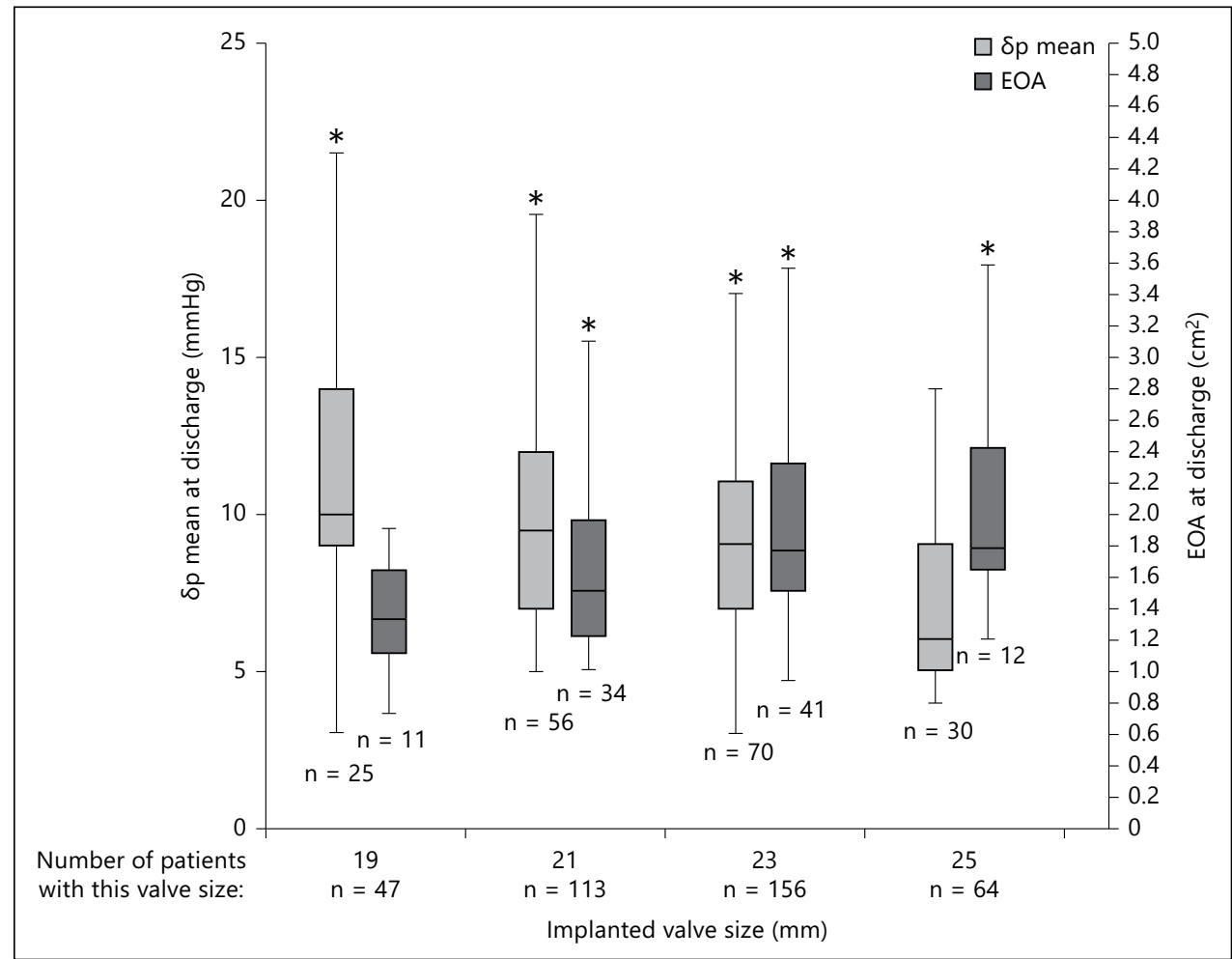

in small valves and large EOAs attest a satisfying outcome after implantation. Futher data is needed to investigate the stableness of these results in long time follow-up.

\section{Left Ventricular Remodelling After Mitral Valve Reconstruction: A Prospective cMRI Study}

Inna Kammerer ${ }^{1}$, Armin Vomend ${ }^{1}$, Bernd Cornelius ${ }^{2}$, Günter Layer ${ }^{2}$, Falk-Udo Sack

${ }^{1}$ Department of Cardiac Surgery, Ludwigshafen, Germany; ${ }^{2}$ Department of Radiology, Ludwigshafen, Germany

Objective: The aim of this prospective study is the evaluation of the postoperative remodelling of the left ventricle (LV) after mitral valve reconstruction (MVR) in patients with isolated mi- tral valve disease. Methods: Since 201149 patients were enrolled. In a prospective trial, LV geometry was assessed by the cardio magnetic resonance imaging (cMRI) pre-, early and 12 months postoperatively. Of these, $38(78 \%)$ were men, the mean age was $60+15$ years. Minimal invasive operation technique (MIC) was done in more than $85 \%$ of MVR. Preliminary Results: One patient died 4 months after MVR due to an aortic dissection Stanford $\mathrm{A}$. The 1 year freedom from reoperation or severe recurrent insufficiency was $92 \%(\mathrm{n}=44)$. The mean ischemia time observed $73+20 \mathrm{~min}$, the mean hospital duration was $10+4$ days. The posterior leaflet correction with mean $34+2 \mathrm{~mm}$ ring size was made in $81 \%$ of patients $(n=40)$, in $17 \%(n=8)$ only ring without leaflet surgery and in $2 \%(\mathrm{n}=1)$ anterior leaflet rectification. The 1 year follow-up was done in $79 \%(n=33)$. The end-systolic and -diastolic volumes, cor weight measurement in cMRI diagnostic observed incurred levels in hospital and 1 year follow-up history. Other remodelling parameters of cMRI showed no differences in methods (table 1). Conclusion: Measurements of ventricular ge-

Table 1.cMRI parameters in pre-, early and 12 months postoperative clinical date (EF = ejection fraction, EDD) (for Abstract of Inna Kammerer)

\begin{tabular}{llllllll}
\hline $\begin{array}{l}\mathrm{n}=49 \\
\text { mean }\end{array}$ & EF preOP & EF pod & $\begin{array}{l}\text { EF 12 months EDD preOP } \\
\text { pod }\end{array}$ & EDD pod & $\begin{array}{l}\text { EDD 12 months } \\
\text { pod }\end{array}$ & $\begin{array}{l}\text { EDV } \\
\text { preOP }\end{array}$ & $\begin{array}{l}\text { EDV pod } \\
\text { EDV 12 } \\
\text { months pod }\end{array}$ \\
\hline cMRI & $63 \%+12$ & $55 \%+12$ & $54 \%+13$ & $56 \mathrm{~mm}+7$ & $50 \mathrm{~mm}+6$ & $47 \mathrm{~mm}+9$ & $165 \mathrm{ml}+46126 \mathrm{ml}+31$ \\
\hline & $\begin{array}{l}\text { ESV } \\
\text { preOP }\end{array}$ & $\begin{array}{l}\text { ESV } \\
\text { pod }\end{array}$ & $\begin{array}{l}\text { ESV 12 } \\
\text { months pod }\end{array}$ & $\begin{array}{l}\text { Cor weight } \\
\text { preOP }\end{array}$ & $\begin{array}{l}\text { Cor weight } \\
\text { pod }\end{array}$ & $\begin{array}{l}\text { Cor weight } \\
12 \text { months pod }\end{array}$ & \\
\hline cMRI & $64 \mathrm{ml}+26$ & $57 \mathrm{ml}+21$ & $41 \mathrm{ml}+14$ & $150 \mathrm{mg}+34$ & $148 \mathrm{mg}+34$ & $113 \mathrm{mg}+27$ & \\
\hline
\end{tabular}


ometry with the cMRI proved to be important imaging tools in assessing reverse LV remodelling. There is a clear tendency for postoperative remodelling. Long term follow-up (1 year) in a biger population will provide more information of the dynamics of this process.

\section{Left Ventricular Remodelling After Mitral Valve Reconstruction: A Prospective 3D TEE Study}

\section{Inna Kammerer, Armin Vomend, Falk-Udo Sack}

Department of Cardiac Surgery, Ludwigshafen, Germany

Objective: The aim of this prospective study is the evaluation of the postoperative remodelling of the left ventricle (LV) in after mitral valve reconstruction (MVR) in patients with isolated mitral valve disease. Methods: Since 201149 patients were enrolled. In a prospective trial, LV geometry was assessed by the real-time 3-dimensional transoesophageal echocardiography (3D TEE) pre-, early and 12 months postoperatively. Of these, 38 $(78 \%)$ were men, the mean age was $60+15$ years. Minimal invasive operation technique (MIC) was done in more than $85 \%$ of MVR. Preliminary Results: One patient died 4 months after MVR due to an aortic dissection Stanford A. The 1 year freedom from reoperation or severe recurrent insufficiency was $92 \%$ ( $n=$ 44). The mean ischemia time observed $73+20 \mathrm{~min}$, the mean hospital duration was $10+4$ days. The posterior leaflet correction with mean $34+2 \mathrm{~mm}$ ring size was made in $81 \%$ of patients $(\mathrm{n}=40)$, in $17 \%(\mathrm{n}=8)$ only ring without leaflet surgery and in $2 \%(\mathrm{n}=1)$ anterior leaflet rectification. The 1 year follow-up was done in $79 \%(n=33)$. The $L V$ remodelling parameters in 3D TEE diagnostic observed incurred levels of pres-sures in hospital and 1 year follow-up history (table 1). Conclusion: Measurements of ventricular geometry with the $3 \mathrm{D}$ transoesophageal echocardiography TEE proved to be important imaging tools in assessing reverse LV remodelling. There is a clear tendency for postoperative remodelling. Long term follow-up (1 year) in a biger population will provide more information of the dynamics of this process.

\section{Impact of Mitral Regurgitation's Etiology on Perioperative Outcomes in Mitral Valve Surgery}

Fabio Barili, Alberto Battisti, Francesco Rosato, Claudio Grossi

S.Croce Hospital, Cuneo, Italy

Objective: The identification of mitral regurgitation etiology represents a central issue in the planning of surgical options and in risk stratification. Several studies has analyzed outcomes of mitral valve repair in specific etiologic subgroups, without evaluating the direct impact of MR etiology adjusted for confounding factors. This study was undertaken to evaluate the role of mitral regurgitation's etiology on perioperative mortality and intensivecare-unit length of stay (ICU-LOS) in patients who underwent isolated or associated mitral surgery. Methods: Data on 950 consecutive patients who underwent isolated or associated surgery for mitral regurgitation were prospectively collected in a 7-year period. Potential risk factors for perioperative mortality and ICULOS were analyzed through logistic regression and accelerated failure time parametric models respectively. Results: The etiology of mitral regurgitation was ischemic (IMR) in 386 patients, functional non-ischemic (FNIMR) in 114 and organic in 450. Perioperative mortality was $3.6 \%$ (34 pts) and median ICU-LOS was 1 day. Although IMR and FNIMR were found associated to death at preliminary analysis (OR 5.6 and 4.9, respectively), the subsequent multivariate analysis demonstrated that independent predictors were only age (OR 5.5 at 80 years), previous cardiac surgery (OR 4.0), ejection fraction lower than $30 \%$ (OR 11.3), EF between 30 and 50\% (OR 4.1), the emergency status (OR 17.7) and ECC time (OR 8.5 at three-hour ECC). Several perioperative factors were found related to ICU-LOS prolongation and the strongest effect was observed for EF lower than 30\% (OR 13.3; 95\% CI 8.9-20.0), IABP use (OR 6.2; 95\% CI 2.8-13.9). Differently from risk model for mortality, IRM but not FNIRM was an independent predictor of prolongation of ICU-LOS (OR 1.5, 95\% CI: 1.12.1). Conclusions: The etiology of mitral regurgitation does not affect in-hospital mortality. Nonetheless, IMR is an independent risk factor for prolonged ICU-LOS and hence for complicated postoperative clinical course.

Table 1.3D TEE parameters in pre-, early and 12 months postoperative clinical date (EF = ejection fr) (for Abstract of Inna Kammerer)

\begin{tabular}{|c|c|c|c|c|c|c|c|c|c|}
\hline $\begin{array}{l}\mathrm{n}=49 \\
\text { mean }\end{array}$ & $\begin{array}{l}\mathrm{EF} \\
\text { preOP }\end{array}$ & $\begin{array}{l}\text { EF } \\
\text { pod }\end{array}$ & $\begin{array}{l}\text { EF } 12 \text { months } \\
\text { pod }\end{array}$ & EDP preOP & $\begin{array}{l}\text { EDP } \\
\text { pod }\end{array}$ & $\begin{array}{l}\text { EDP } \\
12 \text { months pod }\end{array}$ & ESP preOP & ESP pod & $\begin{array}{l}\text { ESP } \\
12 \text { months pod }\end{array}$ \\
\hline \multirow[t]{2}{*}{ 3D TEE } & $59 \%+9$ & $57 \%+10$ & $51 \%+10$ & $\begin{array}{l}52 \mathrm{~mm} \mathrm{Hg}+ \\
9\end{array}$ & $53 \mathrm{~mm} \mathrm{Hg}+7$ & $54 \mathrm{~mm} \mathrm{Hg}+7$ & $36 \mathrm{~mm} \mathrm{Hg}+8$ & $38 \mathrm{~mm} \mathrm{Hg}+8$ & $37 \mathrm{~mm} \mathrm{Hg}+6$ \\
\hline & LVOT preOP & LVOT pod & $\begin{array}{l}\text { LVOT } \\
12 \text { months pod }\end{array}$ & LA preOP & LA pod & $\begin{array}{l}\text { LA } 12 \text { months } \\
\text { pod }\end{array}$ & & & \\
\hline 3D TEE & $\begin{array}{l}0.86 \mathrm{~m} / \mathrm{sec}+ \\
0.32\end{array}$ & $\begin{array}{l}1.06 \mathrm{~m} / \mathrm{sec}+ \\
0.39\end{array}$ & $\begin{array}{l}0.81 \mathrm{~m} / \mathrm{sec}+ \\
0.17\end{array}$ & $46 \mathrm{~mm}+9$ & $45 \mathrm{~mm}+7$ & $46 \mathrm{~mm}+8$ & & & \\
\hline
\end{tabular}




\section{Coronary Ostial Compromise in Aortic Valve Replacement: A Rare But Avoidable Fatal Complication}

Shakil Farid, Aravinda Page, Neil Howell, Yasir Abu-Omar, Martin Goddard, David Jenkins, Samer Nashef

Papworth Hospital, Papworth, United Kingdom

Objective: To determine the incidence and mechanism of surgical compromise of the coronary ostia in aortic valve replacement (AVR). Methods: Retrospective review of prospectively collected clinical data and autopsy findings in 322 patients who died in hospital after AVR with or without concomitant procedures in a single institution from January 1998 to March 2013. Results: Over the 15-year period, more than 17 surgeons performed 7507 AVRs with or without other procedures. The mean age was $70.8( \pm 11.78)$ years and $63 \%$ were male. Bioprosthetic valves were used in $75 \%$ of cases, mechanical valves in $24.7 \%$ and homografts in only $0.3 \%$. Early mortality for all patients (including combined, emergency and redo procedures) was $4.28 \%$ (mean logistic EuroSCORE 10.7). There were 322 deaths after procedures involving the aortic valve. Autopsy examination was carried out in all patients and showed that $3.4 \%(n=11)$ of deaths were at least in part attributed to encroachment on one or both of the coronary ostia. Causes of ostial compromise included the valve sutures, the valve sewing ring and the aortotomy suture line. Conclusion: Coronary ostial compromise in AVR is a very rare but real problem occurring in at least $0.14 \%$ of AVRs and contributing to or directly causing one in every 29 AVR deaths. Surgeons should have a high level of awareness of the risk of this rare but fatal and avoidable complication.

\section{Epidemiology and Clinical Profile of Severe Rheumatic Heart Disease in Malaysia}

\author{
Benjamin Levin ${ }^{1}$, Maizatu Akma Solong ${ }^{2}$, Norfazlina Jaffar ${ }^{2}$, \\ Siti Nurzaliana Mohd Safari ${ }^{2}$, M.F. Ramli ${ }^{2}$, M.A. Rosli ${ }^{2}$ \\ ${ }^{1}$ Duke University, Durham, NC, USA; ${ }^{2}$ National Heart Institute, \\ Kuala Lumpur, Malaysia
}

Objective: Rheumatic heart disease (RHD) is the most common cause of valvular heart disease in the developing world and a major global public health challenge; yet there is a paucity of data on severe RHD in Southeast Asia. We defined the epidemiology and clinical profile of severe RHD (defined as requiring surgical or procedural intervention) that presented to the largest academic cardiology hospital in Malaysia from 2010 through 2012. Methods: All patients with clinically severe RHD who underwent either PTMC or surgery from 1/2010 through $12 / 2012$ were identified from surgical OR and cath lab procedure lists. All of the patients' medical records were reviewed. Results: A total of 552 patients were identified ( 480 adult, 72 pediatric). 403 adult (66\% female, $34 \%$ male) and 70 pediatric ( $50 \%$ male, $50 \%$ female) patients underwent surgical valve repair or replacement. Of the 403 adult surgical patients, none of them had either a reported history of Group A streptococcal (strep) pharyngitis, primary or secondary antibiotic prophylaxis. $6 \%$ reported history of acute rheumatic fever
(ARF). Surgery involved 1 (37\%), 2 (50\%) or 3 heart valves (13\%). $90 \%$ of adult patients had mitral valve surgery (16\% mitral valve repair), $65 \%$ aortic valve surgery, $47 \%$ tricuspid valve surgery. Of the 70 surgical pediatric patients, none of them had a reported history of group A strep pharyngitis or primary antibiotic prophylaxis. $17 \%$ had history of history of ARF and $28 \%$ history/active carditis. Surgery involved 1 (40\%), 2 (23\%) or 3 heart valves (7\%). $91 \%$ of pediatric patients had mitral valve surgery $(70 \%$ mitral valve repair), $31 \%$ aortic valve surgery, $30 \%$ tricuspid valve surgery. Conclusions: There was a significant prevalence of severe rheumatic heart disease during the three year period of 2010-2012. Patients did not report any known history of prior group A strep pharyngitis or primary antibiotic prophylaxis and low known incidence of ARF. There was significant aortic valve involvement and a predominance of female adult surgery patients. There is a strong need for better public health education and prevention of RHD in Malaysia.

\section{Mid-Term Echocardiographic Follow-Up of Mitral Valve Durability Following Robotic Mitral Repair}

\author{
Jae Suk Yoo, Joon Bum Kim, Sung-Ho Jung, Suk Jung Choo, \\ Cheol Hyun Chung, Jae Won Lee
}

Asan Medical Center, University of Ulsan College of Medicine, Seoul, Republic of Korea

Objective: To review a single surgeon's experience on robotic mitral valve (MV) repair for mitral regurgitation (MR), focusing on the mid-term echocardiographic mitral durability. Methods: A total of 200 patients who underwent robotic MR repair using da Vinci system between August 2007 and December 2012 were evaluated. Transesophageal echocardiography was used intraoperatively to estimate the results and regular transthoracic echocardiographic follow-up was performed to detect MR recurrence. Results: MR repair was successfully performed with none or mild residual MR in $98.0 \%$ (figure 1a), without any conversion to sternotomy. Various repair techniques including ring annuloplasty (99.5\%), artificial chordae formation (45.5\%), leaflet resection (44.5\%), commissuroplasty (35.0\%), sliding annuloplasty (3.0\%), chordal transfer $(2.5 \%)$, cleft repair $(2.5 \%)$, papillary muscle release $(1.5 \%)$ and leaflet augmentation (1.1\%) were utilized. Concomitant maze procedures were performed in 44 patients $(22.0 \%)$. There were no in-hospital deaths. Clinical follow-up was complete in $96.5 \%$ of patients with a median of 31.4 months (inter-quartile range: $12.4-42.3$ months), during which 4 late deaths, 2 strokes, 1 low cardiac output, 1 newly required dialysis, and 1 reoperation for MR occurred. Freedom from major adverse cardiac and cerebrovascular events at 5 years was $87.7 \pm 5.1 \%$. Regular echocardiographic follow-up (more than 6 months) was possible in 187 patients $(93.5 \%)$. At a median of 29.6 months (inter-quartile range: 14.9-45.8 months), 21 (10.5\%) experienced moderate or greater MR. Freedom from moderate or greater MR at 5 years was $87.0 \pm 2.6 \%$ (figure $1 \mathrm{~b}$ ). Mean cardiopulmonary bypass and crossclamping times were $182.9 \pm 48.4$ and $110.9 \pm 34$. 1 minutes, respectively, both of which were significantly decreased based on chronological date of operation. Conclusions: Robotic MV repair is 


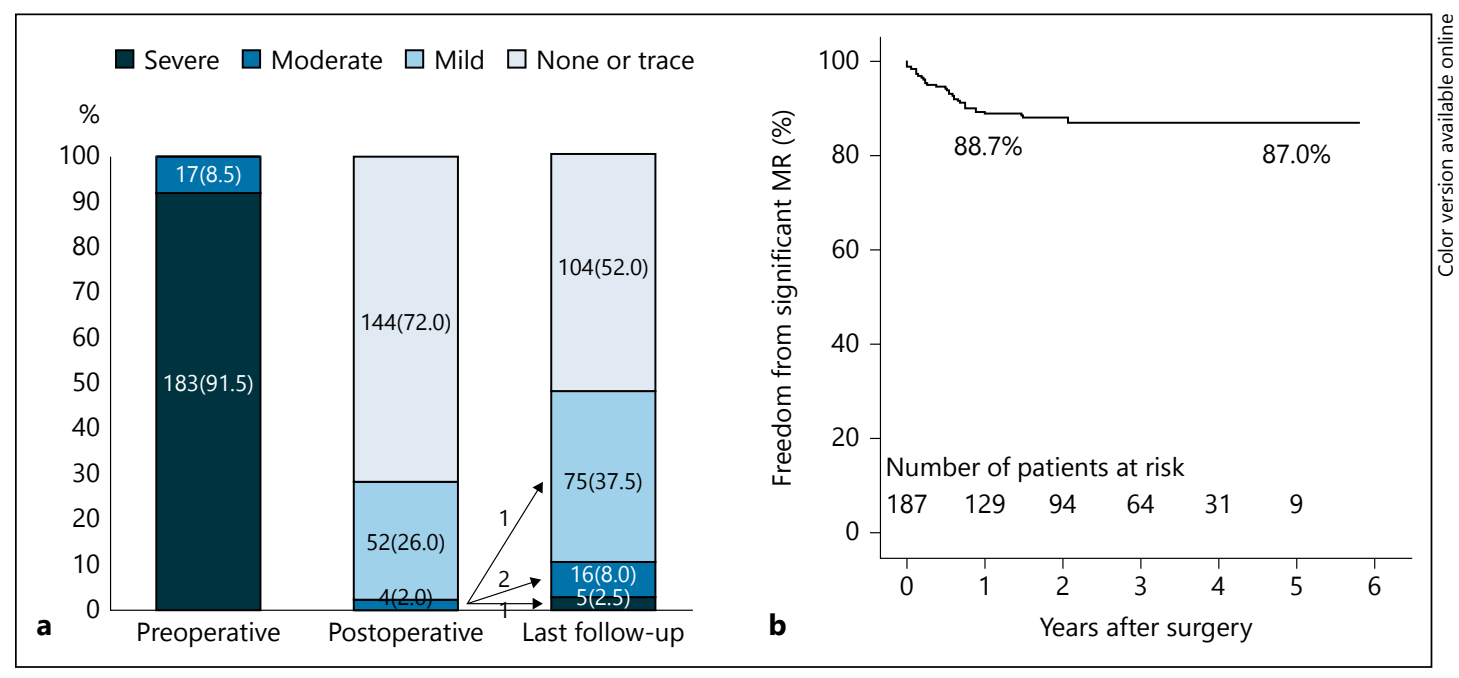

Fig. 1. (for Abstract of Jae Suk Yoo).

technically feasible and efficacious in terms of favorable mid-term mitral durability, utilizing various types of repair techniques with reasonable outcomes, and with improvement in procedure times as experience is accumulated.

\section{Aortic Valve Interstitial Cell Activation in Patients with Continuous-Flow Left Ventricular Assist Device Support}

Jan Willem van Rijswijk ${ }^{1}$, Jerson Martina ${ }^{2}$, Nicolaas de Jonge ${ }^{3}$, Roel A. de Weger ${ }^{1}$, Aryan Vink ${ }^{1}$, Jolanda Kluin ${ }^{2}$

${ }^{1}$ Pathology, University Medical Center, Utrecht, Netherlands; ${ }^{2}$ Cardiothoracic Surgery, University Medical Center, Utrecht, Netherlands; ${ }^{3}$ Cardiology, University Medical Center, Utrecht, Netherlands

Objective: Phenotypic changes of valvular interstitial cells (VICs) play a key role in valve tissue remodeling and pathology. In patients with continuous-flow left ventricular assist devices (cfLVADs), aortic valve commissural fusion and de novo aortic regurgitation have been reported. The underlying mechanisms of these phenomena are unknown. The aim of this study was to elucidate LVAD-induced changes in morphological structure and cellular behavior of the aortic valve. Methods: Aortic valves from hearts of 16 patients ( 11 male; mean age 52 year [26-68 years]) supported by cf-LVAD for an average duration of 754 days [71922 days] were examined after heart transplantation $(n=11)$ or autopsy $(\mathrm{n}=5)$. Histology was assessed by staining with hematoxylin and eosin, Verhoeff-van Gieson, and Sirius red (SR). Immunohistochemistry was performed on markers for valve interstitial cell (VIC) activation (alpha-smooth muscle actin ( $\alpha$ SMA)) and collagen synthesis (heat shock protein-47 (Hsp47)). Sections were graded semi-quantitatively and compared to patient clinical data. Results: De novo mild aortic regurgitation was observed in $77 \%$ of
cf-LVAD patients. Fusion ( $>3 \mathrm{~mm}$ ) was observed in $69 \%(11 / 16)$ aortic valves. Fusion areas consist of loosely arranged, non-inflammatory, fibrous tissue at the coapting surface. VIC activation in the aortic valve leaflet, as indicated by aSMA expression, positively correlated to the duration of cf-LVAD support ( $\mathrm{r} 2=0.51 ; \mathrm{p}<0.01)$, and with continuous aortic valve closure $(\mathrm{r} 2=0.61 ; \mathrm{p}<0.01)$. Collagen deposition and synthesis did not correlate to LVAD-support as indicated by SR staining and Hsp47 expression. Sclerotic change in the aortic root (mostly consisting of foam cells, cholesterol clefts and calcification) and aortic root diameter positively correlated to age $(\mathrm{r} 2=0.48 ; \mathrm{p}<0.001$ and $\mathrm{r} 2=0.54 ; \mathrm{p}<0.001$, respectively) but not to the duration of support ( $\mathrm{p}=0.9$ and $\mathrm{p}=0.7$, respectively). Conclusions: Duration of continuous flow support on LVAD and continuous aortic valve closure are associated with progressive VIC activation in aortic valve leaflets, eventually leading to leaflet fusion and/or regurgitation. These results suggest that short periods of valve opening may preserve a quiescent VIC phenotype and valve functionality, which is desirable especially in patients bridged to recovery.

\section{Patient Specific Segmentation and Modelling of the Ischaemic Mitral Valve from 3D Echocardiography}

\section{Abdullrazak Hossien}

Morriston University Hospital, Swansea, United Kingdom

Purpose: The surgical repair of ischemic mitral regurgitation (IMR) is very complex procedure. We propose a new method to segment the ischemic mitral valve (MV) computationally to enhance the understanding of 3D complexity of MV and to create a mathematical model for further finite element simulation of MV. Method and Materials: We obtained images of the mitral valve using 3 dimensional transoephageal-echocardiography (3D TOE) with full measurements. 3D TOE image volumes of the mitral 
valve at mid-systole and mid-diastole were selected. The images were sliced and segmented computationally by using open source software program and mathematical equations to build a MV model. Results: A high quality image set of the mitral valve was retrieved from $3 \mathrm{D}$ TOE. The slicing of images results in 85 images of $0.35 \mathrm{~mm}$ thickness. The reconstruction of the slices results in $3 \mathrm{D}$ anatomical segmentation of the MV. Binary STL file (STereoLithography) was built by processing the segmented MV model which was meshed as well. Conclusions: Computational segmentation and modelling of ischemic mitral valve may improve the understanding of 3D complexity of MV and may facilitate diagnostic precision of IMR for further surgical planning.

\section{Is the Minimally Invasive Mitral Valve Repair with Artificial Chords Reproducible and Applicable in Routine Surgery?}

\section{Aristotelis Panos}

LA Tour Hospital, Geneva, Switzerland

Background: Traditionally a variety of resectional techniques and chordae transfer are used for the repair of tha prolapsed segments. These techniques are difficult to apply in video-assisted mitral surgery. The application of artificial chordae in the setting of the video assisted mitral valve surgery allow for efficient repair. The purpose of this study was to demostrate the effectiveness and reproducibility of this method in the setting of routine surgery and assess the stability of the " figure of eight-without pledjet» implantation of the neochords in this setting. Methods: From February 2008 to October 2013, 424 consecutive patients were operated under video assisted mitral valve repair for degenerative disease or healed endocarditis of the posterior, anterior or both leaflets. Were excluded the patients with stenotic mitral valve disease, active endocarditis, peripheral artery disease or previous right thorax surgery. Mean age was $55 \pm 18$ years, LVEF of $60 \pm 8 \%$ and mean NYHA class II (87.5\%). We have used in all patients neochordae and in 47 of them an association of leaflet resection and artificial chordae. Results: Mean clamping time was of $106 \pm 28 \mathrm{~min}$ and cardiopulmonary bypass time of $133 \pm 30 \mathrm{~min}$. A very low incidence of residual leakage, grade I/IV (3.5\%) were discovered postoperatively. Fifteen patients required multiple mitral repair attempts and had significantly longer cardiopulmonary bypass times with good final result. For $25 \%$ of the patients were fast track procedures with extubation on the operating table and the remain in the six postoperative hours. Hospital stay was $5 \pm 1.3$ days. No patient required reoperation. Five patients $(1.2 \%)$ required re-exploration for bleeding. One patient had a permanent cerebral attack. The 30-day mortality was of $2.7 \%$. Follow-up ranged from 3-60 months (transthoracic echocardiography at one month, 6 months and yearly after the operation) during which all of the patients remained with none or trace mitral valve regurgitation. No desinsertion or rupture of any chord loops was noticed. Conclusions: High rate of repair, reproducible results in the daily surgery and stable repair over the time are in favor of the use of the artificial chordae. Associated to the minimally invasiveness of the method is our preferred technique for mitral valve surgery.

\section{Single Interrupted Suturing for Redo Mitral Valve Replacement Provides an Appropriate Prosthesis Size and Comparable Early Clinical Outcomes}

\author{
Hiroshi Furukawa, Tekashi Honda, Noriaki Kuwada, \\ Takahiko Yamasawa, Kazuo Tanemoto
}

Kawasaki Medical School, Kurashiki, Japan

Background: The purpose of this study was to evaluate the early clinical outcomes and efficacy of single interrupted suturing for redo mitral valve replacement (MVR). Methods: Of 334 mitral valve surgery patients, 20 consecutive patients (12 women and 8 men, mean age $66 \pm 11$ years, ranging from 32 to 80 ) who underwent redo MVR in our institute between 2000 and 2013 participated in this study. The surgical indications for redo MVR were paravalvular leakage (PVL) in 10 patients, prosthetic valve endocarditis (PVE) in 5, mechanical valve thrombosis in 3, and structural bioprosthesis deterioration in 2 . The number of previous surgeries was one in 10 patients, two in 6 , and three in 4 . Emergent or urgent surgeries were performed in 5 patients. Sharp dissection was initially performed on one side of the previous prosthetic sewing-cuff and the overall sewing-cuff was thereafter completely removed following leaflet detachment. Results: The surgical procedure was successfully performed in all patients without any serious complications. Single interrupted suturing with a mean number of $32.7 \pm 3.0$ sutures, ranging from 28 to 40 , was performed to implant the new valve without exposing the rough surface of the mitral valve annulus, thereby allowing for the eventual implantation of larger prostheses. Bioprostheses were selected in 10 patients and mechanical valves in 10 patients. Fifteen patients $(75.0 \%)$ were able to receive the same- or larger-sized new prosthesis than that of previous surgeries. No patients exhibited postoperative residual PVL or PVE. The operative mortality (30-days) was $5.0 \%$, which was similar to that of MVR $(n=78): 2.6 \%(p=0.57)$. Conclusions: Single interrupted suturing for re-do MVR may allow for the implantation of larger prostheses and this novel maneuver may achieve acceptable early clinical outcomes.

\section{Aortic Valve Replacement in Heart Failure Patients: Full Sternotomy or Minimally Invasive Access?}

Sven Lehmann, Sergey Leontyev, Christian D. Etz, Anna Funkat, Jens Garbade, Martin Misfeld, Michael Borger, Friedrich W. Mohr

Heartcenter Leipzig, Leipzig, Germany

Objective: Minimally invasive techniques are progressively challenging traditional approaches in cardiothoracic surgery even for patients with a higher operative mortality. The aim of this study was to compare mortality and morbidity after minimally invasive (MIC) vs. conventional (CON) access for aortic valve replacement in patients with severely reduced ejection fraction (EF). Methods: 354 consecutive patients with severely reduced $\mathrm{EF} \leq 30 \%$ had undergone aortic valve replacement at our institution from 11/94 to 10/12: 39 patients had a MIC access and 315 a CON access. Results: There were no significant differences between the two 
groups (CON vs. MIV) in terms of mean age $(65 \pm 10$ vs. $67 \pm 13$ years), $\mathrm{EF}(24 \pm 5$ vs. $15 \pm 5 \%)$ and $\log$ Euroscore (17.7 \pm 17.2 vs. $15.0 \pm 9.3)$ MIC patients remained longer cross clamp time (53 \pm 17 vs. $63 \pm 16 \min \mathrm{p}=0.004)$ but are not longer on cardiopulmonary bypass ( $87 \pm 33$ vs. $92 \pm 29 \mathrm{~min}$ ) than CON patients. 30 day survival rate was $94.9 \pm 3.5(\mathrm{MIC})$ and $89.6 \pm 1.7(\mathrm{CON} ; \mathrm{p}=0.32)$. The Survival rate after 13 years was $23.2 \pm 9.3 \%$ (MIC) vs. $27.1 \pm 3.5 \%(\mathrm{CON}$; $\mathrm{p}=0.34)$. Univariate analysis indicated dialyses, atrial fibrillation, PM and ICD device, urgent or emergency operation, endocarditis and cardiogenic shock as risk factors for long term mortality. The multivariate analysis revealed urgent or emergency operation $(\mathrm{p}<$ 0.01 ) as independent risk factors for long-term mortality. Cox analysis revealed the following predictors for long term mortality: permanent haemodialysis ( $\mathrm{p}<0.01, \mathrm{OR}=5.0$ ), active endocarditis $(\mathrm{p}<0.01, \mathrm{OR}=6.1)$ and ICD wearer $(\mathrm{p}=0.04, \mathrm{OR}=9.3)$. Conclusions: In this heart failure cohort minimally invasive access for aortic valve replacement is at least as safe as the conventional approach. Emergency indication, permanent haemodialysis and active endocarditis are independent predictor for mortality. The operative trauma is clearly reduced. The main indication for full median sternotomy should be in emergency indications.

\section{Month Post-Market Registry Results in an All-Comers Population Implanted with The ACURATE TATM Transapical TAVI Device}

\author{
Jörg Kempfert ${ }^{1}$, Hendrik Treede ${ }^{2}$, David Holzhey ${ }^{3}$, \\ Steffen Hoffmann ${ }^{4}$, Evaldas Girdauskas ${ }^{5}$, Holger Schröfel ${ }^{6}$, \\ Matthias Thielmann ${ }^{7}$, Shahram Lofti ${ }^{8}$, Christophe Huber ${ }^{9}$, \\ Michael Hilker ${ }^{10}$, Nicolas Doll ${ }^{11}$, Klaus Matschke ${ }^{12}$, \\ Thorsten Wahlers ${ }^{13}$, Arno Diegeler ${ }^{14}$, Carlo Savini ${ }^{15}$, \\ Nalian Schnelle ${ }^{16}$, Carla Romina Agatiello ${ }^{17}$, \\ Hermann Reichenspurner ${ }^{2}$, Friedrich Mohr ${ }^{3}$, Thomas Walther ${ }^{1}$ \\ ${ }^{1}$ Kerckhoff-Klinikum, Bad Nauheim, Germany; ${ }^{2}$ University \\ Heart Center, Hamburg, Germany; ${ }^{3}$ Leipzig Herzzentrum, \\ Leipzig, Germany; ${ }^{4}$ Schüchtermann-Schillersche Klinik, \\ Bad Rothenfelde, Germany; ${ }^{5}$ Zentralklinik, Bad Berka, \\ Germany; ${ }^{6}$ Klinik für Herzchirurgie, Karlsruhe, Germany; \\ ${ }^{7}$ Universitätsklinikum, Essen, Germany; ${ }^{8}$ Universitätsklinikum, \\ Aachen, Germany; ${ }^{9}$ Universitätsklinik für Herz-und \\ Gefäßchirurgie, Bern, Switzerland; ${ }^{10}$ Universitätsklinikum, \\ Regensberg, Germany; ${ }^{11}$ Sana Herzchirurgie, Stuttgart, \\ Germany; ${ }^{12}$ UniversitätsKlinik Herzzentrum, Dresden, \\ Germany; ${ }^{13}$ Uniklinik Herzzentrum, Köln, Germany; ${ }^{14}$ Klinik für \\ Kardiochirurgie, Bad Neustadt, Germany; ${ }^{15}$ Policlinico S. Orsola \\ Malpighi, Bologna, Italy; ${ }^{16}$ Universitäts Medizin, Mainz, \\ Germany; ${ }^{17}$ Hospital Italiano, Buenos Aires, Argentina
}

Objective: The novel ACURATE TA ${ }^{\mathrm{TM}}$ Transapical Aortic Valve (Symetis, Switzerland) is composed of a porcine tissue valve sewn within a self-expanding nitinol stent covered by an anti-PV leak skirt designed for transapical implantation. Following its CEmark approval, a post-market multi-center registry collected safety and efficacy information from the first consecutive 250 treated patients with this new commercial device. Methods: The registry was conducted at 17 sites in Europe and South America to treat
250 high-risk elderly patients. This all-comers population presented pre-operatively with a mean aortic gradient of $43.3 \pm 17.4 \mathrm{~mm}$ $\mathrm{Hg}$, mean age of $80.9 \pm 6.3$ years, mean STS Score of $8.0 \pm 5.9 \%$ and mean Logistic EuroSCORE I of $22.3 \pm 12.7 \%$. The majority of patients (92.7\%) were in NYHA Class III/IV. All patients were treated at centers with a multi-disciplinary Heart Team. Results: The procedural success rate was $98.0 \%(n=245)$ with two valve-invalve procedures and three conversions to conventional surgery. The implantations were performed using small, medium and large bioprostheses covering an annular range of $21 \mathrm{~mm}$ to $27 \mathrm{~mm}$. The 30 days mortality and stroke rate is $6.8 \%$ and $2.8 \%$, respectively. Post-implant echocardiography revealed a mean gradient of $12.4 \pm 5.2 \mathrm{~mm} \mathrm{Hg}$ with a moderate paravalvular leak (Grade 2) in only $2.5 \%(n=6)$ of patients - all other patients demonstrated either none/trace or a $1+$ leak. A new permanent pacemaker was required in $10.0 \%(\mathrm{n}=25)$ of patients and $84.9 \%$ returning for follow-up at 30 days exhibited in NYHA Class I/II. Conclusions: The short-term registry results show a safety and efficacy profile comparable, if not improved in some aspects, to earlier approved TAVI valves. The device's ease-of-use is illustrated by the negligible learning curve as seen in the high procedure success rate. These real world data highlight excellent functional outcome with $97.5 \%$ of patients without relevant leak $(<2+)$ and a promising safety profile. The full 12 months results will be available early next year and will be presented at the Heart Valve Conference 2014.

\section{Impact of Aortic Valve Calcification Measured by Multidetector Computed Tomography on Survival in Patients with Aortic Stenosis: Results of an International Registry}

Marie-Annick Clavel ${ }^{1}$, Philippe Pibarot ${ }^{2}$, David Messika-Zeitoun ${ }^{3}$, Romain Capoulade ${ }^{2}$, Joseph Malouf', Phillip A. Araoz', Hector I. Michelena' ${ }^{1}$, Caroline Cueff ${ }^{3}$, Eric Larose ${ }^{2}$, Jordan D. Miller ${ }^{1}$, Alec Vahanian ${ }^{3}$, Maurice Enriquez-Sarano ${ }^{1}$

${ }^{1}$ Mayo Clinic, Rochester, MN, USA; ${ }^{2}$ Institut Universitaire de Cardiologie et de Pneumologie de Quebec, Quebec, QC, Canada; ${ }^{3} \mathrm{AP}-\mathrm{HP}$, Bichat Hospital, Paris, France

Objective: Aortic valve calcification (AVC) measures lesion severity of aortic stenosis (AS) and is useful for diagnostic purposes. Whether AVC predicts survival after diagnosis, independently of clinical and Doppler-echocardiographic AS characteristics, has not been studied. Our objective was to evaluate the impact of AVC on overall mortality under conservative treatment and without regards to treatment. Methods and Results: We enrolled in 3 academic centers 794 patients ( $73 \pm 12$ years, 274 women) diagnosed with AS by Doppler-echocardiography who underwent multi-detector-computed-tomography (MDCT) within the same episode of care. Absolute-AVC load and AVC density (ratio of absoluteAVC to left-ventricular-outflow-tract-area) were measured blinded to all other characteristics and severe AVC was separately defined for men and women. Indexed aortic-valve-area was $0.58 \pm 0.20$ $\mathrm{cm}^{2} / \mathrm{m}^{2}$, mean-gradient $35 \pm 19 \mathrm{~mm} \mathrm{Hg}$ and ejection fraction $60 \pm 12 \%$. During follow-up there were 440 Aortic-Valve-Replacements (AVR) and 194 deaths (115 under medical treatment). Uni- 
variable analysis showed strong association of absolute-AVC and AVC density to survival (both $\mathrm{p}<0.0001$ ) with spline-curve-analysis pattern of threshold and plateau of risk. Adjusting for age, gender, coronary-artery-disease, diabetes, symptoms, hemodynamic AS severity (indexed-aortic-valve-area and mean-gradient) and ejection fraction, severe absolute-AVC (adjusted-hazard-ratio: $1.75[1.04-2.92] ; \mathrm{p}=0.03$ ) or severe AVC density (adjustedhazard-ratio: 2.44 [1.37-4.37]; $\mathrm{p}=0.002$ ) independently predicted mortality under medical treatment with additive model predictive value (all $\mathrm{p} \leq 0.04$ ). Severe absolute-AVC (adjusted-hazard-ratio: 1.71[1.12-2.62]; $\mathrm{p}=0.01$ ) and severe AVC density (adjusted-hazard-ratio: 2.22 [1.40-3.52]; $\mathrm{p}=0.001$ ) also independently predicted overall mortality, after adjusting for time-dependent AVR. In patients with severe AVC, AVR was associated with marked mortality reduction (adjusted-hazard-ratio: 0.37[0.25-0.56]; $\mathrm{p}<0.0001$ ). Conclusions: This large multicenter outcome study of quantitative Doppler-echocardiographic and MDCT assessment of AS shows that measuring AVC load provides incremental survival prognostic value beyond clinical and Doppler-echocardiographic assessment. Severe AVC independently predicts excess mortality after AS diagnosis which is greatly alleviated by AVR. Thus, measurement of AVC by MDCT should be considered, not only for diagnostic but also for risk stratification purposes in patients AS.

\section{Importance of LV Outflow Tract Area Measured by Computed Tomography on Aortic Stenosis Survival}

Marie-Annick Clavel', Joseph Malouf', David Messika-Zeitoun ${ }^{2}$, Phillip A. Araoz ${ }^{1}$, Hector I. Michelena ${ }^{1}$, Maurice Enriquez-Sarano ${ }^{1}$

${ }^{1}$ Mayo Clinic, Rochester, MN, USA; ${ }^{2}$ AP-HP, Bichat Hospital, Paris, France

Objective: LV outflow tract (LVOT) is not circular and 2D echocardiography measurement may underestimate LVOT area and thus aortic valve area (AVA) calculation. However, this underestimation of AVA on survival after aortic stenosis (AS) diagnosis has not been addressed. The objective of this study was to assess the incremental value of AVA calculated with the use of LVOT area measured by Multi-Detector Computed Tomography (MDCT) over standard Doppler AVA calculation in determining survival under medical management. Methods: 269 AS patients underwent transthoracic Doppler-echocardiography and MDCT within an interval of 3 months. Aortic valve area (AVA) was calculated by Doppler (continuity equation) (AVAEcho) and by MDCT using direct measurement of LVOT area in the continuity equation (AVACT). Results: The patients were $76 \pm 11$ years old and $163(61 \%)$ were men. The mean AVAEcho was $0.94 \pm 0.32 \mathrm{~cm}^{2}$, AVACT $1.13 \pm 0.44 \mathrm{~cm}^{2}$, mean gradient $44 \pm 18 \mathrm{~mm} \mathrm{Hg}$ and LV ejection fraction $58 \pm 15 \%$. AVACT was larger than AVAEcho in 230 (86\%) patients with a mean difference of $0.19 \pm 0.20 \mathrm{~cm}^{2}$. During follow-up under medical management of $1.4 \pm 2.0$ years, there were 55 deaths. Two multivariate models were constructed, one with AVAEcho and one with AVACT and adjusted for age, gender, NYHA $\geq 3$, mean gradient and left ventricular ejection fraction. AVAEcho and AVACT were independent predictor of survival under medical treatment (HR: $1.18[1.05-1.34]$ per $0.10 \mathrm{~cm}^{2}$ de- crease; $\mathrm{p}=0.003$ and HR: $1.14[1.05-1.24]$ per $0.10 \mathrm{~cm}^{2}$ decrease; $\mathrm{p}=0.0008$, respectively) with similar accuracy of 2 models (all $\mathrm{p}=$ 0.11 by $\mathrm{C}$ statistics). However, the thresholds defining mortality were highly different: AVAEcho $\leq 1.00 \mathrm{~cm}^{2}$ (HR: 3.18[1.48-7.37]; $\mathrm{p}=0.002)$ and AVAC $\leq 1.20 \mathrm{~cm}^{2}$ (HR: 2.66[1.35-5.60]; $\left.\mathrm{p}=0.004\right)$ were independently predictive of mortality under medical treatment. Conclusions: AVA calculated using LVOT area measured by MDCT is larger than AVA by Doppler-echocardiography. However, the threshold predicting an excess mortality after AS diagnosis was also larger $\left(1.20 \mathrm{vs} .1 .00 \mathrm{~cm}^{2}\right)$ and the accuracy for risk stratification is equivalent.

\section{Long Term Outcome of Patients Undergoing Reintervention for Infective Endocarditis}

\section{Francesca Chiaramonti, Marco Solinas, Federica Marchi, Andrea Farneti, Sergio Berti, Enkel Kallushi, Tommaso Gasbarri, Mattia Glauber}

Heart Hospital G. Pasquinucci, Massa, Italy

Objective: Infective endocarditis (IE) are still a complication that affects the outcome of patients who undergo cardiac surgery. When IE occur is often required a reintervention. This study aimed to examine the outcome of patients undergoing reoperation for IE. Methods: From November 2001 to February 2013, 86 patients underwent reintervention for IE in our center. The first operation was an isolated mitral procedure (repair/replacement) in 13 patients, an isolated aortic procedure in 20 pts, an aortic replacement combined with a mitral procedure in 17 pts, a CABG combined with a valvular procedure in 16 patients and a complex operations in 20 pts. Thirty-two (37\%) patients was female. Mean age was $67.64 \pm 12.85$ and mean Logistic euroSCORE was $34.88 \pm 22.25$. The time between the first and second intervention was $149.95 \pm 65.33$ months. In 13 cases first operation was performed through a minimally invasive approach. Results: Overall in-hospital mortality was $19 \%$ (16). In two patients death was intraoperative. In $13(81 \%)$ cases death was due to low cardiac output related to sepsis; two (13\%) patients had a stroke, and one $(6 \%)$ patient had an abdominal hemorrhage. The mitral valve was involved to IE in 7 (44\%) patients who died in perioperative period. Mean cardiopulmonary bypass time and mean aortic cross clamp time was $205.92 \pm 86.17$ minutes and $136.06 \pm 71.83$ minutes. Mean Intensive Care Unit stay was $4.27 \pm 5.64$ days. Survival at 1 year, 5 -years and 10 -years were $63 \%, 55 \%$ and $48 \%$ respectively. Reoperation-free survival was $99 \%$; in one patients, ten years after first operation, was performed a Valve-in-Valve procedure with a Edwards Sapien Valve due to bioprosthesis degeneration. Follow up was completed of $85 \%$; median follow up time was 22 months (range 0-139 months). Conclusions: In our experience redo surgery for IE is more frequently in patients the previously had a combined procedure. The reoperations for IE are challenging for the surgeon and the in-hospital mortality rate is very high due to the underlying disease condition. Mitral valve IE is related to a higher risk of perioperative death. In survivors the freedom from IE recurrence is excellent. 


\section{Exercise-Induced Significant Increase in Mitral Pressure Gradient After Surgical Repair for Degenerative Mitral Regurgitation}

Kiyoshi Doi, Hitoshi Yaku, Kazunari Okawa, Sachiko Yamazaki, Tsunehisa Yamamoto, Suguru Ohira, Thetsuhiro Yamano

Kyoto Prefectural University of Medicine, Kyoto, Japan

Background: We occasionally encounter patients with considerably high mitral pressure gradient (PG) after mitral valve plasty (MVP) for degenerative mitral regurgitation (MR). The objective of this study was to assess these patients at rest and during exercise using echocardiography. Methods: Between April 2004 and March 2013, 247 consecutive patients underwent MVP for degenerative MR at our institution. From this cohort, we enrolled patients who met the following criteria: (1) those who underwent annuloplasty using a semi-rigid ring, (2) those with sinus rhythm at postoperative echocardiography and (3) those with a $<$ moderate residual MR. The final study group was composed of $67 \mathrm{pa}-$ tients. Of these patients, exercise stress echocardiography (ESE) was performed in 12 patients with good exercise tolerance. Mitral stenosis was defined as severe when the mean PG at rest and at peak exercise was $>10 \mathrm{~mm} \mathrm{Hg}$ and $>15 \mathrm{~mm} \mathrm{Hg}$, respectively. The indexed ring orifice area (IROA) was calculated by dividing the geometric orifice area of the used prosthetic ring by body surface area (BSA). Results: The ring size used was $30.7 \pm 3.3 \mathrm{~mm}(26-36$ $\mathrm{mm})$ and BSA was $1.67 \pm 0.2 \mathrm{~m}^{2}\left(1.3-2.1 \mathrm{~m}^{2}\right)$. IROA was $291.5 \pm 69.2$ $\mathrm{mm}^{2} / \mathrm{m}^{2}$. Resting mean mitral PG was $3.4 \pm 1.6 \mathrm{~mm} \mathrm{Hg}(1.0-8.9$ $\mathrm{mm} \mathrm{Hg}$ ). Peak exercise capacity according to ESE ranged from 75 to 150 Watts $(99.9 \pm 23.3$ Watts), which significantly increased mean mitral PG from $5.5 \pm 2.4 \mathrm{~mm} \mathrm{Hg}(2.1-9.5 \mathrm{~mm} \mathrm{Hg})$ at rest to $16.6 \pm 6.9 \mathrm{~mm} \mathrm{Hg}(7.8-29.9 \mathrm{~mm} \mathrm{Hg})$ at peak exercise. Although none of the 12 patients had severe mitral stenosis at rest, it became severe in 7 patients (mean mitral PG $>15 \mathrm{~mm} \mathrm{Hg}$ ) after exercise. Smaller IROA was well associated with higher mean mitral PG at peak exercise $\left(\mathrm{R}^{2}=-0.737 ; \mathrm{p}=0.006\right)$, and patients with higher mean mitral PG at peak exercise had lower exercise capacity $\left(\mathrm{R}^{2}=\right.$
$-0.620 ; \mathrm{p}=0.032)$. Conclusions: Even MVP using a true size ring may be associated with a high mitral PG when the ring size is relatively small for BSA, and this association becomes more prominent during exercise.

\section{Impact of Aortic Valve Replacement According to Echocardiographic Markers of Aortic Stenosis Severity}

Maxime Berthelot-Richer, Philippe Pibarot, Romain Capoulade, Jean G. Dumesnil, Abdelaziz Dahou, Florent Le Ven, Christophe Thebault, Marie-Annick Clavel

Quebec Heart and Lung Institute Research Center, Québec, QC, Canada

Background: Due to inherent discordance in the echocardiographic parameters cut-point used for severe aortic stenosis definition, discordant findings between aortic valve area $\left(\mathrm{AVA}<1.0 \mathrm{~cm}^{2}\right)$ and mean gradient ( $M G \leq 40 \mathrm{~mm} \mathrm{Hg}$ ) is a frequent finding. To reconciliate these parameters, some authors suggested that the AVA threshold for severe aortic stenosis definition should be lowered to $0.8 \mathrm{~cm}^{2}$. Our objective was to assess the survival benefit from aortic valve replacement (AVR) according to echocardiographic markers of AS severity: AVA, MG and peak aortic jet velocity $\left(\mathrm{V}_{\text {peak }}\right)$. Method: 837 patients with $\mathrm{AS}$ and normal flow (stroke volume indexed for body surface area $>35 \mathrm{~mL} / \mathrm{m}^{2}$ and left ventricular ejection fraction $\geq 50 \%$ ), who underwent a conservative treatment or an isolated AVR, were separated into 4 strata of AS severity based on AVA, MG or $\mathrm{V}_{\text {peak. }}$. We compared the effect of isolated AVR versus conservative therapy on survival in each strata of AS severity. A propensity score adjustment was used to eliminate covariate differences that may lead to biased estimates of treatment effect. Results: Mean AVA was $1.06 \pm 0.37 \mathrm{~cm}^{2}$, mean gradient $28.9 \pm 8.8 \mathrm{~mm} \mathrm{Hg}$ and mean $\mathrm{V}_{\text {peak }} 3.4 \pm 1.0 \mathrm{~m} / \mathrm{s} .286(34 \%)$ patients underwent an isolated AVR within 3 months following

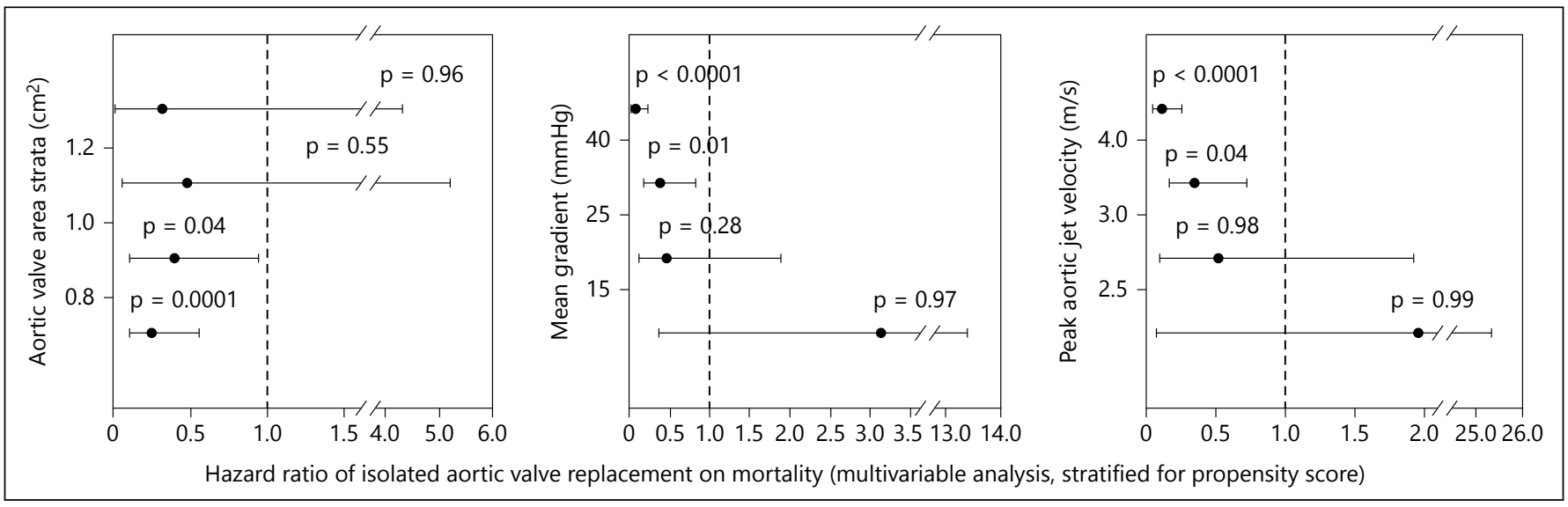

Fig. 1. Hazard Ratio for isolated Aortic Valve Replacement according to markers of Aortic Stenosis severity in Normal Ejection Fraction and Normal Flow AS patients (for Abstract of Maxime Berthelot-Richer). 
echocardiographic evaluation. During a mean follow-up of $4.4 \pm 3.0$ years there were 282 deaths. In multivariate analysis, patients with an AVA between 0.8 and $1 \mathrm{~cm}^{2}$ had a significant survival benefit from AVR (Hazard ratio of AVR $=0.40$ [0.10-0.94]; $\mathrm{p}=0.04$ ). Of note, a survival benefit from AVR was also observed in patients with MG between 25 to $40 \mathrm{~mm} \mathrm{Hg}$ or $\mathrm{V}_{\text {peak }}$ between 3 to $4 \mathrm{~m} / \mathrm{s}$ (figure 1). Conclusion: These results do not support the suggestion of decreasing AVA threshold value for severe AS to $0.8 \mathrm{~cm}^{2}$ and they confirm that AVR may be beneficial in a substantial number of patients with an AVA $<1 \mathrm{~cm}^{2}$ despite a $\mathrm{MG}<40 \mathrm{~mm} \mathrm{Hg}$ or a $\mathrm{V}_{\text {peak }}<4 \mathrm{~m} / \mathrm{s}$.

\section{Changes and Prognostic Significance of Stroke Volume After Transcatheter Aortic Valve Replacement}

\author{
Henrique B. Ribeiro, Florent Le Ven, Christophe Thébault, \\ Abdelaziz Dahou, Romain Capoulade, Haifa Mahjoub, \\ Marina Urena, Luis Nombela-Franco, Ricardo Allende, \\ Marie-Annick Clavel, Éric Dumont, Robert DeLarochellière, \\ Daniel Doyle, Josep Rodés-Cabau, Philippe Pibarot
}

Quebec Heart \& Lung Institute, Quebec, QC, Canada

Background: The presence of low LV outflow, i.e. reduced stroke volume index $(\mathrm{SVi})$, prior to the procedure has been recently shown to be a powerful independent predictor of early and late mortality in high-risk patients with severe aortic stenosis (AS) undergoing transcatheter aortic valve replacement (TAVR). The objectives of this study were to examine changes in flow (i.e. SVi) occurring after TAVR, their determinants and the impact of postprocedural SVi (PP-SVi) on mortality. Methods: We retrospectively analyzed the clinical, Doppler-echocardiographic, and outcome data prospectively collected in 255 patients who underwent TAVR for symptomatic severe AS. Echocardiograms were performed before (baseline), within 5 days after procedure (post-procedural) and 6 months to 1 year following TAVR (late-FU). Results: Post-TAVR echocardiographic data were available in 255 (89\%) patients. Mean follow-up was $18 \pm 15$ months. Patients with early post-procedural SVi (PP-SVi) $<35 \mathrm{ml} / \mathrm{m}^{2}(\mathrm{n}=138 ; 54 \%)$ had increased mortality (HR: 1.97, $\mathrm{p}=0.003$ ) compared to those with PP-SVi $>35 \mathrm{ml} / \mathrm{m}^{2}$ ( $\left.\mathrm{n}=117 ; 46 \%\right)$. Furthermore, patients with baseline (pre-procedural) SVi (B-SVi) $<35 \mathrm{ml} / \mathrm{m}^{2}$ and PP-SVi $>35 \mathrm{ml} / \mathrm{m}^{2}$, i.e. with normalized flow, had better survival (HR: 0.46 , $\mathrm{p}=0.03)$ than those with both B-SVi and PP-SVi $<35 \mathrm{ml} / \mathrm{m}^{2}$, i.e persistent low-flow, and similar survival compared to those with both B-SVi and PP-SVi $>35 \mathrm{ml} / \mathrm{m}^{2}$, i.e. maintained normal flow. In a multivariable model adjusted for gender, atrial fibrillation, trans-apical/transfemoral approach and post-procedural echo parameters (LVEF, mean gradient, pulmonary hypertension, moderate or severe aortic and mitral regurgitations), PP-SVi was independently associated with increased risk of mortality (HR 1.41 per $10 \mathrm{ml} / \mathrm{m}^{2}$ decrease, $\mathrm{p}=0.03$ ). The pre-procedural factors associated with PP-SVi $<35 \mathrm{ml} / \mathrm{m}^{2}$ were: atrial fibrillation (OR: $2.55, \mathrm{p}=$ 0.01 ), trans-apical approach (OR: $2.46, \mathrm{p}=0.002)$, mean transvalvular gradient (OR: 1.22 per $10 \mathrm{~mm} \mathrm{Hg}$ decrease, $\mathrm{p}=0.05$ ), and indexed aortic valve area (OR: 1.67 per $0.1 \mathrm{~cm}^{2} / \mathrm{m}^{2}$ decrease, $\mathrm{p}=$
0.02). Conclusion: The persistence of low-flow early after the procedure is an independent predictor of late mortality following TAVR.

\section{Mid-Term Outcomes of Suture Annuloplasty Versus Ring Annuloplasty for Functional Tricuspid Regurgitation}

\author{
Hiroki Hata, Tomoyuki Fujita, Yusuke Shimahara, Shunsuke Sato, \\ Junjiro Kobayashi
}

National Cerebral and Cardiovascular Center, Suita, Japan

Objective: There are some good evidences supporting ring annuloplasty over suture annuloplasty for patients with functional tricuspid regurgitation (TR). However, it is still controversial whether there is a significant difference in surgical outcome between the two techniques. The purpose of this study was to compare the mid-term results of tricuspid annuloplasty (TAP) with or without an annuloplasty ring. Methods: From January 1996 to May 2013, 558 patients (mean age, $64.7 \pm 9.1$ years, $59.6 \%$ women) underwent TAP for functional TR at our institution, of whom 324 underwent conventional suture annuloplasty with De Vega or Kay technique and the other 234 underwent ring annuloplasty (flexible ring, 59; rigid ring, 175). Transthoracic echocardiography was performed before and 1 week after operation and in the follow-up period. The severity of TR was graded from 0 to 4 . The clinical data were retrospectively collected and statistically analyzed. Results: The mean follow-up period was 72.4 (1-208) months. Suture annuloplasty patients were younger (62.9 versus 67.3). Other preoperative characteristics and operative parameters were similar between the two groups. There was no significant difference in the overall survival (suture $96.7 \%$ versus ring $98.2 \%$ at 1 year, $89.7 \%$ vs. $91.5 \%$ at 5 years, $83.4 \%$ vs. $87.6 \%$ at 10 years, respectively) and freedom from recurrent moderate to severe TR (99.6\% vs. $99.1 \%$ at 1 year, $96.6 \%$ vs. $86.6 \%$ at 5 years, $84.9 \%$ vs. $81.1 \%$ at 10 years, respectively) between the groups; however TR grade was significantly better in the ring group at discharge $(1.2 \pm 0.9$ vs. $0.9 \pm 0.8)$ and follow-up ( $1.6 \pm 0.9$ vs. $1.1 \pm 0.8)$. Multivariate analysis revealed that suture annuloplasty (odds ratio 3.45 ), prior cardiac surgery (odds ratio 2.60) and preoperative severe TR (odds ratio 3.51) were the predictors of recurrent TR. There was no significant difference in mid-term outcomes between the patients with a flexible ring and a rigid ring. Conclusions: In conclusion, both suture and ring annuloplasty provide acceptable mid-term survival and stable postoperative regurgitation grade. Suture annuloplasty, prior cardiac surgery and preoperative severe TR were the risk factors of recurrent TR. 


\section{In Vitro Modulation of Human Immune Responses of Cryopreserved Aorta and Heart Valves by Vitrification Treatment}

\author{
Benjamin A. Hoegerle ${ }^{1}$, Martina Seifert ${ }^{2}$, Karin Sudrow ${ }^{2}$, \\ Isabella Werner ${ }^{3}$, Nadija Souidi ${ }^{2}$, Meagan Stolk ${ }^{2}$, \\ Kelvin G.M. Brockbank ${ }^{4}$, Andres Beiras ${ }^{1}$, Anton Moritz ${ }^{1}$, \\ Ulrich A. Stock ${ }^{1}$ \\ ${ }^{1}$ University Hospital Frankfurt, Frankfurt am Main, Germany; \\ ${ }^{2}$ Charité Universitätsmedizin Berlin, Berlin, Germany; \\ ${ }^{3}$ University Hospital Frankfurt, Frankfurt, Germany; \\ ${ }^{4}$ Department of Regenerative Medicine and Cell Biology, \\ Medical University of South Carolina, Charleston, SC, USA
}

Introduction: Current challenge of allogenic heart valves is minimizing inflammation and rejection. The present in vitro study analyzed whether treatment of cryopreserved human aortic valves by vitrification solution modulates immune responses. Methods: 5 human aortic valves were frozen and thawed according to protocols of conventional cryopreservation (CFC). Additionally tissue was thawed and treated by vitrification solution $(\mathrm{CFC}+\mathrm{V})$. Tissue punches $(n=6)$ were co-cultured for 7 days with human peripheral blood mononuclear cells (PBMC) to quantify induced proliferation by flow cytometry. Co-cultures with sorted CD14+ monocytes were analyzed for activation, differentiation and polarization into macrophages. Release of cytokines by cytometric bead array was determined. Results: In all matrix/PBMC settings we found neither induction of immune cell proliferation nor release of all tested pro-inflammatory cytokines compared to controls with mitogen-activate cells. Co-cultures with CD14+ monocytes showed marginal increase in proportion of CD206+ cells for both groups, however no increase regarding fraction of CCR7 expressing cells. Percentage was higher only in co-cultures with $\mathrm{CFC}+\mathrm{V}$ aorta but not in valves. In all co-cultures IL-6 was exclusively detected, showing reduced levels only in aortic co-cultures of $\mathrm{CFC}+\mathrm{V}$ aortic tissue compared to CFC. Conclusion: Our data emphasize potential of vitrification to modify immunogenic properties of allogenic aortic and heart valve tissue. Increase of proportion of macrophages with a more anti-inflammatory phenotype and decrease of IL-6 levels for aortic tissue by vitrification treatment might offer modulation of immune responses in general. Vitrification is easy, fast, and safe preserving biological tissues and promoting better clinical graft performance.

\section{Novel Tissue Technology Platform Improves Durability and Valve Function Over Current Standard Bovine Pericardial Valves}

\section{Bart Meuris, Erik Verbeken, Willem Flameng}

KU Leuven, Leuven, Belgium

Objective: Use of bioprosthetic heart valves is limited in younger patients because of early progressive structural valve deterioration. To address this problem, a novel tissue technology platform was developed using stable functional group capping and preservation by glycerolization. The aim of this study was to evaluate the hemodynamic performance and durability of pericardial valves using this novel platform in a chronic ovine model. Methods: Forty-five juvenile sheep were prospectively randomized to receive a bovine pericardial mitral valve replacement (CarpentierEdwards PERIMOUNT, model 6900P; Edwards Lifesciences, Irvine, CA) with (TEST) or without (CONTROL) the novel tissue technology platform. All valves were size $25 \mathrm{~mm}$. Transthoracic echocardiography was performed at 1 week and 8 months postoperatively. All animals were sacrificed at 8 months. Perimount valves were analyzed radiographically, histologically, and chemically for calcium content. Animals that died during the operation, or that developed serious infections leading to early death, were excluded from analysis. Results: Thirty-one animals (TEST, $\mathrm{n}=$ 17; and, CONTROL, $\mathrm{n}=14$ ) survived to 8 months. At 1 week, transthoracic echocardiography demonstrated normal valve function in both groups. At 8 months, cardiac output was significantly increased to the same extent in both groups. The mean pressure gradient was significantly lower in the TEST compared to the CONTROL, 4.0 vs. $5.6 \mathrm{~mm} \mathrm{Hg}(\mathrm{p}=0.04)$. Leaflet mobility was greater in the TEST compared to the CONTROL, with greater turbulence observed in the CONTROL. Compared to the CONTROL, calcium content measured was significantly reduced in the TEST $(2.0 \pm 0.3$ vs. $6.8 \pm 1.6 \mu \mathrm{gm} / \mathrm{mg}, \mathrm{p}=0.002)$, supported by Faxitron analysis and histology. Histology also demonstrated less leaflet pannus formation in the TEST group. Conclusions: The results from this prospective, randomized chronic ovine study demonstrated significantly improved hemodynamic performance, leaflet mobility, and reduced calcium content with a trend toward reduced pannus formation, after mitral valve replacement with bovine pericardial valves using a novel tissue technology platform.

\section{Aortic Valve Replacement in Patients 80 Years Old and Younger. Comparison of Mid-Term Outcome Between Surgical Implantation with Stentless Bio-Prosthesis and Trans-Cutaneous Implantation-(TAVI)}

Rephael Mohr, Ariel Finkelstein, Yanai Ben-Gal, Amir Kramer, Arie Steinvil, Dmitri Pevni

Sourasky medical center, Tel Aviv, Israel

Background: Most trans-cutaneous aortic valve implantation (TAVI) procedures performed today are done in patients older than 80 . The purpose of these report is to compare early outcome and midterm survival of TAVI, to that of surgically implanted stentless aortic valve replacement (AVR) for patients 80 years old and younger. Methods: One hundred and four consecutive TAVI patients 80 years old and younger were implanted from January 2009 to May 2013. They were compared to 201 AVR patients 80 years old and younger, operated on between 1997-2013. Inclusion criteria was severe aortic stenosis ( $\min \mathrm{EOA}=\mathrm{BSAx} 0.85)$. Patients with bacterial endocarditis and patients with aortic root enlargement were excluded from the AVR group. Results: AVR patients were younger $(69+8$ vs. $76+14, p<0.001)$. However occurrence of female gender, peripheral vascular disease and congestive heart 
failure were similar. More patients in the TAVI group had chronic renal failure (CRF) $(27.9 \%$ vs. $9.5 \%$, p < 0.001), more had chronic obstructive lung disease (COPD) $(28.8 \%$ vs. $5.5 \%, \mathrm{p}<0.001)$ and more had prior coronary artery bypass surgery (CABG) $(27 \%$ vs. $6 \%, \mathrm{p}<0.001)$. Postoperatively, more patients in the TAVI group suffered stroke $(12.5 \%$ vs. $3.5 \%, \mathrm{p}=0.003)$. Three years survival (Kaplan-Meier) of the AVR patients was better (95.2\% vs. $83.7 \%$, $\mathrm{p}=0.016$ log-rank test). Ejection fraction $\leq 30 \%$ and diabetes melitus were the only independent predictors of decreased survival. Assignment to TAVI or AVR was not found to be a significant risk factor for decreased survival (Cox model). Conclusions: Midterm outcome of AVR patients 80 years old or younger is better. However, TAVI may be considered in these patients with aortic stenosis, especially in patients after prior CABG or patients with comorbidities such as COPD and CRF, despite increased risk of stroke.

\section{Psycho-Emotional Status and Health-Related Quality of Life in Patients Following Cardiac Valve Surgery}

\author{
Tali Bayer-Topilsky' ${ }^{1}$ Maurice Enriquez-Sarano ${ }^{2}$ \\ ${ }^{1}$ Myers-JDC-Brookdale, Jerusalem, Israel; ${ }^{2}$ Mayo Clinic, \\ Rochester, MN, USA
}

Objective: Severe MR is associated with low psycho-emotional status (PES). Yet, it is unknown whether this association is causal and whether surgical correction affects PES. The current study assessed whether a causal association exists between severe mitral regurgitation (MR) and PES and whether suppression of MR by surgery improves PES and Health-Related Quality of Life (HR-QoL). Methods: 131 patients (mean age, 60, male, 77\%) undergoing MR surgery, 62 with MR treated conservatively and 35 with normal echocardiogram, were prospectively assessed with comprehensive measures of MR, PES (anxiety, posttraumatic symptoms (PTS) and depression) and HR-QoL at baseline and at post-operative 6 months follow-up. Results: Baseline PES scores were poorer in surgical group compared to normal controls (anxiety, $\mathrm{p}=0.01$; PTS, $\mathrm{p}=$ $0.02)$. Improvement was noted only in the mitral-operated group (all $\mathrm{p} \leq 0.02$ ), and the follow-up assessment showed similar scores among all groups (all $\mathrm{p} \geq 0.4$ ). Improvement with operation was independent of heart failure symptoms at baseline and was different between groups adjusted for symptoms (all $\mathrm{p}<0.02$ adjusted). Baseline HR-QoL of patients in the surgical group were also poorer compared to other groups (LASA, all $\mathrm{p}<0.005 ;$ SF-12 Physical, all $\mathrm{p}<0.001$ ). These measures improved significantly after surgery (all $\mathrm{p} \leq 0.0002)$ and resembled those of the other groups at follow up (all $\mathrm{p}>0.06$ ). Improvement in PES and HR-QoL were not related to the surgical approach (robotic vs. standard, all $p \geq 0.2$ ). Conclusions: Association between severe MR and poor PES is causal. Mitral valve surgery normalizes PES and HR-QoL, and the improvement is an added benefit of MR correction.

\section{Alterations in Substrate Stiffness and Composition Effect the Progression of Calcific Aortic Valve Disease}

\author{
Lauren Baugh ${ }^{1}$, Irene Georgakoudi ${ }^{2}$, Phillip Hinds ${ }^{3}$ \\ Gordon Higgins ${ }^{4}$, Lauren Black ${ }^{2}$
}

${ }^{1}$ Tufts University, Medford, MA, USA; ${ }^{2}$ Department of Biomedical Engineering, Tufts University, Medford, MA, USA; ${ }^{3}$ Molecular Oncology Research Institute, Tufts Medical Center, Boston, MA, USA; ${ }^{4}$ Molecular Cardiology research institute Center forTtranslational Genomics, Tufts Medical Center, Boston, MA, USA

Objective: To test an in vitro model of calcific aortic valve nodule growth that modulates stiffness and substrate composition in polyacrylamide (PAAM) gels to understand progression of calcif-

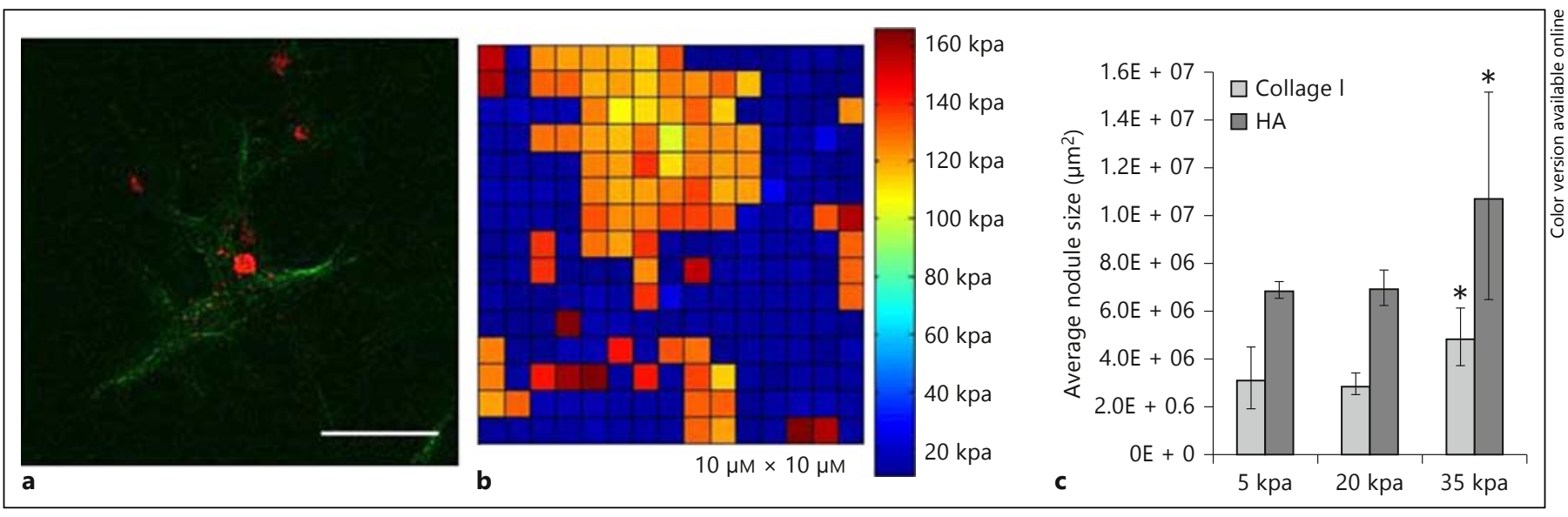

Fig. 1. a) Non-linear confocal microscopy image of calcium nodule formation by VICs on a PAAM gel. Green represents the SHG signal generated by collagen fibers while red represents the TPEF from the calcium nodules. b) AFM force volume plot exhibiting the change in matrix stiffness with the formation of a calcium nodule in murine valve leaflet. c) Average size of nodules grown on PAAM gels with $\mathrm{HA}$ and collagen binding sites (for Abstract of Lauren Baugh). 
ic aortic valve disease (CAVD). Methods: Rat aortic valves were isolated and used in an explant culture to obtain valve interstitial cells (VICs). The isolated VICs were used to seed PAAM gels created at three levels of stiffness: $5 \mathrm{kPa}, 20 \mathrm{kPa}$, and $35 \mathrm{kPa}$. These stiffness values were chosen to model normal valve stiffness $(5 \mathrm{kPa})$, a valve with extensive CAVD $(35 \mathrm{kPa})$, and a transitional valve stiffness $(20 \mathrm{kPa})$. We also incorporated either collagen I or hyaluronic acid (HA) as binding sites to distinguish compositional effects that occur with remodeling. To induce calcification, transforming growth factor $-\beta 1$ (TFG- $\beta 1$ ) was added to the culture medium. Additional murine leaflets were isolated and placed in culture media with TFG- $\beta 1$. Results: Our model system accurately recapitulates the development of calcified nodules in both human and murine CAVD as demonstrated through two-photon excited fluorescence (TPEF) spectral data. As demonstrated in figure 1, nodule formation and collagen I reorganization were measured using TPEF and the second harmonic generation (SHG) signal from the collagen fibers, respectively (figure 1a); in figure $1 \mathrm{~b}$, elastic modulus was measured in the PAAM gels and the isolated murine leaflets using atomic force microscopy (AFM); and nodule growth was analyzed using the calcium fluorescent indicator fluo-4. Stiffness had a significant $(\mathrm{p}<0.05)$ impact on nodule size (figure 1c). Additionally, the development of nodules exacerbated nodule growth by further increasing matrix stiffness and altering the matrix structure indicated through disorganized collagen fibers demonstrated with SHG. Conclusions: VIC activation to myofibroblasts after TFG- $\beta 1$ stimulation was enhanced with increasing substrate stiffness and alterations in substrate composition. Our model allows for identification of calcification in real time and provides a visual representation of changes in the surrounding substrate in response to nodule formation.

\section{Surgery for Acute Decompensated Mitral Valve Disease: Single Centre Experience of 292 Patients}

\author{
Sergey A. Leontyev, Piroze Davierwala, Martin Schneefogt, \\ Farhad Bakhitiary, David Holzhey, Michael A. Borger, \\ Martin Misfeld, Friedrich W. Mohr
}

Heart Center, University of Leipzig, Leipzig, Germany

Objective: Urgent and emergent mitral valve (MV) surgery performed for acute decompensated MV disease is usually associated with an increased risk. We analyzed our early and late results, and determined the independent predictors of early and late mortality. Methods: From 04/95 until 06/10 292 patients underwent MV surgery for acute decompensated MV disease, which was defined as patients presenting with dyspnea or tachycardia with pulmonary congestion or edema, and/or cardiogenic shock. Patient age was $64 \pm 11$ years; $47.6 \%$ were female. Twenty percent of patients were in cardiogenic shock, $27 \%$ required inotropic support and $12 \%$ were intubated at the time of surgery. Acute endocarditis was the primary indication for surgery in $61 \%$ of patients. Mean follow-up was $6.1 \pm 0.4$ years (range $0-14$ years) for all patients. Results: Overall 30-day mortality was 20.2\%. NYHA class IV (OR 2.1, $\mathrm{p}=0.02,95 \%$ CI 1.1-4.2) and cardiogenic shock (OR 5.1, $\mathrm{p}<0.01$, $95 \%$ CI 2.0-12.5) were identified as independent predictors of 30- day mortality. Cardiogenic shock (OR 2.9, p $<0.01,95 \%$ CI 1.65.1 ), preoperative inotropic support (OR 1.9, p $<0.01,95 \%$ CI $1.1-$ 3.3 ), preoperative dialysis (OR $2.5, \mathrm{p}=0.02,95 \%$ CI 1.1-1.4), diabetes mellitus (OR 1.7, $\mathrm{p}=0.04,95 \% \mathrm{CI} 1.02-2.9)$ and preoperative NYHA class IV (OR 1.2, p $=0.01,95 \%$ CI 1.04-1.3) were independent predictors of long-term mortality. Mean survival at 1,5 and 10 years was $61 \pm 3 \%, 52 \pm 3 \%$ and $28 \pm 5 \%$, respectively. Conclusions: Surgery for decompensated MV disease is associated with high early and long-term mortality, especially in patients with cardiogenic shock and advanced congestive heart failure. Efforts should be made to refer patients for MV surgery before the onset of advanced symptoms or congestive heart failure.

\section{How Can Complex Statistical Models Help Cardiologists with VHD Patient Prognostication? The Example of Allograft Valve Function to Predict Patient Outcome}

\section{Eleni-Rosalina Andrinopoulou, Dimitris Rizopoulos, Emmanuel Lesaffre, Johanna Takkenberg}

Erasmus Medical Center, Rotterdam, The Netherlands

Background: In the prediction of prognosis for new patients suffering from severe heart valve disease, cardiologists consider patient characteristics, echocardiographic measurements (e.g. aortic gradient AG and aortic regurgitation AR), to estimate survival and freedom from reoperation. Intuitively, cardiologists adjust their prognosis over time, with the change in clinical status of the patient at each clinic visit. This study aims to illustrate dynamic predictions using all available information. Methods: In our study, 270 patients after allograft aortic valve replacement were followed for 20 years. To analyze patient characteristics, longitudinal echocardiographic and survival data and to use this information to derive survival and freedom from reoperation predictions, we used the joint modeling framework. This approach correlates the AG and AR progression with survival and freedom from reoperation in different ways, e.g.: 1) AG and AR values at specific time points, 2) AG and AR values and their directions at specific time points and 3) the whole $A G$ and $A R$ evolutions up to specific time points. Furthermore, different patient characteristics may play an important role. Thus, we derive predictions for a new patient assuming different models. Finally, since selecting a single model ignores model uncertainty we also obtain weighted predictions. Results: Figure 1, which presents the predictions using different models for a future patient, shows a variation of results we obtained. From the combined predictions we observed that different models are appropriate for different patients but also for different time points within the same patients. Specifically for the future patient, we observe a contribution of Model 4 and Model 6 in the predictions. Conclusions: Every patient is unique and assuming same prediction models for all patients may not be appropriate. The proposed approach provides the cardiologist a useful tool to assess the impact of AG and AR on patient prognosis. Although the concept needs to be investigated further, it could eventually serve as an early warning system, allowing the necessary time for the cardiologist to plan an intervention. 


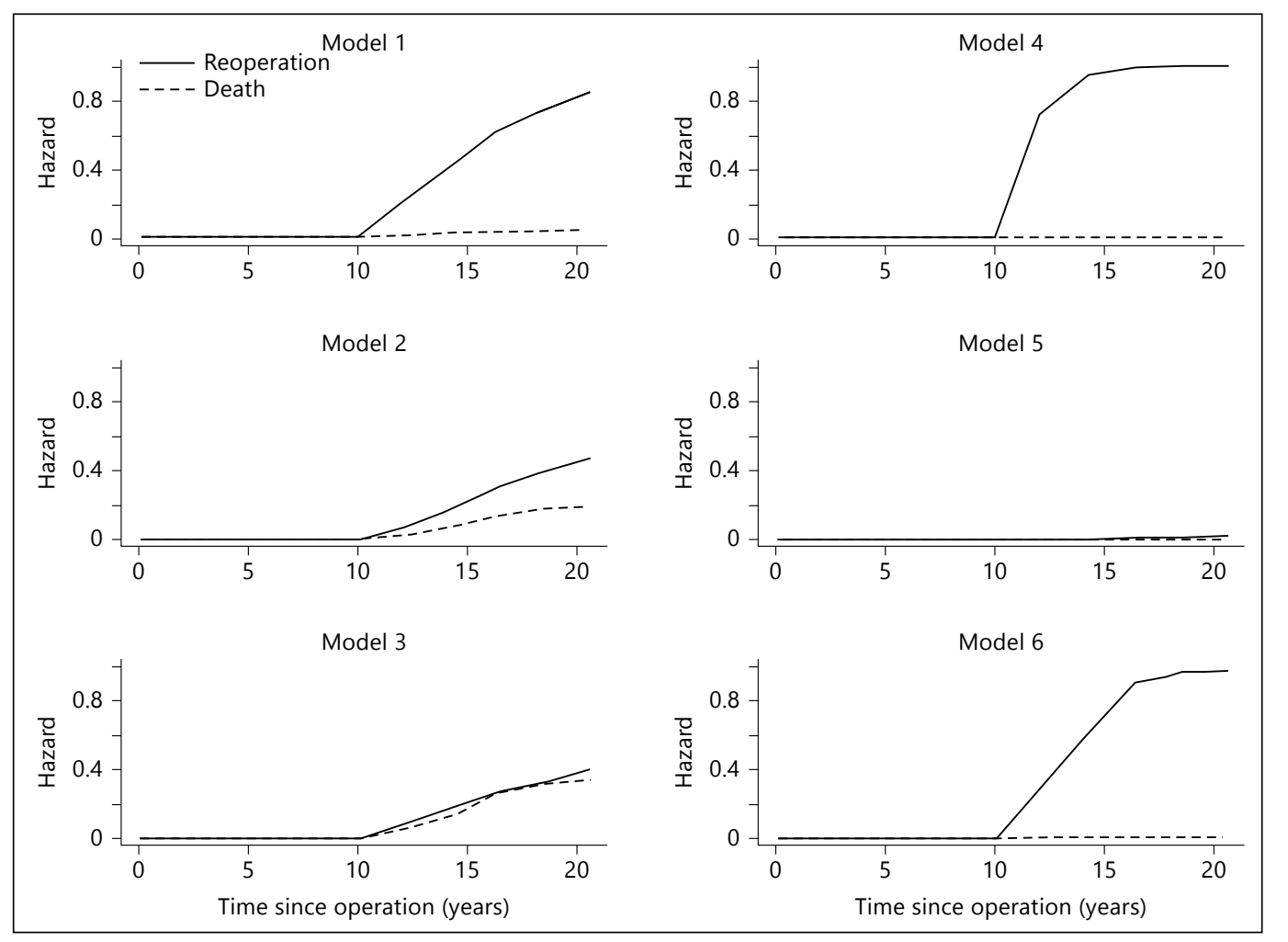

Fig. 1. Prediction plots for a future patient after his last follow-up visit at time $=10$ assuming six different models. Each row represents a different association structure for the longitudinal and each column assumes different patient characteristics. Model 1 and Model 4 assume the first way of linking longitudinal and survival data 1) mentioned in the Methods. Model 2 and Model 5 the 2) and Model 3 and Model 6 the 3). Moreover, Model 1, Model 2 and Model 3 include the baseline covariates: age and gender, while Model 4, Model 5 and Model 6 the baseline covariates: age, gender, LV fraction, donor age and diameter (for Abstract of Eleni-Rosalina Andrinopoulou).

\section{Osteopontin - CD44v6 Interaction Mediates Calcium Deposition via Phospho-Akt in Valve Interstitial Cells from Patients with Non-Calcified Aortic Valve Sclerosis}

Paolo Poggio ${ }^{1}$, Emanuela Branchetti ${ }^{1}$, Juan B. Grau' ${ }^{1}$, Robert C. Gorman ${ }^{1}$, Joseph H. Gorman III', Micheal S. Sacks², Joseph E. Bavaria ${ }^{1}$, Giovanni Ferrari ${ }^{1}$

${ }^{1}$ University of Pennsylvania, Philadelphia, PA, USA;

${ }^{2}$ University of Texas at Austin, Austin, TX, USA

Objective: Calcific Aortic Valve Disease (CAVD) is the most frequent valve disorder associated with surgical aortic valve replacement (AVR) in the US. With shifting demographics from an aging population, AVR is projected to reach more than 850,000 cases worldwide by 2050, from today's 300,000 . While the final stages of the disease (Aortic Valve Stenosis) are well described, the cellular mechanisms responsible for the initial phases (Aortic Valve Sclerosis) remain largely unknown due to its subclinical presentation. In addition, sclerotic tissues are generally not available to investigators since these valves are not replaced until severe stenosis occurs. Osteopontin (OPN) is a phosphorylated-acidic-gly- coprotein that accumulates within the aortic leaflets and labels valve interstitial cells (VICs) activation even in non-calcified asymptomatic patients. In recent works, we - and others - have demonstrated the capacity OPN to protect VICs against ectopic and dystrophic calcification. Here we unveil the specific interaction of OPN with CD44v6 and the related intracellular signaling pathway controlling calcium deposition in human VICs. Methods: Upon informed consent 23 patients and 4 controls were enrolled through the cardiac surgery and heart transplant programs. Human aortic valves and VICs were obtained and tested, for osteogenic transdifferentiation, ex vivo and in vitro, respectively. A uniaxial tensile bioreactor was used to test the effects of biomechanical stimulation of surgically resected tissues. OPN-CD44 functional interaction was analyzed using Proximity Ligation Assay and the intracellular signaling pathways were investigated. Results: First we demonstrated a specific OPN-CD44 functional interaction in early asymptomatic stage of CAVD. We then show that preventing OPNCD44 interaction result in calcium accumulation of VIC obtained from non-calcified AVSc patients after treatments with BMP4. Finally, using neutralizing antibodies and general inhibitors of intracellular signaling pathways we unveil that Akt phosphorylation induced by OPN-CD44 is required to protect human sclerotic VICs from calcium deposition. Conclusions: Here we unveil a spe- 
cific protein-protein association and intracellular signaling mechanisms of OPN, one of the early markers of CAVD. Understanding the molecular mechanisms of early VIC activation and calcium deposition in asymptomatic stage of CAVD could open new prospective for diagnosis and therapeutic intervention.

\section{Predictions of Wall-Shear Stress on the Aortic Valve Leaflets Using a Three-Dimensional Fluid-Structure Interaction Model}

\section{Kai Cao Philippe Sucosky}

University of Notre Dame, Notre Dame, IN, USA

Background: While hemodynamic stresses have been shown to modulate valvular biology, the native valvular fluid shear stress environment remains largely unknown. The objective of the pres- ent study was to characterize the regional wall shear stress (WSS) on aortic valve leaflets using three-dimensional (3D) fluid-structure interaction (FSI) modeling. Methods: The idealized 3D model consisted of the aortic root, the aortic sinus and three identical compliant leaflets with physiologic dimensions and non-uniform thickness. Only $1 / 6$ of the structure was considered due to the valve trileaflet symmetry. The fully coupled FSI simulations were run in ANSYS by imposing a physiologic transvalvular pressure at the inlet of the fluid domain. Local leaflet WSS was characterized in terms of temporal shear magnitude (TSM) and oscillatory shear index (OSI). Results: The model successfully predicted leaflet and blood flow dynamics at a mean physiologic cardiac output of 4.2 $\mathrm{L} / \mathrm{min}$. During systole, the positive transvalvular pressure gradient generates a central forward flow contributing to leaflet opening (fig. $1 \mathrm{a}-\mathrm{c})$. During diastole, inversion of the pressure gradient produces strong flow recirculation and vortical structures in the aortic sinus cavity, which promotes leaflet coaptation and valve closure (fig. 1d-e). Regardless of the leaflet region (base, belly, tip), the leaflet WSS was shown to be dominated by the radial component (fig. 1f). The WSS magnitude on the ventricularis (TSM: 10.30
Fig. 1. Snapshots of flow velocity field computed at different phases (a-e). WSS variations in the leaflet base, belly and tip (f) (for Abstract of $\underline{\mathrm{Kai} \mathrm{CaO}}$ ).

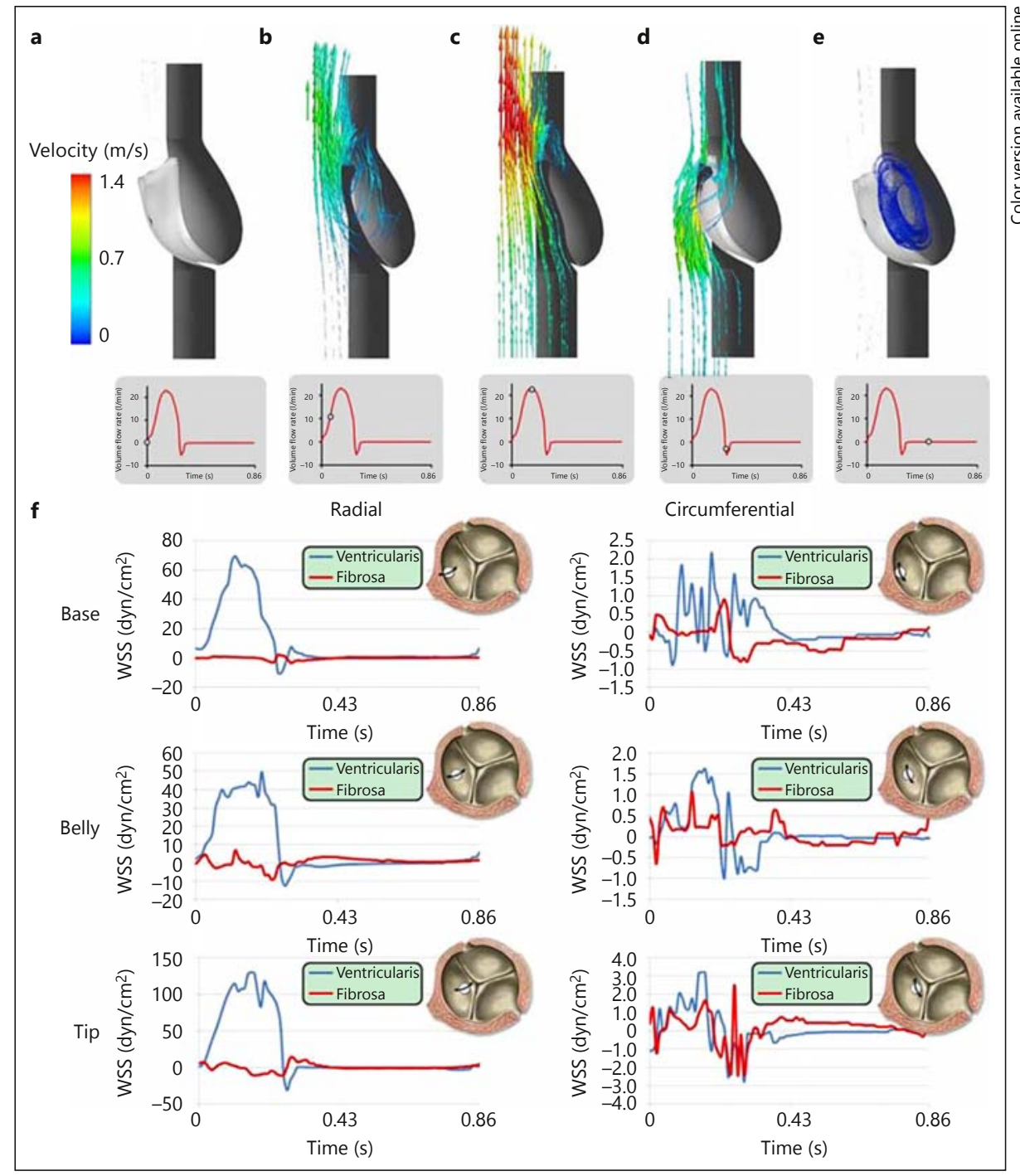


$\mathrm{dyn} / \mathrm{cm}^{2}$ ) was found to be significantly higher than on the fibrosa (TSM: $1.83 \mathrm{dyn} / \mathrm{cm}^{2}$ ). The radial WSS variations predicted on both leaflet surfaces indicated the existence of a unidirectional WSS on the ventricularis $(\mathrm{OSI}=0.04)$ and a bidirectional WSS on the fibrosa (OSI $=0.49$ ). Conclusion: This study is the first to implement a commercial FSI software to predict the regional WSS on aortic valve leaflets. The results provide new insights into the macro-scale valvular hemodynamics and are critical to the assessment of the hemodynamic theory of calcific aortic valve disease.

\section{Role of Pathologic Fluid Shear Stress in Early Development of Calcific Aortic Valve Disease}

\author{
Ling Sun Philippe Sucosky \\ University of Notre Dame, Notre Dame, IN, USA
}

Background: Calcific aortic valve disease (CAVD) is a disorder presumably triggered by interplay between cardiovascular risk factors and hemodynamic cues. While studies demonstrated that progressive alterations in fluid shear stress (FSS) on the fibrosa could trigger leaflet inflammation, the mechanisms of CAVD pathogenesis secondary to side-specific FSS abnormalities are poorly understood. The objective of this study was to characterize ex vivo the contribution of isolated and combined abnormalities in FSS magnitude and frequency to early valvular pathogenesis, and to investigating the respective role played by BMP- 4 and TGF- $\beta 1$ in valvular pathogenesis in response to pathologic shear stress levels. Methods: Porcine leaflets were subjected to sub-physiologic/physiologic/supra-physiologic FSS magnitude and frequency for 24, 48 and 72 hours in a double cone-and-plate device. Endothelial activation and paracrine signaling were investigated in terms of cell-adhesion molecules (ICAM-1, VCAM-1) and cytokines (BMP-4 and TGF- $\beta 1$ ), respectively. Extracellular matrix (ECM) degradation was characterized by measuring the expression and activity of MMPs and cathepsins. Pro- and anti-osteogenic media were used to characterize the role of cytokines (BMP-4 and TGF- $\beta 1$ ) in the FSS-induced pathological response. Results: The effect of the FSS treatment yielding the most significant pathological response was examined over a 72hour period to characterize the time-dependence of FSS mechanotransduction. While cytokine expression was stimulated under elevated FSS magnitude at normal frequency, ECM degradation was stimulated under both elevated FSS magnitude at normal frequency and physiologic FSS magnitude at abnormal frequency. In contrast, combined FSS magnitude and frequency abnormalities maintained valvular homeostasis. The pathological response under supra-physiologic FSS magnitude peaked at 48 hours but was then maintained until the 72-hour time point. Under non-physiologic FSS magnitude, TGF- $\beta 1$ inhibition suppressed FSS-induced ECM degradation, while BMP-4 inhibition and supplementation had limited effects on the FSS-induced biological response. Conclusions: This study confirms the sensitivity of valve leaflets to FSS magnitude and frequency and suggests the ability of supra-physiologic FSS levels or abnormal FSS frequencies to initiate CAVD pathogenesis. The results related to TGF- $\beta 1$ inhibitor in response of pathological FSS provide new evidence about the transduction of valvular hemodynamic alterations into a pathological response.

\section{Tissue Engineered Atrioventricular Valve: Morphological and Biomechanical Properties}

\author{
Pavel lablonskii, Serghei Cebotari, Igor Tudorache, \\ Sotirios Korossis, Andres Hilfiker, Axel Haverich
}

Medical Highschool Hannover, Hannover, Germany

Objective: Semilunar valve tissue engineering based on decellularized allograft valves has been successfully undertaken in order to create prostheses with reduced tissue immunogenicity and improved durability. In the present study we aimed to create tissue engineered mitral valve based on cell-free allograft valve scaffold. Methods: Ovine mitral valves were harvested at local slaughterhouse. The valves were decellularized using detergents. The effectiveness of decellularization was assessed by histological (haemotoxylin-eosin, Movat's Pentachrome) and immunofluorescent staining (for DNA and $\alpha-\mathrm{Gal}$ ). For assessment of tissue receptiveness to endothelial cells, the valve leaflets were reseeded with ovine endothelial progenitor cells (EPCs) in vitro. For biomechanical properties, uniaxial tensile testing was carried out to find the differences between native and decellularized leaflet tissue. Results: The complete decellularization was proved both by histological and immunofluorescent methods. According to software for immunofluorescence analysis, reduction in DNA and $\alpha-G a l$ was 99.99\% and 99.63\%, respectively. Uniaxial tensile test revealed not significant changes in ultimate tensile strain (native, $165.93 \pm 17.77 \%$ vs. decellularized, $216.32 \pm 30.07 \%$; $\mathrm{t}=0.33$ ), increased ultimate tensile stress (native, $1.29 \pm 0.36 \mathrm{MPa}$ vs. decellularized $2.5 \pm 0.42$ $\mathrm{MPa} ; \mathrm{t}=0.0015$ ) and Young's modulus (native, $1.98 \pm 0.77$ vs. decellularized, 2.19 $\pm 1.14 ; \mathrm{t}=0.01$ ). After EPCs seeding, immunohistochemistry revealed a monolayer of eNOS- and vWF-positive cells on the surface of the leaflet providing typical cobble-stone morphology. Conclusions: We have successfully created a decellularized atrioventricular homograft with reduced antigenicity, appropriate mechanical properties, and with a potential for autologous cell reseeding.

\section{The Medtronic Mosaic Aortic Valve, a 13-Year Single Center Experience}

Yorihiko Matsumoto, Tomoyuki Fujita, Hiroki Hata, Yusuke Shimahara, Shunsuke Sato, Junjiro Kobayashi

National Cerebral and Cardiovascular Center, Suita, Osaka, Japan

Background: This study aims to evaluate the long-term clinical performance of the Mosaic bioprosthesis (Medtronic, Minneapolis, MN) after aortic valve replacement. Methods: From 2000 to 2012, 207 patients (90 women; mean age, $74 \pm 8$ years, mean BSA, $1.48 \pm 0.25 \mathrm{~m}^{2}$ ) underwent aortic valve replacement with Mosaic bioprosthesis due to aortic stenosis in 154 patients and aortic regurgitation in 43. Prosthetic sizes were $19 \mathrm{~mm}$ in 103 patients, $21 \mathrm{~mm}$ in 53, $23 \mathrm{~mm}$ in 35, $25 \mathrm{~mm}$ in 13, and $27 \mathrm{~mm}$ in 3. Concomitant procedures included CABG in 52 patients, MVR in 17, MVP in 15 , Maze in 12, graft replacement in 12 , TAP in 8 , 
root enlargement in 2. Pre- and post-operative and follow-up echocardiographies were performed. Follow-up echocardiographies were performed in 124 patients (59\%) with the interval of $3.5 \pm 2.7$ years. Average follow up period was $3.5 \pm 3$ years (maximum, 12.4 years) and follow up rate was $93.7 \%$. Results: NYHA functional class was significantly $(\mathrm{p}<0.05)$ improved from $2.0 \pm 0.6$ to $1.1 \pm 0.3$. Early mortality was $3.4 \%$ and 5 - and 10 -year actuarial survival rate were $86 \%$ and $73.7 \%$. Valve related comorbidities were found in 3 (structural valve deterioration in 1 at 7.2-year and prosthetic valve endocarditis in 2 at 0.5 - and 3.1year), and 5- and 10-year freedom from valve related complications were $95.4 \%$ and $90.0 \%$. Echocardiography revealed that measured effective orifice area (post/follow up, $\mathrm{m}^{2}$ ) were $1.17 \pm 0.25 / 1.09 \pm 0.26$ in $19 \mathrm{~mm}, 1.29 \pm 0.19 / 1.20 \pm 0.26$ in $21 \mathrm{~mm}$, $1.39 \pm 0.24 / 1.24 \pm 0.33$ in $23 \mathrm{~mm}$ and $1.69 / 1.57 \pm 0.33$ in $25 \mathrm{~mm}$, and mean pressure gradient (post/follow up, $\mathrm{mm} \mathrm{Hg}$ ) were $19.4 \pm 6.0 / 21.9 \pm 8.4$ in $19 \mathrm{~mm}, 18.5 \pm 5.8 / 20.3 \pm 8.2$ in $21 \mathrm{~mm}$, $16.5 \pm 7.3 / 19.0 \pm 9.0$ in $23 \mathrm{~mm}, 13.2 \pm 2.9 / 11.3 \pm 4.7$ in $25 \mathrm{~mm}$. Left ventricular $(\mathrm{LV})$ mass was significantly $(\mathrm{p}<0.05)$ reduced postoperatively and at follow-up in each valve size with the regression rate of $74.6 \pm 18.8 \%$ in $19 \mathrm{~mm}, 75.5 \pm 30.2 \%$ in $21 \mathrm{~mm}, 68.1 \pm 30.5 \%$ in $23 \mathrm{~mm}, 55.9 \pm 12.9 \%$ in $25 \mathrm{~mm}$ and $49.2 \%$ in $27 \mathrm{~mm}$. There were no significant differences between each size in regression rate. Conclusion: The Mosaic aortic prosthesis showed excellent long-term durability with low incidence of valve related complications in elder patients group. Regardless of relatively high-pressure gradient, effective LV mass regression was obtained.

\section{Mid-Term Results of Aortic Root Reimplantation in Patients with Severe Aortic Regurgitation}

\section{Shunsuke Miyahara, Katsuhiro Yamanaka, Toshihito Sakamoto, Yoshikatsu Nomura, Takeshi Inoue, Masamichi Matsumori, Kenji Okada, Yutaka Okita}

Kobe University Graduate School of Medicine, Kobe, Japan

Objective: The aim of this study is to investigate the results of the valve-sparing root replacement with reimplantation technique in patients with severe aortic regurgitation (AR). Methods: From 2000 to 2013, 182 consecutive patients undergoing aortic root replacements were retrospectively reviewed. Clinical features and mid-term results of $88(48.4 \%)$ patients with preoperative severe $\mathrm{AR}$ (group S) were compared with those of less than severe AR (group C). More cusp prolapse (64.8\% in group $S$ and $22.3 \%$ in group $\mathrm{C}, \mathrm{p}<0.0001)$ and congenital bicuspid valve $(31.8 \%$ in group $\mathrm{S}$ and $8.5 \%$ in group $\mathrm{C}, \mathrm{p}<0.0001)$ was included in group $\mathrm{S}$. Less Marfan syndrome was included in group S (12.9\% in group S and $35.9 \%$ in group $\mathrm{C}, \mathrm{p}=0.0003)$. Patients in group $\mathrm{S}$ had deteriorated LV function; LVEF of group S and group C was $54.2 \pm 10.4 \%$ and $60.8 \pm 9.2 \%(\mathrm{p}=0.0001)$, and LVDd of group $\mathrm{S}$ and group $\mathrm{C}$ was $62.9 \pm 10.0 \mathrm{~mm}$ and $52.1 \pm 8.1 \mathrm{~mm}(\mathrm{p}<0.0001)$, respectively. Diameter of aorto-ventricular junction in group $\mathrm{S}$ was larger $(26.6 \pm 3.8$ $\mathrm{mm}$ in group $\mathrm{S}$ and $25.3 \pm 2.3 \mathrm{~mm}$ in group $\mathrm{C}, \mathrm{p}=0.0086$ ), but no significant difference was found in diameter of Valsalva sinus between groups $(\mathrm{p}=0.74)$. Results: Concomitant cusp repair was more frequently needed in group S (76.1\% in group S and $45.7 \%$ in group $\mathrm{C}, \mathrm{p}<0.0001)$. The overall survival at 5 and 10 years was $96.7 \pm 1.5 \%$ and $91.0 \pm 5.7 \%$, respectively, including 3 in-hospital deaths (1.6\%). Fourteen patients required reoperative aortic valve replacement or full root replacement: 11 for recurrent AR and 3 for endocarditis or graft infection. Freedom from reoperation at 5 year of group $S$ and group $C$ was $93.6 \pm 2.8 \%$ and $92.2 \pm 3.5 \%$, respectively $(\mathrm{p}=0.36)$. Freedom from moderate AR or greater at 5 year was $79.7 \pm 5.9 \%$ in group $\mathrm{S}$ and $89.0 \pm 3.8 \%$ in group $\mathrm{C}$, respectively $(\mathrm{p}=$ $0.19)$. Conclusions: Mid-term results of DVS root replacement performed in patients having severe AR preoperatively were satisfactory and comparable with those of moderate AR or less, despite complex cusp pathology and needs for concomitant cusp repair.

\section{Transcatheter Treatment of Failed Mitral Valve Repair and Deteriorated Bioprosteses}

\section{Gry Dahle, Arnt Fiane, Lars Aaberge, Bjørn Bendz, Jon T. Offstad, Jan Fredrik Bugge, Jan Hovdenes, Kjell Arne Rein}

Rikshospitalet, OUS, Oslo, Norway

Objective: Redo surgery in patients with failed valve repair or with deteriorated surgical bioprosteses is often challenging. In those pateients transcathater valve implantation may be an easier option and of benefit, especially if there are additional comorbidities and open coronary bypass artery grafting. This singel center presentation elucidate various, some still off labell, new transcatheter valve (THV) applications. Methods: Tvelve patients, five men, with mean age 72 years (44-83) were included. Mean logistic Euroscore was $26(10-48)$ and ejection fraction was mean 38 (15-45). Six patients had undergone additional CABG, two patients were in hemodialysis and three patients had prior pacemaker/ICD implanted. Two patients underwent transcatheter treatment following failed mital valve annuloplasty (one regurgitation/one stenosis), nine patients following deteriorated bioprostheses in mitral $(n=1)$, aortic $(n=6)$ tricuspidal $(n=1)$ and tricuspdal/pulmonal $(\mathrm{n}=1)$ position and one patient following paravalvular leak in an implanted catheter valve. The access was either transfemoral, transapical or transatrial. Edwards SAPIEN (Edwards Lifesciences, Irvine, CA) was used in all except for two patients who had one CoreValve (Medtronic inc. Minneapolis, $\mathrm{MN}$ ) and one Engager (Medtronic inc, Minneapolis, MN) implanted. Partial femoro-femoral bypass was used in two patients. Results: The procedural success was $92 \%$, one patient underwent open surgery due to paravalvualr leak. Three patients with the smalles valves had initially acceptable pressure gradients, upon three month follow up the gradients had incresed to $45 \mathrm{~mm} \mathrm{Hg}$. Two patients needed femoral artery reconstruction. Early mortality was $1 / 12(8 \%)$ and total mortality was $2 / 12(16 \%)$. Conclusions: Our results demonstrate the feasibility of transcatheter heart valve treatment for failed surgical valve repair and deteriorated bioprostheses in all positions, for stenosis as well as regurgitation. The appropriate accessroute and most suitable catheter valve must be choosen in each case. Maybe for the smallest valves $(<23 \mathrm{~mm})$ valve-in-valve should not be done, at least not with the Edwards Sapien XT. In the future this may play a significant role for the stategy in redo valve surgery. 
Table 1. (for Abstract of Gry Dahle)

\begin{tabular}{|c|c|c|c|c|c|c|c|c|c|}
\hline $\begin{array}{l}\text { Pt. } \\
\text { no. }\end{array}$ & $\begin{array}{l}\text { Valve } \\
\text { surgery }\end{array}$ & Other implants/surgery & Implant & $\begin{array}{l}\text { Size } \\
(\mathrm{mm})\end{array}$ & Failure & Approach & $\mathrm{CPB}$ & Transcatheter valve & $\begin{array}{l}\text { Size } \\
(\mathrm{mm})\end{array}$ \\
\hline 1 & MVP & TVP (MC3 Ring) & CE Physio & 34 & MR & TA & $\mathrm{Y}$ & E Sapien XT & 29 \\
\hline 2 & MVP & TVR (mech) PM CV, CABG & CE Physio & 28 & MS & TA & $\mathrm{Y}$ & E Sapien XT Two valves & $26 \times 2$ \\
\hline 3 & MVR & TVP (MC3 Ring) & $\mathrm{PE}$ & 27 & MS & TA & $\mathrm{N}$ & E Sapien XT & 29 \\
\hline 4 & AVR & CABG & $\mathrm{PE}$ & 25 & AS/AR & TA & $\mathrm{N}$ & E Sapien XT & 26 \\
\hline 5 & AVR & 0 & FS & 23 & AS/AR & $\mathrm{TF}$ & $\mathrm{N}$ & $\mathrm{CV}$ & 26 \\
\hline 6 & AVR & CABG & $\mathrm{CV}$ & 31 & PVL & TA & $\mathrm{N}$ & E Sapien XT & 29 \\
\hline \multirow[t]{2}{*}{7} & PVR & 0 & FS & 27 & PS & Tatr & $\mathrm{N}$ & E Sapien XT & 26 \\
\hline & TVR & & $\mathrm{HC}$ & 31 & TI/TS & & & & 29 \\
\hline 8 & AVR & CABG & $\mathrm{MO}$ & 23 & AS & TA & $\mathrm{N}$ & E Sapien XT & 23 \\
\hline 9 & AVR & CABG & $\mathrm{MO}$ & 23 & AS & $\mathrm{TF}$ & $\mathrm{N}$ & E Sapien XT & 23 \\
\hline 10 & TVR & 0 & $\mathrm{MO}$ & 29 & TS & Tatr & $\mathrm{N}$ & E Sapien XT & 29 \\
\hline 11 & AVR & 0 & CLOB & 25 & $\mathrm{AR}$ & TA & $\mathrm{N}$ & $\mathrm{EN}$ & 26 \\
\hline 12 & AVR & CABG & $\mathrm{MO}$ & 23 & AS/AI & TA & $\mathrm{N}$ & E Sapien XT & 23 \\
\hline
\end{tabular}

MVP = Mitral valve plasty; MVR = mitral valve replacement; TVP = tricuspidal plasty; $\mathrm{PM}=$ pacemaker; $\mathrm{CV}=\mathrm{Medtronic}$ Core Valve; CABG = coronary artery bypass grafting; MC3 = Edwards mc3 annuloplasty system; CE = Physio Carpenter Edwards Physioning; $\mathrm{PE}=$ Permont Edwards; FS = Medtronic Freestyle; $\mathrm{MO}=$ Medtronic Mosaic ultra; HC = Medtronic Hancock; CLOB = Cryolife O'Brien; $\mathrm{MR}=$ mitral regurgitation; $\mathrm{MS}=$ mitral stenosis; $\mathrm{AS}=$ aortic stenosis; $\mathrm{AR}=$ aortic regurgitation; $\mathrm{PVL}=\mathrm{paravalvular}$ leak, $\mathrm{PS}=$ pulmonal stenosis; $\mathrm{TI}=$ tricuspid regurgitation; $\mathrm{TS}=$ tricuspid stenosis; $\mathrm{CPB}=$ cardiopulmonary bypass; $\mathrm{Y}=$ yes; $\mathrm{N}=$ no; $\mathrm{E}$ Sapien $\mathrm{XT}=$ Edwards SAPIEN XT; EN = Medtronic Engager.

\section{Ross Procedure in Neonates and Infants: A European Multicenter Experience}

Aart Mookhoek ${ }^{1}$, Efstratios I. Charitos ${ }^{2}$, Mark G. Hazekamp ${ }^{3}$, Ad J.J.C. Bogers ${ }^{1}$, Jürgen Hörer ${ }^{4}$, Roland Hetzer ${ }^{5}$, Joerg S. Sachweh ${ }^{6}$, Arlindo Riso ${ }^{6}$, Ulrich Stierle ${ }^{2}$, Johanna J.M. Takkenberg ${ }^{1}$, Paul H. Schoof

${ }^{1}$ Erasmus University Medical Center, Rotterdam, Netherlands; ${ }^{2}$ University of Lübeck, Lübeck, Germany; ${ }^{3}$ Leiden University MedicalCenter, Leiden, Netherlands; ${ }^{4}$ German HeartCenter Munich, Munich, Germany; ${ }^{5}$ German HeartCenter Berlin, Berlin, Germany; ${ }^{6}$ University HeartCenter Hamburg, Hamburg, Germany; ${ }^{7}$ University Medical Center Utrecht, Utrecht, The Netherlands

Objective: Infants and neonates with severe left ventricular outflow tract obstruction may require replacement of the aortic root when more conservative treatment modalities are unsuccessful. In this retrospective multicenter cohort study, we present our experience with pulmonary autograft replacement of the aortic root in neonates and infants with a focus on late survival and freedom from graft reintervention. Methods: A total of 76 infants (age $<1$ year) from 6 centers in The Netherlands and Germany underwent the Ross procedure between 1990 and 2013. Results: Patients received the Ross (32\%) or Ross-Konno procedure (68\%) with a full, unsupported root. Median age at operation was 85 days (range 6-347 days). Hospital mortality $(\mathrm{N}=13,17 \%)$ was associated with neonatal age, pre-operative inotropic medication and circulatory arrest strategy at operation. Five patients (9\%) died during followup: 3 patients died of heart failure, 1 following balloon dilatation of a stenotic right ventricular outflow tract graft and 1 of unknown causes. Cumulative survival at 8 years was $75 \%$ (95\% CI $=63 \%-$
84\%) (figure 1). Only one patient required autograft reintervention after 75 days for aortic regurgitation due to a torn leaflet. At last follow-up, no patients presented with more than moderate (grade $\geq 3$ ) autograft regurgitation. Freedom from right ventricular outflow tract reintervention was $58 \%(95 \% \mathrm{CI}=40 \%-72 \%)$ at 8 years, with superior results in pulmonary homografts compared to aortic homografts or xenografts. Conclusions: The Ross procedure is an adequate surgical treatment for infants and neonates with critical aortic valve stenosis. At last follow-up, no signs of late autograft failure were observed. Late mortality was associated with chronic heart failure, which may be related to intrinsic abnormalities of the left ventricle or peri-operative ischemic damage.

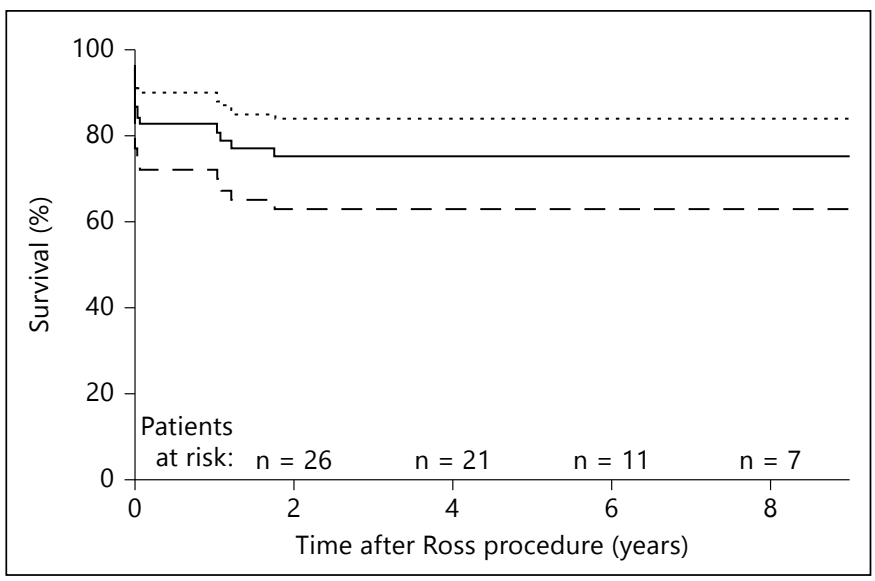

Fig. 1. Kaplan-Meier Curve of Overall Survival (for Abstract of Aart Mookhoek). 


\section{Durability of Mitral Valve Repair After Organic Mitral Insufficiency: 1-Year-Follow-Up Results in a Single Institutional Experience with Video-Assisted Minimally Invasive Approach}

\section{Udo Boeken, Jan-Philipp Minol, Tobias Weinreich, Hiroyuki Kamiya, P Akhyari, Artur Lichtenberg}

University Hospital, Duesseldorf, Germany

Objective: The feasibility of successful repair of Carpentier type II mitral insufficiency via MICS could be shown in different studies. However, due to the variety of underlying diseases, there is a great interest with regard to the durability of reconstructive surgery. Here we present our results with special regard to oneyear-folllow-up. Methods: For the prolaps of the anterior leaflet, implantation of neo-chordae (4/0 Gere-Tex) was the technique of choice. Resection of the leaflet was applied only for P2-prolaps with excessive tissue, and a sliding plastic was only done if inevitable due to calcification of the anulus or high risk for systolic anterior motion. Prolaps of the small P2-segment was treated with neo-chordae implantation. Prolaps of P1 and P3 was always treated with neo-chordae independent on the size of the segment. For the prolaps of both leaflets with excessive tissues (Barlow-syndrome), P2-resection and chordal transfer from P2 to A2 were performed. Anuloplasty ring was used in all cases. Results: We identified 156 patients with organic mitral valve regurgitation (Carpentier Typ II) from 307 patients underwent video-assisted minimal-invasive mitral valve surgery via right mini-thoracotomy. Using our standardized strategy, repair rate was $94 \%$ in all patients, $88 \%$ for the anterior prolaps, $95 \%$ for the posterior prolaps, $78 \%$ for the prolaps of both leaflet and $100 \%$ for Barlow-syndrome. A closed ring was used in 51 patients of 146 repairs (34.9\%). An open ring was inserted in $65.1 \%$. Conversion to full sternotomy was necessary in 3 patients $(2.0 \%)$ and early mortality was $1.3 \%$. At follow-up, survival was $94.2 \%$ in total. Freedom from reoperation was $87.2 \%$. Mean left ventricular ejection fraction decreased from $62.3 \pm 7.2 \%$ at hospital discharge to $61.6 \pm 7.0 \%(p>0.05)$ at follow in all patients. All follow-up results were comparable between the different underlying pathologies before mitral valve repair. Conclusions: The results clearly demonstrate that a very good shortterm outcome with excellent repair rates after mitral valve repair could be realized. Our follow-up data one year after surgery confirm these results with regard to patients' survival and functionality of mitral valve.

\section{Valve-Sparing Reoperations After Ross Procedure: A European Multicenter Experience}

\author{
Aart Mookhoek ${ }^{1}$, Laurent de Kerchove ${ }^{2}$, Gebrine El Khoury ${ }^{2}$, \\ Timo Weimar ${ }^{3}$, Giovanni Battista Luciani, \\ Alessandro Mazzucco ${ }^{4}$, Ad J.J.C. Bogers ${ }^{1}$, Diana Aicher ${ }^{5}$, \\ Hans-Joachim Schäfers ${ }^{5}$, Efstratios I. Charitos ${ }^{6}$, Ulrich Stierle ${ }^{6}$, \\ Johanna J.M. Takkenberg ${ }^{1}$
}

${ }^{1}$ Erasmus University Medical Center, Rotterdam, Netherlands; ${ }^{2}$ Cliniques Universitaires Saint-Luc, Brussels, Belgium; ${ }^{3}$ Sana Cardiac Surgery Stuttgart, Stuttgart, Germany; ${ }^{4}$ University of Verona, Verona, Italy; ${ }^{5}$ Saarland University Medical Center, Homburg, Germany; ${ }^{6}$ University of Lübeck, Lübeck, Germany

Objective: Autograft leaflet preservation at reoperation conserves the advantages of the Ross procedure, which include good hemodynamics and freedom from oral anticoagulation. However, repair is expected to result in lower durability compared to implantation of a mechanical valve prosthesis. In this retrospective multicenter cohort study, we present our experience with valve-sparing reoperations with a focus on long-term repair durability. Methods: A total of 85 patients from 6 centers in Europe underwent a valve-sparing reoperation following the Ross procedure between 1997 and 2013. Results: Valve-sparing repair was performed a median of 9.1 years (interquartile range 5.8-12.5 years, range 6 days to 20.7 years) after the Ross procedure. The initial Ross procedure, performed at a median age of 30.4 years (interquartile range $16.0-42.5$ years), was mostly performed with the full root technique $(85 \%)$. At the time of valve-sparing reoperation, severe aortic regurgitation (grade $\geq 3$ ) and cusp prolapse were present in $44 \%$ and $40 \%$ of patients, respectively. The reoperation included cusp repair in $44 \%$ of patients. In-hospital mortality was $1 \%$. During a median follow-up of 4.3 years, 3 more patients died of noncardiac causes resulting in a cumulative survival at 8 years of $89 \%$ (95\% CI: 65\%-97\%). Freedom from repaired autograft reintervention at 8 years was $76 \%$ (95\% CI: $57 \%-87 \%)$ with 15 patients requiring reintervention, mainly in the first 2 post-operative years (figure 1). Potential risk factors for autograft repair failure included isolated autograft regurgitation without root dilatation, young-

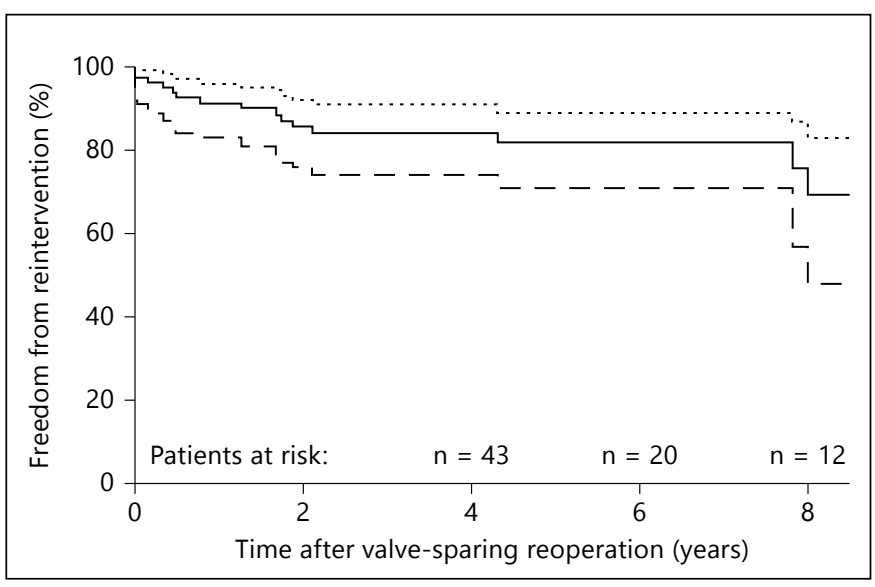

Fig. 1. Kaplan-Meier Curve of Autograft Repair Durability (for Abstract of Aart Mookhoek). 
er patient age at the time of repair and absence of autograft root replacement (isolated repair of autograft leaflets). In patients with a successful autograft repair, severe aortic regurgitation (grade $\geq 3$ ) was present in only $3 \%$ at last follow-up. Conclusions: Valve-sparing autograft reoperations carry a low operative risk, and in the majority of patients a durable result is achieved in the first postoperative decade. Repair durability in patients with isolated severe pulmonary autograft regurgitation is suboptimal, and requires careful preoperative weighing of surgical repair and replacement options.

\section{Distribution and Quantification of the Xenogeneic Alpha-Gal Epitope in Commercially Bioprosthetic Heart Valves}

\author{
Filippo Naso ${ }^{1}$, Michele Spina ${ }^{2}$, Laura lop ${ }^{1}$, Gino Gerosa ${ }^{1}$ \\ ${ }^{1}$ University Hospital of Padova, Padova, Italy; \\ ${ }^{2}$ University of Padova, Padova, Italy
}

Objective: For more than 30 years, glutaraldehyde (GLU) fixation has been considered as the standard chemical process able to ensure tissue biocompatibility, increased mechanical strength, sterilization, and safe storage of clinical bioprosthetic heart valves (BHVs). Nevertheless, the interplay of multiple factors leads to the replacement of GLU-treated BHVs after 8 to 10 years following the original implant. Important issue related to $\mathrm{BHVs}$ ' degeneration takes into account the efficiency of the GLU in ensuring biocompatibility of the treated xenogeneic tissue. BHVs express different epitopes proper of the species (bovine, porcine or equine), whose elimination or inactivation is mandatory to meet the requirements for a requisite improvement in clinical application. Unfortunately, GLU fixation procedure is not able to provide their complete masking, particularly the residual presence of alpha-Gal xenoantigen significantly increases the human anti-galactose titers, starting from day 10 following bioprosthetic heart valve (BHV) implantation and reaching a peak at around 3 months for IgM and IgG isotype, respectively. Methods: Seven different models of BHVs were tested. The number of epitopes was determined with reference to a standard alpha-Gal source by an ELISA test. The presence of xenoanti- gen was subsequently confirmed by immunofluorescence analysis. Results: Epic ${ }^{\mathrm{TM}}$ valve was the only model among those tested, in which the alpha-Gal antigen appeared to be completely shielded. Composite Trifecta ${ }^{\mathrm{TM}}$ valve exhibited conflicting results: cusps of bovine pericardial tissue were devoid of reactive alpha-Gal epitopes, while the stent cover strip of porcine pericardium still maintained $30 \%$ of active antigens originally present in native tissue. All other tested BHVs express an alpha-Gal amount not significantly different from that exhibited by porcine Mosaic valve (5.2 $\pm 0.6^{*} 10 \mathrm{e} 10$ each $10 \mathrm{mg}$ of tissue). Conclusions: For the first time, the quantitative evaluation of the alpha-Gal epitope in heart valve bioprostheses, already in clinical practice for about $40 \mathrm{yrs}$, was finally determined. Such quantification might provide indications of biocompatibility relevant for the selection of bioprosthetic devices and an increase in the confidence of the patient. It might become a major quality control tool in the production and redirection of future investigation in the quest for alpha-Gal-free long-lasting substitutes.

\section{Production of a New Generation of Alpha-Gal-Free Bioprosthetic Tissues: Preliminary Results}

\author{
Filippo Naso' ${ }^{1}$, Michele Spina ${ }^{2}$, Laura lop ${ }^{1}$, Paola Aguiari ${ }^{1}$, \\ Anabela C. Areias ${ }^{1}$, Catia M. Fidalgo ${ }^{1}$, Sugat R. Tuladhar ${ }^{1}$, \\ Gino Gerosa $^{1}$ \\ ${ }^{1}$ University Hospital of Padova, Padova, Italy; \\ ${ }^{2}$ University of Padova, Padova, Italy
}

Objective: Xenogeneic grafts are currently employed for the healing of diseased tissues. Strangely enough their use is permitted even in absence of any assessment of the elimination of xenogeneic cell material, as the alpha-Gal epitopes. The currently treatment with glutaraldehyde (GLU) is unable to grant a complete immuno-tolerance, reducing but not eliminating the immunogenicity particularly for the alpha-Gal epitope (the major hindrance for the success of xenotransplantation). Recently, our group has extensively reported studies focused on the evaluation of biocompatibility properties of xenogeneic material including commercially heart valve bioprostheses. In order to meet the criteria for the creation of free-alpha-Gal bioprosthetic material we are developing a new procedure able to remove completely such undesiderable
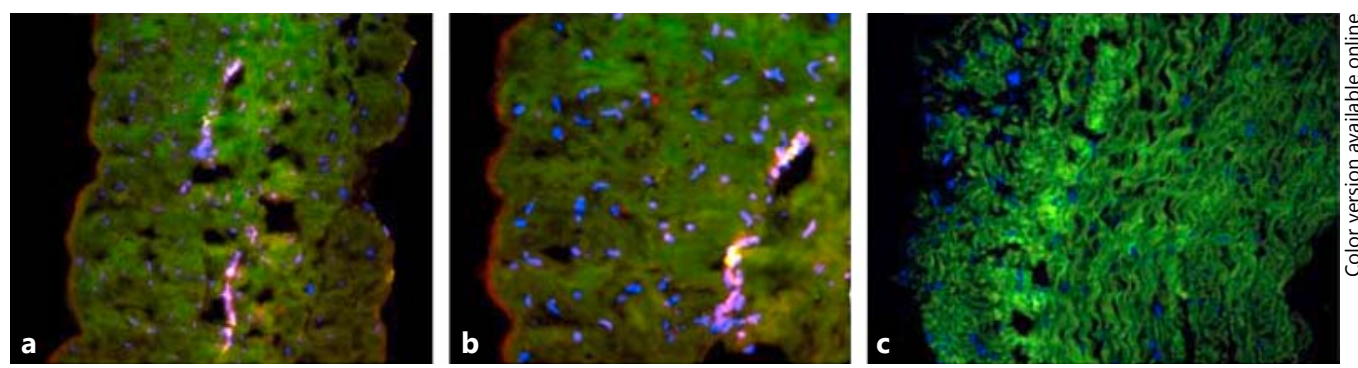

Alpha-Gal - Nucleic acid materials - Matrix autofluorescence

Fig. 1. Alpha-Gal epitope distribution in commercial equine pericardial patch before (a and b) and after (c) treatment aimed at the removal of this xenogeneic antigen, Magnification $20 \times(\mathbf{a}$ and c), $40 \times$ (b) (for Abstract of Gino Gerosa). 
epitope from GLU fixed tissues. Here we have been reported the first encouraging results obtained by the treatment of commercially available equine pericardial patch (EPP). Methods: Commercially available EPP (XAG-400, Edwards Lifesciences) are processed for the quantitative evaluation of the alpha-Gal epitope by a patented ELISA test before and after treatment with an extract of vegetable origin (patent pending). Immunofluorescence analysis was performed in order to confirm data. Monoclonal antibody M86 was adopted as specific alpha-Gal detector. Results: The EPP exhibited $9.7 \pm 4.9^{*} 10 \mathrm{e} 10$ alpha-Gal epitopes each $10 \mathrm{mg}$ of wet weight tissue. Such antigen residues are exposed and reactive notwithstanding the GLU treatment to which the tissue is subjected by the manufacturer. Such amount resulted completely removed after the treatment with our extract as further confirmed by the immunofluorescence investigation. Conclusions: First results showed that the removal of the alpha-Gal epitopes from GLU treated bioprosthetic tissue was possible without substantial modification in the manufacture process of biological devices. The elimination of the alpha-Gal residues will be able to ensure an improvement in the biocompatibility degree of the bioprosthetic tissue, avoiding the triggering of immunological/inflammatory chronic reaction and the onset of calcium enucleating site.

\section{Long Term Durability of a Sutureless Aortic Valve: In Vitro and In Vivo Evidences}

\section{Luc Behr, Nicolas Borenstein, François Laborde}

IMM RECHERCHE, Paris, France

Objective: The aim of this extensive in vitro and in vivo testing program was to provide evidence of the long term durability of the Perceval sutureless aortic valve (Sorin Group Italia Srl, Saluggia Italy). This collapsible valve is based on a highly innovative design leveraging on well proven biomaterials and processes, such as bovine pericardium treated with glutaraldehyde and homocysteic acid. Methods: In vitro evaluation consisted of bench tests: 1) accelerated leaflet wear testing ( $\mathrm{N}=52$ bioprosthesis, all sizes) simulating normotensive and hypertensive conditions, in regular and irregular anatomic setting; 2$)$ accelerated stent fatigue testing $(\mathrm{N}=$ 50 nitinol stents, all sizes) in hypertensive conditions; 3 ) histological evaluation of the pericardial valve leaflets after device collapsing. In vivo evaluation consisted of 5 months orthotopic implantation in juvenile sheep $(\mathrm{N}=6)$ with echographic, angiographic and histopathological assessment. Results: In vitro tests: Accelerated wear testing at 600 million cycles, i.e. 15 years of equivalent life, showed no Perceval valve failure and all samples had acceptable functional performance (EOA and regurgitation). All stents undergoing accelerated fatigue testing survived the 600 million cycles under hypertensive loading conditions. Histological evaluation of the valve leaflets after collapsing showed well preserved collagen structure. In vivo tests: echographic and angiographic assessment of peak $(11 \pm 10 \mathrm{~mm} \mathrm{Hg})$, mean $(6 \pm 5 \mathrm{~mm} \mathrm{Hg})$ transvalvular gradient, EOA $\left(1.3 \pm 0.3 \mathrm{~cm}^{2}\right)$ and regurgitation showed good haemodynamic performances. Explant examination showed proper integration by endothelial coverage of the valve components in contact with the aortic root. Neither alteration of the leaflets (i.e. tear, per- foration) nor of the stent (i.e. deformation, fracture) were observed. Semi-quantitative and quantitative inorganic $\mathrm{Ca}$ and $\mathrm{P}$ analysis demonstrated low levels of calcification. Conclusions: Long term durability represents the main concern for surgeons when implanting a newly developed cardiac valve prosthesis. The results we are reporting demonstrate the good performance of the Perceval sutureless valve in in vitrobench tests, and as well as in the in vivo study, where the sheep is recognized as the most severe model in predicting the risk of valve degeneration.

\section{Functional Mitral Valve Regurgitation and Coronary Artery Bypass Grafting in Patients with Ischemic Cardiomyopathy - Should We Operate Both Simultaneously?}

Jens Garbade, Rahel Kluttig, Laura Matz, Piroze Davierwala,
Martin Misfeld, Michael A. Borger, Friedrich-Wilhelm Mohr

Heart Surgery, Heart Center, University of Leipzig, Leipzig, Germany

Objective: The goal of this study was to analyze the outcomes of mitral valve (MV) surgery for functional mitral regurgitation (MR) in combination with coronary artery bypass grafting (CABG) in patients with severe ischemic cardiomyopathy. Methods: From 1999 to 2011, a total of 380 patients (288 male) with functional (MR) and systolic dysfunction (mean ejection fraction $24.8 \pm 6.2 \%$ ) underwent MV surgery combined with CABG. Mean logistic Euro Score was $19.1 \%$. Cardiogenic shock was present in $12.1 \%$ of patients and these patients were operated on as an emergency. Perioperative data was gathered prospectively and long-term follow up data retrospectively. Follow-up was $99.5 \%$ complete and was performed at a mean time span of 3.6 years postoperatively (range 0.01-11.5 years). Results: MV repair could be successfully performed in $88 \%$ of patients with implantation of a Carpentier-Edwards Physio Ring and or IMR Etilogix Ring in 57.6\% and $24.8 \%$ of patients, respectively. Of the 45 patients (12\%) undergoing MV replacement, 32 received a biological and 13 a mechanical valve. The mean number of coronary bypass grafts was $2.8 \pm 1$. Operation-, bypass- and cross clamp times were $326.1 \pm 77.5,134.8 \pm 47.0$ and $80.7 \pm 28.3$ minutes, respectively. Postoperatively, $20 \%$ of the patients needed an IABP and $2.6 \%$ required ECMO. The 30 -day mortality was $12.9 \%$. Long term mortality at 1,5 and 10 years was $25 \%, 41.1 \%$ and $51.3 \%$ respectively. Early echocardiographic follow-up revealed excellent MV function in most patients. MV-related reoperation rate was required in $7.4 \%$ during follow up. Redo MV replacement was performed in 10 patients $(2.6 \%)$ and redo MV repair in 2 patients $(0.5 \%)$ at a mean postoperative time of 591 and 55 days, respectively. Early coronary bypass revision was necessary in 4 patients (1.1\%). A further 13 patients required reoperation for other reasons including aortic valve replacement $(n=8)$, ventricular assist devices $(n=5)$ or heart transplantation $(n=2)$. Conclusions: MV and CABG surgery in patients with severe ischemic cardiomyopathy remains a challenging scenario. Despite the high riskrofile of these patients, adequate perioperative and longterm results can be achieved. 


\section{Long-Term Clinical Outcomes of the Toronto Stentless Porcine Valve: 15-Year Results}

\author{
Dai Une ${ }^{1}$, Reena Karkhanis ${ }^{1}$, Tiffany $\mathrm{Ng}^{1}$, Alexandra Linds ${ }^{1}$, \\ Tirone E. David" ${ }^{2}$ Bernard S. Goldman ${ }^{1}$ \\ ${ }^{1}$ Sunnybrook Health Sciences Centre, Toronto, ON, Canada; \\ ${ }^{2}$ Toronto General Hospital, Toronto, ON, Canada
}

Background: Stentless aortic bioprostheses have been reported to have excellent hemodynamics. This study examined the longterm durability of the Toronto Stentless Porcine Valve (TSPV) in the aortic position. Methods: We assessed long-term clinical outcomes of 515 patients with aortic valve replacement (AVR) with TSPV from 1987-2001 at 2 centers, excluding early ( $<30$ days) death. Valve function was longitudinally assessed by echocardiography. Median follow-up was 11.5 years (maximum 19.0 years). Survival and freedom from adverse events were calculated by Kaplan-Meier analysis. Independent predictors of events were assessed using Cox proportional hazards analyses. Results: Average age was $64.2 \pm 10.8$ years, and females were $34 \%$ (173/515). The Incidence of Patient-Prosthesis Mismatch $\left(\right.$ EOAI $\left.<0.85 \mathrm{~cm}^{2}\right)$ was low, $10 \%$. Overall survival was $90.9 \pm 1.3 \%, 75.8 \pm 2.0 \%$, and $57.7 \pm 3.2 \%$ at 5,10 and 15 years after surgery. Freedom from repeat AVR was $98.6 \pm 0.5 \%, 86.6 \pm 1.8 \%$ and $50.6 \pm 4.2 \%$ at 5,10 and 15 years. Over the follow-up duration, 116 patients (23\%) underwent repeat AVR: 97 for structural valve deterioration (SVD), 12 for endocarditis and 7 for other reasons. The 5-, 10- and 15-year freedom from SVD were $99.6 \pm 0.3 \%, 85.7 \pm 1.9 \%$ and $47.6 \pm 4.2 \%$. Independent predictors of mortality were age [odds ratio (OR) 1.5 per 10 years increase; $\mathrm{p}<0.001$ ], body surface area (BSA) (OR 1.6 per $0.5 \mathrm{~m}^{2}$ increase; $\left.\mathrm{p}<0.01\right)$, preoperative left ventricular dysfunction (defined as ejection fraction $<35 \%$ ) (OR 2.0; $\mathrm{p}=0.001$ ), diabetes mellitus (OR 1.9; $\mathrm{p}<0.001$ ) and chronic obstructive pulmonary disease (OR 1.8; $\mathrm{p}=0.03)$. Repeat AVR was associated with age (OR 0.5 per 10 years increase, $\mathrm{p}<0.001$ ), BSA (OR 2.0 per $0.5 \mathrm{~m}^{2}$ increase, $\mathrm{p}<0.001$ ) and diabetes mellitus (OR 0.3, $\mathrm{p}=0.049)$, while SVD was associated with age (OR 0.6, p < 0.001), BSA (OR 1.9, p = 0.001 ) and renal failure (OR 10.2, $\mathrm{p}=0.024)$. Conclusions: This study shows that the Toronto Stentless Porcine Aortic Valve is associated with excellent survival and durability during the first decade of follow-up. However, SVD increases after that, especially in younger patients with higher BSA and renal failure. These findings may assist with prosthesis selection. TSPV is no longer available but stentless valves may still have a role in AVR.

\section{Hemodynamic and Performance Measurements in Normal and Calcified Tricuspid and Bicuspid Aortic Valves}

\author{
Clara Seaman, Philippe Sucosky
}

University of Notre Dame, South Bend, IN, USA

Background: The bicuspid aortic valve (BAV) is a congenital defect occurring in 1-2\% of the population wherein the aortic valve forms with two leaflets instead of three. The BAV has a high- er incidence of valve stenosis, calcification, aortic dilation, dissection, and aneurysm. It has been hypothesized that the abnormal hemodynamics resulting from this valvular defect significantly contribute to valvular dysfunction and failure. Therefore, this study's goal is to present a comparative in vitro assessment of the flow and energy loss in a tricuspid aortic valve (TAV) and type-I BAV under normal and calcified states. Methods: Four valve models were considered in this study. The TAV model used a normal porcine valve and could be modified into a type-1 BAV model by suturing the left- and right-coronary leaflets. The calcified valve models were fabricated by injecting a solution of hydroxyapatite and agarose into the valve leaflets. Flow downstream of the four valve models, which were mounted in a physiologically relevant geometry and subjected to physiologic flow and pressure conditions, was characterized using particle-image velocimetry. Valve performance was evaluated using clinical metrics. Results: The normal TAV exhibited a circular orifice while the BAV orifice exhibited an elliptical shape. The calcified models featured irregular orifice shapes and demonstrated a reduction of up to $50 \%$ in orifice area relative to their non-calcified counterparts. Both healthy valve models exhibited an energy loss index greater than $1.35 \mathrm{~cm}^{2} / \mathrm{m}^{2}$, which is associated with healthy valve performance, whereas the diseased counterparts were below the healthy valve threshold. There was a $29 \%$ increase in the peak velocity between the normal and calcified TAV. A similar increase was observed between the normal and calcified BAV. The TAV had the lowest shear stress while the calcified BAV exhibited the highest level of shear stress. Conclusions: This study demonstrated that, while the TAV and BAV hemodynamics may be different, the BAV is not inherently diseased. However, when calcification was added to the TAV and $\mathrm{BAV}$, the valves behaved as clinically diseased valves and exhibited much higher velocities and stresses than their healthy counterparts.

\section{Systolic Anterior Motion After Mitral Valve Repair: Does It Have Long-Term Clinical Implications?}

\author{
Dan Spiegelstein, Rafael Kuperstein, Gilad Rotem, Michael Stein, \\ Shani Levin, Alexander Kogan, Leonid Sternik, Ehud Raanani \\ Chaim Sheba Medical Center, Ramat Gan, Israel
}

Objective: Systolic anterior motion (SAM) after mitral valve repair with significant mitral regurgitation (MR) is unacceptable, and requires immediate re-repair or valve replacement. Transient SAM immediately post repair is usually handled by hemodynamic maneuvers, such as afterload and preload increase and control of heart rate. In this study we investigated the late clinical significance of patients presenting with a post-operative tendency to SAM. Methods: Consecutive 549 patients with degenerative mitral valve (MV) disease underwent MV repair in our department. SAM was identifiedimmediately post-valve repair in 45 patients (8.2\%). Hemodynamic maneuvers such as preload, afterload increase and rate control were used. Of these 45 patients, 5 needed either a second pump-run and re-repair, or MV repair due to persistent SAM and significant MR. In the remaining 40 patients these maneuvers decreased SAM and reduced MR to less than mild, and these pa- 
tients formed the research group called SAM. At follow-up, we challenged those patients using stress-echo testing, and also compared late clinical and echocardiographic outcomes with all other patients without SAM. Results: There were 2 hospital deaths, one on each group $(\mathrm{p}=0.14)$. Mean follow-up was $54 \pm 28$ months and $31 \pm 26$ months, in SAM and non-SAM groups respectively $(\mathrm{p}<$ $0.05)$. Late stress echo demonstrated only one patient (2.5\%) in the SAM group with significant LVOT gradient $(>20 \mathrm{~mm} \mathrm{Hg}$ ), SAM and moderate MR. At 5 years, freedom from moderate or severe MR and freedom from NYHA FC III-IV were: $81 \%$ vs. $88 \%$ (p = $0.72)$ and $89 \%$ vs. $86 \%(\mathrm{p}=0.73)$, in the SAM and non-SAM groups respectively. Freedom from re-operation was $100 \%$ and $96 \%(\mathrm{p}=$ $0.4)$, and there were $0(0 \%)$ and $16(3 \%)$ late deaths $(\mathrm{p}=0.6)$, in the SAM and non-SAM groups respectively. All other late valve-related complications were similar between groups. Conclusions: Late post-operative stress echo revealed a non-significant incidence of SAM in patients with an immediate post-repair tendency to SAM. All other late clinical outcomes did not differ from those patients without transient SAM. Successful conservative management for intra-operative transient SAM is sufficient, demonstrating no evidence of late inferior outcome at rest and stress.

\section{Visualization of Aortic Valve Fenestration by Three Dimensional Transesophageal Echocardiography for Aortic Valve Repair}

Keitaro Mahara, Mitsuhiko Ota, Kanako Kishiki, Toshihiro Fukui, Shuichiro Takanashi

Sakakibara Heart Institute, Tokyo, Japan

Objective: Aortic valve repair requires a tailored surgical approach determined by the leaflet and aortic root characteristics. Fenestration of aortic valve is not an uncommon malformation, but sometimes cause aortic valve regurgitation. We thought to determine Three-dimensional (3D) transesophageal echocardiography (TEE) improves the identification of aortic valve fenestration that requires patch closure for aortic valve repair. Methods: We investigated consecutive 61 patients who underwent aortic valve plasty for aortic valve regurgitation in our institution from January 2012 to December 2013. Pre-operative 3D TEE was conducted within 3 months before surgery. Results: 9 patients had aortic valve fenestration that requires autologous pericardial patch closure. 7 of 9 patients was diagnosed as aortic valve regurgitation due to fenestration by pre-operative 3D TEE. Conclusions: 3D TEE could visualize aortic valve fenestration by careful observation of commissures. 3DTEE is essential in the operative assessment of aortic valve regurgitation because accurate determination of origin, lesion localization, and mechanisms is required to determine the surgical techniques.

\section{Conventional Aortic Valve Replacement: Convincing Results of a 5-Years-Single-Center Experience}

Branka Njezic, Frank Weinbrenner, Denise Marin, Albert Schuetz, Carole Hamilton, Stephen Hohe

Schoen Clinic Vogtareuth, Vogtareuth, Germany

Background: The treatment of patients requiring AVR is under discussion given the published results regarding TAVI which show a in-hospital-mortality of more than $15 \%$ and a high complication rate. In Germany all data regarding patient outcome is documented from every center by a system known as AQUA (Institute of applied quality promotion and research in Healthcare). The official results as of 2013 publisehd by AQUA show a mean in-hospital-mortality of $2.78 \%$ and xxx neurological events. We present our experience of 256 patients who underwent cAVR
Fig. 1. (for Abstract of Keitaro Mahara).

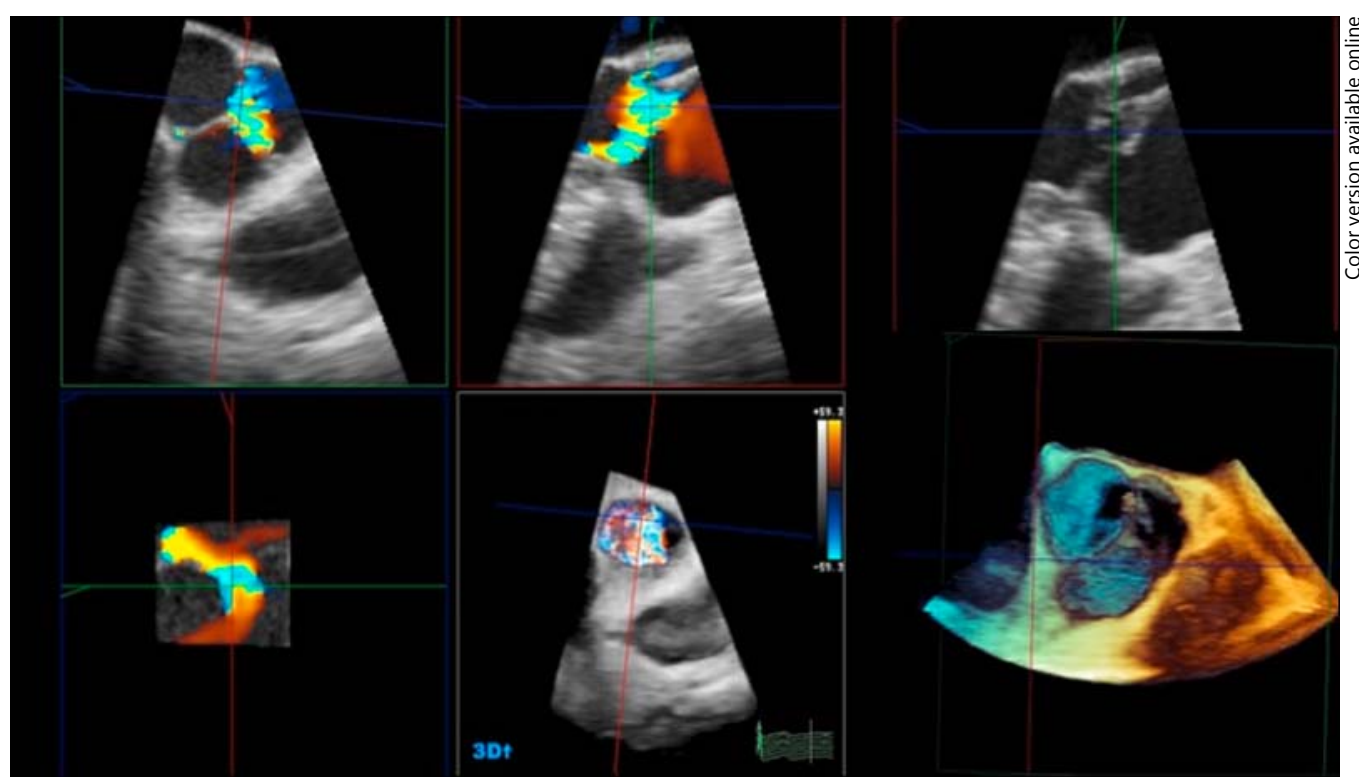


with $0 \%$ in-hospital-mortality and $0 \%$ neurological events. Methods: Over a 5 year period from January 2009 to December 2013 data was retrieved from the AQUA database to include patients who underwent elective AVR. Results: Conventional AVR was performed in 256 patients ranging an age from 30 to 89 years (mean age 68 years). Of the 256 patients 158 were males and 98 females, with 223 bioprosthesis and 33 mechanical valves. Conclusion: According to Aqua single AVR is one of the most common cardiac surgical procedures performed in Germany. These results show that cAVR is the best option for this group of patients. Proper indications for one method over the other should carefully be reconsidered.

\section{Value of Real Time 3D Transesophageal Echocardiography for Transcatheter Mitral Valved Stent Implantation in the Beating Heart}

Katharina Huenges ${ }^{1}$, Saskia Pokorny ${ }^{1}$, Telse Bähr ${ }^{1}$, Matthias Gegenwart ${ }^{1}$, Gunther Fischer ${ }^{2}$, Lucian Lozonschi ${ }^{3}$, Jochen Cremer ${ }^{1}$, Georg Lutter ${ }^{1}$

${ }^{1}$ University Hospital Schleswig-Holstein, Campus Kiel, Department of Cardiovascular Surgery, Kiel, Germany; ${ }^{2}$ University Hospital Schleswig-Holstein, Campus Kiel, Department of Pediatric Cardiology, Kiel, Germany; ${ }^{3}$ University of Wisconsin, School of Medicine and Public Health, Department of Cardiothoracic Surgery, Madison, WI, USA

Objective: In this study we report our experiences with realtime three-dimensional transesophageal echocardiography (RT$3 \mathrm{D}$-TEE) as adequate imaging technique during guidance and evaluation of mitral valved stent implantation in an experimental setting. Methods: Implantation of a mitral valved stent was performed in 28 pigs of the german landrace or Edelschwein
$(49.5 \pm 2.94 \mathrm{~kg})$ via a transapical approach in the beating heart. The implantation and deployment procedure were guided by $2 \mathrm{D}$ and RT-3D-TEE. A full two-dimensional (2D) and RT-3D-TEE evaluation based on a standard protocol was conducted before and $1 \mathrm{~h}$ after implantation. Focus of the assessment were the global heart function, the valved stent performance and position as well as possible paravalvular leakages (PVL) and central regurgitation (cMR). Results: The self-expanding valved stents were successfully implanted in 24 pigs at first attempt. Four pigs died during the procedure due to ventricular fibrillation. TEE evaluation showed a slightly decreasing ejection fraction $(63.54 \pm 5.13 \%$ to $56.68 \pm 8.83 \%)$ and E/A ratio (1.28 \pm 0.42 to $1.12 \pm 0.33)$, but still in physiological ranges. Mitral annular plane systolic excursion (MAPSE) as parameter for the longitudinal function slightly decreased after implantation $(1.2 \pm 0.1 \mathrm{~cm}$ to $1.0 \pm 0.1 \mathrm{~cm})$, but remained within a physiological range. Mildly increasing maximal velocities were observed over the mitral valve, the left ventricular outflow tract and the aortic valve (MV: $64.78 \pm 12.53 \mathrm{~cm} / \mathrm{s}$ to $88.30 \pm 21.68$, LVOT: $67.42 \pm 10.01 \mathrm{~cm} / \mathrm{s}$ to $97.29 \pm 43.23 \mathrm{~cm} / \mathrm{s}$, AV: $91.64 \pm 14.36 \mathrm{~cm} / \mathrm{s}$ to $102.90 \pm 25.41 \mathrm{~cm} / \mathrm{s})$. The gradients across the mitral valved stent remained low $(1.04 \pm 0.63 \mathrm{~mm} \mathrm{Hg}$ post implantation). RT-3D-TEE provided dependable spatial visualization of the left heart, mitral apparatus, delivery system and the valved stent. By the use of 3D-TEE orientation during deployment and positioning of the valved stent was notably facilitated. $3 \mathrm{D}$ showed particular benefit during evaluation of the stent performance and visualization of the stent movement and deformation. Conclusions: RT-3D-TEE provided accurate views and allowed assessment of regurgitations as well as the imaging of the stent, it is of explicit value as a guidance method during implantation. Therefore 3D-TEE highly contributed to the successful minimally invasive implantation of this novel valved-stent in the native mitral annulus.
Fig. 1. (for Abstract of Saskia Pokorny).
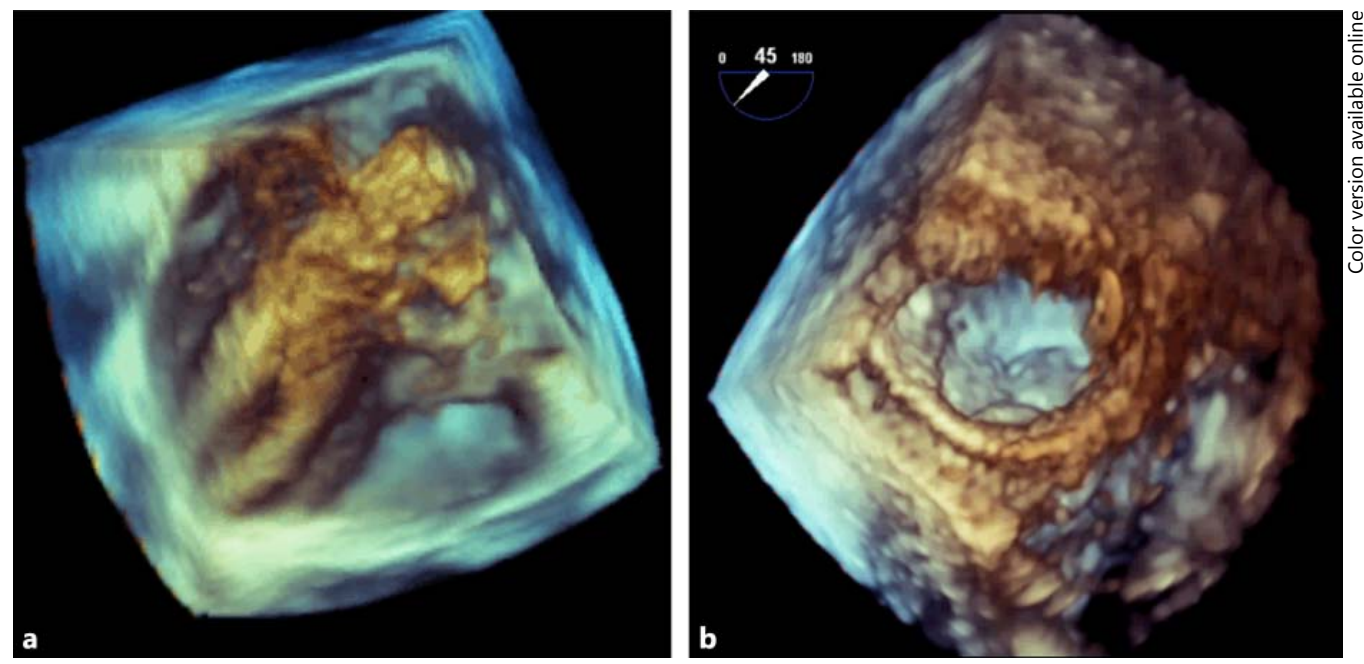


\section{The Handmade Composite Absorbable Tube for Pulmonary Artery Autograft Avoid PA Dilatation in the Ross Procedure}

\author{
Francesco Nappi, C. Spadaccio, S. Olivito, M. Chello, P. Fouret, \\ J.C. Chachquès, C. Acar
}

Centre Cardiologique du Nord, Paris, France

Objectives: The aim of the study was to develop a resorbable reinforcement of PA tailored to give structural support and to guide the process of wall structure modification for the preservation of graft viability. Methods: An experimental model of translocation of the pulmonary trunk as autograft in aortic position has been developed and performed under cardiopulmonary bypass in young lambs, with the PA left without reinforcement, reinforced with standard commercially available mesh, and reinforced with resorbable mesh of polyglactin and polydioxanone. The PA autograft diameter was measured using transoesophageal echography at $\mathrm{J} 0$ and at 6 months and compared to the distal aortic diameter. Pathological analysis of the PA autograft was performed at 6 months. Results were compared to those of a control group with no reinforcement $(\mathrm{n}=4)$ and to reinforcement with a non-absorbable mesh $(\mathrm{n}=3)$. Results: Animal weight was $27+5 \mathrm{~kg}$ at $\mathrm{J} 0$ and $55+10 \mathrm{~kg}$ at 6 months and the reference aortic diameter increased from $14+1 \mathrm{~mm}$ at J0 to $19+2 \mathrm{~mm}$ at 6 months. With no reinforcement, an instantaneous PA graft distension $(26+2 \mathrm{~mm})$ was noted followed by an aneurysmal formation at 6 months $(39+3 \mathrm{~mm})$. Pathological analysis revealed thinning of the vascular wall with one rupture. In all cases, the media presented with multiple disruptions. In the non-absorbable reinforcement group, the graft diameter remained unchanged and equal to the reference aortic diameter $(19+1 \mathrm{~mm}$ at $\mathrm{J} 0$ and $20+1 \mathrm{~mm}$ at 6 months), however histology showed in places an endoluminal migration of the mesh cutting through the PA autograft wall. Reinforcement with an absorbable material allowed maintaining the PA graft diameter close to the reference value $(18+3 \mathrm{~mm}$ at J0). At 6 months, mild PA dilation was noted in all 3 groups: polyglactin + polydioxanone suture: $29+4 \mathrm{~mm}$, 4-layer polyglactin: $28+2 \mathrm{~mm}$, and knitted polydioxanone: $27+1 \mathrm{~mm}$ transoesophageal echography revealed resorption of the mesh with no damage to the media. In the polydioxanone group, both the thickness and the ultrastructure of the PA wall resembled those of the aorta. Conclusion: In the paediatric Ross operation, reinforcement with a knitted polydioxanone mesh allowed subsequent growth while limitating pulmonary artery autograft dilatation.

\section{One Month Follow Up After Mitral Valved Stent Implantation in the Beating Heart: Reduced Paravalvular Leakages with New Prototype Design}

Saskia Pokorny ${ }^{1}$, Katharina Huenges ${ }^{1}$, Telse Bähr ${ }^{1}$,
Martin Marczynski-Bühlow ${ }^{1}$, Michael M. Morlock ${ }^{2}$,
Lucian Lozonschi $^{3}$, Jochen Cremer ${ }^{1}$, Georg Lutter'

'University Hospital Schleswig-Holstein, Campus Kiel, Department of Cardiovascular Surgery, Kiel, Germany; ${ }^{2} \mathrm{TUHH}$ Hamburg University of Technology, Institute of Biomechanics, Hamburg, Germany; ${ }^{3}$ University of Wisconsin, School of Medicine and Public Health, Department of Cardiothoracic Surgery, Madison, WI, USA

Objective: This study presents the results after transcatheter based transapical implantation of a mitral valved stent in the beating heart with follow up periods of one month or more. Methods: The self-expanding valved stent was implanted into the native mitral annulus of eight pigs via transapical approach in the beating heart. Hemodynamics, heart- and stent function were assessed following standardized protocols before implantation, $1 \mathrm{~h}$ after implantation, at one month and at outstanding health at monthly intervals using $2 \mathrm{D}$ and $3 \mathrm{D}$ TEE, hemodynamic measurements, ventriculography and cardiac CT. Results: Reliable stent stability and good position were achieved in all animals. Mean survival time was $44 \pm 22$ days (range 23 to 85 days). TEE evaluation showed slightly increasing transvalvular mean gradients across the mitral valved stent, the left ventricular outflow tract and the aortic valve $1 \mathrm{~h}$ after implantation $(\mathrm{p} \leq 0.043)$. However gradients were low and remained within a physiological ranges. The mitral annular plane systolic excursion slightly decreased after valved stent implantation ( $\mathrm{p}=0.012$ ), but was $\geq 1 \mathrm{~cm}$ at all times indicating good longi-

Table 1. Echocardiographic and hemodynamic results (for Abstract of Saskia Pokorny)

\begin{tabular}{llllll}
\hline Parameter & Pre & Post & p & 1 month & p \\
\hline EF (\%) & $65 \pm 3$ & $58 \pm 6$ & 0.012 & $52 \pm 15$ & 0.686 \\
Pmean MV (mm Hg) & $0.5 \pm 0.5$ & $1.2 \pm 0.7$ & 0.041 & $3.9 \pm 1.4$ & 0.080 \\
Pmean LVOT (mm Hg) & $1.0 \pm 0.3$ & $3.2 \pm 3.4$ & 0.043 & $2.1 \pm 2.4$ & 0.465 \\
Pmean AV (mm Hg) & $1.5 \pm 0.6$ & $3.1 \pm 1.9$ & 0.028 & $1.9 \pm 0.8$ & 0.197 \\
MAPSE (cm) & $1.2 \pm 0.1$ & $1.0 \pm 0.1$ & 0.012 & $1.1 \pm 0.2$ & 0.345 \\
E/A & $1.3 \pm 0.4$ & $1.1 \pm 0.1$ & 0.123 & $1.0 \pm 0.3$ & 0.686 \\
PCWP (mm Hg) & $7 \pm 3$ & $10 \pm 2$ & 0.050 & $17 \pm 3$ & 0.043 \\
PAP (mm Hg) & $14 \pm 4$ & $17 \pm 3$ & 0.122 & $25 \pm 8$ & 0.176 \\
\hline
\end{tabular}

Pmean = mean gradient over; $\mathrm{MV}=$ mitral valve / mitral valved stent; LVOT = left ventricular outflow tract; $\mathrm{AV}=$ aortic valve; MAPSE $=$ mitral annular plane systolic excursion; $\mathrm{PCWP}=$ pulmonary capillary wedge pressure; $\mathrm{PAP}=$ pulmonary artery pressure, ${ }^{*} \mathrm{p}-$ value of $\leq 0.05$ indicates a statistical significant difference compared to the pre-operative baseline value; $p=p$-value indicating a statistical significant difference, calculated based on a Wilcoxon test comparing the two successive evaluation results (pre-post, post-1 month). 
tudinal function. The E/A ratio was stable throughout the implantation procedure and follow up ( $\mathrm{p} \geq 0.123$ ). A physiological ejection fraction of greater than $50 \%$ was detected at all evaluation points. No paravalvular leakages (PVL) were detected in seven of eight animals directly after implantation. After one month mild PVL was detected in two and trace or less PVL in the remaining 6 animals. Evaluation of hemodynamic parameters revealed no relevant changes of the pulmonary artery pressure $(p \geq 0.080)$. The pulmonary capillary wedge pressure mildly increased $(\mathrm{p} \leq 0.05)$. Gross evaluation demonstrated correct stent position in all animals and good ingrowth within the native structures of the left atrium. Conclusions: Secure deployment and correct position of the valved stents was achieved in an off-pump procedure. Reliable stent stability, good alignment, low gradients, a normal longitudinal function and trace or less PVL after one month were achieved with this re-designed prototype in a follow up period of up to 85 days.

\section{The Analysis of Valvular Reoperations; 18 Years Experience}

\section{Nihat Cine, Mehmet Erdem Toker, Mehmet Tasar, Mehmet Dedemoglu, Mehmet Balkanay, Cevat Yakut}

Kartal Kosuyolu Heart Research and Training Hospital, Istanbul, Turkey

Objective: Number of valvular reoperations has increased over years due to various indications after the expanding pool of successful primary valvular operations. In this study, valvular reoperations performed in our hospital were evaluated retrospectively, and results were assessed. Methods: All valvular reoperations were included to the study from January 1993 - through December 2011. In that period, total of 693 patients underwent for valvular reoperation. The mean age was $45 \pm 13.5$ (13-82). Female patient comprised $64.5 \%$ (447), and male patient was $35.5 \%$ (246). The reasons for reoperations were reconstructive surgery in first operation (235 patients, 33.9\%), mechanical valv dysfunction (153 patients, $22.1 \%$ ), bioprosthetic dysfunction (136 patients, $19.6 \%$ ), periprosthetic leakage (87 patients, $12.6 \%$ ), new valve degeneration (45 patients, $6.5 \%$ ), and prosthetic valve endocarditis (37 patients, $5.3 \%)$. Mitral valves were replaced in 510 patients (73.6\%), aortic valves were replaced in 80 patients $(11.5 \%), 48$ patients (6.9\%) had aortic and mitral replacements, reconstruction of periprosthetic leakage was performed in 38 patients (5.5\%) for mitral position and 14 patients (2.0\%) for aortic position, isolated tricuspid valve reconstruction or replacement was performed in 3 patients $(0.4 \%) .605$ patients $(87.3 \%)$ had first reoperation, 81 patients $(11.7 \%)$ had second reoperation, and 7 patients $(1.0 \%)$ had third reoperation. Urgent and emergent cases comprised $12.7 \%$ (88) of all patients. Results: The in-hospital mortality rate was $15.7 \%$. Mortality rate was $12.9 \%$ for elective patients, and $35.2 \%$ for urgency and emergency patients. Mortality rates for underlying etiologies were; $32.4 \%$ for prosthetic valve endocarditis, $22.2 \%$ for mechanical valve dysfunction, $17.2 \%$ for periprosthetic leakage, $11.9 \%$ for reconstructive surgery in first operation, $11.1 \%$ for new valve degeneration, and $11.0 \%$ for bioprosthetic dysfunction. Mul- tivariate analysis demonstrated that total perfusion time $\geq 120$ minutes ( $\mathrm{p}<0.001$; OR 3.1, 95\% CI 1.9-5.0), urgency and emergency cases ( $\mathrm{p}<0.001$; OR 3.4, 95\% CI 2.0-5.9) and preoperative renal insufficiency ( $\mathrm{p}<0.001$; OR $3.7,95 \%$ CI 1.8-7.6) were independent risk factors for in-hospital mortality. Conclusions: Valvular reoperations can be carried out acceptable in-hospital mortality. Mortality rate has varied in different etiologies and patients clinical conditions. It is higher for the patients with prosthetic valve endocarditis, mechanical valve dysfunction and urgent/ emergent cases.

\section{Patient Experience with Current Clinical Decision Making in Prosthetic Aortic Valve Selection}

\author{
Nelleke M. Korteland ${ }^{1}$, Frans J. Bras ${ }^{2}$, Fabienne M.A. van Hout ${ }^{3}$, \\ Jolanda Kluin' ${ }^{2}$, Robert J.M. Klautz ${ }^{3}$, Ad J.J.C. Bogers ${ }^{1}$, \\ Johanna J.M. Takkenberg ${ }^{1}$ \\ ${ }^{1}$ Erasmus MC, Rotterdam, Netherlands; ${ }^{2}$ University Medical \\ Center Utrecht, Utrecht, Netherlands; ${ }^{3}$ Leiden University \\ Medical Center, Leiden, Netherlands
}

Background: To assess and compare among adult patients accepted for aortic valve replacement: (1) experience with current clinical decision making regarding valve selection, (2) preferences for shared decision making (SDM) and risk presentation, and (3) patient knowledge and numeracy. Methods: In a prospective multicenter cohort study patients scheduled for AVR were surveyed preoperatively and 3 months post-surgery. Results: Preoperatively 132 patients (89 males/43 females; mean age 67 years (range 2386)) responded. Ninety-nine percent of patients was aware that there are different types of aortic valve prostheses. Sixty-four percent of patients felt they had sufficient time to make a deliberate choice regarding the type of aortic valve prosthesis. Sixty-eight percent of patients wanted to be involved in decision-making, whereas $53 \%$ agreed that they actually were. Most patients $(68 \%)$ preferred scientific evidence presentation in a pie chart. Fifty-five percent of patients thought they had sufficient knowledge about the different types of aortic valve prostheses, and $68 \%$ of patients was actually able to answer three basic knowledge questions concerning prosthetic valves correctly. Regarding numeracy of patients: $38 \%$ of patients was able to answer three basic numeracy questions correctly. Sixty-four percent of patients experienced decisional conflict, and $28 \%$ to such extent that it made them feel unsure about the decision. Three months post-surgery 111 patients responded. Seventy-three percent of patients was satisfied with their aortic valve prosthesis, with no difference between patients with mechanical and bioprostheses. With regard to anxiety, patients with a bioprosthesis were more anxious about a possible re-operation than patients with a mechanical prosthesis ( $32 \%$ versus $11 \%$ respectively), while $26 \%$ of patients worried about oral anticoagulation treatment. Conclusions: In current clinical practice patients who undergo aortic valve replacement experience decisional conflict and suboptimal involvement in decision making, often have difficulties in understanding their choices and exhibit overt statistical illiteracy. Given the broad support for SDM among patients and the obvious need for understandable information, the introduction of a decision aid 
to support SDM in the setting of prosthetic aortic valve selection is expected to result in better informed and more actively involved patients, and less decisional conflict.

\section{Standardized Strategy for Repair of Barlow Syndrome via Minimally Invasive Approach}

Jan-Philipp Minol, Payam Akhyari, Udo Boeken, Tobias Weinreich, Hildegard Gramsch-Zabel, Hiroyuki Kamiya, Artur Lichtenberg

University Hospital, Duesseldorf, Germany

Objective: The Barlow syndrome defined as prolaps of the both leaflets with excessive tissue is sometimes difficult to repair. Here we present our technique for surgical treatment of Barlow syndrome with minimally invasive approach. Methods: At first, the whole mitral valve is inspected. Then, the P2-segment will be widely resected as triangular or quadrangular tissue after trimming and leaving the shortest chordae, normally the basal chordae at the P2-segment. This trimmed P2-segment will be transferred to the A2-segment. For this chordal transfer, the trimmed P2-segment will be sutured onto the top of the A2-segment on the ventricular side. In cases of quadrangular resection of the P2-segment, the annulus will be locally plicated. Then, the resected margins will be sutured in a running fashion. If the posterior annulus is severely calcified, the whole posterior leaflet will be detached from the annulus and annular decalcification will be done. Then, the P2-segment will be resected and chordal transfer will be done as described above and the posterior leaflet will be reconstructed as the sliding plasty including suturing the resected margins and re-attachment of the entire posterior leaflet to the annulus. Thereafter, an open annuloplasty prosthesis (in our hands Medtronic Future-Band) will be implanted. Results: Between August 2009 and August 2013, 22 patients (11 males, mean age 56 years old) with Barlow syndrome underwent video-assisted minimal-invasive mitral valve surgery via right mini-thoracotomy using this technique. No residual mitral valve regurgitation was seen in all patients. A slight systolic anterior motion (SAM) was seen in only one patient, which could be treated successfully with $\beta$-blocker medication. Conversion to full sternotomy was necessary in one patient $(4.5 \%)$ due to injury of the circumflex artery. Early mortality was $0 \%$. During follow-up, one patient died due to gastrointestinal bleeding. No patients needed reoperation for recurrence. Conclusions: This technique facilitates repair of Barlow syndrome with complex morphology and is durable in midterm follow-up.

\section{Feasibility of Procedures Apart from Mitral Valve Surgery via Right Lateral Minithoracotomy}

Jan-Philipp Minol, Payam Akhyari, Udo Boeken, Tobias Weinreich, Hildegard Gramsch-Zabel, Hiroyuki Kamiya, Artur Lichtenberg

University Hospital, Duesseldorf, Germany

Objective: Over the past decade right lateral mini-thoracotomy has been accepted as standard approach for isolated mitral valve surgery (MVS). However, further cardiac operations may also be realized via this access. We report the results of a 4-year experience with cardiac surgery via right lateral mini-thoracotomy (MICS). Methods: We retrospectively reviewed 361 cardiac operations via MICS with femoral cannulation between 8/2009 and $07 / 2013$. We investigated the short-term morbidity and mortality with regard to the surgical procedure and 1-year-follow-up data. It was our particular aim to analyze and to compare the results of non-mitral valve procedures. Results: Of the 361 patients undergoing MICS, 245 had isolated mitral valve (MV) operations (208 repairs, 37 replacements). Furthermore, there were 62 combined procedures with additional surgery of the tricuspid valve (TV), 25 isolated TV-procedures and 29 non-valvular procedures, such as resection of atrial tumors or closure of atrial septal defects. Overall in-hospital mortality (30 days) was $2.2 \%$ (8 of 361 ). In the individual groups, it was $0.4 \%$ ( 1 of 245 ), $3.7 \%$ (6 of 162 ) and $4 \%$ ( 1 of $25)$ in patients with isolated MVS, combined MV-procedures, and isolated TV-procedures, respectively. In patients with resection of tumors or ASD-closures there was no in-hospital death. Perioperative morbidity in all patients included reoperation for bleeding, neurologic complications (2.2\%) and disturbance of wound healing. The overall incidence of minor and major postoperative complications was significantly lower in patients with isolated MVS compared to all other groups. Length of ICU - and hospital-stay were $2.4 \pm 3.6$ days and $14.8 \pm 8.3$ days in all 361 patients (Isolated MVS: $1.7 \pm 1.6$ days and $13.9 \pm 6.2$ days; other groups: $3.1 \pm 4.1$ days and $15.9 \pm 3.1$ days, respectively). At follow-up, survival was $93.9 \%$ in total (Isolated MVS: $96.3 \%$; others: $88.7 \%$ ). Mean left ventricular ejection fraction was unchanged between hospital discharge and follow-up. $(59.7 \pm 10.4 \%$ vs. $59.4 \pm 9.1 \%$; $p>0.05)$. Conclusions: Right lateral mini-thoracotomy is a routinely used access for MVS. With our data we could also confirm excellent results with this access for a wide spectrum of further cardiac procedures.

\section{MicroRNA Profiling of Diseased Aortic Valves}

Sean Coffey ${ }^{1}$, Michael J.A. Williams ${ }^{2}$, Greg T. Jones ${ }^{2}$

1John Radcliffe Hospital, Oxford, United Kingdom;

${ }^{2}$ University of Otago, Dunedin, New Zealand

Objective: Given an increasing number of patients requiring aortic valve replacement and the current lack of medical therapies for calcific aortic valve disease (CAVD), there is an ongoing need for investigation into the underlying pathobiology of the disease. Studies examining diseased human valves are often hampered by the lack of an appropriate control group. MicroRNAs (miRNAs) are small non-coding RNAs that reduce transcription of mRNA, and, unlike mRNA, are stable in post-mortem tissue. Methods: We compared 15 aortic valves removed at aortic valve replacement for severe CAVD to 16 aortic valves obtained post-mortem using microRNA microarrays (Affymetrix GeneChip miRNA 2.0). Microarray results were validated in the same samples and an additional 26 samples, using quantitative polymerase chain reaction (qPCR). Control samples were sub-classified histologically by evidence of pre-clinical disease, in the form of macroscopic thickening, microcalcification, or atherosclerotic-like plaques. Results: Microarray analysis showed that 107 miRNAs were differentially 
expressed between severely diseased and control specimens, with miR-122-5p being the most down-regulated (adjusted p-value $3 \times 10^{-8}$ ) and miR-21-5p being the most up-regulated in diseased tissue (adjusted p-value $5 \times 10^{-6}$ ). Principal components analysis showed complete segregation of the two groups based on the miRNA profile. Validation using qPCR analysis confirmed differential expression of miR-122-5p, miR-21-5p, miR-221-3p, miR-30e-5p and miR-625-5p. MiR-200c-3p was down-regulated in microarrays but was significantly up-regulated in diseased valves on qPCR analysis. None of the validated CAVD associated miRNAs showed evidence of differential expression between control valve histological sub-groups. DIANA miRPath v2.0 analysis of the miRNAs measured using qPCR showed that glycosaminoglycan biosynthesis was the KEGG pathway showing most statistical significance for upregulated miRNAs (adjusted p-value $6.7 \times 10^{-14}$ ) while ubiquitin mediated proteolysis was the most statistically significant KEGG pathway affected by downregulated miRNAs (adjusted pvalue $6.1 \times 10^{-8}$ ). Conclusions: A network of miRNAs are associated with the pathobiology of advanced CAVD. The finding of fibrosis-related miR-21-5p being overexpressed was expected, while the suppression of miR-122-5p is likely related to its involvement in cholesterol metabolism. MiRNA-based therapies are currently in human trials for other disease processes, and future studies are needed to investigate the therapeutic potential of manipulation of miRNA levels in CAVD.

\section{In-Vitro Functionality Assessment of a New Tissue-Engineered Valve Substitute}

\author{
Catherine Tremblay ${ }^{1}$, Jean Ruel ${ }^{1}$, Véronique Laterreur ${ }^{1}$, \\ Karine Vallières ${ }^{2}$, Jean-Michel Bourget ${ }^{2}$, Maxime Y. Tondreau ${ }^{2}$, \\ Dan Lacroix², Lucie Germain'2, François A. Auger ${ }^{2}$ \\ ${ }^{1}$ Université Laval, Quebec City, QC, Canada; ${ }^{2}$ Centre LOEX, \\ Quebec City, QC, Canada
}

Background: Aortic valve replacement surgeries are usually performed using mechanical prostheses or tissue valves. While those prostheses have greatly evolved in the past decades, many serious drawbacks, such as lifelong anticoagulant therapies or accelerated calcification of the substitutes, often lower the life quality of the patients. Moreover, they still remain foreign materials to the body and are not able to remodel with their environment. The search for an entirely biological substitute that would eliminate those drawbacks is still relevant. Therefore, the purpose of this study is to develop a construction technique using the self-assembly method for a new biological heart valve substitute and to assess its functionality in a bioreactor. Methods: Dermal fibroblasts were cultured for 26 days in medium supplemented with ascorbic acid in order to create thin manipulable sheets. Cell sheets were stacked to create a thick plane tissue. Custom-made sets of templates were designed to help with the construction of the valve in its threedimensional configuration. The valve was then placed in a custom built bioreactor for three days to assess its functionality under a large variety of pulsed flow. Results: The shape of the $23 \mathrm{~mm}$ diameter tissue-engineered valve resembled that of a native aortic valve. The three leaflets presented a symmetrical configuration and adequately touched each other to create a tight and continuous contact surface. The valve was progressively exposed to pulsed flow with frequency ranging from $0.33 \mathrm{~Hz}$ to $1 \mathrm{~Hz}$. Flowrates were also modulated from very mild conditions to physiological-like flow rates. The valve maintained its shape throughout the whole experiment. Histological analysis of the valve at all steps of the construction technique presented a dense extracellular matrix and well-distributed cells. Conclusions: The self-assembly method seems a promising approach for the construction of a new heart valve substitute. The use of different sets of templates helped to establish a repeatable construction technique able to create a complex three-dimensional structure with only a plane tissue to begin with. In short-term tests, the new heart valve substitute was able to sustain a large variety of flow and pressure waves while maintaining its structural integrity.

\section{Impact of Concomitant Pulmonary Disease on Outcomes of Patients Undergoing Transcatheter Aortic Valve Replacement}

\author{
Nimesh Desai, Rohan Menon, Kelly Sutter, Lisa Walsh, \\ Daniel Choudhary, Wilson Szeto, Saif Anwaruddin, \\ Prashanth Vallabhajosyula, Howard Herrmann, Joseph Bavaria \\ University of Pennsylvania, Philadelphia, PA, USA
}

Objective: Transcatheter Aortic Valve Replacmeent (TAVR) is emerging as a viable treatment option for severe aortic stenosis (AS) in patients with significant co-morbidities. The purpose of this study is to determine the outcomes of patients with AS and COPD. Methods: Between November 2007 and June 2012, 266 patients underwent TAVR. All patients received a preoperative echocardiogram, heart catheterization, PFTs, and a 6-minute walk

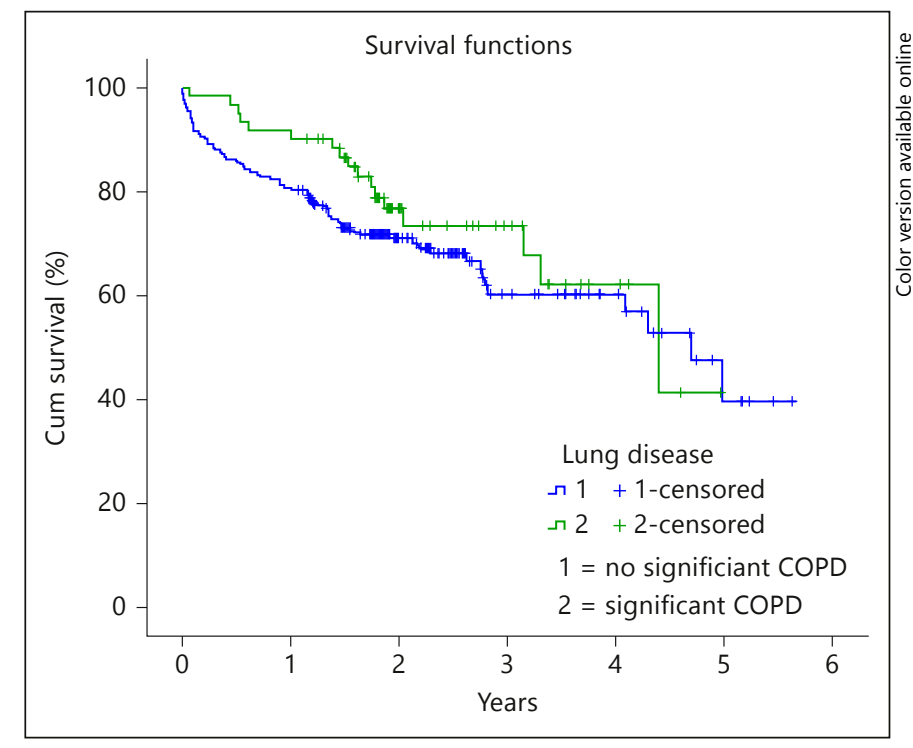

Fig. 1. (for Abstract of Nimesh Desai). 
test. 205 patients had no significant COPD (STS definition: non or mild pulmonary disease) and 61 patients had significant COPD (STS definition: moderate to severe pulmonary disease). Results: 1 -year data revealed $80 \%$ and $92 \%$ survival rates $(\mathrm{p}=0.28)$ of patients without significant COPD and those with significant COPD respectively. 1-year data also indicated that $9 \%$ of patients without COPD and $15 \%$ of patients with significant COPD were classified as NYHA III/IV ( $p=0.27$ ). From baseline to 1 -year, patients without significant COPD showed improvements on their 6-minute walk tests by 72.28 meters (54\%), while patients with significant COPD showed improvements by 50.50 meters $(42 \%)(p=0.045)$. 1 -year echocardiograms display improvements in both groups: ejection fraction (\%) from 57.5 to 61.2 and from 57.1 to 61.7 ( $\mathrm{p}=$ 0.37 ); mean gradients ( $\mathrm{mm} \mathrm{Hg}$ ) from 48.1 to 10.8 and 48.0 to 13.1 $(\mathrm{p}=0.27)$; and peak gradients $(\mathrm{mm} \mathrm{Hg})$ from 79.8 to 21.1 and 82.2 to 25.6 ( $\mathrm{p}=0.58)$, for patients without significant COPD and patients with significant COPD respectively. Conclusions: Early clinical and echocardiographic results demonstrate TAVR as a viable option for high-risk patients with severe aortic stenosis and COPD.

\section{Does Left Ventricular Hypertrophy Affect Outcomes in Patients Undergoing Transcatheter Aortic Valve Replacement?}

\author{
Nimesh Desai, Tyler Wallen, Saif Anwaruddin, \\ Prashanth Vallabhajosyula, Rohan Menon, Howard Herrmann, \\ Wilson Szeto, Joseph Bavaria
}

University of Pennsylvania, Philadelphia, PA, USA

Objective: Severe left ventricular hypertrophy (LVH) has a well documented severe negative impact on early mortality in patients undergoing surgical aortic valve replacement. This effect is particularly pronounced in patients with elevated relative wall thickness, an indicator of extreme hypertrophy with small ventricular cavity (suicide ventricle). It is not clear whether similar mortality risk is present in transcatheter aortic valve replacement (TAVR), which avoids issues regarding myocardial protection and hyperdynamic post-bypass physiology. The purpose of this study is to analyze the impact of $\mathrm{LVH}$ on patients undergoing TAVR. Methods: From 2008-2011, a retrospective review of patients undergoing tAVR was performed, comparing patients without severe LVH $(n=59)$ to those with severe LVH (group 2, n = 89). LVH was defined as a relative wall thickness (2xPosterior wall Thickness/ LVEDD) of greater than 0.5. Data was abstracted from a prospectively kept database with robust echocardiographic and clinical follow-up. Results: From 2008-2011, a retrospective review of patients undergoing tAVR was performed, comparing patients without severe LVH $(\mathrm{n}=59)$ to those with severe LVH (group 2, $\mathrm{n}=$ 89). LVH was defined as a relative wall thickness (2xPosterior wall Thickness/LVEDD) of greater than 0.5. Data was abstracted from a prospectively kept database with robust echocardiograhpic and clinical follow-up. Conclusions: Unlike surgical AVR patients, the presence of severe left ventricular hypertrophy in patients undergoing TAVR was not correlated with adverse outcomes at 1 year.

\section{Impact of Preoperative Anemia on Early and Late Outcomes After Surgical Aortic Valve Replacement}

Nimesh Desai, Rohan Menon, Wilson Szeto, Kanika Gupta, Prashanth Vallabhajosyula, Patrick Moeller, Michael Acker, Joseph Bavaria

University of Pennsylvania, Philadelphia, PA, USA

Objective: Red cell transfusion has been correlated with poor outcomes after open heart surgery. While operative indications for transfusion such as major bleeding may be difficult to modify, low preoperative hemoglobin may be addressed prior to surgery. The purpose of this investigation was to determine the impact of low preoperative hemoglobin on outcomes after surgical aortic valve replacement (AVR). Methods: From 2008-2012, we performed 1706 AVRs including 570 (32\%) with concomitant CABG. Acute endocarditis cases were excluded. Data was collected prospectively and analyzed using standard univariate, survival, logistic regression and Cox proportional hazard regression methodologies where appropriate. Results: Among 658 females, anemia (preop hemoglobin $\leq 11 \mathrm{~g} / \mathrm{dl}$ ) was present in 198 (30.1\%). Among 1048 males, anemia (preop hemoglobin $\leq 12 \mathrm{~g} / \mathrm{dl}$ ) was present in 347 (33.1\%). Multivariate risk factors for preop anemia included increasing patient age, diabetes, LV ejection fraction $(\mathrm{EF})<40 \%$, chronic renal insufficiency (CRI) and previous sternotomy. Preop anemia was strongly associated with periop blood transfusion (OR 2.8, 95\% CI 2.13.8). Other risk factors for periop blood transfusion included: Increasing patient age, concomitant $\mathrm{CABG}$, female gender, and previous sternotomy. In a fully adjusted model, preop anemia was associated with early mortality (OR 1.8, 95\% CI 1.2-3.1). Other early mortality risk factors included: Increasing patient age, concomitant CABG, female gender, $\mathrm{LV} \mathrm{EF}<40 \%$, CRI and previous sternotomy. Preop anemia was also highly associated with late mortality, $\log$ rank $\mathrm{p}<0.001$. Multivariate predictors of late mortality included; Preoperative anemia (HR 1.8, 95\% CI 1.3-2.5), increasing patient age, diabetes, LV EF $<40 \%$, and CRI. Conclusions: Preop-

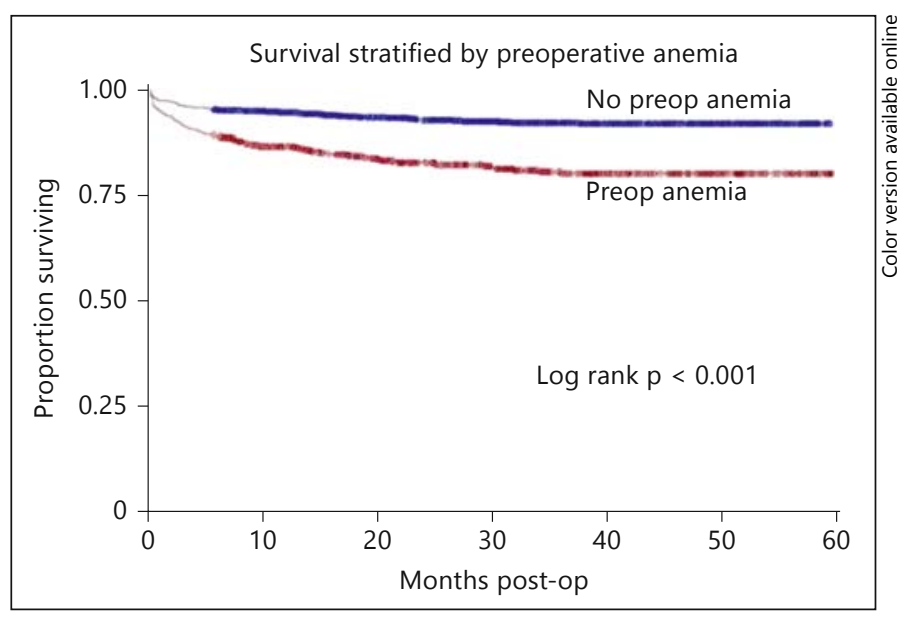

Fig. 1. Survival Stratified by Preoperative Anemia (for Abstract of Nimesh Desai). 
erative anemia is highly associated with mortality and this effect continues well beyond the perioperative period. Strategies aimed at modifying this risk factor warrant further investigation.

\section{Gender Differences Among Bicuspid Aortic Valve Patients Undergoing Aortic Valve Replacement: What Are the Implications?}

\section{Adin-Cristian Andrei, S. Chris Malaisrie, Jyothy John Puthumana, Vera Rigolin, Marla Mendelson, Zhi Li, Colleen Clennon, Robert O. Bonow, Patrick M. McCarthy}

Northwestern University, Chicago, IL, USA

Objective: Knowledge of gender differences, including outcomes and resource allocation, among bicuspid aortic valve (BAV) patients undergoing aortic valve replacement (AVR) is sparse. Methods: We analyzed 418 consecutive BAV patients $(317$ men and 101 women) that underwent AVR from 04/2004 to 04/2011 and had assessment of aortic diameter (AD). To reduce gender bias, we used propensity score (PS) matching to adjust for 22 factors, including age, comorbidities, other concomitant surgical procedures and maximal AD. Results: Women were significantly older than men $(61.9 \pm 13.6$ vs. $56.9 \pm 12.8$ years, $\mathrm{p}<0.001)$, had smaller AD ( $\mathrm{AD}<45 \mathrm{~mm}$ : $70 \%$ vs. $55 \%, \mathrm{p}=0.022)$ and similar follow-up $(4 \pm 2.2$ vs. $4 \pm 2.0$ years). For the total group, women had longer cross-clamp and bypass times $(\mathrm{p}<0.001)$, received smaller valve implants $(\mathrm{p}<$ 0.001 ), had longer intensive care unit (ICU) length of stay (LOS) $(\mathrm{p}=0.016)$, had more prolonged hospital LOS (third quartile (Q3) 7 vs. 6 days, $p=0.012)$, underwent more reoperations for non-cardiac reasons ( $7 \%$ vs. $2 \%, p=0.022)$ and required more postopera-

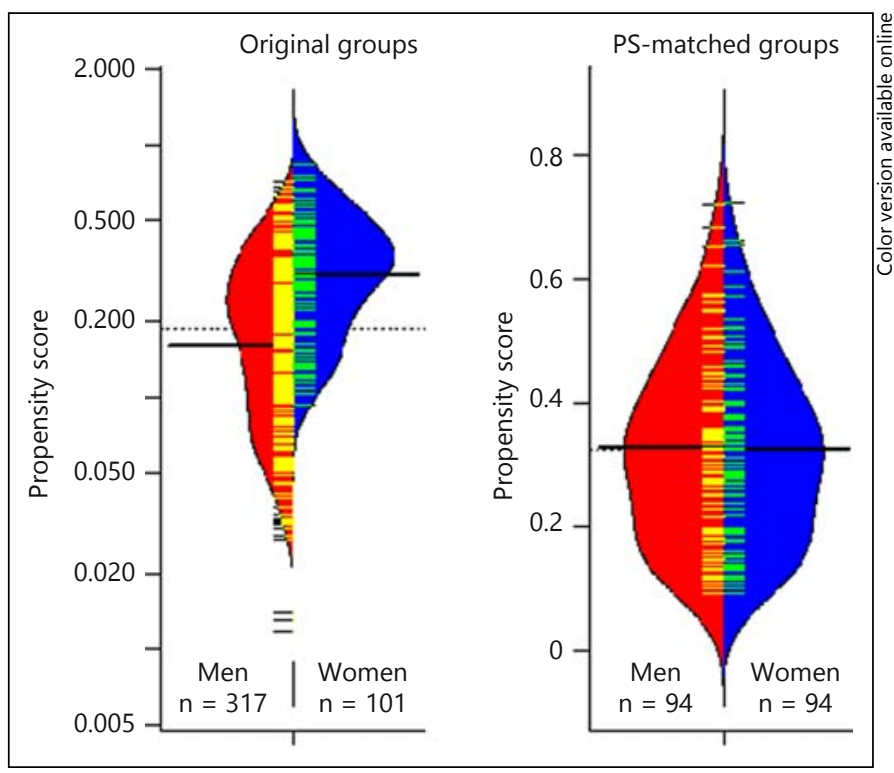

Fig. 1. Smoothed histograms of the propensity scores (PS) before and after PS-matching (for Abstract of Adin-Cristian Andrei). tive blood products ( $55 \%$ vs. $37 \%, \mathrm{p}<0.001)$. Operative, discharge and 30 -day mortality ( $1 \%$ men vs. $2 \%$ women, $\mathrm{p}=0.41)$ and overall survival $(90.6 \%$ men vs. $89.6 \%$ women at 5 years, $p=0.73)$ were similar. Figure 1 histograms indicate excellent PS-matching in the resulting 94 pairs. Women had longer ICU LOS (median 32.3 vs. 25.6 hours, $\mathrm{p}=0.005$ ), more prolonged hospital LOS (Q3 7 vs. 6 days, $\mathrm{p}=0.021)$, more reoperations for non-cardiac reasons $(6 \% \mathrm{vs}$. $0 \%, \mathrm{p}=0.013)$, more postoperative blood products ( $56 \%$ vs. $36 \%$, $\mathrm{p}=0.005)$. Women underwent atrial fibrillation surgical ablation less frequently ( $3 \%$ vs. $12 \%, \mathrm{p}=0.026)$. Operative, discharge and 30 -day mortality ( $1 \%$ men vs. $2 \%$ women, $\mathrm{p}=0.56$ ) and overall survival $(91.3 \%$ men vs. $91.5 \%$ women at 5 years, $\mathrm{p}=0.57)$ were similar. There were no aortic valve reinterventions during follow-up. Conclusions: Women had significantly longer ICU LOS, more prolonged hospital LOS, more reops for non-cardiac reasons and postoperative blood transfusions. Given these substantial differences, future resource-allocation and cost-effectiveness strategies require enhanced focus on gender differences.

\section{Prognosis of Patients with Filamin-A Related Mitral Valve Prolapse in a Large Family}

\section{Thierry Le Tourneau, Florence Kyndt, Simon Lecointe, Damien Duval, Antoine Rimbert, Jean Mérot, Jean Noel Trochu, Vincent Probst, Hervé Le Marec, Jean Jacques Schott}

Institut du Thorax, Nantes, France

Objective: Filamin-A-related mitral valve prolapse (MVP) is a $\mathrm{X}$ chromosome disease. We aimed to assess the outcome of patients with Filamine-A (FlnA) related mitral valve prolapse (MVP) in a large family. Methods: The study population comprised a large family carrying a P637Q FlnA mutation with a common ancestor born in the XVIII century. We identified 244 related members of the VI to IXth generations (birthdate 1925-2012). Phenotype was assessed by echocardiography in 105 patients including 49 with P637Q FlnA mutation. Overall survival and cardiac surgery rates were assessed in this large family from birth to 2013. Results: Phenotype assessment in 49 patients demonstrated that FlnA-MVP is both a developmental and a degenerative disorder involving mainly the mitral valve, but occasionally other valves. A P637Q mutation was ascertained in 61 patients $(F \ln A+)$ either by direct testing or by the identification of obligatory carriers (patient with descent carrying the mutation, or female children of a male carrier). Overall, FlnA status was determined in 212 relatives. Last follow-up was achieved in 2013 in the 212 relatives. Overall survival was not altered in Flna+ patients $(\mathrm{n}=61)$ compared with FlnA-relatives (at 70 year old, $80.3 \pm 9.6$ vs. $87.4 \pm 6.6 \%, p=0.22$ ) although there was a trend toward impaired survival in male patients $(57.3 \pm 17.0$ vs. $79.8 \pm 11.0, \mathrm{p}=0.10)$. Twelve patients were referred for cardiac surgery. Indications for cardiac surgery were mitral or aortic valve in 10 patients $(8 \mathrm{Fln} \mathrm{A}+), \mathrm{CABG}$ in 1 patient (Fln $\mathrm{A}-)$ and myxoma in 1 patient (FlnA-). Cardiac surgery rate was strongly increased $(30.7 \pm 10.9$ vs. $6.2 \pm 5.1, \mathrm{p}<0.0001)$ with a HR of 12.9 (95\% CI: 2.6 to 64.1$)$ particularly in male patients $(61.9 \pm 16.9$ vs. $11.1 \pm 10.5, \mathrm{p}<$ 0.0001 , HR 29.8). Conclusions: FlnA-MVP is a developmental and degenerative MV disease. In a large family with FlnA-MVP overall survival is not impaired although there is a trend toward a lower 
survival in male patients. However, the risk of cardiac surgery is strongly increased in FlnA+ male patients during life and appears higher in FlnA-MVP compared with Barlow disease. Valve surgery consisted not only of mitral valve surgery but also of aortic valve surgery for regurgitation or stenosis.

\section{Long-Term Results of the Ross Operation Versus Mechanical Aortic Valve Replacement: A Propensity-Score Matched Analysis}

\author{
Stefano Mastrobuoni Lurent De Kerchove, Silvia Solari, \\ Alain Poncelet, Parla Astarci, Robert Verhelst, \\ Philippe Noirhomme, Jean Rubay, Gebrine El Khoury
}

Cliniques Universitaires Saint-Luc, Université Catholique de Louvain, Bruxelles, Belgium

Objective: The pulmonary autograft (Ross Procedure) offers some advantages compared to bio-prostheses in young adult patients ( $<60$ years of age) who need aortic valve replacement (AVR), but it is unknown whether it is superior to mechanical AVR in this cohort. Aim of this study was to compare the long-term outcome of the Ross procedure compared to mechanical AVR in a propensity-score matched cohort of young adult patients. Methods: Between 1991 and 2013, 324 consecutive adult patients underwent elective aortic valve replacement with the Ross operation while 544 had mechanical AVR at Our Institution. Patients were matched by means of the Propensity Score (PS). Survival and long-term freedom from complications were compared in the matched cohort. Results: Ninety Ross patients were PS-matched to a mechanical AVR patient. Mean age was 51.3 years in the M-AVR and 45.5 years in the Ross group $(\mathrm{p}<0.01)$. Freedom from bleeding/thromboembolism at 10 years postoperatively was $80 \pm 5.4 \%$ and $92.7 \pm 3.2 \%$ in the M-AVR and Ross group respectively ( $\mathrm{p}=0.09$ ). Freedom from valve reoperation was $98.8 \pm 1.2 \%$ and $86.1 \pm 4.7 \%$ in the two groups respectively $(\mathrm{p}=0.01)$. Survival at 10 years was significantly better in the Ross group $(94.7 \pm 2.6 \%$ vs. $70.3 \pm 5.7 \%, \mathrm{p}=$ 0.001 ). Conclusions: The Ross operation has shown a higher risk of reoperation in the long-term compared to mechanical AVR and a similar risk of bleeding/thromboembolic complications. Nevertheless the long-term survival of Ross patients is significantly better than the M-AVR peers.

\section{Delayed Sternal Closure After Cardiac Surgery}

Siavash Saadat, Molly Schultheis, Joseph Romero, Anthony Azzolini, Viktor Dombrovskiy, Karen Odroniec, Peter Scholz, Anthony Lemaire, George Batsides, Leonard Y. Lee

Rutgers - Robert Wood Johnson Medical School, New Brunswick, NJ, USA

Background: Delayed sternal closure (DSC) has a reported incidence of $1.2 \%-4.2 \%$ in the adult cardiac surgical literature for indications including hemodynamic instability, marked myocar- dial edema, respiratory compromise, intractable bleeding, placement of assist devices, and persistent arrhythmias. The purpose of this study was to evaluate the incidence, survival, and morbidity of open chest management (OCM) patients who subsequently required DSC. Methods: All data were collected from the Robert Wood Johnson University Hospital Cardiac Surgery Database. A total of 1261 patients who underwent cardiac surgery from January 2012 through June 2013 were analyzed, evaluating postoperative morbidity and mortality, along with inciting conditions for the utilization of DSC. Chi-square and frequency analysis were performed using SAS 9.3 software (SAS Institute, NC). Results: A total of $41 / 1261(3.25 \%)$ cases resulted in DSC. Of the cases requiring DSC, 33/41 (80.5\%) were men and 8/41 (19.5\%) were women. Analysis revealed $7 / 41(17.1 \%)$ operations were coronary artery bypass graft (CABG), 2/41 (4.88\%) were mitral valve repairs (MVR), 2/41 (4.88\%) were aortic valve repair (AVR) combined with CABG, 2/41 (4.88\%) were MVR with CABG, and 28/41 (68.3\%) were other cardiac procedures, including placement of assist devices. There were no incidences of superficial sternal infection or mediastinitis in the DSC cohort, while infection occurred in $3 / 1220(0.25 \%)$ patients after conventional closure. Analysis of postoperative comorbidities revealed acceptable rates of postoperative stroke [2/41 (4.88\%)], atrial fibrillation [6/41 (14.6\%)], and renal failure [16/41 (39.0\%)]. Overall mortality was 14/41 (34.2\%). Reasons for planned DSC included bleeding $7 / 41$ (17.1\%), hemodynamic instability $11 / 41$ (26.8\%) and other cardiac abnormalities in $18 / 41(56.1 \%)$, while graft occlusion and valve dysfunction were not factors leading to the implementation of DSC. Conclusions: DSC is a technique that can be readily used in patients who require OCM for various reasons following cardiac surgery. DSC does not appear to increase the risk of infectious complications. Although postoperative complications such as stroke, atrial fibrillation, and renal failure, along with an acceptable mortality rate, is reflective of the patients' morbid condition requiring OCM, DSC can be carried out with a relatively low incidence of sternal complications after cardiac surgery.

\section{Long Term Advantages of Heart Valve Replacement Versus Valve Repair in Third World Countries}

\section{Imran Javed}

Fiji National University, Suva, Fiji

Objective: To compare advantages of heart valve replacement versus heart valve repair in poor countries of third world. Methods: It is a met-analysis comparing two major features of durability (Redo Surgery) and long term anti coagulation with warfarin with special focus on maintenance of therapeutic INR. Results: Mechanical Valve replacement is good solution with more durability in poor follow up areas but with more difficulty of maintenance of INR. Valve repair is better in terms of avoidance of long term warfarin need but all cases are not suitable for repair. In membranous valve replacement durability is limited as compared to mechanical one but limited need of anti coagulant therpay. Conclusions: Decision regarding choice of techniques should also take into account of local circumstances of healthcare delivery rather than just western oriented standard guidelines. 


\section{Myocardial Contractility Impacts Survival Prediction by Symptom Status After Aortic Valve Replacement}

\author{
Jeffrey Borer ${ }^{1}$, Phyllis Supino ${ }^{1}$, Edmund Herrold ${ }^{1}$, \\ Clare Hochreiter ${ }^{2}$, O Isom ${ }^{2}$, Karl Krieger ${ }^{2}$, Leonard Girardi ${ }^{2}$, \\ Nasimullah Khan ${ }^{1}$, Daniel Santarsieri ${ }^{1}$ \\ ${ }^{1}$ SUNY Downstate Medical Center, Brooklyn, NY, USA; \\ ${ }^{2}$ Weill Cornell Medical Center, New York, NY, USA
}

Background: Both preoperative symptoms and poor myocardial contractility individually predict mortality risk among patients (pts) with aortic regurgitation (AR) after aortic valve replacement (AVR). The combined influence of these characteristics on survival is unknown. Methods: We determined pre-AVR symptom status and myocardial contractility among 66 consecutively studied pts with severe AR and subsequent AVR (age 49 \pm 15 yrs at AVR, 86\% male). Symptom status was defined as New York Heart Association Functional Class (FC). Contractility was calculated as pre-AVR change $[\alpha]$ in LVEF from rest to exercise [ex], adjusted for $\alpha$ in end-systolic wall stress [ESS] from rest to ex [aEF$\alpha E S S]$, using combined echocardiographic and radionuclide cineangiographic data. We related a severe contractility deficit (previously defined, $\alpha E F-\alpha E S S \geq 17$ ) and symptom presence (FC 2-4) to late post-AVR survival. Results: During $15 \mathrm{yr}$ followup, 22 pts died (15 of cardiovascular cause). Cox model analysis demonstrated a statistical interaction between severe contractility and symptoms on post AVR death ( $\mathrm{p}=0.001$ [all causes], $\mathrm{p}=0.029$ [cardiovascular]). Log rank test comparisons of Kaplan-Meier curves showed that symptomatic patients with severe contractility had $\sim 3$ times more likely to die than pts without both descriptors (figure 1). Conclusions: In AR, the presence of a severe contractility deficit modulates the prognostic importance of pre-AVR symptoms. Pts with both characteristics are at increased risk for late postoperative death and should be closely monitored after AVR.

\section{Gender Differences and Early Outcome After Isolated Aortic Valve Surgery: A Nationwide Study}

\author{
Mostafa M. Mokhles ${ }^{1}$, Sabrina Siregar ${ }^{2}$, Michel Versteegh ${ }^{2}$, \\ Luc Noyez ${ }^{3}$, Bart van Putte ${ }^{4}$, Alexander B.A. Vonk ${ }^{5}$, \\ Jolien W. Roos-Hesselink ${ }^{1}$, Ad J.J.C. Bogers', \\ Johanna J.M. Takkenberg ${ }^{1}$
}

${ }^{1}$ Erasmus University Medical Center, Rotterdam, Netherlands; ${ }^{2}$ Leiden University Medical Center, Leiden, Netherlands;

${ }^{3}$ Radboud University Medical Center, Nijmegen, Netherlands; ${ }^{4}$ St Antonius Hospital, Nieuwegein, Netherlands; ${ }^{5}$ Free University Medical Center, Amsterdam, Netherlands

Objective: To compare women with men with respect to baseline characteristics and short-term outcome in a contemporary nationwide cohort of patients that underwent isolated AV surgery. Methods: All patients $(\mathrm{N}=8717 ; 56 \%$ males $(\mathrm{N}=4888))$ that underwent isolated AV surgery (replacement: $\mathrm{N}=8612,98.8 \%$; reconstruction: $\mathrm{N}=105,1.2 \%$ ) in the Netherlands between January 2007 and December 2011 were included in this study. Differences in patient and procedural characteristics, and in-hospital outcome were compared between male en female patients. Results: Female patients were generally older (mean age, 71 vs. 66 years, $\mathrm{p}<0.001$ ) and had higher logistic EuroSCORE (median score, 6.6 vs. $4.3, \mathrm{p}<$ $0.001)$. Male patients presented more often with extracardiac arteriopathy $(9.7 \%$ versus $6.7 \%$; $\mathrm{p}<0.001)$, prior cardiac surgery (12.3\% versus $6.9 \%$; $\mathrm{p}<0.001)$, renal disease $(2.3 \%$ versus $1.0 \%$; $<<$ $0.001)$, active endocarditis (5.9\% versus $1.7 \%$; $\mathrm{p}<0.001)$, critical preoperative state $(2.5 \%$ versus $1.5 \% ; \mathrm{p}=0.002)$, moderate $(15.5 \%$ versus $10.4 \%$; $\mathrm{p}<0.001)$ or poor $(4.2 \%$ versus $1.6 \%$; $<<0.001)$ left ventricular function and underwent more often emergent surgery (3.2\% versus $1.5 \%$; $<<0.001)$. Female patients underwent more often AV replacement (99.3\% versus $98.4 \%$; $<<0.001)$ and received more often stented $(68.7 \%$ versus $58.4 \%$; $<0.001)$ and stentless $(6.1 \%$ versus $3.4 \%$; $<0.001)$ bioprostheses. Male patients, on the other hand, received more often a mechanical prosthesis $(36.5 \%$ versus $24.4 \%$; $<<0.001)$. Female patients had comparable in-hospital mortality with male patients (OR 1.20, 95\% CI $0.90-1.61 ; p=0.220$ ). In males, the AUC for the logistic Euro-
Fig. 1. Impact of Preop Contractility Deficit on Post-AVR Survival Among Symptomatic Patients Only $(\mathrm{N}=40)$ (for Abstract of Phyllis Supino).
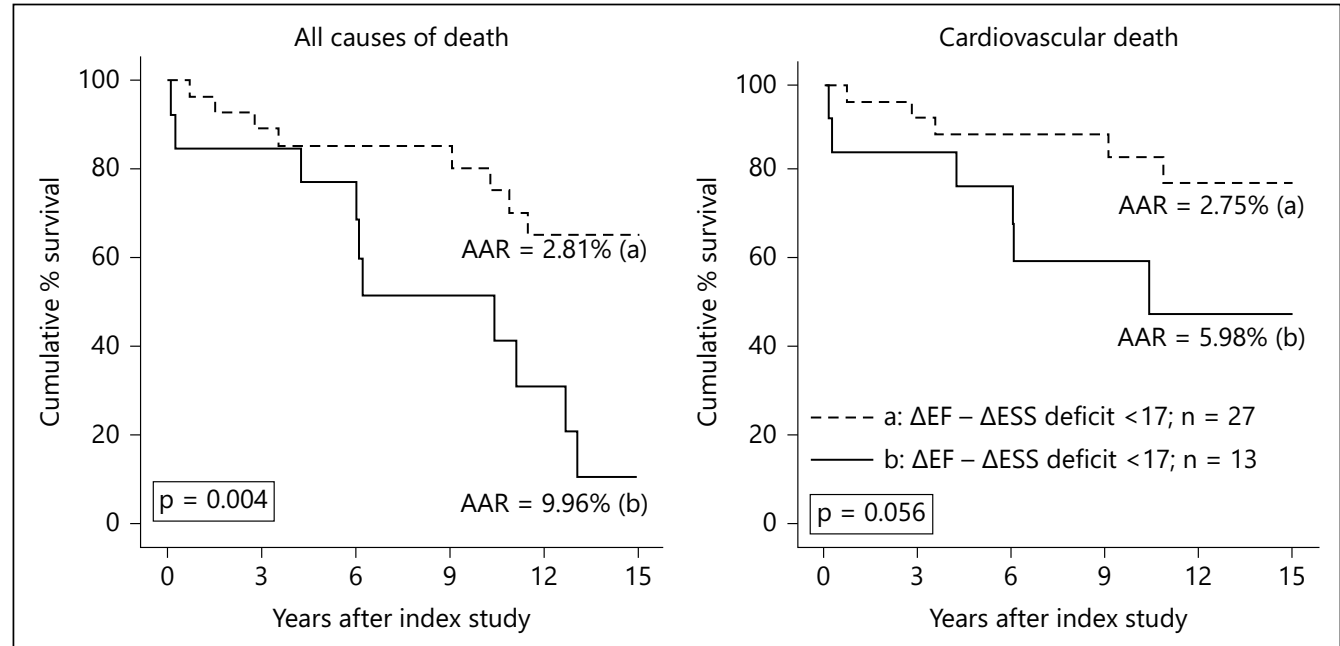
Table 1. Multivariate analyses of risk factors associated with in-hospital mortality in male and female patients (for Abstract of Mostafa M. Mokhles)

\begin{tabular}{|c|c|c|c|c|}
\hline \multirow[t]{2}{*}{ Characteristics } & \multicolumn{2}{|l|}{ Males } & \multicolumn{2}{|l|}{ Females } \\
\hline & OR (95\% CI) & p-value & OR $(95 \% \mathrm{CI})$ & p-value \\
\hline Age & $1.07(1.04-1.10)$ & $<0.001$ & $1.07(1.04-1.10)$ & $<0.001$ \\
\hline Chronic lung disease & $2.39(1.40-4.07)$ & 0.001 & & \\
\hline Extracardiac arteriopathy & & & $2.31(1.25-4.24)$ & 0.007 \\
\hline Neurological dysfunction & $2.63(1.04-6.66)$ & 0.042 & & \\
\hline Prior cardiac surgery & $2.72(1.57-4.73)$ & $<0.001$ & & \\
\hline Active endocarditis & $3.48(1.66-7.30)$ & 0.001 & $4.03(1.43-11.35)$ & 0.008 \\
\hline Critical preoperative state & $3.53(1.55-8.04)$ & 0.003 & & \\
\hline LV function & & & & \\
\hline Good & & & reference & \\
\hline Poor & & & $3.51(1.30-9.52)$ & 0.014 \\
\hline Recent myocardial infarction & $4.06(1.37-12.06)$ & 0.012 & $4.71(1.66-13.33)$ & 0.004 \\
\hline Pulmonary hypertension & $5.67(2.91-11.04)$ & $<0.001$ & $2.30(1.03-5.13)$ & 0.042 \\
\hline Prior valve surgery & & & $5.31(2.78-10.12)$ & $<0.001$ \\
\hline Prior aorta surgery & & & $3.29(1.24-8.74)$ & 0.017 \\
\hline Circ. arrest & $3.92(1.33-11.52)$ & 0.013 & & \\
\hline
\end{tabular}

SCORE was 0.82 (95\% CI 0.78-0.86) versus 0.75 (95\% CI 0.69 $0.80)$ in females. The calibration of the logistic EuroSCORE model resulted in $\mathrm{p}$-values of $\mathrm{p}=0.002$ and $\mathrm{p}=0.0033$ for males and females, respectively. Conclusions: Compared to males, female patients undergoing isolated aortic valve surgery are older, but have less EuroSCORE risk factors. The suboptimal performance of EuroSCORE in female patients warrants further exploration of gender-specific determinants of early mortality after isolated aortic valve surgery and calls for the development of gender specific risk stratification models for this group of patients.

\section{Bicuspid Aortic Valve Hemodynamics Contribute to Acute Remodeling in Porcine Ascending Aortas}

\section{Samantha Ratley, Kai Cao, Philippe Sucosky}

University of Notre Dame, South Bend, IN, USA

Objective: The type-I bicuspid aortic valve (BAV), which differs from the normal tricuspid aortic valve (TAV) by left-right coronary cusp fusion, is frequently associated with secondary aortopathies. While BAV aortic dilation has been linked to a genetic predisposition, hemodynamics has emerged as a potential alternate etiology. However, the link between BAV hemodynamics and aortic medial degeneration has not been established. The objective of this study was to compare the regional wall shear stresses (WSS) in the TAV and BAV ascending aorta (AA) and to isolate ex vivo their respective impact on aortic wall remodeling. Methods: The WSS environments generated in the convex region of a TAV and BAV AA were predicted through fluid-structure interaction (FSI) simulations in an aorta model subjected to both valvular flows. Remodeling of porcine aortic tissue exposed to TAV and BAV AA WSS for 48 hours in a cone-and-plate bioreactor was investigated via immunostaining, immunoblotting, zymography, and gene analysis. Results: FSI simulations revealed the existence of larger and more unidirectional WSS in the BAV than in the TAV AA convexity. Exposure of normal aortic tissue to BAV AA WSS resulted in increased MMP-2 and MMP-9 protein expressions and MMP-2 activity but similar fibrillin-1 content and microfibril organization relative to the TAV AA WSS treatment. Gene analysis on the expression levels of the ratio of matrix metalloproteinases (MMPs) to their inhibitors, TIMPs, showed that the BAV AA convexity has a higher ratio of MMP-9/TIMP-1. Conclusions: This study confirms the sensitivity of aortic tissue to WSS abnormalities and demonstrates the susceptibility of BAV hemodynamic stresses to focally mediate aortic medial degradation. The increased ratio of MMP-9 to TIMP-1 indicates accelerated remodeling events at 48 hours as compared to TAV AA WSS conditioned tissue and fresh tissue. The results provide compelling support to the dominant role of hemodynamics in BAV secondary aortopathy.

\section{Beating Heart Valve Surgery Post Myocardial Revascularization with Patent Grafts}

\section{Paolo Pepino, Salvatore Giordano, Piermario Oliviero, Germano Coronella, Raffaela Provenzano, Giuseppe Pescatore, Antonio Contaldo, Antonella Luciano, Mario Monaco}

Presidio Ospedaliero Pineta Grande, Castel Volturno, Caserta, Italy

Objective: Valve surgery in patients with previous CABG and patent arterials conducts is a challenging procedure. To avoid any risk of injury the grafts, we performed valve surgery with the beating heart: 4 aortic valve replacement (AVR); 1 ascending aorta replacement and 2 mitral valve replacement (MVR). Methods: From 
September 2010 up to May 2013 seven patients (four male and three female, aged $67.66 \pm 10.85$, pre op LVEF $47.85 \pm 6.46$ ) with a story of previous CABG (three patients using left internal mammary artery + saphenous vein; four patients using LIMA + radial artery; one patient using also the right internal mammary artery, number of by pass 2.85 \pm 0.63 ) were admitted in our department for valvular or ascending aorta disease. All patients had previous coronarography with patent arterial conduit in six patients, while in one patient an occluded LIMA graft on the LAD and a patent radial artery graft from the LIMA on the first and second obtuse marginal were observed. Reoperations were performed on beating heart. Arterial conducts were not isolated to avoid possible injury and the procedures were performed cross-clamping the aorta, on pump, perfusing the coronary trough the patents graft and with a retrograde perfusion with a cannula in the coronary sinus. Results: The aortic cross clamp time was $81.8 \pm 20.1$. Two patients had AVR with biological valve, two patients had AVR with mechanical valve, one patient had replacement of the ascending aorta with a dacron graft and two patients had MVR with biologic valve. No major complications were observed. No in hospital mortality was observed and all patients were discharged in $6.5 \pm 0.9$ days post op, showing a LVEF of 49.28 \pm 5.62 . Conclusion: Beating heart surgery is a safe procedure in patients who received a previous CABG. Maintaining coronary perfusion through the patents arterial conducts and through the retrograde perfusion will protect the heart without any ischemia.

\section{Off Pump Aortoplasty for Selected Ascending Aortic Aneurysm}

\section{Paolo Pepino Germano Coronella, Piermario Oliviero, Raffaela Provenzano, Salvatore Giordano, Agostino La Marca, Francesco Petteruti, Mario Monaco}

Presidio Ospedaliero Pineta Grande, Castel Volturno, Caserta, Italy

Background: Sixty percent of thoracic aortic aneurism involve the aortic root or ascending aorta. The optimal timing of surgical repair remains uncertain despite most surgeons prefer to treat the aneurysm at a diameter of $\geq 5.5 \mathrm{~cm}$. This paper describes our off pump option for the treatment of isolated asending aorta aneurism with a diameter between 5.0 and $5.5 \mathrm{~cm}$. Methods: Four patients with a story of ascending aorta aneurysm were selected from March 2013 to September 2013. Trans thoracic echocardiography, angio CT scan and a coronary angiography were performed during the pre op evaluation. The surgical procedure was performed on beating heart and off pump using with a low dose of heparin (5000 UI). A side clamp was used to plicate longitudinally the ascending aorta at the level of maximum dilatation from the sino tubular junction up to the troncus anonymous with controlled hypotension (70 $\mathrm{mm} \mathrm{Hg}$ ). The plication was performed with a double layer of $3 / 0$ Prolene suture reinforced with two strips of Teflon along the plication. Furthermore, a $32 \mathrm{~mm}$ Dacron tube, opened longitudinally, was wrapped around the ascending aorta and sutured to the plication with a double $3 / 0$ prolene suture. Results: All patients were estubated in an hour after the operation. No major complications were observed. A post op echocardiography and an angio CT scan were performed at the discharge showing a reduction of the ascending aorta aneurysm with a maximum diameter of $3.5 \mathrm{~cm}$. Conclusion: The procedure previously described is easy and it is done without cardiopulmonary bypass that according to the Laplace low (the wall tension is the result of the pressure for radius) reducing the radius of the aorta, allows to reduce the wall tension. Furthermore the bending of the ascending aorta reduces the possibility of rupture of the aorta. This is a preliminary experience that despite initially results are encouraging (CT scan after six month doesn't show any changes) must be controlled with a mid term follow up.

\section{Functional and Prognostic Implications of NT-proBNP and Exercise Stress Echocardiography in Asymptomatic Patients with at Least Moderate Mitral Regurgitation and Preserved Left Ventricular Systolic Function}

Sung-Ji Park' , Eun Jeong Cho', Sung-A Chang ', Sang-Chol Lee', Dong-Ju Choi ${ }^{2}$, Seung Woo Park ${ }^{1}$

${ }^{1}$ Samsung Medical Center, Seoul, Republic of Korea;

${ }^{2}$ Seoul National University Bundang Hospital, Seongnam,

Republic of Korea

Objective: The management of patients with asymptomatic degenerative MR remains controversial. Also, early symptoms can be difficult to detect clinically and imply LV dysfunction can develop without recognized symptoms. Exercise is recommended in patients who have discordance between the severity of MR and symptoms and can be used to identify LV dysfunction in patients with chronic MR. And NT-proBNP was the best marker for monitoring left ventricular dysfunction. So, in asymptomatic MR, exercise echocardiography and NT-proBNP provide the clinician with important incremental clinical determinants that can contribute importantly to identify a subset of patients at higher risk, who may benefit from elective MV surgery. Methods: A symptomlimited treadmill exercise testing using the modified Bruce protocol was performed in 114 asymptomatic patients ( $55.4 \pm 14.6 \mathrm{yrs})$ with at least moderate $\mathrm{MR}(\mathrm{ERO}>20 \mathrm{~mm}, \mathrm{RV}>30 \mathrm{ml})$ and preserved LV function (EF $>50 \%$ ). Clinical symptoms, vital signs, ECG, and Doppler hemodynamics obtained during and/or immediately after exercise. Post-exercise EF increment of $>4 \%$ was defined as $\mathrm{CR}+$ and those without, as CR-. Results: There were significant differences between the above the NT-proBNP of 400 group and the under the NT-proBNP of 400 group including age, LVIDd, LVIDs, LAVI, LVMI, rest RVSP, Duke's score and CR. The above the NT-proBNP of 400 group showed higher MV operation than the under the NT-proBNP of 400 group. In Logistic regression analysis, the independent predictors of MV repair or MVP were NT-proBNP 400 ( $\mathrm{p}=0.023)$. In sequential Cox models, a model based on clinical data and resting echocardiography parameter $\left(\chi^{2}=20.456\right)$ was improved by stress echocardiography paramerer $\left(\chi^{2}=31.026 ; \mathrm{p}=0.005\right)$ and NT-proBNP $\left(\chi^{2}=37.607\right.$; $\mathrm{p}=0.010)$. Conclusions: In asymptomatic patients with at least moderate degenerative MR and preserved LV systolic function, exercise stress echocardiography and NT-proBNP provide important incremental clinical determinants. 


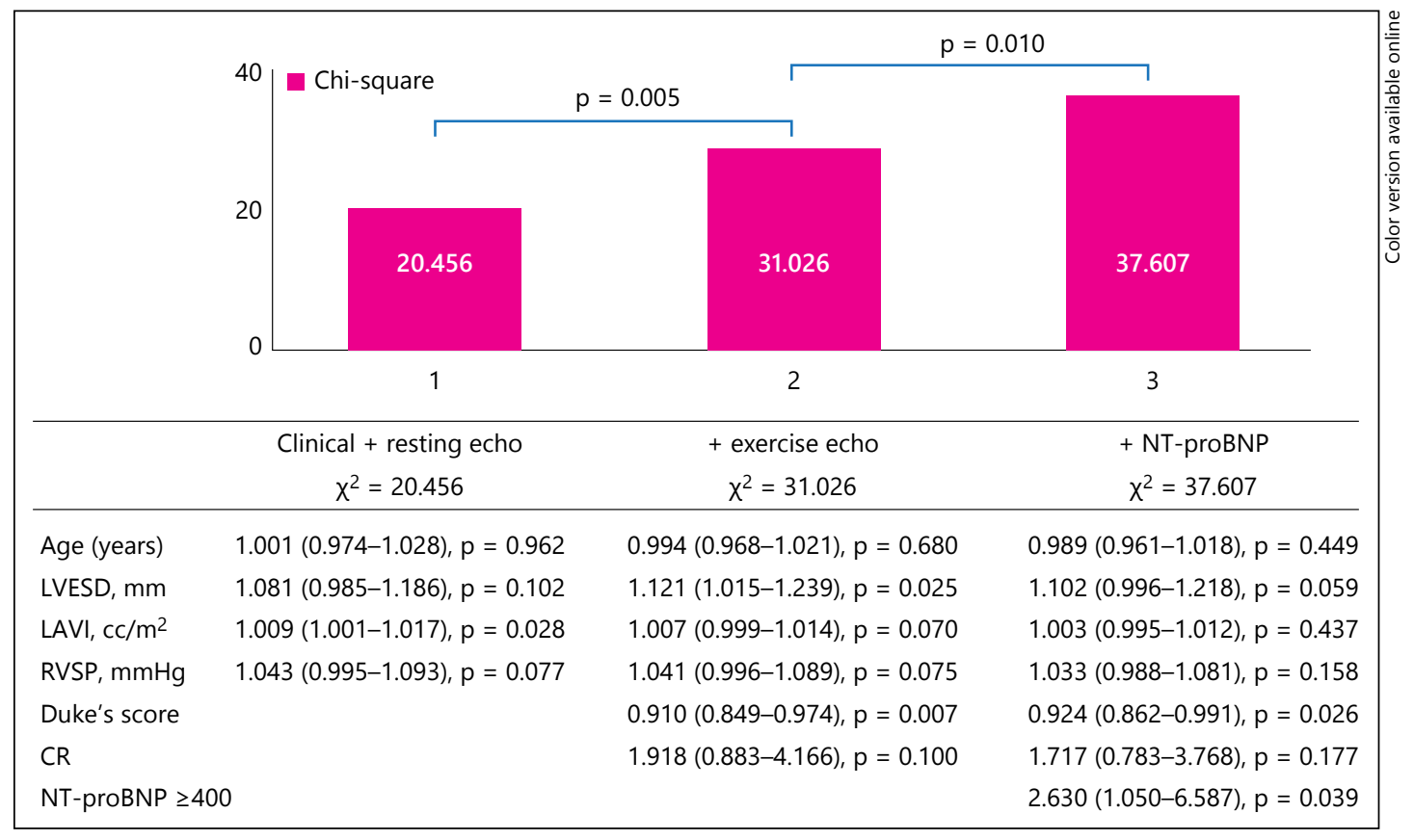

Fig. 1. The incremental value or exercise stress echocardiography parameters and NT-proBNP in the clinical determination (for Abstract of Sung-Ji Park).

\section{Sutureless Perceval Aortic Valve Prosthesis: An Evaluation of Postoperative Platelet Count}

Karl Christian König, Thorsten Wahlers, Jens Wippermann

University Hospital of Cologne, Köln (Cologne), Germany

Objective: The Sorin Perceval S (SP) sutureless bioprosthesis was developed as advancement to conventional biological stented aortic valve prostheses, especially for high risk Patients due to its fast and easy implantation technique. As successor model of the Sorin Freedom Solo (FS) stentless valve, we investigated whether there is a significant drop in postoperative platelet count, like it was observed with the FS valve. Methods: Between September 2012 and October 2013, 20 Patients were enrolled in our single centre SP study group and their data were analyzed in a prospective manner. For comparison 20 patients who received an conventional biological valve replacement with an Edwards Perimount (EP) aortic valve prosthesis during the same period were matched with the previous group in a retrospective manner. Results: Postoperative platelet count was observed for five days. At the nadir of the postoperative platelet count the measured mean total values are $114.1 \times 10^{3} / \mu \mathrm{l}$ in the SP group with a minimum of $42 \times 10^{3} / \mu \mathrm{l}$ and a maximum of $230 \times 10^{3} / \mu \mathrm{l}$ (POD 2) and $104.9 \times 10^{3} / \mu \mathrm{l}$ in the EP group with a minimum of $68 \times 10^{3} / \mu \mathrm{l}$ and a maximum of $119 \times 10^{3} / \mu \mathrm{l}$ (POD 1) $(p=0.556)$. In both groups seven patients had a postoperative platelet count below $100 \times 10^{3} / \mu \mathrm{l}$ with one patient of the SP group who had a platelet count below $50 \times 10^{3} / \mu$. None of the patients in both groups had a platelet count below the critical margin of $30 \times 10^{3} / \mu \mathrm{l}$. The relative platelet count at the nadir in the SP group was $43 \%$ of the initial preoperative value and $51 \%$ in the EP group.
Platelet counts recovered adequately in $80 \%$ of the patients of the SP group and in $66 \%$ of the patients of the EP group. Conclusion: There was no significant drop in platelet count observed in patients receiving SP aortic valve replacement in comparison to patients receiving conventional aortic valve replacement like it was seen with its predecessor the FS valve.

\section{Minimal Access Rapid Valve Deployment AVR Using the EDWARDS INTUITY Valve System}

Markus Schloemicher Peter Haldenwang, Baerbel Buchwald, Matthias Bechtel, Vadim Moustafine, Justus Thomas Strauch

Ruhr University Hospital Bergmannsheil, Bochum, Germany

Objectives: The EDWARDS INTUITY valve system combines the proven long-term safety and efficacy of the Carpentier-Edwards PERIMOUNT platform with recent innovations from transcatheter valve programmes. Since March 201254 implants were performed in our institution. This paper presents the first short term and 6 months clinical and hemodynamic results. Patients and Methods: The mean age was $75.5( \pm 6.2)$ years. A Euroscore II of $3.5 \% \pm 1.3 \%$ was found and $39 \%(n=21)$ were female. A clinical follow up for echocardiographic assessment of the valve performance was performed after 6 months. Preoperatively, 41 patients $(76 \%)$ were in NYHA class III or IV. Single AVR was performed using a partial upper sternotomy in all cases. A peak gradient of $69.5( \pm 23.7) \mathrm{mm} \mathrm{Hg}$ and a mean gradient of $46.7( \pm 17.9)$ 
were measured preoperatively. A mean effective orifice area of 0.8 $\mathrm{cm}^{2}( \pm 0.3)$ and an ejection fraction of $58.5 \%( \pm 15.0)$ could be found. Results: 30 -day mortality rate was $1.9 \%(n=1)$. One patient died because of severe postoperative bleeding from aortotomy. In one procedure intraoperative severe paravalvular leakage led to implantation of a conventional bovine pericardial valve. The peak and mean postoperative transprosthetic gradients were determined to be $21.9( \pm 7.6) \mathrm{mm} \mathrm{Hg}$ and $12.1( \pm 4.2) \mathrm{mm} \mathrm{Hg}$, respectively. A mean efective orifice area of $1.8 \mathrm{~cm}^{2}( \pm 0.3)$ was determined. No higher grade regurgitation $(\mathrm{AI}>+1)$ occured. Late mortality rate was $5.7 \%(3 / 53)$. The specific causes of late deaths were reported to be urogenital infection with consecutive septicemia $(n=1)$ and heart failure $(n=2)$ After 6 months the mean transprosthetic gradients were $19.0( \pm 6.3) \mathrm{mm} \mathrm{Hg}$ and $10.0( \pm 2.7) \mathrm{mm} \mathrm{Hg}$, respectively. The mean EOA of $1.8( \pm 0.3)$ remained unchanged. The mean implantation time amounted $9( \pm 3)$ minutes. The mean cross clamp time was $29 \pm 17$ minutes. An average bypass time of $62( \pm 16)$ minutes was seen. Conclusion: Short term results show excellent hemodynamic results. A satisfying reduction of cross clamp and bypass times could be achieved. Nevertheless future follow up investigation has to be awaited to gain more data concerning durability and safety issues.

\section{Novel TRI ${ }^{\mathrm{TM}}$ Heart Valve Biomaterial Resists Calcification and Elicits Reduced Inflammatory Response}

Hobey Tam, Narendra Vyavahare

Clemson University, Clemson, SC, USA

Objective: We have developed a novel, proprietary fabrication method (TRI ${ }^{\mathrm{TM}}$ ) that utilizes carbodiimide crosslinking chemistry, neomycin trisulfate, and pentagalloyl glucose to stabilize extracellular matrix components in soft tissues to resist (1) calcification and (2) structural degradation. In this study, we demonstrate that porcine aortic valve leaflets treated with $\mathrm{TRI}^{\mathrm{TM}}$ resist calcification in vivo and may be more biocompatible than glutaraldehyde (GLUT) leaflets, the current market gold standard. Methods: Freshly harvested porcine aortic heart valve leaflets were treated with either $0.6 \%$ GLUT or TRI ${ }^{\mathrm{TM}}$. Differential scanning calorimetry (DSC) and enzymatic degradation was used to analyze samples for tissue stability. Treated leaflets were subcutaneously implanted into juvenile rats for 90 days to assess in vivo biocompatibility. Pre and post implanted samples were analyzed for mineralization via inductively coupled plasma mass spectrometry (ICP) and Alizarin red staining. Hematoxylin and Eosin staining ( $\mathrm{H} \& \mathrm{E})$ was utilized to evaluate fibrous capsule thickness, macrophage activity, and foreign body giant cell formation. Lectin histochemistry was used to detect Gala epitope left in the tissue. Immunohistochemistry (IHC) was used to detect macrophages and T-cells in explants. Reuslts: H\&E on GLUT explants revealed chronic persistence of macrophages, formation of foreign body giant cells, and moderate fibrous capsule thickness. Dark, black nodules were found throughout the GLUT explants that are not clear as to if they are calcification nodules or if it is phagocytic/immunogenic activity. IHC is being performed to determine if these are host macrophages/T-cells
Table 1. Tissue stability assessment (for Abstract of Hobey Tam)

\begin{tabular}{lllll}
\hline & $\begin{array}{l}\text { Collagenase } \\
\text { Stability } \\
\text { (\% wt. loss) }\end{array}$ & $\begin{array}{l}\text { Elastase } \\
\text { Stability } \\
\text { (\% wt. loss) }\end{array}$ & $\begin{array}{l}\text { GAGase } \\
\text { Stability } \\
(\% \text { GAGs lost) }\end{array}$ & $\begin{array}{l}\mathrm{T}_{\mathrm{d}} \text { by } \\
\left({ }^{\circ} \mathrm{C}\right)\end{array}$ \\
\hline GLUT & $1.52 \pm 1.07$ & $16.8 \pm 2.1$ & $57.3 \pm 4.9$ & $89.7 \pm 0.9$ \\
TRI & $0.42 \pm 0.42$ & $4.6 \pm 1.9^{*}$ & $9.5 \pm 0.4^{*}$ & $90.1 \pm 1.2$ \\
\hline
\end{tabular}

* Indicates significant difference $(\mathrm{p}<0.05)$ from GLUT.

infiltrating the implant. TRI ${ }^{\mathrm{TM}}$ elicited little inflammatory response with minimal fibrous capsule formation and no morphological changes within the implant. No Gala was detected in either GLUT or TRI ${ }^{\mathrm{TM}}$ leaflets. Conclusions: Our data suggest that TRI ${ }^{\mathrm{TM}}$ is a more stable biomaterial, effectively resists calcification, and elicits a markedly reduced inflammation response from the body thus TRI ${ }^{\mathrm{TM}}$ may be a more suitable biomaterial than GLUT for bioprosthetic heart valve fabrication.

\section{A Prospective Study to Evaluate Minimally Invasive Right Thoracotomy Versus Conventional Sternotomy Approach for Mitral Valve Repair in Rheumatic Patients}

\section{Shamsher S. Lohchab}

PGIMS, Rohtak, India

Objective: This study was planned to evaluate the feasibility of mitral valve repair through minimally invasive right anterolateral thoracotomy approach and compare the outcomes with conventional sternotomy approach. Method: From May 2012 to October 2013.25 patients underwent mitral valve repair through limited right anterolateral thoracotomy (Group 1). Various clinical outcome parameters were compared with a 25-patient (Group 2) sternotomy. The age and sex distribution in two groups were comparable. All patients in both groups were having similar spectrum of RHD and functional status. Different repair techniques were used with rigid ring annuloplasty. TEE was used. Results: There was no operative mortality. Clinical parameters were better with group 1 compared to group 2, like ICU stay (68.84 hrs vs. $88.82 \mathrm{hrs),} \mathrm{hos-}$ pital stay (5.4 days vs. 9.5 days), chest tube drainage $(375.60 \mathrm{ml}$ vs. $536.5 \mathrm{ml}$ ), chest tube removal ( $55.96 \mathrm{hrs}$ vs. $65.78 \mathrm{hrs}$ ), incision length $(7.4 \mathrm{~cm}$ vs. $14.2 \mathrm{~cm})$. Cross clamp time and bypass time were higher in group 1. Superficial groin wound discharge was in one in group 1. Outcome in term of mitral valve function was comparable (significant MS, MR 4\%, $0 \%$ group 1 vs. $4 \%, 4 \%$ group 2 at maximum follow up of 18 months. Conclusion: Mitral valve repair is feasible and safe with minimally invasive thoracotomy approach with equivalent functional results and is better with respect to cosmesis, incision length, ICU stay, hospital stay, early mobility and patient satisfaction compared to sternotomy. 


\section{Simple and Reproducible Loop Technique for Mitral Valve Repair Using Preoperative Echocardiographic Determination}

\section{Hiroyuki Nishi, Koichi Toda, Shigeru Miyagawa, \\ Yasushi Yoshikawa, Satsuki Fukushima, Masashi Kawamura, Daisuke Yoshioka, Tetsuya Saito, Takayoshi Ueno, Toru Kuratani, Yoshiki Sawa \\ Osaka University Graduate School of Medicine, Osaka, Japan}

Objective: Although the Gore-Tex loop technique has been widely adapted for mitral valve repair, it remains difficult to determine the appropriate length, position, and number of loops prior to surgery. We assessed our simple and reproducible method to determine appropriate loop length and number of loops required using preoperative 3D echocardiography. Methods: Twenty patients (males, $13 ; 52.8 \pm 16.6$ years) underwent mitral valve repair using our strategy. We assessed the prolapse position using $3 \mathrm{D}$ echocardiography to select the papillary muscle for loop attachment, and the number and positions of the loops (figure 1a). Then, we measured the distance between the papillary muscle tip and level of the mitral annulus (annulus height length: $\mathrm{AH}$, figure $1 \mathrm{~b}$ ). the loop positions were adjusted intraoperatively using a 'ONEKNOT technique'. We assessed mitral valve repair results, and preoperative $\mathrm{AH}$, actual loop length (LL), and postoperative coaptation length (CL). Results: (1) Seven patients had anterior leaflet prolapse, while 5 had posterior and 8 bileaflet prolapse. The base of the loops was attached to the anterior papillary muscle in 16 and posterior in 7 patients, with the other end attached to the anterior leaflet in 7, posterior in 5, and both in 8. (2) Mean AH length and actual LL was $22.3 \pm 4.7$ and $19.6 \pm 5.9 \mathrm{~mm}$, respectively. LL in most cases was within -3 to $+4 \mathrm{~mm}$, as compared to AH. Postoperative CL was $10.2 \pm 3.7 \mathrm{~mm}$. CL in patients with LL shorter than $\mathrm{AH}$ tended to be deeper as compared to those with LL longer than $\mathrm{AH}$. (3) All mitral valve repairs were successful. Postoperative echocardiography MR grade was none in 12 and trivial in 7 . LL in 1 patient with mild postoperative MR was $7 \mathrm{~mm}$ longer as compared to $\mathrm{AH}$. Conclusions: Using our comprehensive strategy, it was possible to perform the present simple and reproducible mitral valve method using loops. The appropriate length seemed to be 3-5 mm shorter than the distance from the papillary muscle tip to level of the mitral annulus.

\section{Brachiocephalic Artery Access in TAVI: A Safe and Feasible Alternative for Retrograde Access}

\section{Tine E. Philipsen, Valérie M. Collas, Johan M. Bosmans,} Marc J. Claeys, Inez E. Rodrigus

UZA - Antwerp University Hospital, Edegem, Belgium

Objective: Transcatheter aortic valve implantation (TAVI) is an accepted treatment for selected high risk patients with severe aortic stenosis. Vascular access and access related complications remain a key point of concern. We report our initial experience with the brachiocephalic artery access, a new approach for retrograde TAVI. Methods: The ideal patient-tailored access is decided based on the pre-procedural thoracic angio-CT scan. In patients with poor iliofemoral access (small vessels, severe atherosclerosis, previous vascular surgery or stenting) or with patent LIMA graft after previous CABG, the open brachiocephalic artery access is preferred. The approach can be either suprasternal or through upper ministernotomy. Results: In 179 subsequent TAVI procedures (Medtronic CoreValve ${ }^{\circledR}$ ) performed by retrograde access in our center, the iliofemoral access was not deemed feasible in 31 patients $(17.3 \%)$. As an alternative, we preferred the brachiocephalic

Table 1. Baseline characteristics (for Abstract of Tine E. Philipsen)

\begin{tabular}{lc}
\hline Male (\%, N) & $80 \%(12)$ \\
Age (mean, y) & 79.1 \\
BSA (mean, ${ }^{2}$ ) & 1.81 \\
log EuroScore (mean) & 27.32 \\
STS score (mean) & 7.519 \\
NYHA class 3-4 (\%, N) & $93.3 \%(14)$ \\
AVA (mean, $\mathrm{cm}^{2}$ ) & 0.61 (range 0.4-0.86) \\
Peak gradient aortic valve (mean, mm Hg) & 69.1 (range 39-106) \\
Mean gradient aortic valve (mean, mm Hg) & $45($ range 30-69) \\
Peripheral arterial disease (\%, N) & $93.3 \%(14)$ \\
Porcelain aorta (\%, N) & $33.3 \%(5)$ \\
Previous vascular surgery (\%, N) & $33.3 \%(5)$ \\
Previous PCI (\%, N) & $33.3 \%(5)$ \\
Previous CABG (\%, N) & $60.0 \%(9)$ \\
Patent LIMA graft $(\%, \mathrm{~N})$ & $53.3 \%(8)$ \\
Patent venous grafts aorta (\%, N) & $53.3 \%(8)$ \\
\hline
\end{tabular}

Fig. 1. (for Abstract of Hiroyuki Nishi).
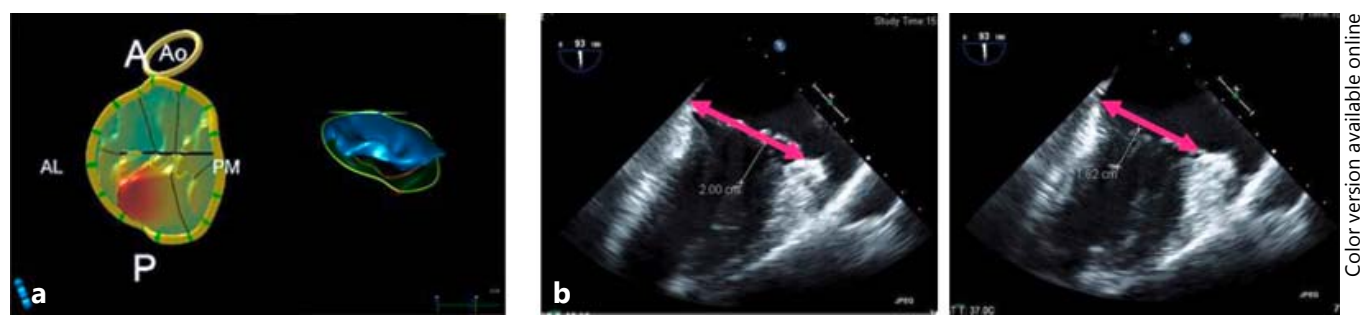
artery in 15 patients. In $73 \%$, the suprasternal approach provided excellent access. Patient baseline characteristics are given in table 1 . The procedural success rate was $100 \%$, we saw no intraprocedural mortality, no conversion to open surgery was needed and all valves were well positioned. Mean length of hospital stay was 9 days (range 5-14). Early safety was excellent, no periprocedural myocardial infarction, stroke, transient ischemic attack, acute kidney injury, major vascular or major bleeding complications were seen. 2 patients needed a permanent pacemaker within 2 days post TAVI because of a new third-degree AV block. Echocardiography at discharge showed one mild to moderate paravalvular aortic valve insufficiency and overall low mean gradients over the valve (range 6-14 mm Hg (mean 9)). Conclusions: TAVI implantation trough the brachiocephalic artery is safe and feasible. The surgical access is easily performed. The distance between the point of access and the aortic valve annulus is short, hereby improving catheter stability and accurate implantation. It therefore is a good option in patients without femoral access and might even be a good alternative for the transapical approach.

\section{Redo-Aortic Valve Replacement Versus TAVR in Patients with Patent Grafts}

\section{Renzo Pessotto, Neal Uren, Andrew Flapan, Sai Prasad, David Northridge, Kirsty Stewart}

Edinburgh Royal Infirmary, Edinburgh, United Kingdom

Objective: Transcatheter aortic valve replacement (TAVR) has been shown to be successful in high risk patients while redo-aortic valve surgery (redo-AVR) in patients with previous coronary artery bypass grafting $(\mathrm{CABG})$ and patent grafts carries a high mortality. To define the best treatment strategy for this cohort of patients, we reviewed our experince with TAVR and redo-AVR at the Edinburgh heart Centre. Methods: From January 2011 to December 2013, 86 consecutive patients underwent either TAVR (43) or redo-AVR (43) at our Insitution. All inoperable patients and those considered too high risk for redo-AVR underwent TAVR. Outcomes were prospectively collected. Results: TAVR patients were significantly older (mean age 80 yrs vs. 73 ) and had a higher logistic euroscore (30.9 vs. 9.4). The 30-day mortality $(4.6 \% v s .7 \%)$ and the incidence of debilitating strokes (2.3 vs. $4.6 \%$ ) were significantly lower in TAVR. Following the procedure, TAVR pts had significantly shorter ITU (2.3 vs. 6.2 days) and hospital stays ( 4.5 vs. 18 days). Conclusions: TAVR should be considered in all patients with severe AS and patent grafts. Redo-AVR should be considered in younger pts in whom durability of the TAVR might be a concern or whenTAVR is not feasible.

\section{Concomitant Bypass Grafts do not Alter Early or Long-term Outcomes of Patients Undergoing Isolated Aortic Valve Replacement}

\author{
Mark A. Groh ${ }^{1}$, Steve W. Ely' ${ }^{1}$, Oliver A. Binns ${ }^{1}$, Alan M. Johnson ${ }^{1}$, \\ Wendy Westling ${ }^{2}$, Gerard L. Champsaur ${ }^{2}$
}

${ }^{1}$ Asheville Heart, Asheville, NC, USA; ${ }^{2}$ Mission Hospital-Clinical Research, Asheville, NC, USA

Background: Perceived increased risk in patients requiring coronary artery bypass grafting (CABG) during surgical aortic valve replacement (SAVR) has led some to advocate a hybrid approach with percutaneous coronary intervention (PCI) and SAVR as an advantage over CABG with SAVR. We reviewed our current long term outcomes in patients undergoing SAVR with and without CABG to assess the contemporary risk in these patients. Methods: A cohort of 956 consecutive patients who underwent SAVR \pm CABG between January, 2008 and June, 2013 were retrospectively reviewed. Intervention was a redo in 152 cases (16\%). Other variables are depicted in table 1 . Concomitant CABG was performed in 442 patients (46\%, Group I), and AVR alone in 514 patients (Group II). Results: Early preoperative morbidity was not statistically different between Groups I and II: atrial fibrillation, prolonged ventilation, renal failure, reoperation for bleeding, and TIA. Only stroke rate was higher in Group I receiving CABG $(1.75 \%$ vs. $0.19 \%, \mathrm{p}<0.01)$. Mortality was not significantly different between the 2 groups: $2.94 \%$ in Group I and $1.56 \%$ in Group II $(\mathrm{p}=0.13)$ despite a predicted higher mortality in Group I. Using multivariate logistic regression analysis, redo surgery, presence of cardiogenic shock and higher trans-aortic gradient were independent predictors of early mortality while age, chronic lung disease, and diabetes were predictors for late mortality. During a mean follow-up of 33.1 months, cumulative probability of survival at 5 years (Kaplan-Meier, fig. 1 ) was $76.9 \%$ and $81.6 \%$ for patients in Group I and II, respectively (Log-Rank test: $\mathrm{p}=0.47$ ). Conclu-

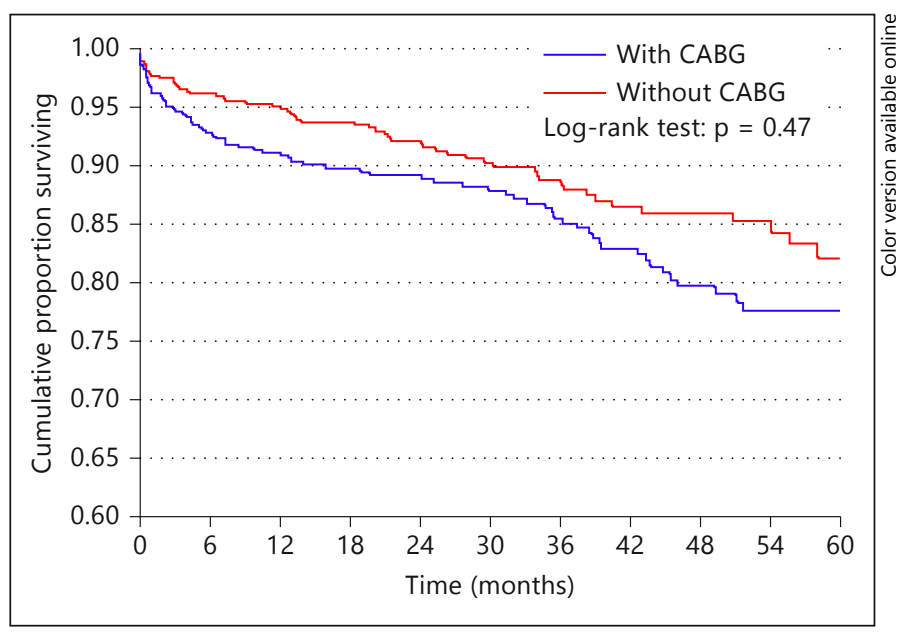

Fig. 1. Comparative Cumulative Proportion Surviving (KaplanMeier) after Isolated Aortic Valve Replacement with or without Concomitant CABG (for Abstract of Mark A. Groh). 
Table 1. Patient characteristics in 956 cases of SVR with or without CABG (for Abstract of Mark A. Groh)

\begin{tabular}{lllc}
\hline Variable & $\begin{array}{l}\text { Mean, Group I, } \\
\text { SVR+CABG }\end{array}$ & $\begin{array}{l}\text { Mean, Group II, p value } \\
\text { SVR alone }\end{array}$ & \\
\hline Age, years & 72.8 & 66.35 & $<0.01$ \\
EF, \% & 51.22 & 51.65 & 0.5 \\
LOS, days & 9.28 & 7.58 & $<0.01$ \\
MPG, mm Hg & 41.45 & 47.61 & $<0.01$ \\
Perfus. time, min & 140.34 & 98.89 & $<0.01$ \\
XClamp. time, min & 108.67 & 71.89 & $<0.01$ \\
Expected mortality & 0.0424 & 0.0263 & $<0.01$ \\
& CI 0.0378-0.047 & CI 0.0238-0.029 \\
\hline
\end{tabular}

sions: When considering revascularization in patients undergoing SAVR, CABG does increase bypass and cardiac exclusion times, as well as ICU and total length of stay. However, early and late mortality are not significantly altered by a single surgical procedure combining SAVR and CABG, with excellent long term durability.

\section{Alternative Access in Transcatheter Aortic Valve Implantation}

\author{
Tine E. Philipsen, Valérie M. Collas, Johan M. Bosmans, \\ Marc J. Claeys, Inez E. Rodrigus
}

UZA - Antwerp University Hospital, Edegem, Belgium

Introduction: The most common approach in transcatheter aortic valve implantation (TAVI) is no doubt the transfemoral. However, in case of atherosclerosis, small vessels or previous peripheral arterial surgery, transfemoral access is no option. Multiple alternative approaches have been described. In patients not suitable for transfemoral TAVI, we opt for an open approach through either the brachiocephalic artery or the ascending aorta. Methods: The brachiocephalic artery can mostly be accessed suprasternally. In case of calcification or small caliber of the brachiocephalic artery or presence of a hostile neck (eg previous cervical surgery), the ascending aorta may be the preferred access site. Results: In our series of 179 subsequent TAVI procedures (Medtronic CoreValve $\left.{ }^{\circledR}\right), 148$ patients were treated transfemorally. No major vascular complications were seen. In 36 patients (24.3\%) minor access related vascular injury necessitated further intervention (surgical repair (4.05\%), femoral stenting (11.49\%), femoral dilatation $(4.05 \%)$, thrombin injection $(4.73 \%))$. Thirty-one valves were implanted trough non-femoral access because of severe femoroiliac disease. In our early experience, 9 patients were treated by trans-subclavian access. In one case, periprocedural occlusion of a patent LIMA graft caused myocardial ischaemia, so this approach is no longer our primary choice. More recently, the open brachiocephalic access was successfully used in 15 patients. Eight had previous CABG with patent LIMA grafts, and 14 patients had severe femoroiliac atherosclerosis. In 11 of these patients, the brachiocephalic artery could be accessed suprasternally. Seven valves were implanted by direct aortic access after partial sternotomy in patients with both patent LIMA graft after previous CABG and severe peripheral atherosclerosis. No procedural or vascular complications were seen in the brachiocephalic or direct aortic access. Conclusion: TAVI is an accepted alternative to surgical AVR in high risk patients. Brachiocephalic and transaortic access are safe and feasible if transfemoral TAVI is contra-indicated, and might even be valid alternatives for transapical approach. The distance from the brachiocephalic artery or ascending aorta to the aortic valve annulus is small, hereby increasing delivery catheter stability and accurate valve positioning. Up to now, we did not decline any patient for TAVI treatment based exclusively on the absence of suitable access.

\section{AVIATOR Initiative: Aortic Valve Insufficiency and Ascending Aorta Aneurysm International Registry}

Frederiek De Heer ${ }^{1}$, Alain Berrebi ${ }^{2}$, Michael Borger ${ }^{3}$, Olivier Bouchot ${ }^{4}$, Duke Cameron ${ }^{5}$, Ruggero De Paulis ${ }^{6}$, Isabella Di Centa ${ }^{7}$, Laurent De Kerchove ${ }^{8}$, Gebrine El Khoury ${ }^{8}$, Ismail El-Hamamsy ${ }^{9}$, Jolanda Kluin ${ }^{1}$, Gianclaudio Mecozzi ${ }^{10}$, Wuliya Mijiti ${ }^{11}$, Jan Nijs ${ }^{12}$, Yutaka Okita ${ }^{13}$, Carlos Porras ${ }^{14}$, Hans-Joachim Schäfers ${ }^{15}$, Hartzell Schaff ${ }^{16}$, Malakh Shrestha ${ }^{17}$, Johanna Takkenberg ${ }^{18}$, Jean Louis Vanoverschelde ${ }^{8}$, Jan Vojacek ${ }^{19}$, Emmanuel Lansac ${ }^{2}$

${ }^{1}$ University Medical Center Utrecht, Utrecht, Netherlands; ${ }^{2}$ Institut Mutualiste Montsouris, Paris, France; ${ }^{3}$ Leipzig Heart Center, Leipzig, Germany; ${ }^{4}$ Centre Hospitalier Universitaire Dijon, Dijion, France; ${ }^{5}$ Johns Hopkins Hospital, Baltimore, MD, USA; ${ }^{6}$ European Hospital, Rome, Italy; ${ }^{7}$ Hopital Foch, Suresnes, France; ${ }^{8}$ Cliniques Universitaires Saint-Luc, Brussels, Belgium; ${ }^{9}$ Montréal Heart Institute, Montréal, QC, Canada; ${ }^{10}$ Medisch Spectrum Twente, Enschede, Netherlands; ${ }^{11}$ People Hospital, Urumchi-Xinjiang, China; ${ }^{12}$ University Hospital, Brussels, Belgium; ${ }^{13}$ Kobe University, Kobe, Japan; ${ }^{14} \mathrm{Hospital}$ universitario Virgen de la Victoria, Malaga, Spain; ${ }^{15}$ Saarland University Medical Center, Homburg/Saar, Germany; ${ }^{16}$ Mayo Clinic, Rochester, MN, USA; ${ }^{17}$ Hannover Medical School, Hannover, Germany; ${ }^{18}$ Erasmus University Medical Center, Rotterdam, Netherlands; ${ }^{19}$ University Hospital Hradec Kralove, Hradec Kralove, Czech Republic

Objective: Current guidelines for patients with aortic valve insufficiency and/or ascending aorta aneurysm are based on small single expert center case series with a limited follow-up duration. The Society of Heart Valve Disease aortic valve repair working group established the international prospective multicentre registry AVIATOR in 2013 to provide sufficient patient numbers, to address key epidemiological and therapeutic issues and to standardize indications for surgery as well as the place of repair versus replacement in aortic valve surgery. Methods: All patients, aged 18 years or older, operated for ascending aorta aneurysm (including root and/or supra coronary aorta and aortic dissection) and/or isolated aortic insufficiency (including congenital mixed aortic valve disease), should be enrolled in an intention to treat design. Surgical techniques include valve repair and replacement, valve sparing aortic root replacement and Bentall procedures. Baseline, 
procedural and early outcome data are collected prospectively during hospital stay. Thereafter annual follow-up will take place. Results: The Aviator registry is open to all centers willing to participate to this international collaborative effort with a dedicated online database for each center allowing state-of-the-art evaluation of valve related events. Currently 9 cardio-thoracic surgery centers from France; the Netherlands; Germany; Spain, Belgium, Czech Republic and Slovakia enrolled in the database. They included 140 patients thus far. A project update is given at this meeting. Conclusions: The international prospective multicentre AVIATOR registry will allow for acceleration of knowledge on the determinants of outcome in patients with aortic valve insufficiency and/or ascending aorta aneurysm. By combining surgical experience from multiple centers, and applying uniform definitions of echo and outcome parameters, it will become possible to provide a solid evidence base to clarify and standardize the place of repair versus replacement in aortic valve surgery.

\section{Effect of the Prosthesis-Patients Mismatch on Long Term Clinical Outcome in Patients with AVR For Aortic Stenosis with Small Prosthetic Valve ( $\leqq 19 \mathrm{~mm}$ )}

Hiroyuki Saisho, Koichi Arinaga, Satoshi Kikusaki, Kumiko Wada, Ryusuke Mori, Toru Takaseya, Koji Akasu, Tomokazu Kosuga, Satoru Tobinaga, Hidetoshi Akashi, Hiroyuki Tanaka

Kurume University, Kurume, Japan

Background: The clinical effects of PPM after AVR remain controversial. We evaluated the impact of PPM on clinical outcomes after AVR with small prosthetic valve $(\leqq 19 \mathrm{~mm})$ in patients with aortic stenosis (AS). Methods: We analyzed data from 130 patients with AS who underwent AVR with prosthesis $\leqq 19 \mathrm{~mm}$ between January 2003 and August 2013. 102 patients had bioprosthetic valve (CEP 32, CEP Magna 52, Trifecta 6, Mitroflow 10, Mosaic 2 ) and 28 patients had mechanical valve (19 mmSJM Regent 4, $19 \mathrm{~mm}$ ATS $6.18 \mathrm{mmATS}$ AP $5.17 \mathrm{mmSJM}$ Regent $12.16 \mathrm{mmATS}$ AP 1). We classified them into 2 groups according to the grade of patient prosthesis mismatch (PPM) assessed by echocardiography at 2 weeks post-surgery, (group P:PPM $(+), \mathrm{iEOA}<0.85$, group $\mathrm{N}$ : no PPM, iEOA $\geqq 0.85$ ). Both clinical and echocardiographic assessments for indexed left ventricular mass (iLVM) were performed preoperatively, at 2 weeks post-surgery and during follow up period (mean follow up time 2.92 years $(0.5-10.9$ year)). Results: The mean age and BSA were 75.1 years old and $1.36 \mathrm{~cm}^{2} / \mathrm{m}^{2}$ respectively. 61 patients (46\%) had PPM (group P). iEOA in group P and $\mathrm{N}$ was $0.74 \pm 0.09$, and $0.97+0.13 \mathrm{~cm}^{2} / \mathrm{m}^{2}$ respectively. There were no differences in hospital mortality and morbidity in both groups. The 5, 10-year free rate of cardiac-related death was $98.1 \%, 87.2 \%$ in group $\mathrm{P}$ and $91.8 \%, 82.6 \%$ in group $\mathrm{N}$ respectively $(\mathrm{p}=0.26)$. There was no significant difference about the 5, 10-year free rate of admission for heart failure (group P: $83.2 \%, 83.2 \%$, group $\mathrm{N}$ : $95.8 \%, 95.8 \%$ respectively) $(\mathrm{p}=0.18)$. In both groups, preoperative increased iLVM was almost recovered to normal value at 3 months post-surgery ( 149 to $108 \mathrm{~g} / \mathrm{m}^{2}$ in group $\mathrm{P}, 150$ to $107 \mathrm{~g} / \mathrm{m}^{2}$ in group $\mathrm{N})$. No difference in $\mathrm{EF}$ and $\mathrm{LV}$ dimension during long term pe- riod was observed in both groups. Conclusions: $46 \%$ of patients who underwent AVR with small prosthetic valve had PPM after surgery. However there was no significant difference about echocardiographic variables and long term clinical outcome between 2 groups. Therefore, PPM due to small prosthesis implantation was not associated with poor clinical results.

\section{Edwards Intuity Sutureless Valve System: The Bioprosthesis of the Future?}

\section{Vlad Gariboldi, Sr., Alexis Theron, Dominique Grisoli, Laetitia Nee, Pierre Morera, Séverine Leroux, Frédéric Collart}

Hôpital adultes de la timone, Marseille, France

Objective: The Edwards Intuity prosthesis is a sutureless rapid deployment pericardial bioprosthesis design for faster procedures, requiring only three sutures in conjunction with expanded frame for secure annular placement, reducing procedural steps and time. We analyze the results of the first 54 patients implanted in our institution. Methods: From July 2012 to December 2013, 54 patients received an Intuity bioprosthesis. Data were collected prospectively. All patients had pre- and postoperative transthoracic (at one month follow-up). Results: There were 40 male with a mean age of $78.1 \pm 8$ years ( 50 to 88 years). One patient had a previous aortic valve replacement with a Freestyle prosthesis. Mean Euroscore 2 was $4.1 \pm 3.2 \%$ ( $0.81 \%$ to $9.5 \%)$. Mean corporeal surface was $1.84 \mathrm{~m}^{2}$. NYHA class was II in 27 patients and III in 25 patients. Mean serum level of creatinin was $84 \pm 18 \mu \mathrm{mol} / \mathrm{l}$ ( 67 to $127 \mu \mathrm{mol} / \mathrm{l})$. Five patients had permanent atrial fibrillation. All except one natives aortic valves were tricuspid. Procedures were performed by full sternotomy. Mean cardiopulmonary bypass time was $64 \pm 23 \mathrm{~min}$ (45 to $109 \mathrm{~min}$ ). Mean aortic cross-clamping time was $46 \pm 20 \mathrm{~min}$ (32 to $87 \mathrm{~min}$ ). The diameters of the prosthesis implanted were 19 in 13 patients, 21 in 18 patients, 23 in 11 patients, 25 in 3 patients and 27 in 1 patient. The delivery of the device was successful in $100 \%$ patients. Associated procedures consisted in coronary artery bypass in 18 patients (33\%). One patient died. Mean duration of ICU stay was $3.4 \pm 2.1$ days. Mean duration on in-hospital stay was $11 \pm 2.9$ days. Significant improvements in transthoracic echocardiography parameters were observed between preoperative versus postoperative mean $\pm \mathrm{SD}$ measurements of: Vmax $(4.8 \pm 0.8 \mathrm{~m} / \mathrm{sec}$ versus $2.2 \pm 0.5 \mathrm{~m} / \mathrm{sec}$, respectively; $\mathrm{p}<0.001)$; aortic valve area $\left(0.7 \pm 0.2 \mathrm{~cm}^{2}\right.$ versus $1.8 \pm 0.5 \mathrm{~cm}^{2}$, respectively; $\left.\mathrm{p}<0.001\right)$; aortic valve area index $\left(0.4 \pm 0.1 \mathrm{~cm}^{2} / \mathrm{m}^{2}\right.$ versus $1.0 \pm 0.3 \mathrm{~cm}^{2} / \mathrm{m}^{2}$, respectively; $\mathrm{p}<0.001)$; mean transaortic gradient $(58 \pm 21 \mathrm{~mm} \mathrm{Hg}$ versus $12 \pm 4$ mm Hg, respectively; $\mathrm{p}<0.001)$. Conclusions: Edwards's Intuity bioprosthesis provides excellent early clinical and echocardiographic results. This device should enhance minimal invasive aortic valve replacement. Midterm results should be evaluated. 


\section{Pre-Clinical Study of Active Mitral Ring for the Percutaneous Treatment of Residual and Recurrent Mitral Regurgitation}

\section{Giuseppe Siniscalchi, Didier Locca, Fabrizio Gronchi, Enrico Ferrari, Rene Pretre, Piergiorgio Tozzi}

University Hospital Lausanne, Lausanne, Switzerland

Objective: The EVEREST study recently outlined the incidence of recurrent mitral regurgitation after surgical repair being higher than is traditionally thought thus affects a patient's clinical outcome. There are few alternatives to a second open heart surgery to treat residual and recurrent mitral regurgitation. Post-implant adjustable mitral rings potentially address this issue, allowing the reshaping of the annulus on the beating heart and avoiding a second surgical approach. This manuscript reports the results of the pre-clinical study of new mitral ring allowing valve geometry remodeling after implant. Methods: The device consists of two concentric rings: one internal and flexible, sutured to the mitral annulus and a second, external and rigid. A third element is inserted between the two rings modifying the shape of the flexible ring. This third element is remotely activated using a balloon similar to that used for coronary angioplasty. Animal model: in adult pig, under $\mathrm{CPB}$ we shortened primary cordae of P2 segment to reproduce type III regurgitation and implanted the active ring. We used intracardiac ultrasound to assess mitral regurgitation and the efficacy of the active ring to correct it. Results: Severe mitral regurgitation $(3+$ and $4+)$ was induced in 12 animals, $54 \pm 6 \mathrm{~kg}$. The activation of the device allowed the reduction of mitral regurgitation in all cases; in nine animals the regurgitation disappeared, in tree animals, it was trivial. Conclusions: A post-implant adjustable mitral ring corrects severe mitral regurgitation through reversible modification of the annulus geometry on the beating heart. It will address the frequent and morbid issue of recurrent mitral valve regurgitation. The future looks towards, one unique surgical procedure laying the foundations for future percutaneous corrections.

\section{Aortic Valve Replacement in Octogenarians: Impact of Less Invasivity on Postoperative Outcome}

\section{Tarek Alameddine \\ Sultan Qaboos University Hospital, Muscat, Oman}

Objective: Due to increasing life expectancy in our countries, the number of octogenarian patients undergoing an open heart procedure is increasing. In these patients with high comorbidity less invasive procedures could probably improve the postoperative outcome. The aim of this study was to determine the effects of minimal access aortic valve replacement (AVR) in octogenarians on postoperative morbidity and mortality. Methods: Partial upper sternotomy has become the standard approach to isolated aortic valve surgery in patients without any previous cardiac surgery at our institution. Allmost $20 \%$ of the 353 patients who underwent AVR through a partial upper sternotomy between 2007 and 2013 were octogenarians. These 70 patients (16 male, 54 female) had a mean age of 81.8 years ( $80-95$ years). We reviewed retrospectively data on these patients. Results: The patients had a mean logistic Euroscore of $9.1(0.9-29.2)$ and a mean left ventricular ejection fraction of $54.7 \%$ (20-70\%). Mean cross clamp time and mean bypass time were $63.3 \mathrm{~min}(33-149 \mathrm{~min})$ and $108.5 \mathrm{~min}(52-290$ $\mathrm{min})$, respectively. In 3 patients $(4.3 \%)$ a conversion into full median sternotomy was necessary due to bleeding $(n=2)$ and low cardiac output $(\mathrm{n}=1)$. Mean ICU and total hospital stay were 2.8 and 11.7 days, respectively. Postoperative bleeding complications were not observed. Deep sternum infection occured in 2 patients $(2.8 \%)$. None of the patients had to be reoperated for prosthetic valve dysfunction or endocarditis. The hospital mortality rate was $5.7 \%$. Conclusions: AVR through a partial upper sternotomy in octagenarians is a safe and effective technique with less invasivity resulting in satisfying postoperative morbidity and mortality.

\section{The New Generation of Stented Aortic Pericardial Tissue Valves in Patients with Small Aortic Roots: Single Center Experience in 572 Patients}

\section{Reinhard Moidl, David Santer, Harald Pisarik, Sandra Folkmann, Gabriel Weiss, Martin Grabenwoeger}

General Hospital Hietzing, Vienna, Austria

Objective: The aim of our study was to compare the hemodynamic and clinical performances between different stented aortic tissue valves in patients with small aortic roots. Methods: Between 2005 and 2012, 572 consecutive patients with small aortic roots (mean age $74.6 \pm 9.0$ years, additional procedures $47.2 \%$, logistic predicted mortality LPM $=10.8 \pm 9.3 \% ; 1.3-78.5)$, received different types of size $19(\mathrm{n}=171)$ and $21(\mathrm{n}=401)$ tissue valves in our institution: Sorin Mitroflow ${ }^{\circledR}$ (MF) 149, CE Magna Ease ${ }^{\circledR}$ (ME) 119, CE Perimount Magna ${ }^{\circledR}$ (PM) 98, SJM Trifecta ${ }^{\circledR}$ (TF) 90, Medtronic Mosaic $^{\circledR}$ (M) 47, SJM Epic ${ }^{\circledR}$ (E) 42, CE Perimount ${ }^{\circledR}$ (P) 27. Patient prosthesis mismatch (PPM) was calculated and defined as mismatch if EOAI $\leq 0.85 \mathrm{~cm}^{2} / \mathrm{m}^{2}(\mathrm{PPM})$ and severe mismatch if EOAI $\leq 0.65 \mathrm{~cm}^{2} / \mathrm{m}^{2}$ (sPPM). Statistical analysis was performed with IBM SPSS statistics (Version21.0; Pearson's Chi-Square test). Results: Moderate and severe PPM was present in porcine aortic valves (PPM/ sPPM: M : 55\%/43\%; E: 74\%/37\%) and in bovine pericardial tissue valves (MF: 49\%/44\%, CEP: $26 \% / 0 \%$, PM: 45\%/1\%, TF: $21 \% / 0 \%$, ME: $16 \% / 0 \%)$. In patients received size 19 valves without PPM, the perioperative mortality was statistically significant decreased in isolated procedures (0\%, LPM 9.97\%; $\mathrm{p}<0.01)$. In contrast the mortality in patients with severe PPM is high and not statistically significant different between isolated and combined procedures ( 7.4 vs. 10\%; ns). In our experience the new generation of bovine pericardial tissue valves (CE Perimount ${ }^{\circledR}$, CE Perimount Magna ${ }^{\circledR}$, CE Magna Ease ${ }^{\circledR}$ and SJM Trifecta ${ }^{\circledR}$ ) showed the lowest incidence of moderate and severe PPM in the small sizes. With the use of these pericardial tissue valves, we further improved perioperative mortality in patients with small aortic roots $(2.9 \%$ vs. $4.3 \%, \mathrm{p}=0.029$; LPM 8.5\%, $\mathrm{p}=0.031)$. Conclusions: In our experience with aortic valve replacement in patients with small aortic roots, the use of pericardial tissue valves has further improved the perioperative outcome and hemodynamic performances; in particular the latest generation of pericardial tissues 
valves showed the lowest incidence of PPM in the cohort. We recommend pericardial tissue valves as the aortic valve substitute of choice in patients with small aortic roots.

\section{Gender Differences and Early Outcome After Concomitant Aortic Valve and CABG Surgery: A Nationwide Study}

\author{
Mostafa M. Mokhles ${ }^{1}$, Sabrina Siregar ${ }^{2}$, Michel Versteegh ${ }^{2}$, \\ Luc Noyez ${ }^{3}$, Bart van Putte ${ }^{4}$, Alexander B.A. Vonk ${ }^{5}$, \\ Jolien W. Roos-Hesselink ${ }^{1}$, Ad J.J.C. Bogers ${ }^{1}$, \\ Johanna J.M. Takkenberg ${ }^{1}$ \\ ${ }^{1}$ Erasmus University Medical Center, Rotterdam, Netherlands; \\ ${ }^{2}$ Leiden University Medical Center, Leiden, Netherlands; \\ ${ }^{3}$ Radboud University Medical Center, Nijmegen, Netherlands; \\ ${ }^{4}$ St Antonius Hospital, Nieuwegein, Netherlands; ${ }^{5}$ Free \\ University Medical Center, Amsterdam, Netherlands
}

Objective: Although gender-related differences in patient presentation, morbidity and mortality are well described for coronary artery bypass grafting (CABG), there is uncertainty about whether this also applies to patients undergoing concomitant aortic valve (AV) and CABG surgery. The aim of this study was to evaluate gender-related differences in patients undergoing concomitant AV and CABG surgery. Methods: All patients $(\mathrm{N}=5,867,67 \%$ males $(\mathrm{N}=$ $3,937)$ ) that underwent AV surgery (replacement: $\mathrm{N}=5,817,99.1 \%$; reconstruction: $\mathrm{N}=50,0.9 \%)$ with concomitant $\mathrm{CABG}$ in the Netherlands between January 2007 and December 2011 were included in this study. Differences in patient and procedural characteristics, and in-hospital outcome were compared between male en female patients. Results: Female patients were older (mean age, 75 vs. 72 years, $\mathrm{p}<0.001$ ) and had higher logistic EuroSCORE (median score, 8.1 vs. $5.5, \mathrm{p}<0.001)$. Male patients presented more often with chronic lung disease $(16.8 \%$ vs. $12.1 \%$; $\mathrm{p}<0.001)$, extracardiac arteriopathy $(18.9 \%$ vs. $12.9 \%$; $<<0.001)$, neurological dysfunction ( $3.5 \%$ vs. $2.3 \%$; $\mathrm{p}<0.001)$, prior cardiac surgery ( $4.7 \%$ vs. $3.3 \%$; $\mathrm{p}<$
$0.001)$, renal disease ( $2.7 \%$ vs. $1.4 \% ; \mathrm{p}<0.001)$, active endocarditis ( $1.0 \%$ vs. $0.4 \%$; $\mathrm{p}<0.001)$, moderate $(21.2 \%$ vs. $15.3 \%$; $\mathrm{p}<0.001)$ and poor $(5.7 \%$ vs. $2.5 \%$; p < 0.001$)$ left ventricular function. Female patients received more often stented $(81.3 \%$ vs. $73.6 \%$; $<<0.001)$ and stentless $(5.2 \%$ vs. $4.0 \%$; $\mathrm{p}<0.001)$ bioprostheses. Male patients, on the other hand, received more often a mechanical prosthesis $(21.7 \%$ vs. $12.3 \%$; $\mathrm{p}<0.001)$. Female patients underwent more often total venous grafting ( $30.0 \%$ vs. $26.3 \%$; $\mathrm{p}=0.003)$. In-hospital mortality was $4.1 \%(\mathrm{n}=239)$ and higher in female compared to male patients (OR 2.00, 95\% CI 1.44-2.79; $\mathrm{p}<0.001$ ). In males, the AUC for the logistic EuroSCORE was 0.78 (95\% CI 0.73-0.82) versus 0.69 (95\% CI 0.63-0.74) in females. The calibration of the logistic EuroSCORE model resulted in $p$-values of $p<0.001$ and $p=0.09$ for males and females, respectively. Conclusions: There are considerable gender differences in patient presentation, operative characteristics, and inhospital mortality in contemporary concomitant AV and CABG surgery. The predictive value of logistic EuroSCORE is substantially better in men. This calls for the development of gender specific risk stratification models for combined AV and CABG surgery.

\section{Valvular Interstitial Cells Suppress Calcification of Valvular Endothelial Cells}

\author{
Jesper Hjortnaes ${ }^{1}$, Kayle Shapero ${ }^{2}$, Josh Keegan ${ }^{3}$, \\ Claudia Goettsch", Jolanda Kluin' ${ }^{4}$, John E. Mayer', \\ Joyce Bischoff 5 , Elena Aikawa \\ 'University Medical Center Utrecht, Utrecht, Netherlands; \\ ${ }^{2}$ Children's Hospital Boston/Harvard Medical School, Boston, \\ MA, USA; ${ }^{3}$ Brigham and Women's Hospital, Boston, MA, USA; \\ ${ }^{4}$ Brigham and Women's Hospital/Harvard Medical School, \\ Boston, MA, USA; ${ }^{5}$ Children's Hospital Boston, Boston, MA, USA
}

Objective: Calcific aortic valve disease (CAVD) is the most common heart valve disease in the Western world. We previously proposed that valvular endothelial cells (VECs) replenish valve

Table 1. Multivariate analyses of risk factors associated with in-hospital mortality in male and female patients (for Abstract of Mostafa M. Mokhles)

\begin{tabular}{|c|c|c|c|c|}
\hline \multirow[t]{2}{*}{ Characteristics } & \multicolumn{2}{|l|}{ Males } & \multicolumn{2}{|l|}{ Females } \\
\hline & OR $(95 \% \mathrm{CI})$ & p-value & OR $(95 \% \mathrm{CI})$ & p-value \\
\hline Age & $1.07(1.03-1.10)$ & $<0.001$ & $1.05(1.01-1.09)$ & 0.007 \\
\hline Chronic lung disease & $2.23(1.42-3.50)$ & $<0.001$ & & \\
\hline Extracardiac arteriopathy & $2.00(1.29-3.09)$ & 0.002 & & \\
\hline Prior cardiac surgery & $3.80(2.08-6.95)$ & $<0.001$ & $5.90(2.88-12.12)$ & $<0.001$ \\
\hline Unstable angina & $3.29(1.38-7.82)$ & 0.007 & & \\
\hline \multicolumn{5}{|l|}{ LV function } \\
\hline Good & reference & & & \\
\hline Poor & $2.61(1.37-4.99)$ & 0.004 & & \\
\hline Pulmonary hypertension & $2.23(1.07-4.67)$ & 0.033 & & \\
\hline Emergent surgery & $3.14(1.29-7.62)$ & 0.012 & $5.75(2.49-13.29)$ & $<0.001$ \\
\hline Total venous graft & $1.19(1.00-1.41)$ & 0.045 & & \\
\hline Increasing number of venous anastomosis & & & $1.26(1.05-1.52)$ & 0.013 \\
\hline
\end{tabular}




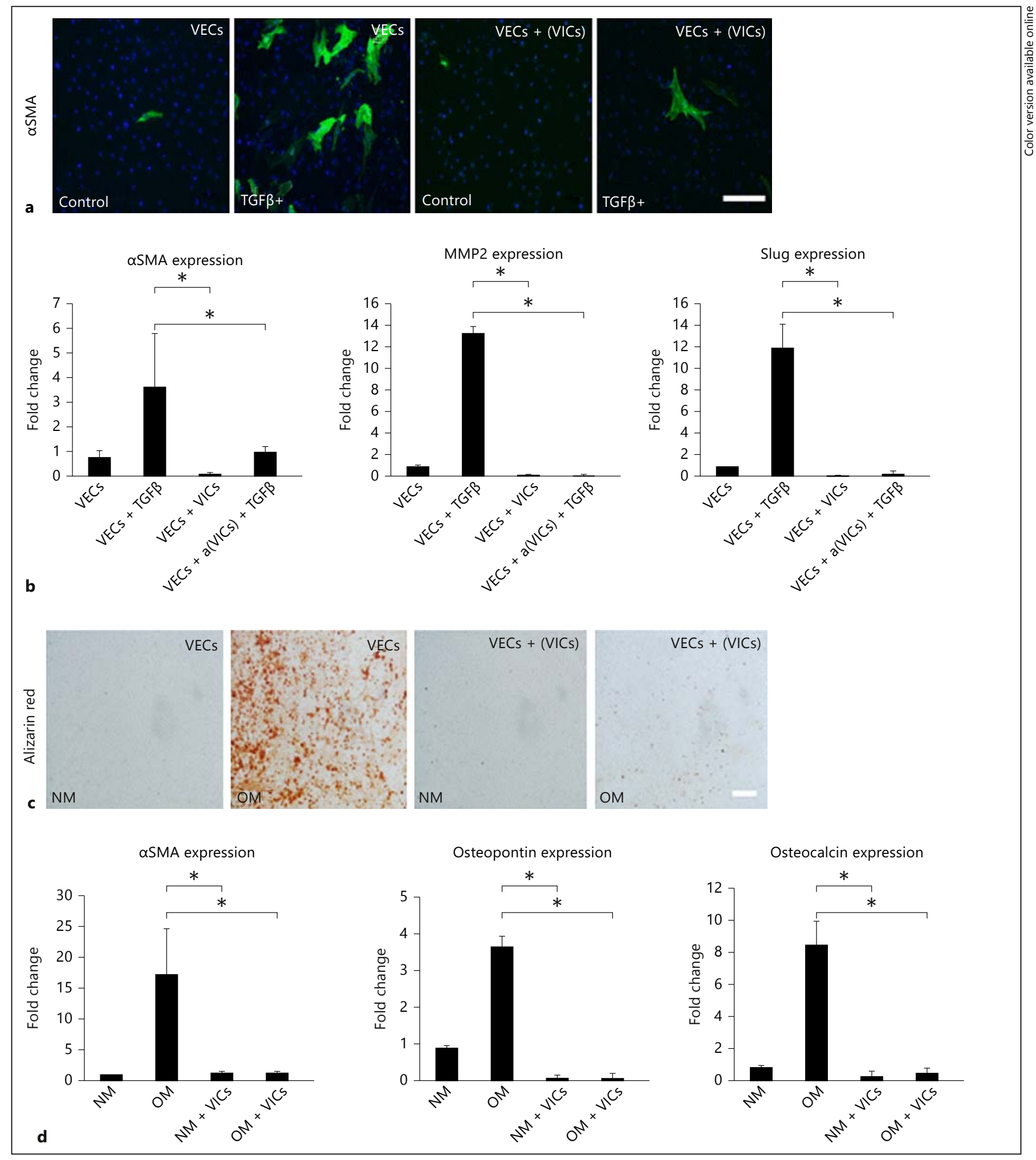

Fig. 1. (for Abstract of Lesper Hjortnaes). 
leaflets in response to injury via endothelial to mesenchymal transformation (EndoMT). In addition, it has been demonstrated that VECs could differentiate into osteoblastic cells. However, how this VEC plasticity interacts with valvular interstitial cells (VICs) with regards to valvular calcification is unknown. We hypothesized that aortic VECs can undergo osteogenic differentiation, but may be inhibited by VICs. Methods and Results: We used VEC clones that undergo TGF $\beta$-mediated EndoMT, confirmed by significantly increased expression, compared to control, of EndoMT markers aSMA (5.3 \pm 1.2$),$ MMP2 $(13.5 \pm 0.6)$ and Slug $(12 \pm 2.1)(\mathrm{p}<0.05)$ (fig. 1a, b). We further investigated EndMT in the presence of VIC derived from the same valve leaflets as the VECs. Clonal populations of VICs were isolated and characterized, and shown to express an activated, myofibroblast-like phenotype. The VICswere placed in co-culture with VECs using a transwell system, in normal media and media supplemented with TGF $\beta$. In the presence of VICs, TGF $\beta$-mediated EndoMT in VECs was inhibited, as shown by decreased expression of aSMA $(0.1 \pm 0.5)$, MMP2 $(0.1 \pm 0.1)$ and Slug $(0.2 \pm 0.2)(p<0.05)$. In addition, co-culture with VICs suppressed the migratory ability of TGF $\beta$-stimulated VECs. When cultured alone in osteogenic media, VECs demonstrated osteogenic changes (Alizarin Red), confirmed by significant increases in mRNA expression of osteocalcin $(8.6 \pm 1.3)$ and osteopontin $(3.7 \pm 0.3)$, whereas the presence of VICs inhibited VEC osteogenesis shown by decreased expression of osteocalcin $(0.4 \pm 0.1)$ and osteopontin $(0.2 \pm 0.1)(\mathrm{p}<0.05)$ (fig. 1c, d]. Time course analysis revealed that EndoMT precedes osteogenesis, demonstrated by an initial increase of aSMA and MMP2 at day 7 followed by an increase of osteopontin and osteocalcin at day 14. Conclusions: This study shows that activated VICs inhibit EndMT and osteoblastic differentiation of VECs. EndoMT may precede the onset osteogenesis in VECs. These results indicate importance of VEC-VIC interactions in valve homeostasis and suggest a role for VECs in early CAVD.

\section{RVOT Replacement with the Novel Vascutek RVOT ELAN Biplex ${ }^{\mathrm{TM}}$ Conduit: Initial Results in the First 12 Patients}

\section{loannis Tzanavaros}

Sana Herzchirurgie Stuttgart, Stuttgart, Germany

Objective: Vascutek RVOT ELAN conduit is used since October 2012 as a surgical procedure for the treatment of RVOTdysfunction. The RVOT elan ${ }^{\mathrm{TM}}$ is a bioprosthetic heart valve conduit constructed from a combination of a Vascutek Biplex ${ }^{\mathrm{TM}}$ Valsalva ${ }^{\mathrm{TM}}$ vascular graft and a Vascutek elan ${ }^{\mathrm{TM}}$ porcine stentless heart valve. The initial results of the first 12 patients are presented in this study. Methods: Since October 2012 RVOT replacement, using the novel Vascutek RVOT ELAN conduit, was performed in 12 patients. Primary diagnosis was tetralogy of Fallot $(n=5)$, pulmonary atresia with VSD $(n=1)$, pulmonary atresia $(n=1)$, pulmonary stenosis (PS) $(n=1)$, Truncus arteriosus $(n=1)$, CAVSD/PI $(\mathrm{n}=1)$, TGA/VSD/PS $(\mathrm{n}=2)$. The leading hemodynamic problem consisted of a pulmonary stenosis (PS) $(n=5)$, pulmonary regurgitation $(P R)(n=5)$ and a combined PS/PR lesion $(n=2)$. Results:
RVOT replacement was performed without complications in all patients. There was no mortality or morbidity perioperative and in follow up. There were 3 female and 9 male patients; the mean age was 13.1 years (range 23 months -52 years), the mean length was $139.8 \mathrm{~cm}$ (range $84-174 \mathrm{~cm}$ ) and the weight $43.1 \mathrm{~kg}$ (range 11.2$98 \mathrm{~kg}$ ). Echocardiography data showed excellent RV-PA gradient in all patients. There was no residual pulmonary regurgitation in all 12 patients. In one patient stenting of the right pulmonary artery was performed due to a not detected stenosis before the conduit implantation. During the short-term follow-up of 4, 6 months in mean (range 1-14 months) there was no change in the immediate results. Conclusion: RVOT replacement, using the novel Vascutek RVOT ELAN conduit can be performed safely in a wide range of patients with various diagnoses and underlying pathology of the RVOT. The use of homografts in pulmonary position is the best option for the treatment of pulmonary valve/RVOT pathology, however they are not as simple available as the Vascutek RVOT ELAN conduit. Our early results and the easy implantation encourage the use of this conduit. However long-term results have to be assessed in further clinical follow-up studies.

\section{Effects of the Surgical Techniques on Long-Term Results in Patients with Degenerative Mitral Valve Bi-Leaflet Prolapse}

Giuseppe Petrone, Clemente Pascarella, Marianna Buonocore, Angelo Caiazzo, Gianantonio Nappi, Pasquale Santé

Monaldi Hospital-Second University of Naples, Naples, Italy

Background: The aim of the study was to evaluate the long term results in patients with degenerative mitral valve bileaflet prolapse (DMVBLP) undergoing valve repair versus valve replacement comparing the consequences on survival related to each technique. Methods: From 2001 to 2012, 421 patients underwent isolated primary operation for DMVBLP. Valve repair was performed in 146 patients (34.7\%) (MVR group), and valve replacement (MVR) was performed in 275 (65.3\%). MVR patients were divided in 2 subgroups: MVR subgroup A operated preserving the posterior subvalvular apparatus routinely and in selected cases the anterior or both (119 patients, 43.3\%), and MVR subgroup B without preservation of the subvalvular apparatus (156 patients, $56.7 \%)$. Patients in the MVR group were older ( $70 \pm 12$ years vs. $56.4 \pm 14.5)$ compared to those in the MVr group. Results: In MVr group, 5 patients died $(3.4 \%)$ and 6 patients $(4.1 \%)$ underwent MVR due to MVr failure. 11 MVR patients were re-operated (4\%). 11 patients died in subgroup A (9.2\%); 29 patients died in subgroup B (18.6\%). Patients in MVr group demonstrated significant LV end-diastolic diameter (LVEDD) and LV end-systolic diameter (LVESD) decrease postoperatively persistent during follow-up. LV mass also showed a significant regression during the first 4 years maintained during follow-up, while EF showed a trend to improve. In MVR subgroup A, LVEDD was initially decreased but increased during follow-up and LVESD remained high, resulting in a decreasing of EF. In MVR subgroup B, LVEDD and LVESD increased constantly resulting in a worsening of EF. Such data showed a statistical significance. Conclusions: MVr in DMVBLP 
patients achieves better preservation of LV systolic indexes than MVR, due to preservation of the subvalvular apparatus and LV geometry and it guarantees better short and long term survival. Whenever $\mathrm{MVr}$ is unfeasible, we recommend subvalvular preservation when performing MVR, in order to decrease the risk of early and late mortality and to improve LV function.

\title{
Development and Application of Polymer-Matrix Device to Remodel Left Ventricular - Mitral Valve Apparatus in Ischemic Mitral Regurgitation
}

\author{
Akihisa Kataoka, Timothy Tan, Xin Zeng, Michael Garcia, \\ Margo Seybolt, Suzanne Sullivan, Jose Guerrero, \\ Mark Handschumacher, Robert Levine, Judy Hung
}

Massachusetts General Hospital, Boston, MA, USA

Objective: Ischemic mitral regurgitation (IMR) results from mitral valve (MV) tethering by ischemic left ventricular (LV) distortion. We aimed to explore a novel approach to treat IMR by applying a polymer-filled polyester mesh attached to the epicardium of the infarcted myocardium. This poly-mesh can provide direct and adjustable reverse LV remodeling, stabilizing the infarcted wall to reduce IMR. Methods: Eight sheep underwent ligation of circumflex (LCX) branches to produce IMR. Solid polymer granules were enclosed within 2 layers of polymesh. The poly-mesh was attached to infarcted LV and soaked with saline to produce a stable polymer gel of specified volume and compressibility within the mesh. 2D/3D echo and hemodynamic data were obtained (baseline, pre and post poly-mesh), with IMR assessed by $2 \mathrm{D}$ vena contracta (VC). Results: Moderate IMR developed in all sheep post LCX ligation (VC: $0.50 \pm 0.09$ ). After poly-mesh attachment, the MR decreased to trace or mild (VC: $0.50 \pm 0.09$ to $0.26 \pm 0.12 \mathrm{~cm} ; \mathrm{p}<0.01$.

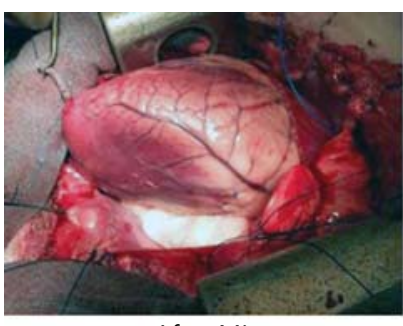

After MI

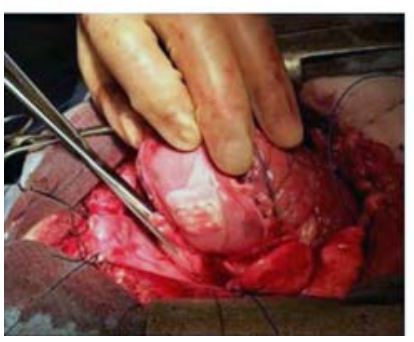

Before bulging

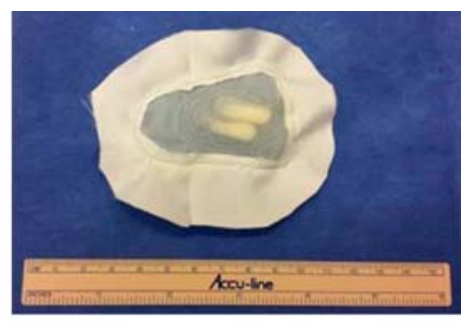

Polymer mesh device

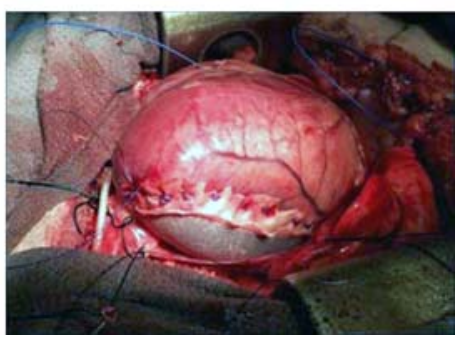

After bulging

Fig. 1. (for Abstract of $\lfloor$ udy Hung).

\section{Non-Linear Optical Visualization Technologies for Characterisation of Aortic Valve Sclerosis in Human and Mouse}

\author{
Anett Jannasch ${ }^{1}$, Saskia Faak ${ }^{1}$, Petra Büttner ${ }^{2}$, Roberta Galli ${ }^{3}$, \\ Christian Schnabel ${ }^{3}$, Edmund Koch ${ }^{3}$, Thomas Waldow ${ }^{1}$ \\ ${ }^{1}$ Heart Center Dresden, Dresden, Germany; ${ }^{2}$ Heart Center \\ Leipzig, Leipzig, Germany; ${ }^{3}$ Institute of clinical Sensoring and \\ Monitoring, Dresden, Germany
}

Objective: Degenerative heart valve disease like aortic valve stenosis (AVS) is characterized by disorganization of fibers in the extracellular matrix and cell invasion. Currently most histological-based methods for characterization of AVS demand for extensive processing of extracted valve material with impact on tissue morphology. We present a novel approach combining coherent anti-Stokes Raman scattering (CARS), endogenous two-photon excited fluorescence (TPEF) and second harmonic generation (SHG) to characterize AVS on native human and murine material. Methods: Native aortic leaflet samples from patients with aortic valve regurgitation (A) and aortic valve stenosis (B) as well as aortic leaflets from apoe ${ }^{-/-}$and wildtype mice were investigated by non-linear optical microscopy (NLO). In addition optical coherence tomography (OCT) enables thickness
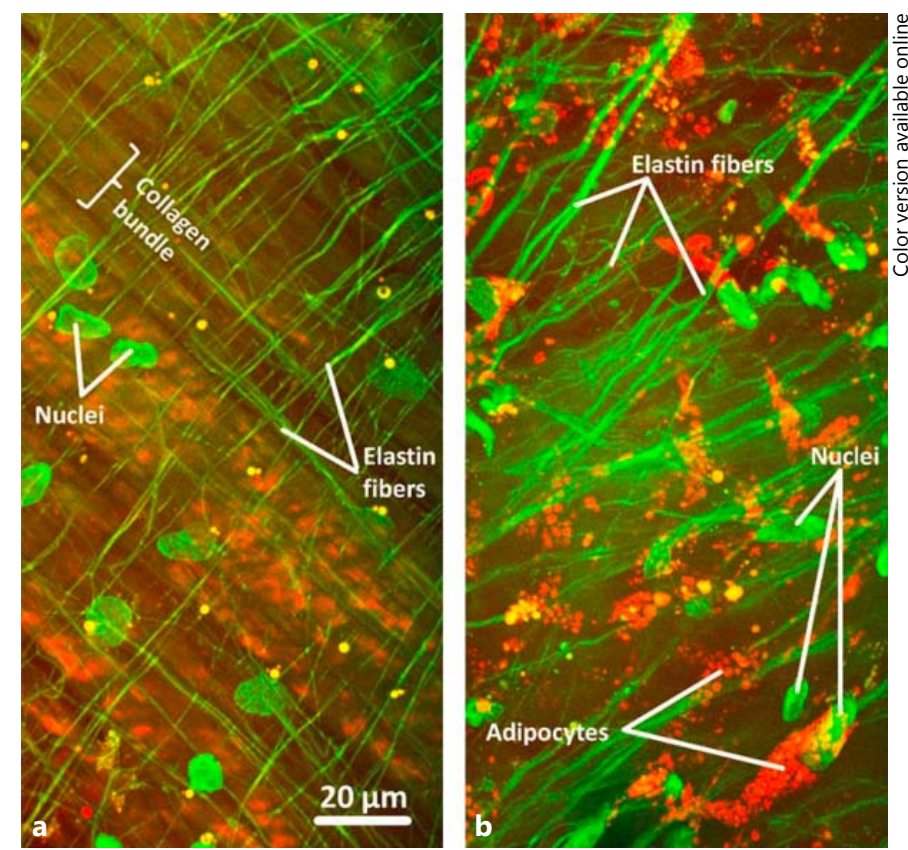

Fig. 1. (for Abstract of Anett Jannasch).

DOI: $10 / 1159 / 000362180$ 
measurements and 3D visualization of murine aortic valve leaflets. Results: TPEF (green) visualized organization degree of collagen and elastin fibers which was higher in leaflet A. CARS (red) gave a strong signal in leaflet B which was attributable to adipocytes located directly above an ossification in the leaflet periphery close to surface. DAPI staining of the nuclei demonstrated the colocalization of lipid droplets and cell bodies. OCT demonstrated increased thickening of valve leaflets in apoe $\mathrm{e}^{-/-}(350 \mu \mathrm{m}$, nodulus region $)$ in comparison to wildtype mice $(200 \mu \mathrm{m}$, nodulus region). TPEF illustrated decreased and fragmented elastin fibers (ventricularis), SHG showed increased and disorganized collagen fibers (fibrosa) and CARS displayed adipocytes infiltration in apoe ${ }^{-/-}$whereas the wildtype did not show any of those pathological changes. Conclusions: Using NLO cusp constituents can be examined simultaneously, three-dimensionally and without extensive manipulation of the sample. These technologies using high resolution imaging methods require no staining procedures, enable the analysis of the progression of AVS and give impressive insights into a complex disease.

\section{Reoperative Mitral Valve Surgery in Patients with Rheumatic Heart Disease}

\section{Jaswinder Singh, Sudeep Singh Sidhu}

Army Hospital (RR), New Delhi, India

Objectives: The aim of this study was to detect the factors for redo surgery and factors that affect late mortality or morbidity after redo mitral valve surgery in patients with rheumatic disease. Methods: A retrospective analysis was carried of all patients undergoing redo mitral valve surgery from January 2001 to October 2013. Results: A total of 303 patients had undergone redo valve surgery during this period. There were 78 patients who had undergone redo mitral valve replacement for rheumatic disease during this period after excluding 247 patients undergoing redo mitral valve surgery after closed mitral. The mean age at the time of operation was $39.4 \pm 12.5$ years (range 11 years to 67 years). Previous mitral valve surgery was open mitral valvotomy in $41(52.56 \%)$ patients, MVR in $34(43.58 \%)$ and mitral valve repair in $3(3.8 \%)$ patients. There were 23 mechanical valve and 11 bioprosthetic valves. Reoperative surgery included $41 \mathrm{MVR}$ and re-MVR in 37 patients. Seventy three mechanical and 5 bioprosthetic valves were used in reoperative surgery. The mean interval to reoperation was $8.9 \pm 4.7$ years ( 3 months to 12 years). Surgical indications were restenosis in 41 , patient prosthesis mismatch in 15 , paravalvular leak in 4 , stuck valve due in 3, endocarditis 2 and structural degeneration of bioprosthetic valve in 10 patients. Operative mortality was $6.4 \%$. Mean follow up was $8.1 \pm 4.6$ years ( 0.4 to 12.5 years). Fifteen patients died during follow up period. Age at surgery, preoperative New York Heart Association (NYHA) class and presence of Left atrial thrombus were found to be independent predictors of late deaths. Higher age, advanced NYHA class, and previous mitral valve replacement were independent predictors of cardiac events. Young age and bioprosthetic valve placement at surgery are independent predictors for redo surgery. Conclusions: Young age and bioprosthetic valve placement at first surgery are independent predictors for redo surgery. Redo mitral valve surgery can be achieved with reasonable early mortality. Long-term results of redo mitral surgery are not necessarily satisfactory in patients with preoperative advanced NYHA class or with a previous mechanical heart valve and left atrial thrombus.

\section{Clinical Outcomes of Patients with Low-Flow, Low-Gradient Severe Aortic Stenosis According to Treatment Modality}

Crochan J. O'Sullivan, Lars Englberger, Stefan Stortecky, Christoph Huber, Nicola Hosek, Ahmed Khattab, Lutz Buellesfeld, Thomas Pilgrim, Bernhard Meier, Stephan Windecker,

Peter Wenaweser

Bern University Hospital, Bern, Switzerland

Objective: We aimed to compare clinical outcomes among patients presenting with 'classical' low-flow, low-gradient severe aortic stenosis according to the assigned treatment modality. Methods: Between April 2005 and December 2012, 210 patients with lowflow, low-gradient severe AS (indexed aortic valve area [AVA] $\leq 0.6 \mathrm{~cm}^{2} \cdot \mathrm{m}^{-2}$, left ventricular ejection fraction [LVEF] $<50 \%$ and mean gradient $(\mathrm{MG})<40 \mathrm{~mm} \mathrm{Hg}$ ) underwent treatment allocation to either medical therapy (MT) $(\mathrm{n}=47)$ surgical aortic valve replacement (SAVR) $(\mathrm{n}=52)$ or transcatheter aortic valve implantation (TAVI) $(\mathrm{n}=111)$. Pre-procedural non-invasive and invasive hemodynamic indices, coronary artery disease (CAD) complexity and procedural characteristics were compared between groups. Primary end-point was all-cause mortality at 1-year. Results: Baseline characteristics were similar between patients allocated to MT and TAVI, whereas SAVR patients were younger (MT $82.47 \pm 5.03$ vs. SAVR $78.43 \pm 54.10$ vs. TAVI $82.04 \pm 5.08$ years, $\mathrm{p}<0.0001)$ and lower risk (STS score MT $10.82 \pm 7.25 \mathrm{vs.SAVR} 4.85 \pm 2.95 \mathrm{vs}$. TAVI7.88 $\pm 4.80 \%$, $\mathrm{p}<0.001)$. CAD complexity was significantly greater among MT patients (SYNTAX score MT 29.18 \pm 17.89 vs. SAVR $20.38 \pm 12.54$ vs. TAVI 21.58 $\pm 14.09, \mathrm{p}=0.036)$. Pre-procedural AVA (MT 0.69 \pm 0.22 , SAVR $0.73 \pm 0.23$, TAVI $\left.0.74 \pm 0.21 \mathrm{~cm}^{2}, \mathrm{p}=0.40\right)$ and MG (MT $25.23 \pm 9.33$ vs. SAVR $29.26 \pm 9.54$ vs. TAVI $28.54 \pm 10.30 \mathrm{~mm} \mathrm{Hg}, \mathrm{p}=$ 0.09 ) were similar between groups, but patients undergoing SAVR had a higher baseline LVEF (MT 30.28 \pm 9.72 vs. SAVR $38.90 \pm 11.94$ vs. TAVI $34.35 \pm 11.32 \%, \mathrm{p}=0.001)$ and lower prevalence of moderate/severe mitral regurgitation (MT 52.3\% vs. SAVR $30.0 \%$ vs. TAVI $52.8 \%, \mathrm{p}=0.02$ ). SAVR patients also had lower pulmonary artery systolic pressures (MT $59.71 \pm 15.29$ vs. SAVR $50.63 \pm 16.15$ vs. TAVI $58.17 \pm 14.72 \mathrm{~mm} \mathrm{Hg}, \mathrm{p}=0.023$ ) on pre-procedural right heart catheterization. Contractile reserve was present in $68.8 \%$ of patients undergoing dobutamine stress echocardiography. At 12-months, the primary endpoint was significantly lower among both SAVR (13.5\% vs. $57.4 \%$, HR $0.17,95 \%$ confidence interval [CI] 0.076-0.40, $\mathrm{p}<0.001)$ and TAVI $(20.7 \%$ vs. $57.4 \%$, HR $0.28,95 \%$ CI $0.16-0.49$, $\mathrm{p}<0.001)$ as compared with MT patients. No significant differences in the primary endpoint were observed between SAVR and TAVI patients $(\mathrm{p}=0.27)$. Conclusions: Among patients with lowflow, low-gradient severe AS, SAVR and TAVI improved survival compared with MT. Clinical outcomes of TAVI and SAVR appeared similar among appropriately selected patients with low-flow, low-gradient severe AS. 


\section{Fresh Decellularized Versus Standard Cryopreserved Pulmonary Allografts for Right Ventricular Outflow Reconstruction During the Ross Operation - Six Years of Follow-Up}

\section{Francisco D. da Costa, Ana Beatriz Brenner Affonso da Costa, Daniele Fornazari, Andrea Dumsch de Aragon Ferreira, Sergio Veiga Lopes, Marcia Olandoski, Tiago Fernandes, Claudinei Colatusso}

Santa Casa de Curitiba PUCPR, Curitiba, Brazil

Objective: Evaluate the mid-term clinical and echocardiographic results of fresh decellularized versus cryopreserved pulmonary allografts for RVOT during the Ross operation. Methods: Between October 2007 and July 2013, 126 Ross operations were performed using a fresh decellularized pulmonary valve allograft. For comparison, we reviewed data from 204 patients from our database that received a cryopreserved allograft. Conduit dysfunction was defined as any peak gradient greater than $40 \mathrm{~mm} \mathrm{Hg}$ or insufficiency grade III or IV. The influence of age, ABO compatibility and $z$-value of the implanted allograft were specifically analyzed. Comparisons were made with Kaplan Meier survival estimate, unbalanced two way repeated measures ANOVA and with linear regression analysis. Results: Peak gradients at hospital discharge was similar between groups, but after the second year of follow-up late gradients were significantly lower for the fresh decellularized group $(p=0.001)$. Moderate or severe regurgitation was present in one case of the decellularized group and in five of the cryopreserved. Freedom from conduit dysfunction at 6 years was significantly better in the decellularized (97\% - CL95\%, 8599\%) versus the cryopreserved (85\% - CL95\%, 78-90\%) group $(\mathrm{p}=0.014)$ at six years. There was no reoperation due to primary valve failure in the decellularized group, while two patients had reoperations for this reason in the cryopreserved group. In addition, decellularization eliminated age, $\mathrm{ABO}$ compatibility and allograft $\mathrm{z}$-score as risk factors for the occurrence of elevated late gradients. Conclusions: Fresh decellularized allografts were superior to conventional cryopreserved allografts for RVOT reconstruction during the Ross operation up to six years of follow-up, with lower late gradients, less pulmonary insufficiency and lower overall incidence of conduit dysfunction.

\section{Various Uses of Sutureless Prostheses: A Single Center Experience}

\section{Giuseppe Santarpino, Ferdinand Vogt, Steffen Pfeiffer, Joachim Sirch, Francesco Pollari, Theodor Fischlein}

Klinikum Nürnberg, Nuremberg, Germany

Objective: Sutureless aortic bioprosthesis bear the potential of easy implantation, reduced ischemic time, and surgical trauma in aortic valve replacement. The aim of the study was to show clinical outcomes in our 4 years experience with patients undergoing sutureless aortic valve replacement with different indications. Methods: Between May 2010 and November 2013, 203 patients (age
$78 \pm 5$ years, female $108-53.2 \%)$ undergoing aortic valve replacement with the sutureless Perceval S bioprosthesis (Sorin Group, Saluggia, Italy) in isolated minimally invasive (111 patients), in REDO (20 patients: 8 with a degenerated aortic bioprosthesis, 12 underwent $\mathrm{CABG}$ ) or in combined procedures (72 patients: 49 CABG, 1 mitral valve replacement, 3 CABG+LAA-closure, 1 1/3 Yacoub, 1 TEA Carotid, 3 Tricuspid valve reconstruction, 1 myomectomie+CABG, 1 aortic ascending replacement+CABG, 1 VSD-Closure, 6 LAA-Closure, 4 Minimaze+LAA closure, 1 myectomie). In-hospital and follow up clinical and echocardiographic data were collected for all patients. Results: The patients received a size S (13), M (77), L (97) or XL (16) prosthesis. In the minimally invasive group, 103 patients received a 'J' sternotomy and 8 a right thoracotomy. Mean logistic European system for cardiac operative risk evaluation was $13 \pm 11.6 \%$, mean aortic cross-clamp time was $44 \pm 17.5$ minutes ( $38 \pm 12.7$ minutes for isolated minimally invasive procedures). In-hospital mortality was $2.5 \%$. We recorded 15 pacemaker implantations (7.4\%). At follow-up (months $20 \pm 11$ ), we observed 6 deaths ( 2 cardiac). Mean transprosthetic gradients were $13.3 \pm 5,13.1 \pm 4.6,13.6 \pm 5,13.2 \pm 3.8 \mathrm{~mm} \mathrm{Hg}$ at 6 months, 1 year, 2 years and 3 years respectively. Conclusions: Implantation of the sutureless Perceval $S$ valve is associated with short cross-clamp time, reducing surgical trauma, and resulting in improved clinical outcome. This self-anchoring valve is a useful tool in many different indications, expecially in an elderly and high risk population.

\section{Perceval S Sutureless Valve Versus Conventional Bioprosthesis: A Matched Case-Control Study}

\section{Sarah Sainte, Herbert De Praetere, Bart Meuris}

KU Leuven, Leuven, Belgium

Objective: The Sorin Perceval $S$ sutureless aortic valve prosthesis shortens aortic cross-clamp (ACC) and cardiopulmonary bypass $(\mathrm{CPB})$ times, thereby possibly reducing morbidity, certainly in elderly or frail patients. There is a lack of comparative studies analyzing outcome of sutureless and conventional valves. We performed a retrospective matched case-control study in 106 patients. Methods: Clinical outcome and hemodynamic data of 53 patients with Perceval S implantation (with or without concomitant CABG) were compared with an age-, gender- and operation type (AVR or $\mathrm{AVR}+\mathrm{CABG}$ ) matched patient cohort receiving an Edwards Perimount valve $(n=53)$. Studied parameters included mortality, CPB and ACC times, transfusion requirements, duration of intubation and length of stay on intensive care and on the regular ward. Laboratory parameters, NYHA class and echocardiographic assessment of valve hemodynamics were obtained at discharge, at 6 months and at 1 year postoperatively. Results: Despite matching, the Perceval patients had a higher Euroscore risk: 12.8 versus 9.7 $(\mathrm{p}=0.01)$. Operative mortality was $0 \%$ in both groups. ACC and $\mathrm{CPB}$ times were significantly shorter in Perceval patients: 24.8 versus $67.3 \mathrm{~min}$ and 56.7 versus $100.0 \mathrm{~min}$ respectively (both $\mathrm{p}<$ $0.0001)$. Perceval patients were on average one hour less in the operating room $(p=0.001)$, they required less transfusion of blood products (packed cells, platelets and plasma), had a shorter inten- 
sive care unit stay, a shorter intubation time $(\mathrm{p}=0.01)$ and a shorter ward stay $(\mathrm{p}=0.03)$. Postoperatively, mean and peak valve gradients were comparable at each time interval for both groups. During follow-up, NYHA status improved significantly and comparably in both groups. Conclusions: In a population of elderly intermediate-risk patients, the shortened cross-clamp times and CPB-times, as obtained with the sutureless Perceval S bioprosthesis, result in a favorable effect on the postoperative recovery and use of resources when compared to a conventional bioprosthesis.

\section{Long Term Results for a Single Centre Aortic Valve Repair Program}

\author{
Andrea Mangini ${ }^{1}$, Monica Contino ${ }^{1}$, Claudia Romagnoni ${ }^{1}$, \\ Massimo Lemma ${ }^{1}$, Guido Gelpi ${ }^{1}$, Paolo Vanelli ${ }^{1}$, \\ Simone Colombo ${ }^{1}$, Carlo Antona ${ }^{2}$ \\ ${ }^{1} \mathrm{~L}$ sacco University Hospital, Milano, Italy; \\ ${ }^{2}$ Università degli Studi di Milano, Milano, Italy
}

Objective: Aortic valvuloplasty seems to be a good alternative to valve replacement for lots of reasons: optimal hemodynamic conditions, absence of prosthetic material, no need of anticoagulation; moreover this kind of surgery allows, in young people, normal aortic annulus growth. Now that aortic valve repair techniques have reached a good standardization level, we want to analyze our medium and long term results in terms of freedom from reoperation and freedom from significant aortic regurgitation, in cases of incompetence due to leaflets and root pathology. Methods: From January 2003 to January 2013 we prospectively identified 235 patients affected by aortic valve regurgitation caused by leaflets pathology (prolapse, fibrosis or retraction) or root dilatation to be treated with a combination of the principal leaflet repair techniques and, when necessary, sparing procedures. Of these, 218 patients were considered eligible in this study. All of them were submitted to: pre and post-operative trans-thoracic echocardiography, pre and post-repair trans-esophageal echocardiography. We standardized our technique dividing the procedure in 4 different moments: valve analysis, leaflets repair, aortic functional unit repair, aortic functional unit stabilization. Follow-up was achieved with periodic echocardiograms and clinical evaluations. Results: 8 patients (3.40\%) died before discharge. Median clinical and echocardiographic follow-up for all patients was 1075.00 [515.251975.25] days. Mean cross clamping time was $101.94 \pm 40.22 \mathrm{~min}-$ utes and mean hospital stay was $10 \pm 6.69$ days. Kaplan-Meier freedom from aortic regurgitation $>2$ and freedom from aortic valve replacement were respectively $92.9 \pm 2.8 \%$ and $94.5 \pm 2.5 \%$ at 9.24 years: 6 patients $(2.75 \%)$ were re-operated with aortic valve replacement for severe aortic regurgitation. We also observed a good effect of aortic surgery on left ventricle: the end-diastolic volume decreased from $137.89 \pm 50.23 \mathrm{ml}$ in the pre-op to $105.17 \pm 31.19 \mathrm{ml}$ at follow-up. Conclusions: Aortic valve leaflet repair seems to be a good and feasible option for selected patients both alone or associated with an aortic sparing technique concerning long-term results. The development of this process was possible thanks to a better comprehension of aortic anatomy and of the pathogenetic mechanisms at the base of the aortic regurgitation.

\section{Single Center 5 Year Results of the St. Jude Medical Trifecta Pericardial Aortic Valve Bioprosthesis with a Sub-Group Analysis of Octogenarians}

\author{
Joseph Bavaria, Rohan Menon, Wilson Szeto, Caroline Komlo, \\ Scott McClure, Sofiane Lazar, Melanie Freas, Nimesh Desai \\ University of Pennsylvania, Philadelphia, PA, USA
}

Objective: The SJM-Trifecta valve is a pericardial bioprosthesis that optimizes hemodynamics with a titanium stent and sewing ring specifically designed for supra-annular placement. We present 285 patient-years of data with this valve; the longest clinical follow-up in the world. We also looked at outcomes of our octogenarians in order to contribute to the ongoing debate of transcatheterAVR (tAVR) vs. openAVR. Methods: At our institution, 100 consecutive SJM-Trifecta valves were implanted from 2007-2009. Perioperative variables were collected on a prospective basis. Clinical follow-up and echocardiograms were obtained at discharge, 6 months, and annually through 5 years. Results: Mean age of patients at time of implant was $74.2 \pm 8.3$ years. $74 \%$ were male, $30 \%$ had a bicuspid aortic valve, $30 \%$ had a history of diabetes and $24 \%$ had previous cardiac surgery. Concomitant CABG was performed in $39 \%$. There was one perioperative mortality (age $=65$, non-valve related), 4 perioperative TIA, zero permanent CVA, and zero patients have moderate or severe AI according to their last echocardiogram. There were no instances of structural valve deterioration or valve-related death through five years. There were two nonstructural explants: one early due to suture compromise of an aberrant intramural left circumflex artery and one late due to endocarditis. Hemodynamic performance, with core lab reviewedechocardiograms revealed an overall mean gradient $(\mathrm{mm} \mathrm{Hg})$ $7.2 \pm 3.7,6.6 \pm 3.1,7.1 \pm 3.4,7.3 \pm 3.9,8.3 \pm 4.0,8.3 \pm 4.5,8.0 \pm 6.7$ at discharge, 6 months, and annually through 5 years respectively. Indexed effective orifice area also remained clinically stable over

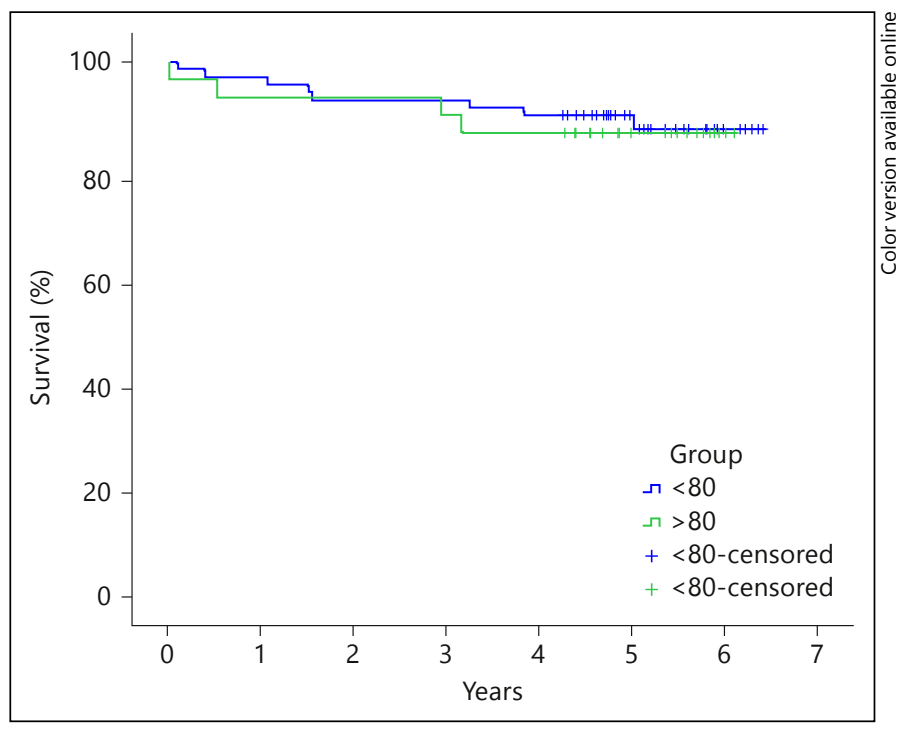

Fig. 1. (for Abstract of Loseph Bavaria). 
time. Among octogenarians $(n=30$, avg STSscore $=7.0), 67 \%$ were female and $73 \%$ had a previous cardiac surgery. Mean echo followup was $2.01 \pm 1.5$ years, and most recent echo reveal zero patients with moderate or severe AI, only 2 patients had severe PPM (IAEOA >0.65), and average mean/peak gradients of $7.7 \pm 4.6 / 14.3 \pm 7.9$ respectively. Survival through five years was comparable to the younger cohort at $87 \%$. Conclusions: The SJMTrifecta valve provides excellent hemodynamics, which remains relatively stable through five years, even in the older $(>80)$, intermediate-risk (STS $>8$ ) population. This data is important as we continue to compare tAVR vs. openAVR technology for intermediate-risk patients.

\section{Physiological Correction of Mitral Valve Regurgitation due to Bileaflet Prolapse Through a 'Portaccess' Totally Video-Guided Approach: Operative Results}

\section{Giovanni Domenico Cresce, Tommaso Hinna Danesi, Loris Salvador}

Cardiac Surgery Division, San Bortolo Hospital, Vicenza, Italy

Objective: Mitral valve regurgitation (MVR) due to bileaflet prolapse (BP) is one of the most complex lesion to be treated by reconstructive surgery. Aim of this study was to report our operative results in the management of BP-MVR using a physiological repair through a minimally invasive totally video-guided approach. Methods: From January 2010 to November 2013, 601 patients underwent Totally Video-Guided Port-Access Cardiac Surgery at our Institution. Among them, 506 (84\%) received a mitral valve repair. A degenerative pathology underlying a severe mitral regurgitation was the indication for surgery in 409 cases; among them $78(19 \%)$ patients ( 50 males; mean age $59.4 \pm 16.1$ years) had a BP-MVR. Fourteen patients (17.8\%) were in NYHA functional class III or IV. The technique utilized to repair the MVR was the e-PTFE neo-chordae implantation completed with a posterior annuloplasty using a flexible band. Associated procedures were: tricuspid valvuloplasty in 4 cases (5\%), AFib radiofrequency ablation in 14 pts (18\%) and a PFO closure in 34 pts (43.5\%). Results: Mean CBP time and aortic cross-clamp time were $148 \pm 53$ and $107 \pm 52$ min respectively. There was no in-hospital mortality. We had no conversion to conventional sternotomy, reoperation for bleeding and aortic dissection. We achieved the mitral valve repair in all cases. Six patients $(7.6 \%)$ required an edge-to-edge stitch to avoid systolic anterior motion (SAM) of the anterior MV leaflet. Postoperative complications were: new atrial fibrillation onset in 6 patients (7.6\%), atrio-ventricular block requiring permanent pacing in 2 patients (2.5\%). No major neurological events were observed. At discharge postoperative echocardiogram showed mild residual MVR in $2(2.5 \%)$ cases, due to mild SAM without left ventricular outflow obstruction. Conclusions: Our results showed the effectiveness of the respect-than-resect technique in the treatment of MVR due to BP. This technique is particularly useful with a completely video-guided thoracoscopic MV approach.

\section{Tricuspid Annuloplasty Using the MC3 Ring for Functional Tricuspid Regurgitation: An Analysis of Midterm Outcomes}

\author{
Yeow Leng Chua, Philip Y.K. Pang, Mingjie Huang, Noah Tapaua
}

National Heart Centre Singapore, Singapore, Singapore

Objective: The aim of this study was to investigate the clinical and echocardiographic outcomes after tricuspid annuloplasty with the 3-dimensional Edwards Lifesciences MC3 ring. Methods: A retrospective review was conducted for 120 consecutive patients who underwent tricuspid annuloplasty for functional tricuspid regurgitation (TR) between 2003 and 2013. Results: Mean age was $60.1 \pm 12.1$ years with $57.5 \%$ females. One hundred and seven patients $(89.2 \%)$ underwent mitral valve surgery (repair $[n=67]$, replacement $[\mathrm{n}=40]), 10(8.3 \%)$ underwent mitral and aortic valve replacement, $2(1.7 \%)$ underwent repair of atrial septal defect and $1(0.8 \%)$ underwent aortic valve replacement. The indication for tricuspid annuloplasty was severe (4+) or moderately severe (3+) TR in 53 patients $(44.2 \%)$ and moderate $(2+)$ or less TR with tricuspid annular dilatation in 67 (55.8\%). The mean preoperative TR grade was $2.6 \pm 1.0$ and the mean preoperative pulmonary artery systolic pressure (PASP) was $59.0 \pm 21.2 \mathrm{~mm} \mathrm{Hg}$. The average preoperative tricuspid annulus diameter and implanted MC3 ring size were $33.9 \pm 5.5 \mathrm{~mm}$ and $26.8 \pm 1.4 \mathrm{~mm}$ respectively. The mean follow-up period was $4.2 \pm 2.9(0.2-10.1)$ years. Two patients $(1.7 \%)$ died from multi-organ failure within 30 days of surgery. No patients developed MC3 ring-related complications such as atrioventricular block, ring dehiscence or tricuspid stenosis. Echocardiography after a mean of $3.7 \pm 2.6(0.3-9.5)$ years showed a significant decrease in the average TR grade $(2.6 \pm 1.0$ to $0.7 \pm 0.5, \mathrm{p}<0.001)$, PASP $(59.0 \pm 21.2$ vs. $32.1 \pm 10.2 \mathrm{~mm} \mathrm{Hg}, \mathrm{p}<0.001)$ and tricuspid annulus diameter $(33.9 \pm 5.5 \mathrm{~mm}$ vs. $26.2 \pm 5.1 \mathrm{~mm}, \mathrm{p}<0.001)$. Overall survival rates were $97.4 \pm 1.5 \%, 91.8 \pm 3.1 \%$ and $88.0 \pm 4.8 \%$ at 1,5 and 8 years respectively. Freedom from recurrent moderate or severe TR was $98.9 \pm 1.1 \%$ and $95.5 \pm 2.6 \%$ at 1 and 5 years respectively. Multivariate analysis revealed that recurrent moderate or severe TR was significantly associated with elevated PASP. Conclusions: Tricuspid annuloplasty using the MC3 ring corrects functional TR effectively and can be performed with a low incidence of mortality and morbidity, with excellent midterm clinical and echocardiographic results.

\section{Incidence and Progression of Mild Aortic Regurgitation After the David I Procedure}

\author{
Fabian A. Kari ${ }^{1}$, Sami Kueri ${ }^{1}$, Bartosz Rylski ${ }^{1}$, Maximilian Russe ${ }^{2}$, \\ Matthias Siepe ${ }^{1}$, Friedhelm Beyersdorf ${ }^{1}$ \\ ${ }^{1}$ Heart Center, University of Freiburg, Freiburg, Germany; \\ ${ }^{2}$ University of Freiburg, Freiburg, Germany
}

Objective: Mild aortic regurgitation (AR) and progression of AR might be a significant hazard after David I valve-sparing aortic root replacement (V-SARR), potentially resulting in reoperation. Methods: Between 2003 and 2013, $n=167$ patients ( $n=116$ male, 
age $47 \pm 19$ years) underwent the David I procedure ( $\mathrm{n}=157$ aortic root and/or ascending aortic aneurysm, $\mathrm{n}=10$ Type A dissection, $\mathrm{n}=53$ Marfan). Mid-term follow-up TEE (mean 449 days, 741,427 days) was analyzed. Median preoperative AR was $1+$ to $2+$, $\mathrm{n}=58$ patients $(35 \%)$ had preoperative $\mathrm{AI} \leq 1+$ and $\mathrm{n}=12$ patients (12\%) preoperative $\mathrm{AR} \geq 3+$, while no patients with $\mathrm{AR}>3+$ were elected for David procedure. Uni- and multivariate analysis and LogRank tests were calculated. Valvular endpoints were freedom from AR 2+, freedom from AR 1+ and freedom from new AR or AR progression. Results: AR was reduced from a preoperative median of $1-2+$ to a median of $0-1+$ before discharge $(\mathrm{p}<0.001)$. Freedom from AR 2+ was $78 \%$ and from AR progression $60 \%$ at 2 years. Most patients progressed subclinically from no to trivial or minimal AR. Severe AR $>3+$ occurred in 4 patients, leading to reoperation and mechanical valve replacement in three cases. Aneurysm size and aortic downsizing were not linked to higher incidence of mild AR (all p > 0.05). Conclusions: The incidence of subclinical, progressing AR is significant after the David procedure. While higher grade $\mathrm{AR}$ results in the need of reoperation almost invariably, the role of subclinically progressing low grade AR has to be characterized in future follow-up studies.

\section{Aortic Root/Valve Management in Patients with Connective Tissue Syndromes - Is Limited Resection Justified?}

\author{
Fabian A. Kari ${ }^{1}$, Elizabeth H. Stephens ${ }^{2}$, Bartosz Rylski ${ }^{1}$, \\ Maximilian Russe ${ }^{3}$, Matthias Siepe ${ }^{1}$, Friedhelm Beyersdorf ${ }^{1}$ \\ ${ }^{1}$ Heart Center, University of Freiburg, Freiburg, Germany; \\ ${ }^{2}$ Columbia University New York, New York City, USA; NY, USA; \\ ${ }^{3}$ University of Freiburg, Freiburg, Germany
}

Objectives: The ideal treatment of the aortic root and valve in patients with connective tissue syndromes presenting with aortic root aneurysm or aortic dissection remains subject to debate. Methods: Prospective collection of clinical and functional followup data was performed at our institution's connective tissue syndrome-dedicated clinic. Kaplan Meier, LogRank calculations and the Cox proportional Hazards Model were used to identify differences between treatment groups with respect to type of aortic root and valve management. Results: 203 patients with confirmed Marfan-Syndrome (MFS 1, n = 189), Loeys Dietz Syndrome $(n=9)$ or Familial Thoracic Aortic Dissection $(n=2)$ were followed clinically. Cumulative clinical follow-up comprised 1028 patient-years. Over a mean follow-up period of $8.1 \pm 7.6$ years $n=130(65 \%)$ patients were in need of an elective aortic aneurysm repair $(n=85$, $65 \%)$ or an emergency $(n=45,35 \%)$ aortic procedure for aortic aneurysm or acute/subacute aortic dissection. $\mathrm{N}=55$ (42\%) underwent a composite valve graft procedure, $\mathrm{n}=58(45 \%)$ a David valve-sparing aortic root replacement procedure $(\mathrm{n}=7 \mathrm{mod}$. David II, $\mathrm{n}=51$ David I). In $\mathrm{n}=17(13 \%)$ patients the aortic root was not fully replaced during the initial procedure. This was done more often in the setting of a dissection, with the root either being unaffected by the dissection or resuspended. An untreated aortic root at the time of primary procedure resulted in freedom from reoperation of $34 \%(9-61 \%)$ at 15 years vs. $87 \%$ (74-92\%) for a proce- dure including root replacement $(\mathrm{p}=0.039)$. Freedom from root or valve reoperation after the David procedure was 95\% (79-99\%) and $88 \%(65-96 \%)$ at 4 and 8 years. Conclusions: Prophylactic surgery using a valve-sparing technique can be considered the best root treatment for connective tissue disorders currently available. Aortic root replacement should be performed during acute aortic dissection repair if a connective tissue disorder is known or likely even in the setting of a non-dissected root.

\section{Focus on Surgical TAVR: Impact on Complications on Short- and Midterm-Outcome and Implications for Preoperative Risk Assesment}

\author{
Maximilian Scherner Jr., Navid Madershahian, Svenjy Ney, \\ Kathrin Kuhr, Tanja Rudolph, Yeong Hoon Choi, Elmar Kuhn, \\ Thorsten Wahlers \\ University Hospital of Cologne, Cologne, Germany
}

Objective: We investigated complications after surgical TAVR, analyzed the impact of specific complications on 30-day and midterm-outcome (VARC II Endpoints) and developed a risk-stratification-model to determine the impact of relevant comorbidities. Methods: 195 patients underwent transapical (TA) or transaortic (Tao)-AVR between January 2009 and December 2012. The prospectively collected data were used to perform elaborate uni- and multivariate risk factor analysis as well as survival analysis. Results: 30 -day mortality was $12.3 \%$, most frequent periprocedural complications were acute kidney Injury (41\%), bleeding (12.8\%), intraoperative CPB-use (9.7\%) and intraoperative CPR (9.2\%). COPD (OR 2.76.95\%, CI (1.37-5.57); $\mathrm{p}=0.005)$, prior myocardial infarction (OR $2.24(0.97-5.17) ; \mathrm{p}=0.059)$ and logistic EuroSCORE $>20 \%$ (OR $2.54(1.29-4.99) ; \mathrm{p}=0.007)$ were independent multivariable predictors for diminished 30-day-safety, whereas preoperative hemoglobin $<12 \mathrm{mg} / \mathrm{dl}$ (OR 2.34 (1.10-4.98)) and logistic EuroSCORE $>20 \%$ (OR 2.74 (1.23-6.07)) were predictors for diminished 1 year survival. Complications (i.e. intraoperative cardiopulmonary resuscitation, cardiopulmonary bypass use, Pneumonia, SIRS, Stroke, catecholamine-support) were analyzed in separate models and adjusted for preoperative factors to determine their impact on 30-day and 1-year outcome. Aditionally, we calculated a risk-stratification-model based on the results of the multivariable analysis to allow a preoperative assessment of 30-day and one-year outcome in dependency on preoperative comorbidities. Conclusion: Surgical-TAVR patients represent a specific entity in terms of preoperative factors and perioperative complications. Our analysis allows the assessment of the impact of preoperative and perioperative factors on the outcome after surgical-TAVR as long as a specific TAVR-Score is missing. 


\section{Surgical Presentation, Cardiac Remodeling and Long-Term Outcome of Organic Mitral Regurgitation According to Gender}

\section{Francesca Mantovani, Marie-Annik Clavel, Rakesh M. Suri, Maurice Enriquez-Sarano}

Mayo Clinic, Rochester, MN, USA

Objective: Guidelines for management of mitral regurgitation (MR) do not provide sex-specific MR severity grading, cardiac remodeling assessment or surgical indications. Guidelines do not suggest gender differences in presentation and outcome of MR surgery but recent data suggest lower repair rate and long-term survival in women. Methods: In 201 women and 454 men with 1) mitral surgery for organic MR (1990-2000), 2) comprehensive pre-operative MR quantification, and 3) pre and post-operative echocardiographic assessment of cardiac remodeling, long-term follow-up was analyzed. Results: Pre-operatively women vs. men had similar age and ejection fraction but smaller left ventricular $(\mathrm{LV})$ diastolic diameter $(56 \pm 6$ vs. $60 \pm 7, \mathrm{p}<0.0001)$ more often labeled normal size ( 21 vs. $13 \%, \mathrm{p}=0.0007$ ) and smaller LV systolic and left atrium size (all $\mathrm{p}<0.0001)$. Women had smaller quantified regurgitant volume $(82 \pm 32$ vs. $98 \pm 46 \mathrm{~mL} /$ beat, $\mathrm{p}<0.0001)$ but higher pulmonary pressure $(\mathrm{p}<0.0001)$ more heart failure symptoms (37 vs. $19 \%, \mathrm{p}=0.01$ ), which more often triggered surgery $(\mathrm{p}=0.02)$. Normalizing for body size, LV diastolic diameter $(33 \pm 5$ vs. $\left.30 \pm 4 \mathrm{~mm} / \mathrm{m}^{2}, \mathrm{p}=0.19\right)$ demonstrating similar reduction of large volume overload by surgery. Valve repair (91\%) was lower in women but similar in repairable valves (degenerative or endocarditic MR, both $\mathrm{p}>0.56)$. During follow-up (9.8 \pm 4 .1 years) women vs. men had similar survival (15-year $59 \pm 5$ vs. $59 \pm 3 \%, \mathrm{p}=0.6)$ but more frequent heart failure (15-year $36 \pm 7$ vs. $19 \pm 3 \%, \mathrm{p}=0.02$; adjusted hazard-ratio $1.63[1.06-2.48], \mathrm{p}=0.03$ ) linked to the more frequent pre-operative heart failure symptoms $(\mathrm{p}<0.001)$. Conclusions: Women underwent surgery for organic MR with more advanced clinical presentation but with smaller absolute LV dimensions and lower regurgitant volume, suggestive of underestimation of MR severity in women, demonstrated by profound postoperative reverse remodeling. Excess postoperative heart failure linked to worse preoperative presentation also points to late surgical referral. Valve repair similarly achieved in repairable lesions and similar long-term survival in women and men suggest that women should not be denied early surgery for organic MR.

\section{Effect of Mitral Annuloplasty Ring Shape and Size on Leaflet and Myofiber Stress Following Repair of Posterior Leaflet Prolapse: A Patient-Specific Finite-Element Simulation}

\author{
William G. Morrel, IV ' Liang Ge ${ }^{1}$, Alison Ward ${ }^{2}$, Zhihong Zhang ${ }^{3}$, \\ Joe Pantoja', Sarthak Gulati ${ }^{4}$, Eugene A. Grossi', \\ Mark B. Ratcliffe ${ }^{1}$ \\ ${ }^{1}$ University of California, San Francisco, San Francisco, CA, \\ USA $;{ }^{2}$ NYU School of Medicine, New York, NY, USA; ${ }^{3}$ San \\ Francisco Veterans Affairs Medical Center, San Francisco, CA, \\ USA; ${ }^{4}$ University of California, Los Angeles, Los Angeles, CA, \\ USA
}

Objective: Calculate changes in leaflet coaptation and stresses on mitral valve (MV) and left ventricle (LV) resulting from $\mathrm{MV}$ repair in a patient with posterior leaflet (P2) prolapse degenerative mitral regurgitation (MR). Analyze three mitral annuloplasty (MA) devices, each in four sizes, to assess impact of ring shape and size on leaflet and LV stress. Methods: Magnetic resonance imaging (MRI) was performed before and intra-operative 3D trans-esophageal echocardiogram (TEE) was performed before and after repair of P2 prolapse in a single patient. Repair included triangular resection and placement of a CG Future partial annuloplasty band. MRI and TEE images were co-registered to create a $3 \mathrm{D}$ finite-element model. Elements of the P2 region were removed to model leaflet resection, and virtual sutures were used to repair the leaflet and attach the MA ring. The model was optimized to match leaflet coaptation, end-diastolic volume, and end-systolic volume from the imaging data. Two additional rings were digitized using microCT. Simulations were completed in four sizes for each ring. Anterior and posterior leaflet and LV myofiber stress were compared. Results: All three rings abol-

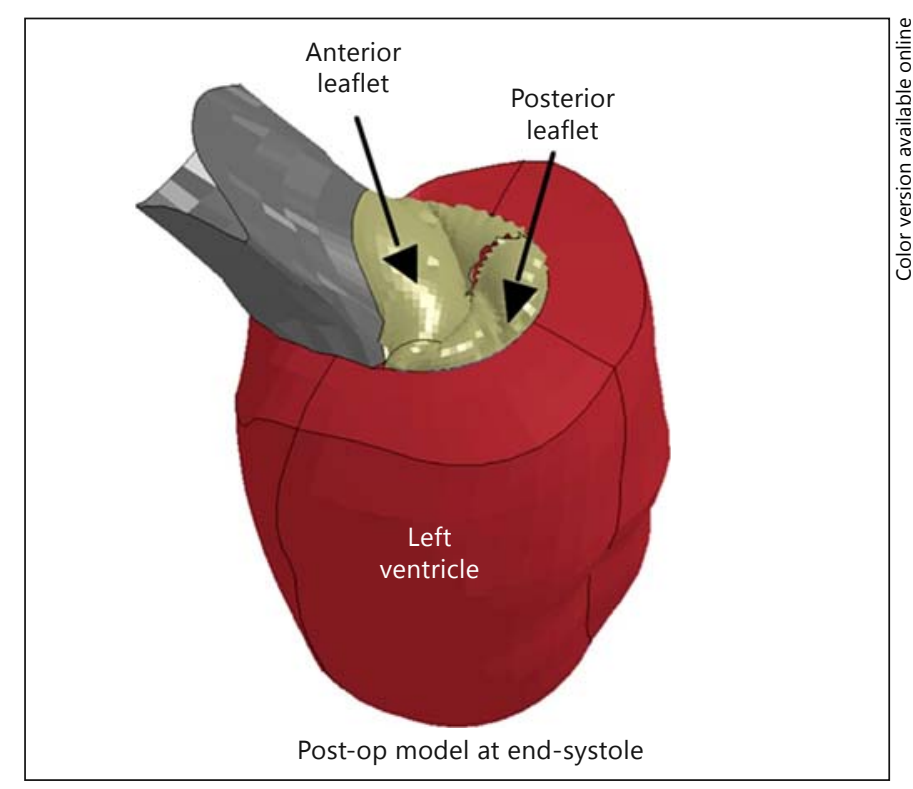

Fig. 1. (for Abstract of William G. Morrel). 
ished regurgitation. Repair decreased stress in the anterior leaflet and LV wall during diastole and systole but increased posterior leaflet stress. Compared to a flat ring, saddle-shape increased anterior leaflet stress while decreasing posterior leaflet and myofiber stress at end-diastole. At end-systole, saddle-shape decreased leaflet and myofiber stress modestly. As ring size decreased, leaflet and myofiber stress and orifice area decreased regardless of ring shape. Undersizing the ring by two sizes decreased anterior leaflet stress by $12.4 \pm 5.8 \%$ and posterior leaflet stress by $14.1 \pm 3.0 \%$ (averaged across all ring shapes). Conclusions: None of the studied ring shapes was superior in all categories. Undersizing was associated with decreased end-systolic anterior leaflet, posterior leaflet, and myofiber stress and also decreased orifice area. Creation of additional patient-specific models will allow broader clinical conclusions. This modeling method may allow calculation of optimal ring shape and creation of a virtual surgical training tool.

\section{Transapical Aortic Implantation of Transcatheter Tissue Engineered Heart Valves Using a Self-Expanding Anatomically Orienting System}

\author{
Maximilian Y. Emmert, Benedikt Weber, Volkmar Falk, \\ Simon P. Hoerstrup
}

University of Zurich, Zurich, Switzerland

Objective: Although transcatheter aortic-valve implantation (TAVI) has proven to be a valid alternative for the treatment of aortic valve disease in high-risk patients, the currently utilized prostheses are well known to present with structural degeneration over time. The concept of tissue-engineered heart-valves (TEHV) with remodelling and repair capacities has been suggested as a promising alternative to overcome these limitations. Here we report our initial experience to merge the concept of TEHV with transapical, anatomically orienting delivery system. Methods: Tri-leaflet TEHV fabricated from biodegradable syntheticscaffolds were sewn onto self-expanding nitinol stents before they were seeded with autologous bone marrow derived mononuclear cells. Thereafter, they were transapically implanted into adult sheep using the JenaValve transapical TAVI System (JenaValve, Munich/Germany). The TEHV were assessed with angiography and echocardiography before they were harvested to undergo post mortem analysis. Results: Anatomically-oriented transapical implantation of bone marrow mononuclear cell derived TEHV into the orthotopic aortic-valve position was successful in all animals. Post implantation angiography and echocardiography demonstrated appropriate positioning in the aortic root fully excluding the native leaflets without any signs of coronary occlusion. The TEHV were competent, had a low mean gradient and displayed intact leaflets well accepting the aortic pressure loading. Minor aortic regurgitation and/or paravalvular leakage was detectable in selected animals. Post-mortem computed tomography confirmed optimal positioning of the TEHV showing the JenaValve stent to be well integrated into the aortic root. Gross examination of the explanted TEHV showed intact leaflets with well identifiable cusps, without any signs of leaflet thicken- ing or shortening. Conclusions: In this study we demonstrate the feasibility to merge the concept of TEHV with transapical delivery using a state-of-the-art, anatomically orienting system. These data represent a further milestone towards a translational, transcatheter-based TEHV approach.

\section{A New Feature in Myxomatous Mitral Valve Disease: Cleft-Like Indentation - Prevalence, Mechanisms and Implication}

\author{
Francesca Mantovani ${ }^{1}$, Marie-Annik Clavel ${ }^{1}$, Ori Vatury' ${ }^{1}$ \\ Rakesh M. suri' ${ }^{1}$, Sunil mankad ${ }^{1}$, Josef F. Malouf', \\ Hector I. Michelena ${ }^{1}$, Luigi P. Badano ${ }^{2}$, Maurice Enriquez-Sarano ${ }^{1}$ \\ ${ }^{1}$ Mayo Clinic, Rochester, MN, USA; \\ ${ }^{2}$ Università degli Studi di Padova, Padova, Italy
}

Background: Cleft-like indentations of posterior leaflet (CLI) were recently described in Myxomatous mitral valve disease (MVD). However CLI prevalence, mechanisms and implications are unknown. Aims: To investigate 3D-echocardiographic prevalence and mechanisms of CLI in MVD and their implications. Methods: Real-time 3-dimensional transesophageal echocardiography (3D TEE) of the mitral valve was acquired in 49 patients with MVD and severe regurgitation prior to mitral valve repair surgery. CLI was defined echocardiographically as a visible defect seen during systole, extending at least one half of the depth of the adjacent mitral leaflet. 3D prevalence of CLI was compared with surgeon visual inspection. Mitral annular (anteroposterior and intercommissural diameters, area) and leaflets dimensions (3-dimensional area, volume of prolapse) were measured in end-systole with dedicated quantification software. Results: CLI in the posterior leaflet was identified with 3D TEE in 17 (35\%) patients. Among them, 15 $(88 \%)$ were confirmed by surgical visual inspection. Compared to patients without CLI $(\mathrm{n}=32)$, those with CLI $(\mathrm{n}=17)$ had similar left ventricle end-diastolic diameter ( $57 \pm 6$ vs. $57 \pm 6 \mathrm{~mm}$; $\mathrm{p}=0.91$ ) and severity of mitral regurgitation (regurgitant volume $89 \pm 56$ vs. $79 \pm 29 ; \mathrm{p}=0.49)$. However, patients with CLI had a smaller mitral annulus as documented by antero-posterior diameter (42.2 $\pm 7.1 \mathrm{vs.}$ $47.0 \pm 7.5 \mathrm{~mm}, \mathrm{p}=0.04)$, intercommissural diameter ( $36.5 \pm 4.5$ vs. $41.4 \pm 5.9 \mathrm{~mm}, \mathrm{p}=0.005)$ and area $\left(1289 \pm 326\right.$ vs. $1619 \pm 427 \mathrm{~mm}^{2}$, $\mathrm{p}=0.008)$ compared to patients without CLI. There was also a trend toward a smaller volume of prolapse in patients with CLI $(1.9 \pm 1.2$ vs. $4.0 \pm 4.3 \mathrm{ml}, \mathrm{p}=0.06)$ compared to patients without CLI. Importantly, the global quantity of valve tissue was markedly less in patients with than without CLI (leaflets area $1574 \pm 409$ vs. $\left.2019 \pm 652 \mathrm{~mm}^{2}, \mathrm{p}=0.01\right)$, especially due to a smaller posterior leaflet area $(783 \pm 254$ vs. $1048 \pm 409, \mathrm{p}=0.02)$. The closure of all CLI was required during surgical valve repair. Conclusions: Cleft-like indentations of the posterior leaflet are often present in MVP and are identified purely by 3DTEE, with high accuracy. CLI occur in the context of decrease amount of leaflet tissue especially of the posterior leaflet. Indeed, CLI are unrelated to annular enlargement or excess of tissue. Identification of CLI is important as these defects may require surgical closure. 


\section{Posterior Papillary Muscle Relocation Affects Stress and Pump Function: Finite Element Based Surgery}

Joe L. Pantoja' ${ }^{1}$ Liang Ge ${ }^{1}$, Zhihong Zhang ${ }^{2}$, William G. Morrel' ${ }^{1}$, Sarthak Gulati ${ }^{3}$, Eugene A. Grossi ${ }^{4}$, Mark B. Ratcliffe ${ }^{1}$

${ }^{1}$ University of California San Francisco, San Francisco, CA, USA; ${ }^{2}$ Veterans Affairs Medical Center, San Francisco, CA, USA;

${ }^{3}$ University of California Los Angeles, Los Angeles, CA, USA;

${ }^{4}$ New York University, New York City, NY, USA

Objective: The role of posterior papillary muscle relocation (PPM:PPMR) concomitant to mitral annuloplasty (MA) in the management of chronic ischemic functional mitral regurgitation (CIMR) is controversial. Traction suture placement and PPM displacement vary between procedures. The goal of this study was to determine the effects of PPM displacement and various anchor points on left ventricular (LV) regional myofiber stress and pump function. Methods: Previously described finite element (FE) models of the ovine LV, 16 weeks after postero-lateral myocardial infarction (MI) were used. PPMR + true-sized MA was simulated using the virtual suture technique (figure 1). PPMR anchor points tested included the mitral commissures and mid-anterior annulus. The anchor point to PPM distance (APD) was reduced between $10 \%-40 \%$ of baseline distance. In each case, myofiber stress in the MI, border zone (BZ), and remote zone, ventricular short axes, coaptation depth, and stroke volume were calculated. Outcomes were analyzed using mixed-model linear regression. Results: PPMR reduced end-diastolic and end-systolic myofiber stress proportionally to the reduction in APD in all LV regions (all regions: $\mathrm{p}<0.01)$. Stress reduction was greatest in the MI $(8.67 \pm 1.31 \mathrm{kPa})$ and least in the remote zone $(1.03 \pm 0.12 \mathrm{kPa})$. When considering ventricular shape, reductions in septal-lateral short axis had a strong effect on stress reductions in all regions (all regions: $\mathrm{p}<$ 0.01 ) while the effect of APD reduction become insignificant (MI: $\mathrm{p}=0.82$, BZ: $\mathrm{p}=0.99$, Remote: $\mathrm{p}=0.37)$. Despite decreases in stroke volume at $40 \%$ APD reductions, forward stroke volume increased in all PPMR cases. Finally, coaptation depth decreased with higher APD reductions. Conclusions: These findings support the addition of ventricular reshaping, subvalvular procedures to MA in the management of CIMR. When selecting an anchor point and post-surgical PPM position, surgeons should consider postsurgical ventricular shape as it mediates stress reduction. In the future, FE optimized surgical procedures implemented on patient specific LV models may elucidate the impact of myofiber stress reduction on ventricular remodeling.

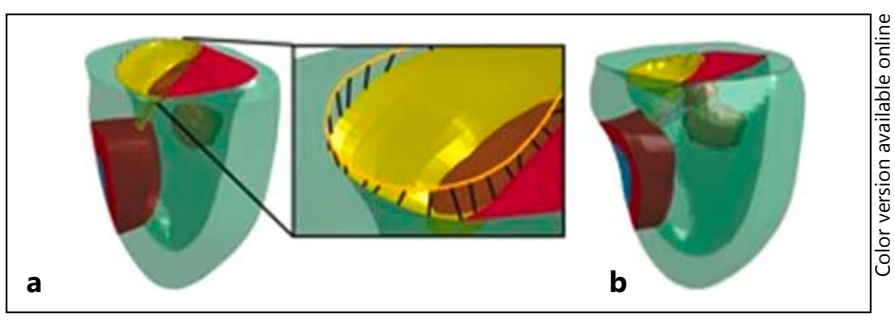

Fig. 1. $L V$ with $P P M R+M R$ prior to (a) and after tightening sutures (b) (for Abstract of Joe L. Pantoja).

\section{Long-Term Follow-Up of the Edwards Pericardial Aortic Valve: Is It the Standard with Which TAVI Should Be Compared?}

\author{
Alfredo Trento, Michele A. De Robertis, James Mirocha, \\ Wen Cheng, Morcos Awad, Andrea Ruzza, Lawrence S.C. Czer \\ Cedars-Sinai Medical Center, Los Angeles, CA, USA
}

Objective: With the advent of transcutaneous tissue aortic valve implantation we sought to review the outcomes of surgical tissue aortic valve replacement to understand what patient characteristics influence survival. Methods: From November 1992 through December 2012, a total of 2,069 patients underwent aortic valve replacement (AVR) surgery with an Edwards bovine pericardial prosthesis, Perimount $(\mathrm{n}=900,43.5 \%)$ or Magna $(\mathrm{n}=$ $1169,56.5 \%$ ). Patients were followed for 1 to 21 years (median 9.9 years). Survival was analyzed using multivariable Cox models. Results: We report results for 2 age strata: younger (60 to 79 years) and older ( 80 to 89 years). Median ( $95 \% \mathrm{CI}$ ) survival in the younger group was $12.8(11.3-13.4)$ years and $6.1(5.8-6.7)$ years in the older group. Important patient characteristics for overall survival were age, gender, bicuspid valve (BAV), coronary artery disease (CAD), left ventricular ejection fraction (LVEF) and patientprosthesis mismatch. By a Cox multivariate model, in the younger strata $(n=1,073)$, the following variables were associated with worse survival: lower LVEF (for a 5\% decrease, HR $=1.07,95 \%$ $\mathrm{CI}=1.03-1.10, \mathrm{p}=0.0008)$, prior cardiac surgery $(\mathrm{HR}=1.40,95 \%$ $\mathrm{CI}=1.09-1.80, \mathrm{p}=0.009)$, patient prosthesis mismatch $(\mathrm{HR}=$ $1.32,95 \% \mathrm{CI}=1.06-1.64, \mathrm{p}=0.014)$, diabetes $(\mathrm{HR}=1.37,95 \%$ $\mathrm{CI}=1.09-1.72, \mathrm{p}=0.008) . \mathrm{BAV}$ was protective $(\mathrm{HR}=0.58,95 \%$ $\mathrm{CI}=0.44-0.76, \mathrm{p}=0.0001)$. However, for the older patients who underwent AVR in their 80s $(\mathrm{n}=658)$, only CAD $(\mathrm{HR}=1.30,95 \%$ $\mathrm{CI}=1.01-1.67, \mathrm{p}=0.041)$ and patient prosthesis mismatch $(\mathrm{HR}=$ $1.42,95 \% \mathrm{CI}=1.16-1.75, \mathrm{p}=0.0007)$ were associated with worse survival. EF was borderline (for a 5\% decrease, $\mathrm{HR}=1.04,95 \%$ $\mathrm{CI}=1.00-1.08, \mathrm{p}=0.051)$. Prior cardiac surgery was not significant $(\mathrm{HR}=1.04,95 \% \mathrm{CI}=0.79-1.37, \mathrm{p}=0.79)$. Conclusions: The predictors of survival by multivariate Cox analysis vary in different age strata. This suggests that different factors may influence survival in younger and older patients. These considerations should be taken into account when examining data in TAVI patients as well as surgical AVR.

\section{Long Term Outcomes and Quality of Life in Octogenarians After Complex Valve Surgery}

\author{
Kumaresan Nagarajan, Asterinos Chnaris, Muhammed Israr, \\ Parminderjit Jayia, Sophia Mahboob, Alan Neville, \\ Heyman Luckraz
}

Royal Wolverhampton Hospital NHS Trust, Wolverhampton, United Kingdom

Objective: To assess long term outcomes and quality of life in octogenarians after complex valve surgery as compared to isolated valve surgery in the same age group. Methods: Data was 
Table 1. (for Abstract of Kumaresan Nagarajan)

\begin{tabular}{lccc}
\hline Parameters & $\begin{array}{l}\text { Complex } \\
\text { Surgery } \\
\text { Group }\end{array}$ & $\begin{array}{l}\text { Isolated } \\
\text { Valve } \\
\text { Group }\end{array}$ & p-value \\
\hline Mean-log euroscore*** & 16.3 & 12.1 & 0.01 \\
30 day mortality & $11.5 \%$ & $4.1 \%$ & 0.05 \\
Late mortality & $25.3 \%$ & $17.6 \%$ & 0.2 \\
In hospital stay** (days) & 10 & 7.5 & $<0.01$ \\
Re-exploration & $12.1 \%$ & $8.1 \%$ & 0.6 \\
ICU* readmission & $7.1 \%$ & $6.8 \%$ & 0.9 \\
Chest infection & $18.6 \%$ & $12.7 \%$ & 0.3 \\
Cerebrovascular event & $1.7 \%$ & $1.4 \%$ & 0.6 \\
Gastro-intestinal complications & $11.9 \%$ & $8.3 \%$ & 0.4 \\
Renal replacement therapy & $20.8 \%$ & $13.7 \%$ & 0.2 \\
\hline
\end{tabular}

$\mathrm{ICU}=$ Intensive care unit.

* CABG-Coronary artery bypass grafting.

** Data expressed as median (Range).

*** Data expressed as mean (SD).

collected prospectively between 2005 and $2012(\mathrm{~N}=256)$. Patients were categorised as: Complex valve surgery (multiple valve surgery, valve with CABG and aortic dissection with valve surgery) $\mathrm{N}=182$ and isolated valve surgery $\mathrm{N}=74$. Quality of life questionnaire (SF12) was only sent to patients who were alive and at least 1 year post surgery $(\mathrm{N}=162)$. Results: There was female preponderance in isolated valve group $(64 \% \mathrm{v} / \mathrm{s} 37 \%, \mathrm{p}=0.01)$. There was no significant difference in the incidence of diabetes, peripheral vascular disease, lung disease and the urgency of surgery between the two groups. Octogenarians undergoing isolated valve surgery tended to have preserved LV function ( $84 \% \mathrm{vs.} 62 \%$, $\mathrm{p}<0.01)$. There was no significant difference between the two groups in terms of re-exploration, ICU readmission, renal replacement therapy, chest infection, cerebrovascular and gastrointestinal complications. The complex surgery group required more airway support post-op ( $90 \%$ vs. $80 \%, \mathrm{p}=0.02)$ and showed higher infection (wound and urine) rates (30.5\% vs. $15.5 \%, \mathrm{p}=$ 0.02 ). Although there was a statistically significant difference in 30 -day mortality, there was no such difference in late mortality in the groups at a mean follow-up of 40 months. $92 \%$ of the patients returned the SF12. This showed no significant difference in the physical and mental test scores between the groups $(\mathrm{p}=0.3)$. Conclusion: Long term outcomes and quality of life in octogenarians after complex valve surgery is comparable to patients undergoing isolated valve surgery. This data also showed lower mortality and better post-operative morbidity than reported in the Partner Trial.

\section{Left Atrial Mid Cross Sectional Area and Left Atrial Shape Index: Novel Echocardiographic Measures Which Mechanistically Reflect Risk of Atrial Fibrillation and Stroke}

\author{
Timothy Tan, Maria C. Nunes, Mark Handschumacher, Judy Hung \\ Massachusetts General Hospital, Boston, MA, USA
}

Objective: Left atrial (LA) remodelling is a significant risk factor for atrial fibrillation (AF) and stroke particularly in valvular disease such as rheumatic mitral stenosis (MS). Typically, LA remodelling results in increase in size and change in morphology of the LA. Our aim was to develop novel and simple echocardiographic measures for LA size and shape reflective of LA flow dynamics that can be applied in a clinical setting to improve risk stratification of $\mathrm{AF}$ and stroke. Methods: Conventional and novel 2D echo measures of LA size were examined in a prospective cohort of 1275 ischemic stroke patients (IS) in the context of etiology of stroke and AF. Specifically, mid LA cross sectional area (LA CSA) which reflects both LA size and shape, was calculated using the formula $\pi / 4 \mathrm{x}$ largest measured LA diameter $\mathrm{x}$ smallest measured LA diameter at end-systole where mid LA diameter was measured in the parasternal long axis, 4 chamber and 2 chamber views. LA shape and its association with embolic stroke was then examined in a separate cohort of 212 patients with rheumatic MS where LA shape was expressed as ratio of measured LA end-systolic volume to hypothetical sphere volume $\left(4 / 3 \pi r^{3}\right.$ where $r=$ radius obtained from 3D LA CSA $)$. Results: In the cohort of IS patients, increased LA CSA (8.6 \pm 2.3 vs. $6.4 \pm 1.8$ $\left.\mathrm{cm}^{2} / \mathrm{m}^{2} ; \mathrm{p}<0.001\right)$ was associated with cardioembolic strokes. Patients with AF also had increased LA CSA $\left(8.7 \pm 2.5 \mathrm{vs} .6 .3 \pm 1.7 \mathrm{~cm}^{2} /\right.$ $\left.\mathrm{m}^{2} ; \mathrm{p}<0.001\right)$ compared to sinus rhythm which was significant after adjustment for the effects of age, gender, hypertension, E/E' and LVEF (OR 1.6; 95\% CI 1.46 to 1.74 ; $\mathrm{p}<0.001$ ). Of the 212 patients with MS, 41 had a stroke. In multivariate analysis, LA 3D emptying fraction (adjusted OR 0.95; 95\% CI 0.91 to $0.98 ; \mathrm{p}=0.012$ ) and LA shape index (OR $0.69 ; 95 \%$ CI 0.57 to $0.85 ; \mathrm{p}<0.001$ ) provided incremental predictive value for stroke beyond age and AF. Conclusions: LA CSA and LA shape index are novel echocardiographic measures which mechanistically reflect risk of AF and stroke, and may potentially be useful in the clinical setting for risk stratification of AF and stroke.

\section{Postoperative Neurohormonal Activation in Patients with Organic Mitral Regurgitation: Clinical Profile and Prognostic Implications}

\section{Rodolfo Pizarro, Pablo F. Oberti, Mariano L. Falconi, Alberto Domenech, Anibal M. Arias, Juan G. Krauss, Ricardo G. Marenchino, Vadim Kotowicz, \\ Jose Luis C. Navarro Estrada, Arturo M. Cagide, Cesar A. Belziti \\ Hospital Italiano de Buenos Aires, Buenos Aires, Argentina}

Background: Neurohormonal activation is present in organic valve disease and its assessment is of prognostic value. However, it has not been fully assessed during the postoperative (PO) pe- 
riod of patients (pts) with organic mitral regurgitation (MR). Objective: To establish the prognostic value of brain natriuretic peptide (BNP) in the PO period of pts undergoing valve surgery due to organic MR. Methods: A total of 217 pts (mean age $63 \pm 8$ years; $68 \%$ male) with organic MR and an ejection fraction (EF) of $62 \pm 6 \%$ undergoing mitral valve surgery were assessed prospectively and consecutively; 103 pts had valve repair and 114 pts had valve replacement. The combined end point was death and/or hospitalization due to heart failure. Mean follow up was $4.5 \pm 2.3$ years. Results: During the PO period, mean BNP values at 24 hours, 7 days, 1 month and 3 months were $131 \pm 77 \mathrm{pg} / \mathrm{ml}, 123 \pm 65$ $\mathrm{pg} / \mathrm{ml}, 106 \pm 43 \mathrm{pg} / \mathrm{ml}$ and $93 \pm 34 \mathrm{pg} / \mathrm{ml}$, respectively. Nine $\mathrm{p}$ died (4\%) and $26 \mathrm{p}$ (11.9\%) were hospitalized due to heart failure during follow up. The mean BNP value in $\mathrm{p}$ with events vs. no-events was $109 \pm 19 \mathrm{pg} / \mathrm{ml}$ vs. $54 \pm 13 \mathrm{pg} / \mathrm{ml}, \mathrm{p}<0.001$. After a 6 month follow up, BNP levels $\geq 100 \mathrm{pg} / \mathrm{ml}$ were associated to the end point; OR: 4.56 (95\% CI: 2.93-17.82, $\mathrm{p}<0.001)$. An elevated BNP level correlated with PO EF $(\mathrm{R} 2=0.57, \mathrm{p}<0.0001)$, the presence of atrial fibrillation (AF) in pts who were in sinus rhythm prior to surgery $(\mathrm{R} 2=0.52, \mathrm{p} 1+(\mathrm{R} 2=0.43, \mathrm{p}<0.002))$. After adjusting for clinical, echocardiographic and surgical variables, BNP level (RR: 3.53 (2.45-19.5, p < 0.001)), PO EF <55\% (RR: 3.47 (2.717.4, $\mathrm{p}<0.01)$ ) and persistent AF (RR: $3.12(2.32-21.3, \mathrm{p}<0.02))$ were associated to the end point. The ROC area of the model including BNP had better discriminatory ability compared to that of the model not including BNP (PO EF $1+)$, (0.84 vs. 0.75 , p < $0.001)$. Conclusion: Persistently high values after the 6th PO month $(\geq 100 \mathrm{pg} / \mathrm{ml})$ are significantly associated to events. Hence, BNP levels during follow up allows to define subgroups of pts at higher risk.

\section{Right Ventricular Outflow Tract Replacement in Ross Procedures Is the Performance of Bioprothesis Equivalent to Homografts?}

\section{Aleksandra Miskovic, Nadejna Monsefi, Afsaneh Karimian, Anton Moritz \\ Department of Thoracic- and Cardiovascular Surgery, Johann Wolfgang-Goethe University Hospital, Fran, Frankfurt am Main, Germany}

Background: Homograft is the gold standard for reconstruction of the right ventricular outflow tract in patients undergoing the Ross procedure for aortic valve replacement. But costs and lack of availability increased the need for alternatives. We evaluated outcome of homograft vs. bioprosthetic RVOT replacement after the Ross procedure in adults. Methods: Between 1996 and 2013 a total of 205 adult patients ( 157 male, mean age $43 \pm 10$ ) underwent aortic root replacement with a pulmonary autograft. The RVOT was replaced with a homograft in 113 patients and with a stentless bioprosthesis (Freestyle, Medtronic) in 92. In 37 Patients with bioprostehsis an additional surgical procedure by interponing a pericard sleeve was performed to avoid stricture at the proximal suture line. Patients were followed for a mean of 8 years (range 1 to 17, 1556 patient years). Results: A total of 17 Patient died during follow up $(8.3 \%)$. Mean survival time did not differ between the groups (p log rank: 0.5) 23 patients required reintervention, mostly because of developing pulmonary stenosis $(n=20)$, caused by degeneration or endocarditis (mostly in homograft) or early stricture at the proximal suture line (mostly in Bioprosthesis). Significant difference was found for the time and incidence rate between the groups In patients with homograft the incidence rate for Reintervention was 1 in 139 patient years, mean time interval $9( \pm 5)$ years. In the bioprothesis group the incidence rate was 1 in 35 pat. years after a mean time of $4( \pm 3)$ years. Incidence rate ratio difference was about 5 between the groups (CI 4, 3-5.2, $\mathrm{p}<0.0001)$. The main problem with proximal stricture in the Bioprosthesis group could be successfully treated by interponing a pericard sleeve at the suture line, reducing the reintervention rate from 20 to $5 \%$ ( $\mathrm{p}=$ 0.04). Conclusions: Patients with bioprotheses in RVOT position after the Ross procedure showed a significantly higher risk of reintervention or pulmonary valve dysfunction. The main problem, early development of a stricture at the proximal suture line can be solved by interponing a pericard sleeve to achieve satisfactory bioprosthetic function in the RVOT.

\section{Aortic Stenosis with Low Gradient and Normal Ejection Fraction: Clinical Value of Natriuretic Peptides}

Rodolfo Pizarro, Pablo F. Oberti, Mariano L. Falconi, Anibal M. Arias, Juan G. Krauss, Arturo M. Cagide, Cesar A. Belziti

Hospital Italiano de Buenos Aires, Buenos Aires, Argentina

Background: The clinical significance of aortic stenosis (AS) with a low gradient, valve area $<1 \mathrm{~cm}^{2}$ and normal ejection fraction (EF) remains controverted. The relevance of natriuretic peptides (NP) in this disorder has not been assessed until present. Objective: To assess the clinical significance of NP in patients (pts) with AS and low gradient, decreased valve area and normal EF. Methods: A total of 106 pts with AS were prospectively included (mean age $74 \pm 11 \mathrm{y}, 57 \%$ male, history of hypertension in $67 \%$, aortic valve area $<1 \mathrm{~cm}^{2}$, mean gradient $55 \%$ ). The value of NT-pro BNP associated to clinical and echocardiographic variables was assessed. Study end points were cardiac death and/or progression of dyspnea. Mean follow up was $2.6 \pm 1.3$ years. Results: Of the $106 \mathrm{pts}, 6 \mathrm{p}$ died due to cardiac causes (5.6\%) and 39 $\mathrm{p}(36 \%)$ suffered progression of dyspnea. Mean NT-pro BNP value was $1295 \pm 458$. Patients with events had higher mean NT-pro BNP values than pts without events $(1977 \pm 645$ vs. $876 \pm 401$, p $1100 \mathrm{pg} / \mathrm{ml}$; sensitivity and specificity values of the ROC curve for end point detection were $81 \%$ and $87 \%$, respectively. Adjusting for clinical and echocardiographic variables, NT-pro BNP $>1100$ pg/ml (RR: 4.15, 95\% CI: 2.32-25.4, p75 years had better event discrimination ability (ROC area 0.89 vs. $0.79, \mathrm{p}<0.0001$ ). Conclusion: In pts with $\mathrm{AS}$, a mean gradient $<40 \mathrm{~mm} \mathrm{Hg}$, aortic valve area $<1 \mathrm{~cm}^{2}$ and normal EF, NP's could differentiate a population at greater risk, independently from the degree of valve calcification and LV mass. 


\section{A Method of Preparing a Living Autologous Pericardial Tissue Heart Valve Replacement That Is Modified and Stabilized Using in Vitro Three-Dimensional Mechanotransduction}

\author{
Frantisek Straka ${ }^{1}$, David Schornik ${ }^{2}$, Jaroslav Masin ${ }^{1}$, Elena Filova ${ }^{2}$, \\ Zuzana Burdikova ${ }^{3}$, Tomas Mirejovsky ${ }^{1}$, Hynek Chlup ${ }^{4}$, \\ Lukas Horny ${ }^{4}$, Jan Vesely ${ }^{4}$, Jan Pirk', Lucie Bacakova ${ }^{2}$ \\ ${ }^{1}$ Institute for Clinical and Experimental Medicine, Prague, \\ Czech Republic; ${ }^{2}$ Dept. of Biomaterials and Tissue Engineering, \\ Institute of Physiology Academy of Sciences, Prague, \\ Czech Republic; ${ }^{3}$ Department of Biomathematics, Institute \\ of Physiology, Prague, Czech Republic; ${ }^{4}$ Laboratory of \\ Biomechanics, Faculty of Mechanical Engineering, Czech \\ Technical University, Prague, Czech Republic
}

Background: The objective of our study was to prepare an autologous living pericardial heart valve replacement. In vitro dynamic conditioning was used to modify the pericardial tissue through the process of three-dimensional (3D) mechanotranduction. Methods: Living human pericardium was harvested during heart surgery and a stented heart valve replacement was made from the pericardium. The heart valve replacement was then subjected to the same systemic pressures during repetitive heart contraction-relaxation cycles simulated in a bioreactor using a pulsatile pump. Native aortic heart valves were harvested during heart transplantation and were compared with the autologous human pericardium before and after dynamic conditioning using histological assessment, immunohistochemical analysis and confocal microscopy. The biomechanical properties of both tissues were also compared. Results: In vitro dynamic conditioning using an anatomical 3D stent stimulates the living pericardial intersticial cells (PICs) within the autologous pericardium to proliferate and differentiate to an active phenotype (aPICs) which produces and remodels the pericardial extracellular matrix (ECM) giving the pericardial tissue the property of anisotropy similar to that of the normal aortic heart valve. Collagen, elastin and glycosaminogly-

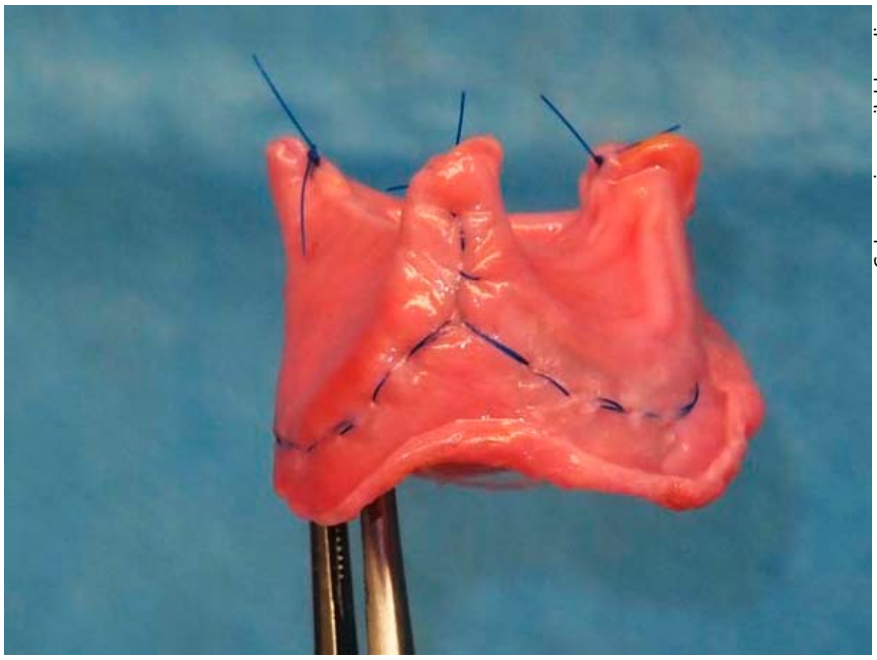

Fig. 1. (for Abstract of Frantisek Straka). can production by PICs is substantially increased ( $\mathrm{p} \leq 0.001)$, modifying the pericardial tissue to resemble the tissue of a normal human heart valve. This tissue is stronger, yet soft and pliable, possessing biomechanical properties similar to normal aortic heart valve cusp tissue based on secant elastic modulus (18-27 MPa). Hemodynamic properties measured by echocardiography such as transvalvular Doppler gradient $(5-6 \mathrm{~mm} \mathrm{Hg}$ ) and valve cusp coaptation were also comparable to the native aortic heart valve. Conclusions: Human living autologous pericardium is a favorable tissue for preparing a heart valve replacement for potential clinical use. This tissue resembles the natural structure of the normal aortic heart valve, and has similar mechanical and hemodynamic properties. PICs are activated to an active myofibroblast-like phenotype through mechanical conditioning allowing the production and remodeling of the pericardial ECM. Acknowledgements: Supported by the Grant Agency of the MH of the CR (No. NT 11270).

\section{Mid-Term Follow-Up of Haemodynamic Performance of the St. Jude Medical Trifecta Aortic Bioprosthesis in Young Patients Under 65}

\author{
Amir Sepehripour, Jacob Chacko, Kulvinder Lall
}

St Bartholomew's Hospital, London, United Kingdom

Objective: The St. Jude Medical Trifecta aortic supra-annular bioprosthesis is regarded as the next generation in pericardial stented tissue valves. The unique design of tissue leaflets attached to the exterior of the valve stent provides unrivalled in-vivo mean gradients and haemodynamics. The aim of this prospective study was to evaluate midterm haemodynamic performance of valve implanted into patients under 65. Methods: Twenty three consecutive patients undergoing aortic valve replacement using the St. Jude Medical Trifecta valve at a single UK centre over a 36-month period were included in this study. Patients undergoing concomitant cardiac procedures were included. All implanted valves were 19, 21, 23, 25 and $27 \mathrm{~mm}$ in size. Assessment of haemodynamic function was carried out using transthoracic echocardiography pre-operatively and at follow-up, as well as transoesophageal echocardiography intra-operatively. Results: The study population consisted of 23 patients (12 male, 11 female). Mean age was $60.7 \pm 2.1$ years. Implanted valve sizes were $19 \mathrm{~mm}$ $(\mathrm{n}=3), 21 \mathrm{~mm}(\mathrm{n}=6), 23 \mathrm{~mm}(\mathrm{n}=7), 25 \mathrm{~mm}(\mathrm{n}=4)$ and $27 \mathrm{~mm}$ $(\mathrm{n}=3)$. Overall mean post-operative pressure gradients were $7.34 \pm 4.2 \mathrm{~mm} \mathrm{Hg}$ (mean) and $14.31 \pm 4.8 \mathrm{~mm} \mathrm{Hg}$ (peak). Subgroup mean post-operative pressure gradients were $7.35 \pm 2.8 \mathrm{~mm} \mathrm{Hg}$, $7.62 \pm 2.1 \mathrm{~mm} \mathrm{Hg}, 7.94 \pm 3.7 \mathrm{~mm} \mathrm{Hg}, 7.61 \pm 2.7 \mathrm{~mm} \mathrm{Hg}$, $7.21 \pm 2.8 \mathrm{~mm} \mathrm{Hg}$, for the 19, 21, 23, 25 and $27 \mathrm{~mm}$ cohort respectively. Overall mean post-operative left ventricular ejection fraction was $62 \pm 1.7 \%$. Overall mean effective orifice area was $1.86 \pm 0.4 \mathrm{~cm}^{2}$. There were only 2 patients with trivial regurgitation. Conclusions: These results of our experience demonstrate excellent haemodynamic performance of the Trifecta bioprosthetic valve in young patients under 65 . 


\section{Antegrade Cardioplegia for Surgery of the Aortic Root Appears Unnecessary}

Dustin Hang ${ }^{1}$, Alan Markowitz ${ }^{2}$

${ }^{1}$ Case Western Reserve University School of Medicine, Cleveland, $\mathrm{OH}, \mathrm{USA}^{2}{ }^{2}$ University Hospitals, Cleveland, $\mathrm{OH}, \mathrm{USA}$

Background: Administration of antegrade cardioplegia (ACP) is cumbersome in the open aortic root. In a series of almost 2,000 aortic valve procedures since 2000 , retrograde cardioplegia (RCP) exclusively was used in the setting of severe aortic regurgitation or severely diseased ascending aortas with no effort made to cannulate the coronary ostia. Methods: Retrospective study of 271 patients undergoing stentless aortic valve replacement or root replacement \pm ascending aortic/arch; 70 were excluded due to patent mammary grafts or addition of SVG's through which ACP was administered. Of 201 patients with RCP only, 27\% were redo's, AVR-74; Root replacement-44; Root and ascending aorta/hemi or full arch-83. Crossclamp (XCL) times were divided into three groups: $\leq 90$ (XCI-1); 91150(XCI-2); >150(XCI-3). Chi-square analysis was used for univariate analysis of categorical variables and ANOVA for continuous variables. Results: Overall mortality was 3.5\%; mortality was 3.64\% for redo's and $3.42 \%$ for virgin operations $(p=0.94) .1$ LVAD was placed for profound LV dysfunction due to unrecognized cold agglutinins; and 5 IAB's were employed (3 pre-op). Conclusions: The success of myocardial preservation during open aortic procedures, as reflected by survival and complication rates, with RCP alone compares favorably with ACP only or ACP/RCP combination. In the literature, mortality ranges from $6.7-13.3 \%$ for aortic root replacement, $4.8 \%$ for aortic root+ascending aorta; mortality for reoperative aortic root replacement after previous aortic root procedures was reported at $11.5 \%$, and $12 \%$ for reoperations on the aortic root/ ascending aorta for aneurysm, false aneurysm, or infectious disease. The extra effort required to administer ACP appears unnecessary.

Table 1. Continuous variables as mean \pm st. dev., $\mathrm{XCL}=$ cross clamp, $\mathrm{CPB}=$ cardiopulmonary bypass, $\mathrm{MORT}=$ mortality, $\mathrm{CVA}=$ cerebrovascular accident (for Abstract of Dustin Hang)

\begin{tabular}{lllll}
\hline & XCI-1(34) & XCI-2(110) & XCI-3(57) & p value \\
\hline XCL TIME (min) & $74 \pm 9$ & $123 \pm 16$ & $182 \pm 30$ & 0.00 \\
CPB (min) & $112 \pm 28$ & $163 \pm 30$ & $236 \pm 48$ & 0.00 \\
MORT & $2.9 \%$ & $3.6 \%$ & $3.5 \%$ & 0.98 \\
CVA & $2.9 \%$ & $1.8 \%$ & $1.7 \%$ & 0.91 \\
\hline
\end{tabular}

\section{Mid-Term Follow-Up Of Haemodynamic Performance of the St. Jude Medical Trifecta Aortic Bioprosthesis in Octogenarians}

\section{Amir Sepehripour, Jacob Chacko, Kulvinder Lall \\ St Bartholomew's Hospital, London, United Kingdom}

Objective: The St. Jude Medical Trifecta aortic supra-annular bioprosthesis is regarded as the next generation in pericardial stented tissue valves. The unique design of tissue leaflets attached to the exterior of the valve stent provides unrivalled in-vivo mean gradients and haemodynamics. The aim of this prospective study was to evaluate midterm haemodynamic performance of valve implanted into patients over 80 . Methods: Thirty nine consecutive patients undergoing aortic valve replacement using the St. Jude Medical Trifecta valve at a single UK centre over a 36-month period were included in this study. Patients undergoing concomitant cardiac procedures were included. All implanted valves were 19, 21, 23, 25 and $27 \mathrm{~mm}$ in size. Assessment of haemodynamic function was carried out using transthoracic echocardiography pre-operatively and at follow-up, as well as transoesophageal echocardiography intra-operatively. Results: The study population consisted of 39 patients ( 23 male, 16 female). Mean age was $82.3 \pm 1.2$ years. Implanted valve sizes were $19 \mathrm{~mm}(\mathrm{n}=$ $7), 21 \mathrm{~mm}(\mathrm{n}=12), 23 \mathrm{~mm}(\mathrm{n}=13), 25 \mathrm{~mm}(\mathrm{n}=5)$ and $27 \mathrm{~mm}$ $(\mathrm{n}=2)$. Overall mean post-operative pressure gradients were $8.12 \pm 3.5 \mathrm{~mm} \mathrm{Hg}$ (mean) and $14.27 \pm 5.7 \mathrm{~mm} \mathrm{Hg}$ (peak). Subgroup mean post-operative pressure gradients were $8.23 \pm 5.1 \mathrm{~mm} \mathrm{Hg}$, $7.83 \pm 1.5 \mathrm{~mm} \mathrm{Hg}, 8.15 \pm 2.6 \mathrm{~mm} \mathrm{Hg}, 7.38 \pm 2.4 \mathrm{~mm} \mathrm{Hg}$, $7.14 \pm 2.3 \mathrm{~mm} \mathrm{Hg}$, for the 19, 21, 23, 25 and $27 \mathrm{~mm}$ cohort respectively. Overall mean post-operative left ventricular ejection fraction was $57 \pm 2.4 \%$. Overall mean effective orifice area was $1.73 \pm 0.9 \mathrm{~cm}^{2}$. There were only 4 patients with trivial regurgitation. Conclusions: These results of our experience demonstrate excellent haemodynamic performance of the Trifecta bioprosthetic valve in octogenarians.

\section{Mid-Term Follow-Up of Haemodynamic Performance of the St. Jude Medical Trifecta Aortic Bioprosthesis}

Amir Sepehripour, Jacob Chacko, Kulvinder Lall

St Bartholomew's Hospital, London, United Kingdom

Objective: The St. Jude Medical Trifecta aortic supra-annular bioprosthesis is regarded as the next generation in pericardial stented tissue valves. The unique design of tissue leaflets attached to the exterior of the valve stent provides unrivalled in-vivo mean gradients and haemodynamics. The aim of this prospective study was to evaluate midterm haemodynamic performance. Methods: One hundred and thirty five consecutive patients undergoing aortic valve replacement using the St. Jude Medical Trifecta valve at a single UK centre over a 36-month period were included in this study. Patients undergoing concomitant cardiac procedures were included. All implanted valves were 19, 21, 23, 25 and $27 \mathrm{~mm}$ in size. Assessment of haemodynamic function was carried out using transthoracic echocardiography pre-operatively and at follow-up, as well as transoesophageal echocardiography intra-operatively. Results: The study population consisted of 135 patients (73 male, 62 female). Mean age was $77.5 \pm 5.2$ years. Implanted valve sizes were $19 \mathrm{~mm}(\mathrm{n}=13), 21 \mathrm{~mm}(\mathrm{n}=35), 23 \mathrm{~mm}$ $(\mathrm{n}=57), 25 \mathrm{~mm}(\mathrm{n}=23)$ and $27 \mathrm{~mm}(\mathrm{n}=7)$. Overall mean postoperative pressure gradients were $7.46 \pm 3.7 \mathrm{~mm} \mathrm{Hg}$ (mean) and $15.36 \pm 4.9 \mathrm{~mm} \mathrm{Hg}$ (peak). Subgroup mean post-operative pressure gradients were $7.26 \pm 7.2 \mathrm{~mm} \mathrm{Hg}, 7.56 \pm 2.9 \mathrm{~mm} \mathrm{Hg}$, $8.67 \pm 4.3 \mathrm{~mm} \mathrm{Hg}, 7.41 \pm 3.9 \mathrm{~mm} \mathrm{Hg}, 7.36 \pm 2.9 \mathrm{~mm} \mathrm{Hg}$, for the 19 , 
21, 23, 25 and $27 \mathrm{~mm}$ cohort respectively. Overall mean postoperative left ventricular ejection fraction was $61 \pm 3.1 \%$. Overall mean effective orifice area was $1.81 \pm 0.5 \mathrm{~cm}^{2}$. Moderate-severe paravalvular leak was observed in one patient and mild-moderate leak in another. Otherwise there were only 17 patients with trivial regurgitation. Conclusions: These results of our experience demonstrate excellent haemodynamic performance of the Trifecta bioprosthetic valve.

\section{Mid-Term Follow-Up of Haemodynamic Performance of the St. Jude Medical Trifecta Aortic Bioprosthesis - The Training Perspective}

\section{Amir Sepehripour, Jacob Chacko, Kulvinder Lall}

St Bartholomew's Hospital, London, United Kingdom

Objective: The Trifecta aortic bioprosthesis provides exceptional haemodynamic performance, durability and implantability. The unique design, encompassing a contoured silicone insert within a unique cuff is specifically designed to conform to the native annulus shape for proper seating and minimal risk of paravalvular leak. The aim of this prospective study was to evaluate haemodynamic performance of valves implanted by cardiothoracic trainees at a single UK centre. Methods: Twenty-one consecutive patients undergoing aortic valve replacement using the Trifecta valve performed by trainees at a single UK centre over a 36-month period were included. Patients undergoing concomitant cardiac procedures were included. Assessment of haemodynamic function was carried out using transthoracic echocardiography pre-operatively and at follow-up, as well as transoesophageal echocardiography intra-operatively. Results: The study population consisted of 21 patients ( 14 male, 7 female). Mean age was $72.4 \pm 7.6$ years. Implanted valve sizes were $19 \mathrm{~mm}(\mathrm{n}=1), 21 \mathrm{~mm}(\mathrm{n}=7), 23 \mathrm{~mm}$ $(\mathrm{n}=9), 25 \mathrm{~mm}(\mathrm{n}=3)$ and $29 \mathrm{~mm}(\mathrm{n}=1)$. Overall mean postoperative pressure gradients were $6.47 \pm 1.7 \mathrm{~mm} \mathrm{Hg}$ (mean) and $13.42 \pm 5.3 \mathrm{~mm} \mathrm{Hg}$ (peak). Subgroup mean post-operative pressure gradients were $7.21 \pm 3.1 \mathrm{~mm} \mathrm{Hg}, 7.51 \pm 3.1 \mathrm{~mm} \mathrm{Hg}, 7.63 \pm 2.4 \mathrm{~mm} \mathrm{Hg}$, $7.31 \pm 4.6 \mathrm{~mm} \mathrm{Hg}, 7.46 \pm 4.9 \mathrm{~mm} \mathrm{Hg}$, for the 19, 21, 23, 25 and $27 \mathrm{~mm}$ cohort respectively. Overall mean post-operative left ventricular ejection fraction was $56 \pm 0.12 \%$. Overall mean effective orifice area was $1.63 \pm 0.7 \mathrm{~cm}^{2}$. All valves were well-seated and only 2 exhibited trivial regurgitation. Conclusions: Our experience demonstrates the excellent haemodynamic performance of the Trifecta bioprosthesis in implantations performed by surgical trainees, resulting from the innate superior haemodynamic properties of the Trifecta valve as well as the simple yet faultless implantability of the valve.

\section{Learning Curve: Comparison of Early Experience in Cohort B During Partner Trial to Recent Results in Post Approval Era}

\author{
Elizabeth K. Walsh, Nimesh Desai, Wilson Szeto, \\ Prashanth Vallabhajosyula, Saif Anwaruddin, Jay Giri, \\ Howard Herrmann, Joseph Bavaria
}

University of Pennsylvania, Philadelphia, PA, USA

Objective: By analyzing your Transcatheter Aortic Valve (TAVR) program a site will understand the impact of patient selection, procedure techniques and post-operative care of the TAVR patient. The purpose of the study was to understand effect of the learning curve on our TAVR program. Methods: We looked at 42 patients from Partner 1 cohort B (P1B) implanted 11/2007-7/2009, 102 patients from Partner llB (PllB) implanted 7/2011-11/2013 and 186 patients with commercial Sapien implanted 2/201210/2013. A comparison of patient's age, Society of Thoracic Surgery risk score (STS) and gender was prepared. A Kaplan-Meier (KM) survival curve was performed. Results: There was no significant difference of ages in all 3 cohorts with $\mathrm{P}$ value $=0.562 \%$. There was a significantly higher incidence of female patients in the commercial cohort with a $\mathrm{P}$ value $0.002 \%$. The P1B cohort had a significantly higher occurrence in the commercial population with a $\mathrm{p}$ valve $=0.29 \%$. The $\mathrm{KM}$ demonstrated a significantly higher mortality in P1B cohort compared to P2B or commercial (fig. 1). Conclusions: Programs will experience a learning curve. Shortening the learning curve requires continued education on patient selection, technical experience and care of the TAVR patient. As the technology continues to evolve and devices improve so will the demand for TAVR. It is crucial that educational needs are met as your Heart Team grows.

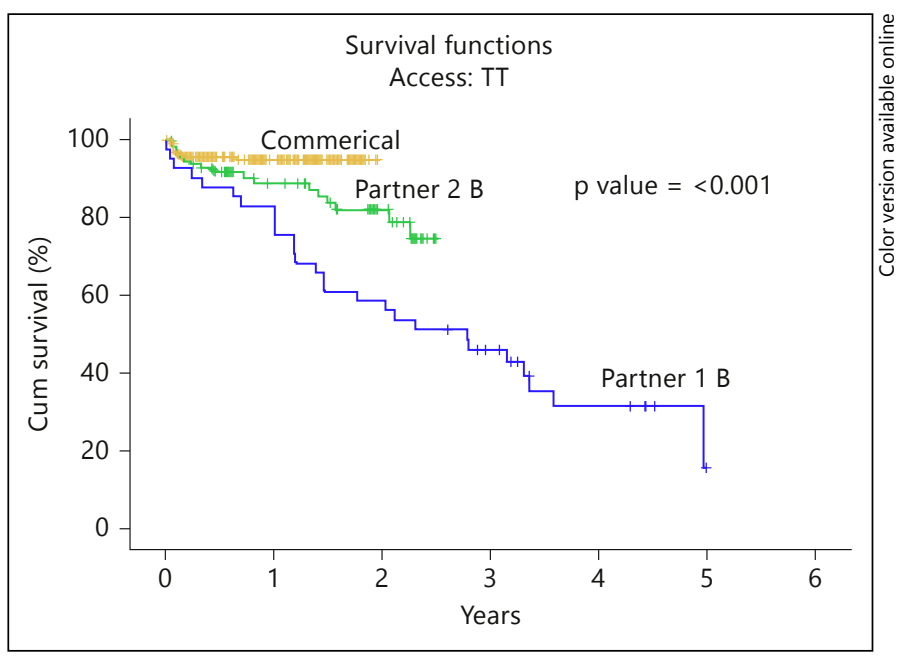

Fig. 1. (for Abstract of Elizabeth K. Walsh). 


\section{Long-Term Clinical Results with the '3F' Aortic Bioprosthesis}

\section{Joerg Linneweber, Benjamin Claus, Wolfgang Konertz}

Charité Universitätsmedizin Berlin, Berlin, Germany

Objective: The $3 \mathrm{~F}$ aortic bioprothesis is an equine pericardial stentless valve for aortic valve replacement. This study reports the long-term clinical outcome and hemodynamic performance of the prosthesis. Methods: 75 patients received the prosthesis between 2002 and 2007 at our institution. Clinical outcome was assessed by telephone interviews with the primary care physicians $(n=40)$. Long-term hemodynamic performance of the valve was evaluated by transthoracic echocardiography $(\mathrm{n}=35)$ at our institution. Results: Mean follow-up was 92 (72 to 134) months and 64\% complete. Overall survival rate was $77 \%$. Freedom from reoperation was $97 \%$. Two patients required reoperation due to prosthetic valve endocarditis. SAE included permanent and transient neuroembolic events. However, non of the events seemed to be associated with the device; the incidence of at least paroxysmal atrial fibrillation was $31 \%$. $75 \%$ of the ten-years survivors (mean age 80.1[[Unsupported Character - Symbol Font \&\#61617;]]5.4; range 71-88 years) received no oral anticoagulation. A total of 166 valve years have been evaluated by TTE (116 to 134 months follow-up). No severe structural or nonstructural valve dysfunction was revealed; mean transvalvular pressure gradient (Pmean) was $14.3 \pm 12.8 \mathrm{~mm} \mathrm{Hg}$ and showed no significant increase compared to postoperative values. Accordingly, postoperative NYHA class improvement showed good results in this elderly patient cohort. Conclusions: The $3 \mathrm{~F}$ aortic valve prosthesis demonstrated excellent durability and good long-term clinical results.

\section{The Transapical Neochord Approach for Mitral Regurgitation in Four Selected Cases}

\section{Andrea Colli', Fabio Zucchetta' ${ }^{1}$ Erica Manzan', Giorgio Pittarello', Cristiano Sarais', Gino Gerosa', Giovanni Speziali $^{2}$}

${ }^{1}$ University of Padova, Padova, Italy; ${ }^{2}$ University of Pittsburgh, Pittsburgh, PA, USA

Objective: The off-pump transapical implantation of artificial chordae tendinae is a recent mitral valve repair technique that enhances the 'respect rather than resect' concept in mitral valve surgery through a real minimally invasive procedure. We retrospec- tively analyzed 4 surgical high-risk cases of mitral valve regurgitation (MR) with different clinical and anatomical characteristics referred to NeoChord implantation. Methods: We selected 3 female and 1 male patients with severe symptomatic MR (New York Heart Association - NYHA functional class IV) with a median age of 82 years. They had an high risk profile for traditional surgery because of multiple comorbidities such as previous percutaneous coronary intervention for coronary artery disease, chronic obstructive pulmonary disease, post-actinic pericarditis, chronic renal failure, bladder and bowel bleeding polyps. One patient presented an anterior leaflet prolapse, meanwhile the others had posterior leaflet prolapse; two patients showed left ventricular dilation and dysfunction with severe pulmonary hypertension, one with extended annular calcification too. They underwent multiple offpump GoreTex neochords implantation on the prolapsed leaflet under real time 2D/3D transesophageal echocardiographic monitoring. Results: Neither surgical nor clinical complications were recorded. Two patients were discharged without postoperative MR; two patients showed mild residual MR due to the complex anatomical substrate. A marked improvement of clinical conditions with regression to NYHA class I was achieved in all cases besides the residual MR. All patients were in sinus rhythm needing only antiplatelet therapy. Conclusions: the Neochord procedure proved to be safe and effective in the 4 treated patients. These satisfactory results raise the prospect of extending this technique in younger patients with lower surgical risk before ventricular remodeling occurs. Further studies are needed to demonstrate NeoChord long term durability.

\section{Mini-Thoracotomy for Mitral Valve Repair: Cosmetics or Less Invasive?}

Marco Diena Bogdan Adrian Popa, Gheorghe Cerin, Eugenio Novelli, Angelo Romano, Levan Karazanishvili, Gabriele Musica

San Gaudenzio Clinic, Novara, Italy

Objective: Mini-thoracotomy carries a cosmetic advantage compared to sternotomy but is not clearly proved its clinical benefit in term of a real less invasiveness. This analysis compared two groups of patients affected by degenerative mitral regurgitation (MR) in which the surgical repair was performed through median sternotomy (Group 1) or right mini-thoracotomy (Group 2). Methods: Data from 555 patients who underwent MV surgery from January'09 to December' 13 were prospectively collected and analysed. For patient's characteristics see table 1 . Within the min-

Table 1. Patients' characteristics and results (for Abstract of Marco Diena)

\begin{tabular}{llllllllllll}
\hline Group & Nr. & Male (\%) & Age & LVEF & EScore & $\begin{array}{l}\text { EC } \\
\text { time }\end{array}$ & $\begin{array}{l}\text { ACC } \\
\text { time }\end{array}$ & $\begin{array}{l}\text { Periop } \\
\text { bleeding }(\mathrm{ml})\end{array}$ & $\begin{array}{l}\text { ICU } \\
\text { stay (d) }\end{array}$ & $\begin{array}{l}\text { Transfusion } \\
(\%)\end{array}$ & Mech. ventilat. (h) \\
\hline $1(\mathrm{~S})$ & 373 & $196(52.7)$ & $65.7 \pm 14$ & $56.5 \pm 10$ & 5.9 & $97^{\prime}$ & $65^{\prime}$ & 520 & 3.1 & $196(52.5)$ & 22.8 \\
$2(\mathrm{M})$ & 182 & $126(69.1)$ & $55.7 \pm 12$ & $61.6 \pm 6$ & 3.1 & $110^{\prime}$ & $77^{\prime}$ & 280 & 2.3 & $34(18.7)$ & 19.4 \\
$\mathrm{p}$ & - & & - & - & - & na & na & $<0.001$ & 0.652 & $<0.001$ & na \\
\hline
\end{tabular}


imally invasive approach the cardiopulmonarybypass was achieved through a percutaneous venous cannula in the groin and a direct ascending aorta cannulation. A flexible aortic cross clamp was applied through the skin incision and cardioplegic arrest was obtained with delivery of antegrade Custodiol ${ }^{\circledR}$ HTK solution. Surgical strategy aimed to correct the MR with a single orifice in both groups according to Carpentier rules except for the treatment of AML prolapse in which we used PTFE neochordae sutures. PML prolapse was corrected by either quadrangular or triangular resection. Statistical analysis: in order to control the effect of possible confounding variables, we have used the model of multivariate regression with age, gender, LVEF and the surgical approach as regressors. Results: The main results are listed in table 1. Follow-up was completed in $95 \%$ of patients. At mean 2.2 years of follow-up there were fewer late deaths ( 0.5 vs. $1.3 \%)$, reoperation rates ( 1 vs. $1.4 \%)$, infective endocarditis ( 0.5 vs. $0.7 \%)$ and stroke ( 0 vs. $0.2 \%)$ in the mini-thoracotomy group. Conclusions: In our experience minithoracotomy has proved several clinical advantages over sternotomy: significative less bleeding and transfusions, shorter ICU stay, less wound infections. Minithoracotomy is a less invasive procedure with similar mid term results.

\section{Degenerative Mitral Valve Disease: Does Mini-Thoracotomy Allow the Same Surgical Repair?}

\section{Marco Diena, Gheorghe Cerin, Bogdan Adrian Popa, Eugenio Novelli, Silvia Simonini, Angelo Romano, Levan Karazanishvili, Gabriele Musica \\ San Gaudenzio Clinic, Novara, Italy}

Objective: The key hole surgery is considered more cumbersome compared to sternotomy. We compared two groups of patients affected by degenerative mitral regurgitation in which the surgical repair was achieved through median sternotomy (Group 1) or right mini-thoracotomy (Group 2). We analysed the repairs from a technical point of view in order to verify whether a significant differences exist between the two approaches. Methods: Data from 585 patients who underwent mitral valve surgery from Jan'09 to Dec'13 were prospectively collected and analysed. For patients' characteristics see table 1 . Surgery: within the minimally invasive approach the cardiopulmonary bypass was achieved through direct ascending aorta cannulation and insertion of a percutaneous venous cannula in the groin. A flexible aortic cross clamp was applied through the skin incision and cardioplegic arrest was obtained with delivery of antegrade Custodiol ${ }^{\circledR}$ HTK solution. The surgical strategy aimed to correct the MR with a single orifice and to rebuild the
ToC using Gore-Tex chordae and annuloplasty. AML et lesion was addressed by reconstruction with polytetrafluoroethylene sutures. PML prolaps corrected by either quadrangular or triangular resection and/or neochordal implantation. An annuloplasty ring was implanted for each patient. Statistical analysis: in order to control the effect of possible confounding variables, a bivariate logistic regression using the propensity score of the surgical approach as regressor. Results: There were similar rates of leaflet resection, implantation of PTFE neo-chordae and use of annuloplasty rings within the study groups. For details see table 1. Conclusions: In our experience, minimally invasive MV repair for degenerative incompetence allows a complete repair even in complex cases. No significant differences could be demonstrated between the two approaches. We may conclude that in right mini-thoracotomy allows the same surgical repair with good clinical and echocardiographic outcome.

\section{Valve Surgery in Patients Under Twenty Years-Tanzania Experience}

Wambura B. Wandwi ${ }^{1}$, Elijar V. Ussiri ${ }^{1}$, Bashir Juma Nyangassa ${ }^{1}$, Evarist Magendo Nyawawa ${ }^{1}$, Ulisubisya Mpoki ${ }^{1}$, Edda Assel Vuhahula ${ }^{2}$

${ }^{1}$ Muhimbili National Hospital, Dar-es-Salaam, Tanzania;

${ }^{2}$ Muhimbili University of Health and Allied Sciences,

Dar-es-Salaam, Tanzania

Objective: Valve surgery in patients under twenty years for acquired valve lesions is rare. In areas endemic to rheumatic fever (RF) infection is commonly contacted during school age period and its cardiac squealer noted when they reach age of mid-twenty and above. Indications for valve surgery in under twenty years old patients in developing country Tanzania are evaluated. Methods: All patients who had valve surgery at Muhimbili National Hospital Tanzania from May 2008 to May 2013 were studied. Demographic data, indications for surgery and valve operated were recorded and data analyzed. Results: Ninety seven patients 62 (63.9\%) female and $35(36.1 \%)$ males had valve surgery with minimum age of nine years. Eighty patients (82.5\%) had Mitral valve pathology; regurgitation $60(61.9 \%)$ and stenosis $24(24.7 \%)$ respectively reesulting in Mitral valve repair 32 (33\%) and repalacents 38 (39\%) beeing similar. Rheumatic Heart Disease (RHD) was cause of valve function failure. Mechanical prosthetic valves were utilised in all replacents. The charts show diagnosis and surgery done. Conclusion: The use of mechanical prothesis in this age group (under twenty) particularly females pose coagulation challenge adulthood. In developing world with facility deprivation this is new problem created.

Table 1. Patient's characteristics and surgical techniques. $S=$ sternotomy. $M=$ mini-thoracotomy (for Abstract of $\underline{\text { Marco Diena }}$ )

\begin{tabular}{|c|c|c|c|c|c|c|c|c|c|c|c|c|c|}
\hline Group & Nr. & Male & Age & LVEF & $\begin{array}{l}\text { Leaflet } \\
\text { resection }\end{array}$ & $\begin{array}{l}\text { Quadr. } \\
\text { resection }\end{array}$ & $\begin{array}{l}\text { Triang. } \\
\text { resection }\end{array}$ & $\begin{array}{l}\text { G-tex } \\
\text { AML }\end{array}$ & G-tex PML & $\begin{array}{l}\text { G-tex } \\
\text { BOTH }\end{array}$ & $\begin{array}{l}\text { Annuloplasty } \\
\text { ring }\end{array}$ & $\begin{array}{l}\text { Complete } \\
\text { ring }\end{array}$ & $\begin{array}{l}\text { Incomplete } \\
\text { ring/ } \\
\text { other }\end{array}$ \\
\hline $1(\mathrm{~S})$ & 385 & $203(52.7)$ & $65.7 \pm 14$ & $56.5 \pm 10$ & $125(32.4)$ & $48(12.4)$ & $77(20)$ & $28(7.2)$ & $139(36.4)$ & $172(44.6)$ & $382(99.2)$ & $341(88.6)$ & $41(10.6)$ \\
\hline $2(\mathrm{M})$ & 200 & $138(69.1)$ & $55.7 \pm 12$ & $61.6 \pm 6$ & $107(54.1)$ & $21(10.5)$ & $86(43)$ & $11(5.5)$ & $100(50)$ & $81(40.5)$ & $200(100)$ & 195 (98.7) & $5(1.3)$ \\
\hline $\mathrm{p}$ & - & - & - & - & 0.17 & - & - & - & - & 0.10 & NS & & \\
\hline
\end{tabular}




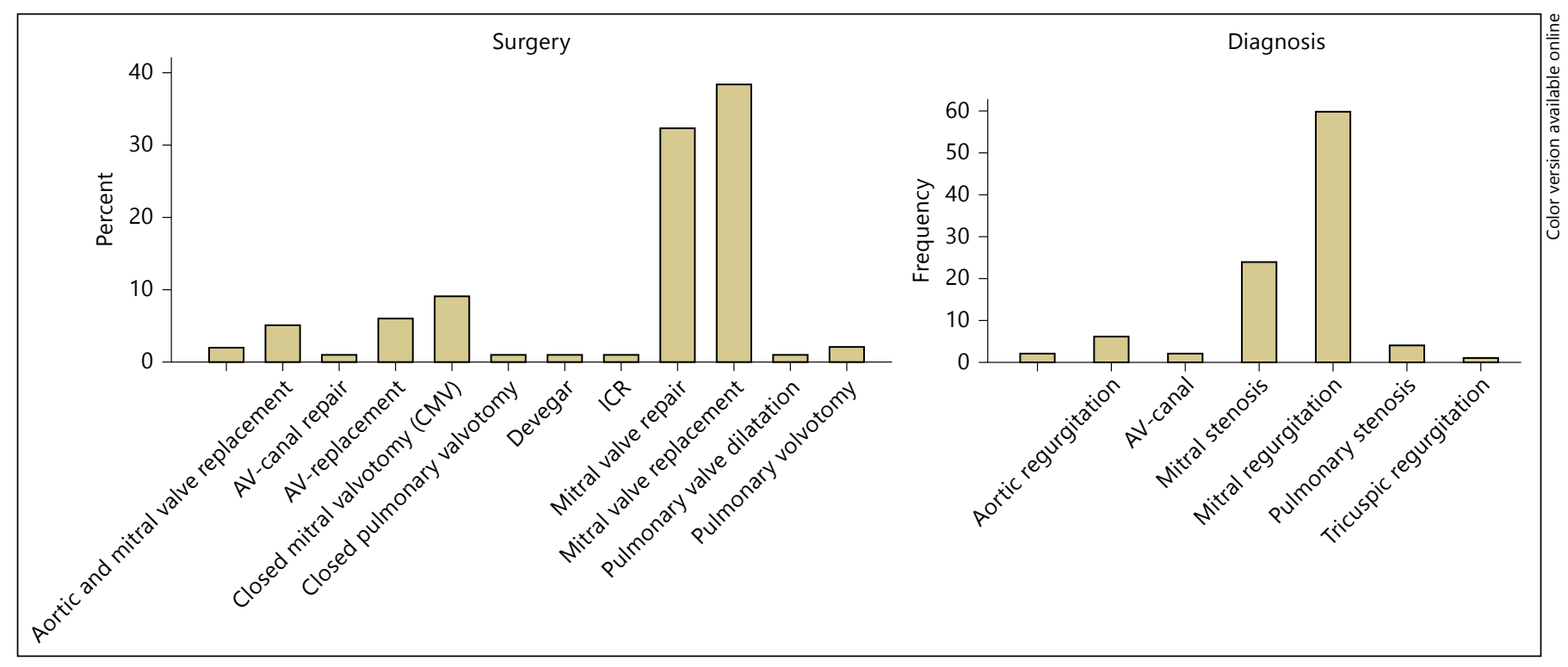

Fig. 1. (for Abstract of Wambura B. Wandwi).

\section{Partial Sterntomy in Valve Surgery}

Ehab Kasem, Sr., Ahamed Bakery, Ehab Sobhy

Zagazig University Hospital, Zagazig, Sharkia, Egypt

Obiective: Less invasive approaches have been popular since 1990, They have tha advantage over conventional sternotomyregarding post operative pain, less Blood required, less infection, less hospital stay and less coast, early result were less comparable to convential technique ding surgical exposure and high risk of strok nowadays, Results of partial sternotomy are comparable. Methods: From Jan 2011 to Jul 2013, 330 pt went for isolated valve surgery. we retro-spectively analysis our result in pt went for isolated valve surgery or combined with simple surgery 27 cases for upper sternotomy for Aortic valve replacement 33 pt went for partial lower sternotomy for isolated miteral valve or in association with ASD, Tricuspid valve disease Hower ever two case of miteral vale surgery went into Aortic valve and miteral valve surgery The discion of approach is individual. Results: The mean age in partial sternotomy (16-54) For full sternotomy (23-69) the intra-operative time Ischaemic time, partial sternotomy (31 \pm 7.2$)$ while in full sternotomy was $(37 \pm 6.7)$ total bypass time in partial sternotomy $(51 \pm 7)$ while in full sternotomy $(53 \pm 9)$ no of blood requrment in partial sternotomy $(275 \pm 35 \mathrm{ml})$ while in full sternotomy $(365 \pm 45)$ ventilation $\mathrm{Hrs}$ in partial sternotomy $(4.5 \pm 1.3)$ in ful sternotomy $(7.3 \pm 2.3)$ hospital stay partial sternotomy $(4 \pm 1.3)$ full sternotomy $(6 \pm 2.2)$. Conclusion: partial sternotomy in valve surgery is valuble technique and comparable to conventail sternotomy regarding pain, blood transfusion, ICU and Hospital stay and most important less surgical site infection earl rehabition and so no more cost.

\section{Timing of Valve Repair for Severe Degenerative Mitral Regurgitation and Long-Term Left Ventricular Function}

\author{
Yukikatsu Okada ${ }^{1}$, Yu Shomura², Takeshi Kitai ${ }^{3}$ \\ ${ }^{1}$ Midori Hospital, Kobe, Japan; ${ }^{2}$ Anjyo Kosei Hospital, Anjyo, \\ Japan; ${ }^{3}$ Kobe City Medical Center General Hospital, Kobe, \\ Japan
}

Objective: Current guidelines recommended surgery for patients with severe degenerative mitral regurgitation (MR) when specific left ventricular (LV) dimensions or ejection fraction (EF) are reached, based on the previous postoperative survival studies. This study aimed to evaluate the incidence and predictors of longterm postoperative LV dysfunction, and investigate the preoperative parameters necessarty to maintain or recover long-term LV function in the era of mitral valve (MV) repair. Methods: We retrospectively reviewed 473 consecutive patients undergoing MV repair for severe degenerative MR whom both preoperative and 3 -year postoperative echocardiographic data were available in our institution. Pre- and 3-year postoperative echocardiographic data and clinical outcomes were evaluated. Results: ROC analysis identified preoperative LVEF $\leq 63 \%$ (area under curve (AUC), 0.725; $\mathrm{p}<0.001$ ) and LV end-systolic dimension (ESD) $\geq 39 \mathrm{~mm}$ (AUC, $0.724 ; \mathrm{p}<0.001)$ as cut-off values for predicting LVEF $63 \%$ and LVESD $<39 \mathrm{~mm}$, while preoperative LVESD (odds ratio (OR), $2.22 ; \mathrm{p}=0.004)$, higher age $(\mathrm{OR}, 1.03 ; \mathrm{p}=0.04)$ and atrial fibrillation (OR, 2.68; $\mathrm{p}=0.01$ ) were independent predictors among patients with preoperative LVEF $\leq 63 \%$ or LVESD $\geq 39 \mathrm{~mm}$. Conclusions: Early MV repair with LVEF $>63 \%$ and LVESD $<39 \mathrm{~mm}$ well preserved long-term postoperative LV function, and smaller preoperative LVESD was associated with long-term LV function recovery, even in patients with preoperative LV dysfunction. 


\section{The Alternative Way to Reach to the Mitral Valve in Patients with Small Left Atrium; Superior Septal Approach}

\author{
Serkan Ketenciler ${ }^{1}$, Muslum Polat ${ }^{1}$, Murat Ercisli ${ }^{2}$, \\ Mehmet Kucukosmanoglu ${ }^{3}$, Gokalp Guzel ${ }^{1}$, Metin Kaplan ${ }^{4}$, \\ Birol Yamak ${ }^{2}$ \\ ${ }^{1}$ Gaziantep Dr. Ersin Arslan State Hospital Cardiovascular \\ Surgery Clinic, Gaziantep, Turkey; ${ }^{2}$ Adiyaman University \\ Medical Faculty Cardiovascular Surgery Department, \\ Adiyaman, Turkey; ${ }^{3}$ Gaziantep Dr. Ersin Arslan State Hospital \\ Cardiology Clinic, Gaziantep, Turkey; ${ }^{4}$ Gaziantep Dr. Ersin \\ Arslan State Hospital Anesthesiology Clinic, Gaziantep, Turkey
}

It is an important problem to reach the mitral valve for the patients that will be operated for the mitral valve pathology with small left atrium. In this study, we wanted to show superior septal approach is safe and alternative way to reach mitral valve for the cases that it is difficult to reach mitral valve by the standard way. Totally 227 patients that performed mitral valve replacement and/ or with additional procedures were added to study between 2001 and 2013. Isolated mitral valve replacement or concomitantly coronary artery bypass grafting, aortic valve replacement or tricuspid valve repair operation were performed to patients. The patients evaluated for transient or permanent rhythm disorders and early mortality. Transient rhythm disorders were seen $10 \%$ of patients but permanent rhythm disorder was detected only one patient. Hospital mortality was $3.6 \%$. To reach the mitral valve by the way of superior septal approach extends cross clemp time. When administered with caution it is not cause permanent rhythm disorders. We consider that superior septal approach for the patients that needed surgical mitral valve intervention with small left atrium is safe and alternative way.

\section{Plication Plasty for Reducing Posterior Mitral Leaflet Height}

\section{Aram K. Smolinsky}

Assuta Medical Center, Tel Aviv, Israel

Background: Excessive posterior mitral leaflet height is treated conventionally with sliding repair or folding plasty, both of which are irreversibly mutilating to the posterior leaflet. The plication plasty we introduce here is a non resection and reversible method. Method: Square pledgeted everting mattress sutures are employed along the rim of the P2 segment of the posterior leaflet, marked by the small commisures on its sides. Each stitch is commenced 2-3 mm away from the leaflet/ atrial wall border, in the atrial side. The needle points into the LV, penetrates back up through the posterior mitral leaflet $\sim 10 \mathrm{~mm}$ away from the rim, and later through the annuloplasty device. Once tied down, the mitral leaflet between the rim and the site of emergence of the needle will fold and be plicated between the pledget and the annuloplasty device. This fold at the base of P2 will reduce its height just as desired. The farther one aims the needle, the wider the plication. Excellent anchorage

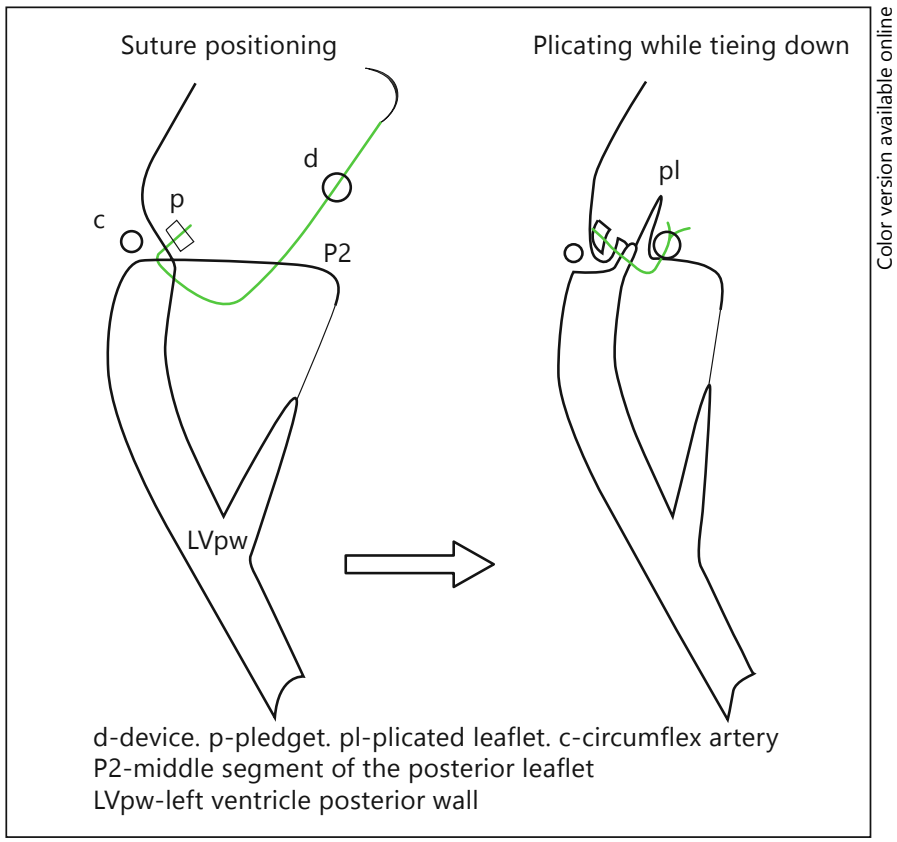

Fig. 1. (for Abstract of Aram K. Smolinsky).

of the ring to the posterior mitral annulus is a welcomed adjuvant. Results: This technique reduces the leaflet height in a controlled and reversible manner and strengthens the device anchorage at the posterior part of the mitral annulus. Near normal anatomy of the valve is maintained, both by operative appearance and the post-op TEE. The issue of post-repair SAM nearly vanished. Excellent anchorage of the area of the weakest ring tissue, at the base of P2, abolished the rare but troublesome cases of posterior ring dehiscence. Conclusion: Though insultingly simple, this approach for reducing posterior leaflet height provides efficient solutions for this issue, while strengthening posterior ring device anchorage as well.

\section{Anchor at 6 O'clock: The Most Secure Coupling of an Annuloplasty Device to the Posterior Ring}

\section{Aram K. Smolinsky \\ Assuta Medical Center, Tel Aviv, Israel}

Objective: The tissue at the posterior rim of the mitral valve is quite flimsy. Simple sutures there quite often feel unsafe. Yet, pledgeting the suture above the leaflet shortens its height. The chordae underneath the leaflet are commonly avoided as 'the dark forest thou shall not enter', because of the fear of catching chordae with the sutures. This presentation introduces a safe technique to anchor the annuloplasty device very securely just at the weakest and flimsiest point, just on 6 O'clock point, with a sub-leaflet/sub ring pledget, without interfering with chordae function. Methods: The 


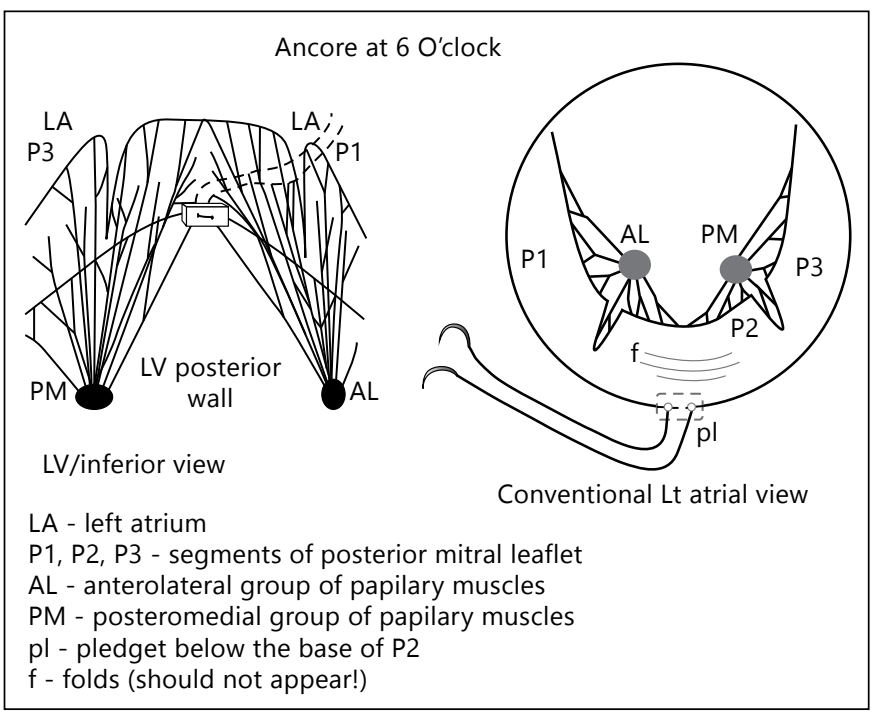

Fig. 1. (for Abstract of Aram K. Smolinsky).

chordae originate from two groups of papillary muscles. Those which originate from the antero-lateral group are attached to the $\mathrm{P} 1$ and lateral half of $\mathrm{P} 2$ segment. Those which originate from the postero-medial group are attached to the medial half of P2 and P3. Both groups are attached similarly to the anterior leaflet as well. One has to understand that the fields of attachments do not overlap. Thus there is a safe place just at the middle of the rim of the base of P2, free of chordae in the way of the sutures, which allow us safe positioning of a pledget there. To double check there is no such interference one has to note that while sliding the suture, no folds appear on P2, parallel to the rim ( $\mathrm{f}-$ in the figure). If they do, then one has to check and free sutures entangled in the pledget. Results: Our experience with this method exceeds 15 years. It nullified the phenomenon of early or late posterior dehiscence of annuloplasty device. It proved safe and nearly foolproof. Conclusions: By employing basic anatomic finding of chordae distribution, a single pledget can be safely positioned below the ring and leaflet, to provide the ultimate secure anchor at 6 O'clock.

\section{Efficacy of Nafamostat Mesilate as Anticoagulation During Cardiopulmonary Bypass on Early Surgery in Patients with Active Infective Endocarditis Complicated by Stroke}

Yutaka Okita, Toshihito Sakamoto, kenji Okada, Hiroya Kano, Katsuhiro Yamanaka, Shunsuke Miyahara, Takeshi Inoue, Naoto Izawa, Yasuko Gotake, Masamichi Matsumori

Kobe University, Kobe, Japan

Objective: Recent brain complications (bleeding or infarction) in patients with active infective endocarditis are recognized as a contraindication for early surgery if full heparinization is utilized.
Nafamostat mesilate (NM) is a synthetic protease-inhibiting agent which has not only potent inhibitory activity against coagulation factors (Xlla, Xa) but anti-inflammatory effect. We report our successful surgical experience using NM with low dose heparinization in patients with active infective endocarditis complicated by recent cerebral complications. Patients and Methods: Twenty seven patients with mean age of $54.9 \pm 18.7$ who underwent active infective native valve $(n=20)$ or prosthetic valve $(n=7)$ endocarditis were retrospectively reviewed ( 8 aortic, $16 \mathrm{mitral}, 2$ aortic/mitral, and 1 tricuspid). Twenty two of 27 patients were accompanied with preoperative stroke and 5 with active brain bleeding. Surgery was performed after the onset of stroke on $2.4 \pm 2.1$ days. NM $(209 \pm 152$ $\mathrm{mg})$ with low dose heparin $(3796 \pm 1218 \mathrm{IU}, 67.4 \pm 20.3 \mathrm{IU} / \mathrm{kg})$ was used for anticoagulation during cardiopulmonary bypass. ACT was maintained between 350 and 450 by fine administration of NM into a cardiotomy reservoir $(0.5 \mathrm{mg} / \mathrm{kg} / \mathrm{h})$ and a venous reservoir (sliding controlled dose at $1.5 \mathrm{mg} / \mathrm{kg} / \mathrm{h}$ ). Results: Cardiopulmonary bypass time was $181.3 \pm 92.6 \mathrm{~min}$. Five patients $(18.5 \%)$ died during hospitalization because of persistent sepsis in three and brain death caused by massive brain embolism before the establishment of cardiopulmonary bypass in one patient, and pneumonia in one patient. There was no further aggravation of intracranial bleeding or no new hemorrhage stroke. Conclusions: $\mathrm{Na}$ famostat mesilate with low dose heparinization might be useful for the early surgery in patients with active infective endocarditis complicated by stroke without the deterioration of the cerebral lesions.

\section{Self Modelling of Aortomitral Valves Will Enhance the Comprehension of Its Morphological Relationship}

Abdullrazak Hossien

Morriston University Hospital, Swansea, United Kingdom

Objectives: The aorto-mitral vicinity is of great clinical and surgical importance, because of its involvement in the anatomical and functional integrity of these two valves. We propose a multimedia learning guide for self-modelling of aorto-mitral valves to enhance the comprehension of its 3D complexity relationship. Methods: The left sided heart was constructed by usage available materials, in which its components was modelled according different atlases and heart specimens, then we established an effective multimedia learning guide describing the modelling steps of the left-sided heart. 7 cardiothoracic surgery trainees constructed the proposed model following the instructions of the proposed multimedia learning guide. The results reviewed by a senior surgeon. Results: Self Modelling of the left sided heart and its related structures results in improving of $3 \mathrm{D}$ understanding of the aorto-mitral valves relationship. The creation of the aortomitral angle leads to understanding the roll of this parameter in systolic anterior motion phenomenon. The trainees analyzed the architecture of the aortomitral continuity and its significance in mitral anuloplasty sizing. They identified the aortic root component and its relationship to mitral valve. Conclusion: The self-building of aortic root simulator under the direction of the multimedia guide results in improvement of comprehension of complexity of aortic root structures. 


\section{Flat-Plane AVR With Single Interrupted Sutures}

\section{Kazuo Tanemoto, Hiroshi Furukawa}

Kawasaki Medical School, Kurashiki, Japan

Background: While the aortic valve annulus has a scalloped shape, the sewing cuff of the prosthetic valve is nearly flat. Some cases of coronary orifice obstruction due to tissue valve have been reported. This is caused by force-fitting the flat prosthetic valve to the scalloped annulus of the aortic valve. Using our flat-plane AVR technique, we could achieve safe implantation, without distortion of the sinus of Valsalva. Methods: Our surgical technique involves placing single interrupted sutures and keeping the suture line flat to fit the prosthetic valve, and avoid changing the shape of the sinus of Valsalva / blocking the coronary orifice. At each of the three commissures, it is necessary to suture, adopting the 2-0 polyester double-armed mattress suture method using reinforced materials, from outside the sinus of Valsalva beneath the commissure, in order to create a flat suture line. Then, 21 interrupted sutures are placed (figure). We compared the balance (prosthetic valve size used minus the annular diameter assessed by preoperative echocardiography) achieved by this technique with that in cases operated by the conventional mattress suture technique. Results: A total of 43 cases were operated upon using this technique. The mean balance was $1.5 \pm 0.2 \mathrm{~mm}$, which was larger as compared with that $(0.5 \pm 0.2 \mathrm{~mm} ; \mathrm{p}=0.004)$ in cases treated by mattress suture technique. The postoperative $3 \mathrm{D}-\mathrm{CT}$ showed preserved shape of the sinus of Valsalva. Conclusion: We could resolve the discrepancy in shape between the aortic annulus and the prosthetic valves by placing the suture line in flat plane. Moreover, we could implant larger prosthetic valves. In cases with tissue valve failure in the future in which the valve-in-valve treatment is considered, the greater distance between the prosthetic valve and the coronary orifice obtained by this technique would be beneficial.

\section{Outcomes of a Minimally Invasive Right Anterior Thoracotomy Approach for Re-Operative Aortic Valve Surgery}

\section{Angelo LaPietra, Orlando Santana, Andres M. Pineda, Christos G. Mihos, Joseph Lamelas}

Mount Sinai Medical Center, Miami Beach, FL, USA

Objective: We sought to evaluate the feasibility, safety, and short-term outcomes of patients who requiring re-operative aortic valve replacement performed via a minimally invasive right anterior thoracotomy approach. Methods: We retrospectively reviewed all the re-operative minimally invasive aortic valve replacements performed at our institution between January 1, 2009 and February 28, 2013. The operative times, intensive care unit and hospital lengths of stay, post-operative complications, and mortality were analyzed. Results: A total of 79 consecutive patients with aortic valve disease requiring re-operative aortic valve replacement performed via a minimally invasive right anterior thoracotomy approach were identified. The mean age was $75 \pm 9$ years, and
61 of the patients were males (77.2\%). The mean left ventricular ejection fraction was $51 \pm 12 \%$. Previous surgeries included: 61 (77.2\%) coronary artery bypass graft surgery, 17 (21.5\%) aortic valve replacement, 10 (12.7\%) mitral valve surgery, and $12(15.2 \%)$ combined coronary artery bypass graft and valve surgery. The median aortic cross clamp and cardiopulmonary bypass times were 96 minutes (IQR 84-125) and 144 minutes (IQR 121-178), respectively. The median intensive care unit and hospital lengths of stay were 45 hours (IQR 32-85) and 8 days (IQR 6-11), respectively. There were 2 cerebrovascular accidents $(2.25 \%), 3$ re-operations for bleeding (3.79\%) and no conversions to sternotomy. The overall 30 -day mortality was 5 (6.33\%). Conclusions: Minimally invasive re-operative aortic valve replacement via a right anterior thoracotomy approach can be performed safely with a low morbidity and mortality.

\section{Self-Seeding Heart Valve Replacement Solutions Based on Self-Assembling Peptide-Functionalized Acellular Pericardium}

\author{
Monica Dettin, Roberta Danesin, Sabrina Facciolo \\ Annj Zamuner, Laura lop, Michele Spina, Gino Gerosa \\ University of Padua, Padua, Italy
}

Objective: Current mechanical and biological prostheses for heart valve replacement offer good haemodynamic performance, but have significant limitations, such as lack of viability and inability of somatic adaptation, exposing already treated patients to high risk re-interventions. The goal of this project is the formulation of novel self-seeding valve substitutes with improved bioactive and biomechanical properties, by functionalizing decellularized scaffolds with self-assembling peptide (SAP) hydrogels. Methods: EAbuK SAPs were synthesized using Fmoc chemistry and solid phase approach. Hydrogels structure determination was carried out by Atomic Force Microscopy and Scanning Electron Microscopy analyses. Decellularized bovine pericardium was incubated with peptide solution in water for $24 \mathrm{hrs}$ at $37^{\circ} \mathrm{C}$, followed by PBS. Entrapment and distribution within interfibrillar spaces were visualized by means of fluorescence microscopy and quantified by HPLC after pericardium treatment with rhodamine-labelled peptides. Scaffold modifications in thickness, area and water content were evaluated before and after SAPs gelation. Biofunctionalized samples were submitted to in vitro tissue engineering experiments with human bone marrow-mesenchymal stem cells and macaque aortic valve interstitial cells. Results: SAPs showed a tendency to spontaneously adopt $\beta$-sheet conformation in monovalent cation solutions. Fluorescence microscope analyses showed widespread incorporation of SAPs in pericardium scaffolds. Interestingly no peptide release was revealed by HPLC in solution after extensive washing of SAPs-treated samples. An increase in thickness, mass and water content, concomitant to a reduction in area, were demonstrated in functionalized pericardium. Both seeded cells were able to adhere: further analyses on their differentiation are under study. Conclusions: In this preliminary study, we demonstrated the penetration and retention of SAPs into decellularized pericardium samples. The evaluation 
of peptide-induced modifications of samples' area and thickness are in agreement with water enrichment. Filler properties of SAPs can be used to design a new class of easy-to-prepare, self-seeding heart valve substitutes. In addition to the already demonstrated capacity of SAPs to increase cell adhesion and growth, possible drug or protein delivery, as well as covalent functionalization with bioactive molecules make these peptides very interesting in cardiovascular tissue engineering applications.

\section{The Sliding Ruler Retrograde Measurements of the Mitral Valve Dimensions for a 3-D Optimal Geometry Annuloplasty Device}

\section{Aram K. Smolinsky}

Assuta Medical Center, Tel Aviv, Israel

Objectives: The conventional mitral valve repair measurements are based on standard sizers, one for each inter-trigone distance (ITD). 'Excessive' posterior leaflet is trimmed to fit. A measurement logic meant to account separately for all the individual valve dimensions: height, width, coaptation height and coaptation point depth is introduced. It is based on the anatomic findings that the base of P2 is equal to the ITD, throughout the growth of the normal hearts, opposite to the common findings of dilated posterior ring in the hearts with mitral regurgitation. Methods: The retrograde sliding ruler measurements; the end point of previous measurement is the zero point for the next. The point at the middle of the base of P2, known as the 6 O'clock point, is the reference starting point. The zero point of the device is its middle, and is coupled to the 6 O'clock point. This segment of the device should be preferably rigid. Next the two margins of the base of P2 are coupled to the device so as to be ITD apart. This becomes the new zero point for the next measurement, the AP diameter. The flexible lateral extensions of the device are adjusted and coupled to P1 and P3. Their measurement has to allow the extra length needed for the coaptation length and depth. The anterior completion to full ring is optional. Redundant device length is chopped off. In no case is the height of the posterior leaflet reduced. Results: This measurement logic allows a singe size device size which still accommodate

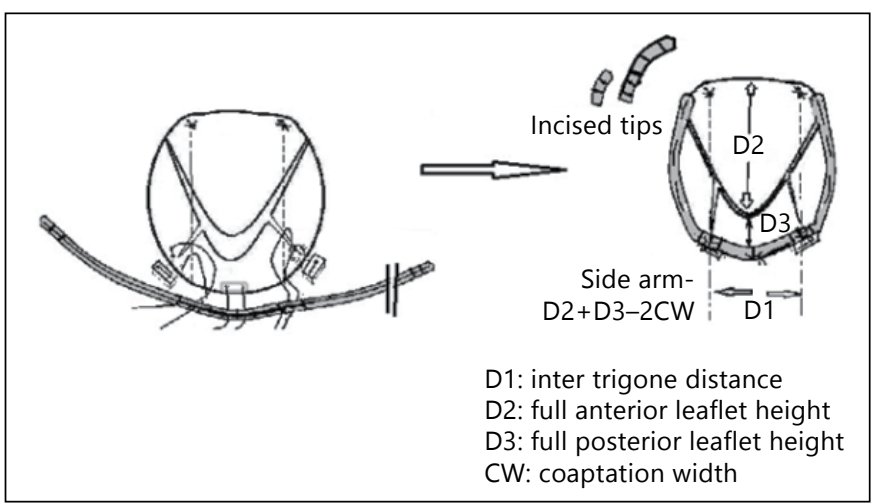

Fig. 1. (for Abstract of Aram K. Smolinsky). the particular patient leaflets geometry. There is no generalization: It is a single size custom measured and fit. There is no mitral valve area reduction and no space for SAM. Conclusion: Every mitral valve has its own individual dimensions. With the retro-grade sliding ruler anatomically adjusted measurements, one employs the same basic device for all repairs, yet accomplishes the optimal annuloplasty size and geometry for each specific valve.

\section{Should the Width of the Base of P2 Be a Corner Stone for Mitral Valve Ring Annuloplasty?}

Aram K. Smolinsky

Assuta Medical Center, Tel Aviv, Israel

Objective: The width of P2 (at its base) is not considered a clue to the understanding the underlining pathology of mitral valve regurgitation. One could assume that as the heart enlarges during the animal growth, the posterior ring/ $\mathrm{P} 2$ width will take a bigger share of it. We chose the model of the growing piglets. Not only is their heart considered to be very similar to humans, but within a year they weigh many times more. Our aim was to determine whether in the normal hearts, the heart growth influences the valve symmetry. Methods: Nineteen newborn piglets and six pigs form the basis for the measurements. The animals were weighed; their hearts were carefully dissected under operative microscope and weight separately. The measured parameters are the Inter trigone distance, (ITD) and the width of the base of P2, (WP2). They were compared to the same measurements in adult pigs. No animal was sacrificed for the purpose of this study. Results: Results and ratio are presented in the table 1 . Conclusion: In the normal porcine heart, through times of tremendous growth, there is no significant change in valve symmetry. The width of the base of $\mathrm{P} 2$ segment of the mitral valve annulus maintains a constant ratio to the inter trigone distance, of around 0.9. As the common surgical findings in repairing the dilated hearts with mitral insufficiency is an enlarged posterior ring, one may conclude that shortening this segment to the ITD should be a major issue of the ring annuloplasty.

Table 1. Heart growth and valve symmetry (for Abstract of $\underline{\text { Aram }}$ K. Smolinsky)

\begin{tabular}{|c|c|c|c|c|}
\hline & No. & $\begin{array}{l}\text { Body } \\
\text { weight } \\
\text { mean }(\mathrm{Kg})\end{array}$ & $\begin{array}{l}\text { Heart } \\
\text { weight } \\
\text { mean (gr) }\end{array}$ & $\begin{array}{l}\text { WP2/ITD } \\
\text { mean (SD) }\end{array}$ \\
\hline Piglet(newborn) & 19 & 1.133 & 7.93 & $0.85(0.09)$ \\
\hline Pigs & 6 & 320 & 425 & $0.93(0.11)$ \\
\hline Times growth & & 282 & 53.6 & \\
\hline P non-paired t-test & & & & NS \\
\hline
\end{tabular}




\section{Adverse Aortic Events After Isolated Aortic Valve Replacement for Bicuspid Aortic Valve Insufficiency and Concomitant Aortic Root Dilation}

Evaldas Girdauskas ${ }^{1}$, Kushtrim Disha', Mina Rouman ${ }^{1}$, Michael Andrew Borger ${ }^{2}$, Thomas Kuntze ${ }^{1}$

${ }^{1}$ Central Hospital Bad Berka, Bad Berka, Germany;

${ }^{2}$ Heart Center Leipzig, Leipzig, Germany

Objective: The optimal treatment of patients with distinct forms of bicuspid aortic valve (BAV) associated aortopathy is insufficiently defined. The aim of this study was to evaluate the risk of late aortic events after isolated aortic valve replacement (AVR) for BAV insufficiency with concomitant mild to moderate dilation of aortic root (i.e., root phenotype). Methods: Review of our institutional BAV database ( $\mathrm{n}=510$ patients) identified a subgroup of $56(11 \%)$ consecutive patients (mean age $47 \pm 11$ years, $95 \%$ men) with a BAV insufficiency and concomitant aortic root diameter of 40-50 mm (i.e., root phenotype), who underwent isolated AVR from 1995 through 2008. All these patients had a significant aortic annular dilation (i.e., defined as valve prosthesis size $\geq 27 \mathrm{~mm}$ ) and showed no relevant transvalvular gradient (i.e., mean pressure gradient $\leq 20$ $\mathrm{mm} \mathrm{Hg}$ ). All cases of simultaneous aortic surgery (i.e. with diameter of aortic root $>50 \mathrm{~mm}$ ) were excluded. Follow-up (516 patientyears) was $100 \%$ complete. Adverse aortic events were defined as the need for proximal aortic surgery / redo-AVR for late paravalvular leakage, the occurrence of aortic dissection/rupture, or sudden death during follow-up. Results: Actuarial survival rates of our study population were $91 \%$ and $78 \%$ at 10 and 15 years, respectively. Adverse aortic events occurred in 10 (18\%) study patients during follow-up. Proximal aortic surgery was required in 3 study patients (5\%) and three (5\%) additional patients underwent redo-AVR due to late paravalvular leakage. Two cases (4\%) of documented type A aortic dissection occurred. A total of 4 patients (7\%) suffered sudden cardiac death during follow-up. Freedom from adverse aortic events was $87 \%$ at 10 years and $47 \%$ at 15 years postoperatively. In a group of BAV patients presenting with aortic valve stenosis and concomitant mild to moderate dilation of tubular ascending aorta, freedom from adverse aortic events was significantly higher $(95 \%$ and $93 \%$ at 10 and 15 years, $p<0.001$ ). Conclusions: BAV patients with aortic valve insufficiency and concomitant aortic root dilation of 40-50 $\mathrm{mm}$ are at significant risk of adverse aortic events at 15 years after isolated AVR. Simultaneous aortic root surgery should be strongly considered in these BAV patients during their initial AVR.

\section{Newly Diagnosed Aortic Sclerosis Is Associated with Uncontrolled Hypertension: A Nested Case Control Study from the OXVALVE Population Cohort Study}

\author{
Margaret Loudon, Sean Coffey, Joanna d'Arcy, Andrew Kennedy, \\ Bernard Prendergast
}

Oxford University Hospitals NHS Trust, Oxford, United Kingdom

Objective: Aortic valve sclerosis (AVS) is associated with a 50\% excess risk of cardiovascular events and affects around $30 \%$ of

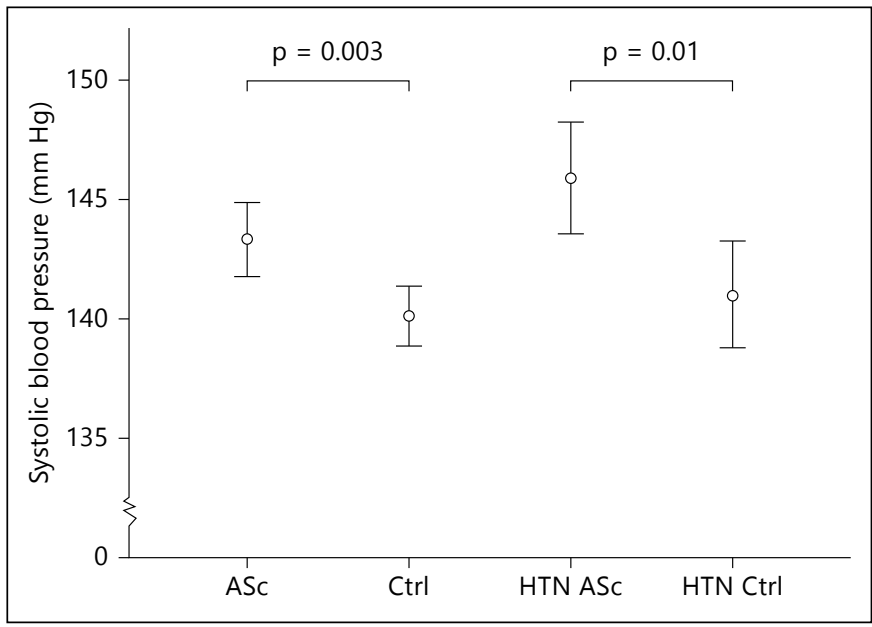

Fig. 1. (for Abstract of Sean Coffey).

those over 65 years of age. We examine the relationship between aortic sclerosis and hypertension in 600 patients with AVS identified within the larger OXVALVE population cohort study (OxVALVE-PCS). Methods: OxVALVE-PCS is a large ongoing, community based study in Oxfordshire, UK identifying newly diagnosed valvular heart disease (VHD) in subjects aged greater than 65 years. We compared the incidence and treatment of hypertension in the first 600 subjects with a new diagnosis of AVS identified on transthoracic echocardiography with nested controls within the larger OxVALVE population. Results: Systolic blood pressure was higher in those with a new diagnosis of AVS when compared with age matched controls without significant valvular heart disease (143.5 mm Hg vs. $140.1 \mathrm{~mm} \mathrm{Hg}, \mathrm{p}=0.003$ ) despite a similar proportion of previous diagnoses of hypertension (50.0\% vs. $47.6 \%$, $\mathrm{p}=0.41$ ). Importantly, when a cohort with known hypertension and AVS was compared to controls with known hypertension but no AVS, a persistent increase in systolic blood pressure remained (145.9 $\pm 20.5 \mathrm{~mm} \mathrm{Hg}$ versus $141.9 \pm 18.8 \mathrm{~mm} \mathrm{Hg}$; $\mathrm{p}=0.01$ ), despite similar levels of antihypertensive treatment. Conclusions: Aortic sclerosis is associated with more severe systolic hypertension when compared with age matched controls. This difference persists amongst those with known hypertension on treatment, suggesting therapeutic resistance. Mechanisms underlying this association and its pathophysiological explanation warrant further exploration followed by randomised controlled trials to investigate potential treatments to reduce the risks associated with this common condition. 


\section{Six-Year Outcomes After Non-Resective Mitral Valve Repair with Artificial Chordae Using Removable Clips}

\author{
Andrea Perrotti ${ }^{1}$, Chopard Romain ${ }^{1}$, Camille Durst ${ }^{1}$, \\ Lucian Stoica ${ }^{2}$, Nicolas Meneveau' ${ }^{1}$, Sidney Chocron ${ }^{1}$ \\ ${ }^{1}$ Hôpital Jean Minjoz, Besancon, France; \\ ${ }^{2}$ Institute for Cardio-Vascular Diseases 'Prof. Dr. George \\ I.M. Georgescu', lasi, Romania
}

Objective: We evaluated results of a non-resective technique for mitral valve repair with artificial chordae implantation that allows modification of the length of the chordae as many times as necessary, and avoids inadvertent alteration of chordal length during fixation by using removable clips. Methods: Once the length of the artificial chordae was determined, a removal clip was tied on the two thread ends to prevent sliding when performing the competence test. The length of the artificial chordae could be modified by opening the clip and closing it elsewhere on the artificial chordae. When the competence test was satisfactory, the threads were knotted on the clips. Long-term follow-up was completed between January and May 2012 by consultation plus echocardiography with a cardiologist of our institution. Results: 47 patients underwent repair with this technique. Follow-up was complete at a median of 6.0 years. There were no in-hospital deaths. Four deaths occurred during follow-up (8.5\%). The survival rate at 6 years was $91.5 \pm 2.1 \%$. One patient $(2.1 \%)$ had recurrent mitral regurgitation and required reoperation within 2 years after the initial surgery. Event-free survival rates, defined as survival free of death, reoperation, or cardiovascular adverse event was $87.2 \pm 5.2 \%$ at 6 years. At the time of follow-up, echocardiography found a fully competent valve or minimal mitral regurgitation in 45 patients (96\%), mild regurgitation in $1(2.1 \%)$ and significant mitral regurgitation in 1 patient $(2.1 \%)$. Conclusion: We report satisfactory 6 -year outcomes with our method using removable clips to determine the proper length of artificial chordae and to tie the knot accurately.

\section{Impact of Severe Pulmonary Hypertension on Outcomes Late After Aortic Valve Replacement for Aortic Stenosis Compared with Aortic Regurgitation}

\section{Luminita lliuta}

University of Medicine and Pharmacy 'Carol Davila', Bucharest, Romania

Objective: 1 . To evaluate the effect of aortic valve replacement (AVR) on pulmonary hypertension ( $\mathrm{PH}$ ) evolution, comparing patients with aortic stenosis (AS) to patients with aortic regurgitation (AR). 2. To determine whether PH remains a risk factor in the modern era for adverse outcomes after AVR for AS compared to AR. 3. To assess the independent predictors for persistence of the severe PH late after AVR. Methods: 5 years prospective study on 197 patients with severe $\mathrm{PH}$ undergoing AVR for AS (82 pts) or AR (115 pts) who were evaluated clinically and echocardiographically (including TDI) preoperatively and at 10 days, 1, 3, 6 months, 1 year and yearly 5 years postoperatively. Statistical analysis used SYSTAT and SPSS programs for regression analysis and relative risk calculations. Multivariable analyses were adjusted for age and gender and included left ventricular ejection fraction $\leq 35 \%$, renal insufficiency and logistic EuroSCORE $\geq 20 \%$. Primary endpoint was death of any cause within 5 years after AVR. Results: 1 . The evolution of the PH was different in AS group (early after AVR PH improved) compared with AR group. At 1 year postoperatively the percent of the patients with persistent severe $\mathrm{PH}$ was $21.95 \%$ in AS group and $56.52 \%$ in AR group. 2. At 5 years, cardiovascular event-free survival, including hospital visits caused by heart failure symptoms and sudden cardiac death was significantly higher in the patients with preoperative AS (59.76\%) compared with AR group (30.44\%). 3. Regression analysis identified as independent predictors for persistence of $\mathrm{PH}$ late after AVR: preoperative AR $(R R=21.2), E / E$ 'ratio $>12(R R=25.1)$, LA dimension index $>30 \mathrm{~mm} / \mathrm{m}^{2}(\mathrm{RR}=8.2, \mathrm{p}=0.0017)$, LV enddiastolic volume (LVEDV) $>200 \mathrm{~cm}^{3}(\mathrm{RR}=8.6)$, obstructive pulmonary disease $(R R=28.6)$, smoking $(R R=18.7)$ and 2 degree mitral regurgitstion $(M R)(R R=12.6)$. Conclusions: 1 . Severe $P H$ is reversible mostly after AVR for AS than for AR, both in the early and late postoperative term. 2 . The presence of severe $\mathrm{PH}$ had a significant impact on outcomes in patients undergoing AVR, decreasing long-term survival and increasing hospitalizations rates, mostly in those with preoperative AR. 3. The main predictors for persistence of severe PH late after AVR were: preoperative AR, E/E' > 12, LVEDV $>200 \mathrm{~cm}^{3}$, LA dimension index $>30 \mathrm{~mm} / \mathrm{m}^{2}$, obstructive pulmonary disease, smoking and associated 2 degree MR.

\section{Comparative Study of Standard Median Sternotomy (SMS) vs. Right Anterolateral Thoracotomy (RALT) for Mitral Valve Replacement}

Abdul Malik

Govt Lady Reading Hospital, Peshawar, Pakistan

Objective: To compare the outcome in mitral valve replacement done through standard median sternotomy versus right anterolateral thoracotomy. Methodology: Retrospective study from Jan 2009 to Dec 2011 at Department of Cardiovascular Surgery, Govt. Lady Reading Hospital Peshawar, Pakistan. Total 281 cases of mitral valve replacement (MVR) done among them 204 were operated through Standard Median Sternotomy (SMS) and 77 were operated through Right Anterolateral thoracotomy (RALT). Ethical committee approval was taken. An informed consent was taken for all patients. Age, sex, mortality, total cardiopulmonary bypass $\mathrm{CPB}$ time, time to establish CPB, mediastinal /chest drainage, post op blood transfusion, total hospital stay, ICU stay were analyzed and compared in the two groups. Statistical analysis done by SPSS version 17 and paired t test were applied to get $p$ value. $P$ value less than 0.05 was considered significant. Results: Females were predominant in both the groups (SMS 73.03\% and RALT $76.62 \%)$. Mean body surface area was 1.34 meter square. Mean age was 26.65 years in SMS and 26.42 years in RALT. There was no significant difference in mortality, cardiopulmonary bypass time, time to establish cardiopulmonary bypass, cross clamp time, ventilator time, chest drainage and ICU stay in the two groups. There was significant difference in post op blood transfusion and in total 
post op hospital stay. Conclusion: Sternem Sparing Mitral Valve Replacement can be done safely in selected cases. It gives better cosmetic results in females. RALT approach reduces hospital stay of patients and he can return to work early. Besides less pain, shorter skin incision and lower blood loss, it has more advantages as reduced sternal infection and sternal disruption.

\section{Simplified Myocardial Protection in Minimally Invasive Mitral Valve Surgery}

\author{
Yoshitsugu Nakamura ${ }^{1}$, Takaki Hori ${ }^{1}$, Takashi Murakami ${ }^{1}$, \\ Yujiro Ito ${ }^{1}$, Yujiro Hayashi ${ }^{1}$, Yohei Kawatani ${ }^{1}$, Hiroshi Niinami ${ }^{2}$, \\ Hiroyuki Nakajima² \\ ${ }^{1}$ Chibanishi general hospital, Chiba, Japan; ${ }^{2}$ Saitama Medical \\ University International Medical Center, Saitamai, Japan
}

Objective: In minimally invasive mitral valve surgery (MIMVS) via a right minithoracotomy approach, delivery of cardioplegia is relatively challenging because of technically demanding retrograde cardioplegia or troublesome changes of the mitral valve exposure for antegrade cardioplegia. Therefore we utilized crystalloid intracellular-type cardioplegia to diminish repeats of carioplegia delivery for MIMVS. This study reports its outcomes and efficacy. Methods: 27 patients underwent MIMVS using crystalloid intracellulartype cardioplegia from December 2011 to December 2013. The mean age was $59 \pm 11$ years old and 14 patients were male. All patients had moderate or severe mitral regurgitation Mean preopera- tive ejection fraction was $68 \pm 7$. The cardioplegia was delivered by antegradely through the aortic root cannula and the root cannula was disconnected from plegia-lines and left into the pleural cavity to save the working space. The second cardioplegia was given if arrest time prolonged over $150 \mathrm{~min}$. Results: All patients underwent mitral valve repair and mitral regurgitation improve to less than mild. Cardiac arrest time and cardiopulmonary time were $131 \pm 26$ min and $172 \pm 34 \mathrm{~min}$, respectively. The mean volume of cardioplegia was $2037 \pm 192 \mathrm{ml}$. Mitral valve repair was successfully completed in all patients. Only 1 patient $(4 \%)$ needed 2 nd delivery of cardioplegia. There was no failure to wean cardiopulmonary bypass. No hospital mortality was observed. The mean intubation time was 18.9 hours and postoperative creatine kinase $\mathrm{MB}$ was $30.1 \mu \mathrm{g} / \mathrm{ml}$. Mean postoperative ejection fraction was $60 \pm 12$. Conclusions: Simplified myocardial protection with crystalloid intracellular-type cardioplegia was feasible method for MIMVS.

\section{Total Reconstruction Of Ventriculo-Aortic Junction Using an Autologous Conduit and A Stentless Pericardial Aortic Bioprosthesis}

\section{Giuseppe Zattera, Pasquale Totaro, Alessandro Mazzola. \\ IRCC Foundation Hospital San Matteo, Pavia, Italy}

Objective: Treatment of acute infective endocarditis remains a surgical challenge expecially in presence of large disruption of the sub annular apparatus when a complete obliteration of the

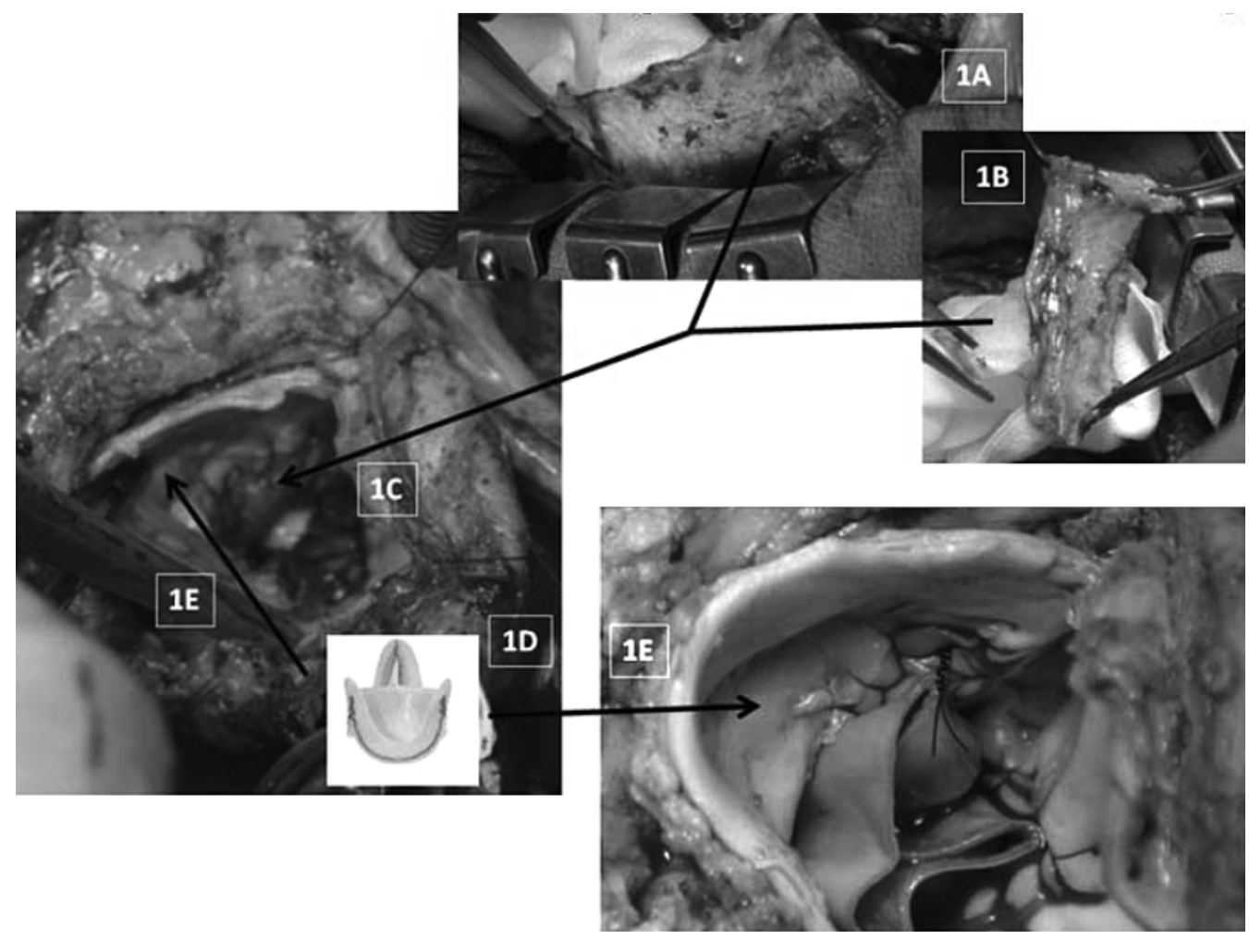

Fig. 1. (for Abstract of

Pasquale Totaro). 
abscess cavity before valve replacement is mandatory. Here we present an original technique which we have introduced for the treatment of acute endocarditis with complete disruption of ventrico-aortic junction (VAJ). Materials and Methods: Autologous conduit can be prepared using two different techniques: in the standard procedure a large patch of autologous pericardium is harvested following midline sternotomy. Free margins of the pericardial patch are then sutured together to create the cylinder of autologous conduit (fig la, b); Alternatively (ie. in case of REDO patients) the first portion of common pulmonary trank (without pulmonary artery) can be harvested and used as autologous conduit. Once the conduit is prepared, the two techniques are similar. Once the heart is arrested, aorta is opened and the valve excised. Subvalvular apparatus is inspected and the state of $\mathrm{VAJ}$ assessed. The VAJ is then reconstructed suturing the autologous cylinder below the area of disruption and above the aortic annulus (fig .1c). Reconsruction is completed with the implantation of a solo pericardial stentless bioprosthesis (fig. 1d) above the level of the distal anastomosis of the autologous cylinder (fig. 1e). Results: Both the described techniques allowed, in our series, complete AVJ reconstruction with full recovery in all patients. No case of postoperative complications related to the surgical technique were reported. Furthermore any recurrence of endocarditis was reported. Conclusions: Autologous reconstruction of ventriclolo-aortic junction is suitable and could improve postoperative outcomes. Such techniques allow indeed a complete debridment and exclusion of all the infected area leaving the supra-annular area intact where Solo pericardial bioprosthesis could be easily implanted.

\section{The Efficacy of Maze Procedure on Late Cardiac Function After Mitral Valve Repair for Mitral Regurgitation with Atrial Fibrillation}

\section{Yu Shomura, Yukikatsu Okada, Tadaaki Koyama, Naoto Fukunaga, Yasunobu Konishi}

Kobe City Medical Center General Hospital, Kobe, Japan

Objective: The advantage of Maze procedure concomitant with mitral valve (MV) repair remains unclear. The aim of this study was investigate the effect of Maze procedure on cardiac function in the patients underwent MV repair for degenerative mitral regurgitation (MR) with permanent atrial fibrillation (AF). Methods: We compared cardiac function 3 years after operation in 37 patients who underwent Maze procedure plus MV repair and converted to sinus rhythm (SR) postoperatively (Group M), with case-matched control patients who underwent MV repair alone and remained in SR postoperative (Group C). Matched variables were age, sex, preoperative left ventricular (LV) function (LV end-diastolic dimension (LVDd), LV endsystolic dimension (LVDs) and LV ejection fraction (EF)), and hypertension. Patients with more than moderate MR grade after operation were excluded from this comparison. Echocardiographic measurements were performed before and 3 years after operation in all patients. The LVEF and left atrial (LA) EF were measured by the biapical Simpson disk method. Results: The
Table 1. Echocardiographic data before and 3 years after operation (for Abstract of Yu Shomura)

\begin{tabular}{lccr}
\hline & Group M & Group C & $\mathrm{p}$ \\
\hline Preop LVDd & $5.7 \pm 0.7$ & $5.9 \pm 0.5$ & 0.1983 \\
Preop LVDs & $3.6 \pm 0.8$ & $3.6 \pm 0.6$ & 0.9243 \\
Preop LVEF & $60.4 \pm 9.0$ & $61.4 \pm 5.4$ & 0.5453 \\
Preop LA volume index & $126 \pm 67$ & $68 \pm 20$ & 0.0223 \\
3 years LVDd & $4.7 \pm 0.5$ & $4.6 \pm 0.5$ & 0.4594 \\
3 years LVDs & $3.0 \pm 0.6$ & $2.9 \pm 0.6$ & 0.6798 \\
3 years LVEF & $61.4 \pm 6.8$ & $58.2 \pm 6.4$ & 0.0439 \\
3 years LA volume index & $54 \pm 17$ & $41 \pm 18$ & 0.0025 \\
3 years LAEF & $24.3 \pm 10.6$ & $45.4 \pm 9.9$ & $<0.0001$ \\
\hline
\end{tabular}

preoperative and postoperative (3 year after operation) echocardiographic data were presented in table 1 . Three year after operation in both Groups, LVDd, LVDs, and LA volume index decreased significantly $(\mathrm{p}<0.0001, \mathrm{p}<0.0001$ and $\mathrm{p}<0.0001$ in Group $\mathrm{M}, \mathrm{p}<0.0001, \mathrm{p}<0.0001$ and $\mathrm{p}=0.0020$ in Group C). There was no significant difference between preoperative and postoperative LVEF in Group M. In contrast, the decrease was much greater for LVDd than LVDs in Group C; thus the LVEF decreased from $61.4 \pm 5.4 \%$ before operation to $58.2 \pm 6.4 \% 3$ year after operation $(\mathrm{p}=0.0116)$. Postoperative LAEF in Group $M$ was significant lower than that in Group C $(\mathrm{p}<0.0001)$. Conclusions: Correction of af with Maze procedure cannot improve the LA function equal to that in the patient with SR. However, It was suggested that restoration to SR after the Maze procedure with MV repair improve the LV function equal to or better than that in the patient with SR.

\section{Comparison of Miniinvasive and Full Sternotomy Implantation of New Suturelss Perceval S Aortic Valves}

Tomasz Niklewski, Krzysztof Filipiak, Michael Zembala, Roman Przybylski, Tomasz Kukulski, Marian Zembala

Silesian Center For Heart Diseases, Zabrze, Poland

Objective: There is an increase of old patients needing aortic Valve surgery. Especially in this age group, a lot of new less-invasive strategies are proposed. The Perceval S bioprosthesis (Sorin BiomedicaCardio) is a self-expanding valve designed to preserve aortic sinuses and sinotubular junction. We report the results of a the safety and efficacy of this stented bioprosthesis in patients undergoing aortic valve implantation with cardiopulmonary bypass and mini-invasive (partial upper sternotomy) and full sternotomy approach. Method: 32 patients were screened for Perceval S implantation 15 from mini and 17 from median sternotomy aproach. The mean diameter of the native aortic annulus measured In TEE before the operation was $21.9 \mathrm{~mm}$. Results: In the postoperative period most of the patients changed their NYHA class from III and II to II and I (72\%), with evident im- 
provement of exercise capacity. The mean transvalvular gradient of All group has changed significantly from $54.5 \mathrm{~mm} \mathrm{Hg}$ before the operation to $13 \mathrm{~mm} \mathrm{Hg}$ in follow-up. The ejection fraction (EF) was the same before and In follow-up echo: 54.4 and 55\%. The value of follow-up mean effective orifice area (EOA) calculated in follow-up transthoracic echo was $1.71 \mathrm{~cm}^{2}$, which is a very good value for biological aortic prostheses. Mean cross-clamp times (CCT) including patients who underwent surgery through mini and median sternotomy were, respectively, 54.2 and 49.9 minutes and and extracorporeal circulation times (ECT) 80 and 77.2 minutes $(p=N S)$. There was no significant difference between two groups in count of drainage and time of hospital stay. 31 patients alive in 6-36 months of observation. Only one patient of miniinvasive group died in hospital period because of intestinal ischemia followed the cardiac tamponade. Conclusions: Sutureless aortic bioprostheses bear the potential of easy implantation, reduced ischemic time, and surgical trauma in aortic valve replacement, is hemodynamically excellent prosthesis in selected patients. Due to a simple and fast implantation technique, this valve could guarantee a good operation time safety and efficiacy in combination with a miniinvasive approach.

\section{Analysis of Blood Haematological and Clinical Parameters in Patients with Implanted New Sutureless Aortic Perceval S Valves}

\author{
Tomasz Niklewski, Krzysztof Filipiak, Michael Zembala, \\ Tomasz Kukulski, Marian Zembala
}

Silesian Center for Heart Diseases, Zabrze, Poland

Objective: Sutureless implantation of Perceval S valves has a significant advantage over the classic technique, because of shortens aortic cross clamp time and the myocardial ischemia time, especially for elder patients with numerous comorbidities and small aortic annulus. This kind of valves start to be implanted in U.S. However the valve structure and it's similarity of biological part to SOLO bioprostheses lead us to analysis the influence on blood haematological laboratory paramethers and the clinical outcome during early follow-up. Methods: 32 Perceval S sutureless bioprosthetic valves were successfully implanted 15 implantations were made from mini and 17 from median sternotomy aproach. Mean age of patients was 74 years. Results: In the postoperative period most of 31 lived patients changed their NYHA class from III or II to I $(72 \%)$. With evident improvement of exercise apacity. 1 patient died because of cardiac tamponade complcations. The mean transvalvular gradient of All group has changed significantly from 54.5 $\mathrm{mm} \mathrm{Hg}$ before the operation to $13 \mathrm{~mm} \mathrm{Hg}$ in follow-up. In laboratory analysis of platelet count (PLT), value of hemoglobin (HB), hematocrit (HCT), reticulocytes and serum LDH before implantation, at discharge and in 6 months we observed the significant change of mean PLT from 212000 before to 195000 and 172000 in follow up. HB has dropped from 8.23 to 6.85 with increase to 7.88 (L/L), HCT from 39.0 to 32.7 and 38.2 (\%). Reticulocytes increased from 12.8 to 25.2 and dropped to 13.7 part-per-thousand, and serum $\mathrm{LDH}$ from 228.2 to 376.4 and 291.5 (U/L). We have reported 4 cases of anaemia with HB 5.9, 6.0, 6.2 and 6.2 (L/L) and 2 events of thrombocytopaenia with PLT 66000 and 88000 at discharge In 6 months follow up anaemia unresolved in 2 patients and thrombocytopaenia with PLT 94000 was still observed in 1 patient. Conclusions: We have concluded that Perceval $\mathrm{S}$ is a safe bioprosthesis that can easily be implanted, including by a minimalny invasive technique. It provides very good hemodynamic with significant clinical improvement. Overall, our data confirm the safety and utility of the Perceval bioprosthesis replacement with transitional changes of blood haematological values and low risk of thrombocytopaenia.

\section{Left Ventricular Apical Aneurysm and Sudden Cardiac Death in Hypertrophic Cardiomyopathy: A Tunisian Experience}

\author{
Abdeddayem Haggui, Mohamed Fares Mezni, Aymen Noomen, \\ Imen Hamdi, Houaida Mahfoudhi, Karima Taamallah, \\ Nizar Ben Mansour, Nadhem Hajlaoui, Wafa Fehri, \\ Habib Haouala \\ Military Hospital of Tunis, Tunis, Tunisia
}

Objective: Hypertrophic cardiomyopathy (HCM) is an extremely heterogeneous disease. An under recognized and very often missed subgroup within this broad spectrum concerns patients with left ventricular (LV) apical aneurysms in the absence of coronary artery disease. The aim of the present study was the evaluation of apical aneurysm as a new potential risk factor for sudden death in HCM. Methods: We hereby report a retrospective study about 48 patients with HCM followed between 1997 and 2013. Primary endpoint was life-threatening ventricular arrhythmias (LTVA) including SD, resuscitated SD and appropriate implantable cardioverter defibrillator therapy. The mean follow-up period was 5 years. Results: Our population was predominantly young at diagnosis with a mean age of $37 \pm 16$ years and most of them were male (65\%). At initial examination, maximal left ventricular wall thickness was $23 \pm 8 \mathrm{~mm}$, a LV outflow gradient $>30 \mathrm{~mm} \mathrm{Hg}$ was present in $23 \%$ and an apical aneurysm in $4 \%$ of patients. During the follow-up period, 12 patients (25\%) had LTVA. The annual rate of LTVA was $5 \%$. In our study, the overall probability of LTVA was significantly greater among patients with apical aneurysm than among those without $(\mathrm{p}=0.006)$. However, a multivariate analysis based on the Cox regression didn't identify apical aneurysm as a predictive factor of LTVA in our cohort study. Conclusions: Patients with LV apical aneurysms represent an underappreciated subset in the heterogeneous HCM disease spectrum with important clinical implications. Apical aneurysms in HCM are associated with a potential risk of sudden death and raise novel treatment consideration. 


\section{Left Ventricular Outflow Tract Obstruction and Sudden Cardiac Death in Hypertrophic Cardiomyopathy: A Tunisian Experience}

\author{
Mohamed Fares Mezni, Abdeddayem Haggui, Wafa Fehri, \\ Imen Hamdi, Houaida Mahfoudhi, Badii Jdaida, \\ Mehdi Ghommidh, Nizar Ben Mansour, Dhaker Lahidheb, \\ Nadhem Hajlaoui, Habib Haouala
}

Military Hospital of Tunis, Tunis, Tunisia

Objective: The effect of left ventricular outflow tract obstruction (LVOTO) at rest on the incidence of sudden death (SD) in patients with hypertrophic cardiomyopathy (HCM) remains unresolved. The aim of the present study was the evaluation of LVOTO at rest as a new potential risk factor for SD in HCM. Methods: We hereby report a retrospective study about 48 patients with HCM followed between 1997 and 2013. Primary endpoint was lifethreatening ventricular arrhythmias including SD, resuscitated SD and appropriate implantable cardioverter defibrillator therapy. The mean follow-up period was 5 years. Results: The 48 patients were predominantly young at diagnosis with a mean age of $37 \pm 16$ years and most of them were male (65\%). At initial examination, 11 patients $(23 \%)$ had peak instantaneous LVOTO gradients of $\geq 30 \mathrm{~mm} \mathrm{Hg}$ at rest. During the follow-up period, 12 patients (25\%) had life-threatening ventricular arrhythmias. The annual rate of life-threatening ventricular arrhythmias was 5\%. In our study, the overall probability of SD was not significantly greater among patients with outflow tract obstruction than among those without obstruction $(\mathrm{p}=0.251)$. A multivariate analysis based on the Cox regression confirmed that LVOTO was not independently associated with an increased risk of life-threatening ventricular arrhythmias in our cohort study. Conclusion: Our cohort study results do not support LVOTO as an independent risk factor for SD in patients with HCM.

\section{Medium-Term Results After Implant of a New Generation Stentless Pericardial Aortic Prosthesis: Hemodynamic Performance of Medtronic 3F at Rest and Under Exercise at 6 Years in 130 Patients}

\author{
Guglielmo Stefanelli, Sr., Fabrizio Pirro, Clorinda Labia, \\ Giuseppe D'Anniballe, Elisa Davolio, Giuseppe Gioia, \\ Giammarco Campoleoni
}

Hesperia Hospital, Modena, IT, Modena, Italy

Objective: Aim of this study was to evaluate the clinical and hemodynamic results at 6 years of Medtronic $3 \mathrm{~F}^{\circledR}$ stentless equine pericardial aortic valve. Methods: Between March 2007 and February 2013 a total of 130 consecutive patients affected by aortic valve disease received a $3 \mathrm{~F}$ valve at our unit. The size ranged between 21 and 29, with prevalence of 23 and 25 implants. Mean age at operation was $74.6 \pm 8.0$ years, $54 \%$ were males, the mean logistic EuroScore was $9.2 \pm 5.2$ and $47 \%$ received concomitant procedures. For isolated replacements the mean ECC time was 90' $\pm 10^{\prime}$, Cross Clamp time $72^{\prime} \pm 8^{\prime}$. In 14 pts the aortic prosthesis was included in a Dacron tube straight graft for a Bentall operation. Results: Early mortality in isolated AVR was $1.5 \%$ and $2.3 \%$ in the entire group. There have been 9 late all-causes deaths (4-non cardiac), with a survival of $93 \%$ at 6 yrs. $91 \%$ of patients are in NYHA class I or II. Actuarial freedom from reoperation for structural deterioration is $100 \%$ at 5 years.. Freedom from endocarditis and thromboembolic events were $98 \%$ and $100 \%$ respectively. The 72 patients evaluated under exercise at $75 \mathrm{w}$ protocol showed a moderate increase in the MPG (from $8.2 \pm 3.3 \mathrm{~mm} \mathrm{Hg}$ to $12.2 \pm 4.0 \mathrm{~mm} \mathrm{Hg}$ ) for the entire series. Conclusions: The $3 \mathrm{~F}$ valve shows excellent hemodynamics, durability and freedom from structural deterioration at 6 yrs follow-up. Freedom from endocarditis and thromboembolic events look also satisfactory. Longer follow-up times are needed for a better evaluation of this interesting new generation, user friendly aortic valve substitute.

\section{Degenerative Mitral Valve Repair: Presentation with Endocarditis Does Not Affect Long-Term Results}

Dan Spiegelstein, Dan Loberman, Michael Stein, Maor Yasmin, Leonid Sternik, Alexander Kogan, Micha S. Feinberg, Ehud Raanani

Chaim Sheba Medical Center, Ramat Gan, Israel

Objective: While the advantages of mitral valve repair over replacement are well documented for degenerative myxomatous disease, the comparative long-term clinical and echocardiographic outcome of degenerative mitral valve repair in patients with endocarditis is yet to be established. Methods: From 2004 to date, 555 patients with degenerative mitral valve underwent repair in our department. Of them, 43 (8\%) (SBE) presented for surgical repair: 27 with acute, 6 with sub-acute and 10 with healed mitral valve endocarditis. Their results were compared with the remaining 512 patients with degenerative mitral valve (non-SBE) who presented with other clinical manifestations. We prospectively followed all patients and analyzed outcomes regarding clinical improvement and echocardiography results. Results: There were no in-hospital deaths in SBE, and 2 in the non-SBE group (NS). Follow-up ranged to 9.2 years with a mean of $34 \pm 28$ months and $27 \pm 26$ months in SBE and non-SBE, respectively. Late all-cause mortality was 7\% (3 patients) in SBE versus $2 \%$ (10 patients) in non-SBE ( $\mathrm{p}=0.14)$. Freedom from re-operation on the mitral valve was $95 \%$ (2 patients) and 96\% (21 patients) in SBE and non-SBE, respectively $(\mathrm{p}=0.7)$. Freedom from recurrent endocarditis was $100 \%$ in the SBE group. Late echocardiography revealed that $95 \%$ and $91 \%$ of patients in SBE and non-SBE, respectively were free from moderate to severe mitral regurgitation $(\mathrm{p}=0.6)$. Freedom from NYHA FC III-IV was $94 \%$ and $88 \%$ in SBE and non-SBE, respectively ( $\mathrm{p}=$ 0.02 ). All other late valve-related complications were similar in both groups. Conclusions: Degenerative mitral valve repair presenting with infective endocarditis has similar mid- to long-term clinical and echocardiographic outcomes compared with non-endocarditis degenerative mitral valve. Recurrent SBE is rare. 


\section{Pre-Operative Enlarged Left Ventricle May Be a Risk Factor for Late Recurrent Mitral Regurgitation After Mitral Valve Repair Due to Left Ventricular Remodeling}

\section{Michael Stein, Hillit Cohen, Dan Spiegelstein, Leonid Sternik, Rafael Kuperstein, Micha S. Feinberg, Ehud Raanani}

Chaim Sheba Medical Center, Ramat Gan, Israel

Objectives: Mitral valve repair is now the first-line surgical approach for mitral regurgitation with well-documented advantages over mitral valve replacement. With its increasing use in recent years there have been more patients presenting with late repair failure, up to as much as $10-20 \%$ in most large series. Appreciating the specific mechanisms of mitral valve repair failure is crucial in optimizing surgical techniques and long-term repair durability. Methods: We prospectively followed 544 patients with degenerative mitral valve disease, who underwent primary mitral valve repair by a single surgical team between January 2004 and December 2012. Mean echocardiographic follow-up was 28 \pm 24 (range 1-95 months). At follow up, 41 patients (7.5\%) either needed reoperation due to recurrent mitral regurgitation (22 patients), or were found to have recurrent moderate or severe mitral regurgitation (19 patients). Clinical outcomes, re-operation reports and echocardiographic exams were analyzed and the mechanisms of repair failure were documented. Results: Freedom from reoperation on the mitral valve was $96 \%$. The primary mechanisms of repair failure were: recurrent mitral valve prolapse or flail in 23 patients (56\%), technical problems in 4 patients $(9.8 \%)$, sub-acute bacterial endocarditis in 3 patients $(7.3 \%)$, systolic anterior motion in 1 patient $(2.4 \%)$, restriction in 3 patients $(7.3 \%)$ and other mechanisms in 7 patients (17.1\%). Multivariate analysis found pre-operative pulmonary hypertension as the predictor for late valve failure $(p<0.01)$. Artificial chorda implantation was used in 306 patients, and of them we identified 17 patients $(5.5 \%)$ with valve repair failure, due to recurrent leaflet prolapse. Comparing the LVEDD and LVESD of these patients to all other cases with artificial chorda implantation, we found that failed cases tended to have greater pre-operative LVESD and LVEDD: $3.6 \pm 0.8$ vs. $3.3 \pm 0.6(\mathrm{p}=0.13)$ and $5.9 \pm 0.8$ vs. $5.6 \pm 0.6$, respectively $(\mathrm{p}=0.09)$. Conclusions: Recurrent mitral valve prolapse following primary mitral valve repair is a significant mode of failure that warrants further investigation. Enlarged pre-operative left ventricles with subsequent left ventricular remodeling post repair, may be a significant cause of repair failure, and requires careful attention when selecting the repair technique.

\section{Isolated Tricuspid Valve Surgery - Early and Late Outcome}

\section{Dan Spiegelstein, Hillit Cohen, Ido Ferstenfeld, Shani Levin, Leonid Sternik, Alexander Kogan, Ori Vatury, Rafael Kuperstein, Ehud Raanani}

Chaim Sheba Medical Center, Ramat Gan, Israel

Objective: Primary Tricuspid valve (TV) surgery is rare, as it is usually performed as a concomitant reconstruction procedure in addition to the correction of other cardiac pathologies, mainly mitral-valve surgery. The outcome for TV surgery as the primary indication is still unclear. We describe our experience of TV surgery, as TV pathology was the primary indication for surgery. Methods: From 2004 until 2013 we performed 569 TV surgeries. From this group we identified 55 patients, with TV pathology as the primary indication for surgery. Mean age was $61 \pm 14$ and $27 \%$ were males. TV pathology included RHD in 16 patients (29\%), secondary in $23(42 \%)$, endocarditis in $5(9 \%)$, prosthetic dysfunction in $5(9 \%)$, and other in 6 patients $(11 \%)$. Isolated TV was done in 49 patients (89\%) and there were 6 concomitant procedures: 4 CABG; 1 pacemaker and 1 PFO. From those 55 patients 18 underwent TV-repair (33\%) and 37 TVR (67\%). There were $70 \%$ redo operations. Results: Overall in-hospital mortality was 9\% (5 patients). Major complications included re-open for bleeding/tamponada $5(9 \%)$, prolonged ventilation (>48 h) $13(24 \%)$, respiratory failure and tracheostomy $3(5 \%)$, renal failure 7 (13\%), dialysis $10(18 \%)$, new pacemaker $3(6 \%)$, deep sternal wound infection 2 (4\%), and sepsis 8 (15\%). Mean hospital length of stay, ICU and ventilation times were $17 \pm 18,7.8 \pm 14$ and $2.2 \pm 3.5$ days, respectively. Multi-variant analysis for predictors of early mortality and major complication were NYHA FC IV \& female gender. During follow-up of $33 \pm 26$ months, there were 12 late mortality (24\%). Actuarial survival at 1, 2, 3 and 5 years were $78 \%, 73 \%, 71 \%$ and $66 \%$, respectively. Conclusions: Patients who require TV surgery constitute a high-risk group. There were no statistical differences in early and late outcomes between the isolated TV repairs versus replacement. Timely referral to surgery before the onset of class IV heart failure is recommended for improved outcome.

\section{Anterior Leaflet Extension}

\section{Phan V. Nguyen}

Heart Institute, Ho Chi Minh City, Vietnam

Objective: Severe leaflet retraction was presented in $15 \%$ of rheumatic MR. Leaflet extension with autologous pericardial patch was found a good solution to repair this lesion. Methods: Measurement the anterior leaflet diameter by TEE. If this diameter was less than $26 \mathrm{~mm}$. We need to extend the valve with pericardial patch. Results: From 1992 to 2008, 221 patients with rheumatic MR underwent leaflet augmentation technique at the Heart Institute - Viet Nam. Age ranged from 6 to 54 years old, $85 \%$ anterior leaflet extension and $15 \%$ posterior leaflet extension. The surface area of leaflet was increased up to $30-40 \%$ after extension. We can use the ring al least N.26 after extension. Hospital mortality $=2$ pts (1.2\%) Mean time of follow-up $108 \pm 2.61$ months. Echo control at the last examination: $77 \%$ excellent, $20 \%$ moderate MR and 3\% severe MR Actuarial survival rate at 10 years $=95.4 \pm 0.8 \%$ Non-fatal complcation: Endocarditis (4), patch dehiscence (2), leaflet retraction (4), calcified patch (18). 10 years free from reoperation $=96.4 \pm 3.6 \%$. Conclusions: This is a safe technique, increase feasibility of repair for rheumatic MR. Good solution for rheumatic MR and young children. 


\section{Clinical and Hemodynamic Evidence of Benefits and Feasibility After Implant of a Pericardial True Stentless Aortic Valve in $\mathbf{2 5 0}$ Consecutive Patients with 8 Years Follow-Up}

\author{
Guglielmo Stefanelli, Sr. ${ }^{1}$, Fabrizio Pirro ${ }^{1}$, Clorinda Labia ${ }^{1}$, \\ Giuseppe D'Anniballe ${ }^{1}$, Renata Biasin ${ }^{2}$, Andre Nyabenda ${ }^{3}$, \\ Massimo Longo ${ }^{1}$, Marco Meli ${ }^{1}$ \\ ${ }^{1}$ Hesperia Hospital, Modena, IT, Modena, Italy; ${ }^{2}$ Villa Berica \\ Hospital, Vicenza, Italy; ${ }^{3}$ Viila Berica Hospital, Vicenza, Italy
}

Objective: Aim of this study was to investigate the clinical and hemodynamic performance at rest and under stress of the Pericarbon Freedom Stentless (PF) aortic pericardial valve implanted on 250 patients, with up to 8 years follow-up. Methods: between March 2003 and April 2010, a PF stentless valve was implanted on 250 consecutive non selected patients (mean age: 68.7 yrs with $16 \%$ older than 80 years; $52.8 \%$ males). Mean EuroScore was $8.36-43.2 \%$ underwent concomitant procedures. Follow-up time ranged between 6 months and 8 years. Echocardiographic assessment was made at discharge, at 6 months and at last control. In a subgroup of 184 pts the hemodynamic performance was evaluated under moderate stress conditions. Results: Early mortality in isolated AVR was $0 \%, 1.2 \%$ in the entire group. There have been 23 late deaths, 19 non-cardiac, leading to 8 years survival of $84.1 \%$. Patients in NYHA class I and II were $90.9 \%$ at last follow up. Actuarial freedom from reoperation was $99.2 \%$ at 8 years, no structural valve deterioration has occurred. Freedom from prosthetic bacterial endocarditis was $98 \%$ at 8 yrs. The hemodynamic performance showed a mean pressure gradient of $8.4 \pm 4.5 \mathrm{~mm} \mathrm{Hg}$ at follow up, and an EOA of $1.85 \pm 0.7 \mathrm{~cm}^{2}$. The group evaluated under stress conditions showed a mild rise in mean gradients (from $7.8 \pm 3.3 \mathrm{~mm} \mathrm{Hg}$ to $11.6 \pm 4.0 \mathrm{~mm} \mathrm{Hg}$ ), yet associated to increased EOA (from $1.86 \pm 0.69 \mathrm{~cm}^{2}$ to $1.96 \pm 0.71$ $\mathrm{cm}^{2}$ ). Conclusions: The clinical performance of $\mathrm{PF}$ confirms favorable durability and freedom from events in the medium term. The PF showed also excellent hemodynamics along with slight increase of EOA under stress, indicating valve adaptability to different cardiac conditions and suggesting its use also for active, younger people.

\section{Tricuspid Valve Surgery in Patients with Isolated Tricuspid Valve Endocarditis - Analyzation of Perioperative Parameters and Long-Term Outcomes}

\section{Bettina Pfannmueller, Mareike Kahmann, Christian Binner,} Piroze Davierwala, Martin Misfeld, Jens Garbade, Pascal Dohmen, Christian Etz, Michael A. Borger, Friedrich W. Mohr

Heart Center Leipzig, Leipzig, Germany

Objective: Aim of this study was to investigate the perioperative conditions as well as short- and mid-term outcome of patients with isolated TV endocarditis undergoing TV surgery. Methods: Between June 1995 and February 2012, a total of 56 patients with isolated TV endocarditis underwent TV surgery at the Leipzig Heart Center. Mean age was $53.8 \pm 17.1$ years, mean LVEF $60.4 \pm 9.9 \%$, 39 patients $(69.6 \%)$ were male, 21 patients $(37.5 \%)$ suffered from renal failure, 13 patients $(23.2 \%)$ from diabetes mellitus. Average logEuroSCORE was $19.4 \pm 17.00 \%$. Follow-up was in average $5.4 \pm 3.9$ years. Results: Microbiological investigations of blood cultures and intraoperative material of the TV tissue/ vegetations were in 52 patients (92.9\%) positive and showed as the reason for TV endocarditis staphylococcus aureus in 24 patients (42.9\%) and coagulase negative staphylococcus in another 10 patients (17.9\%). The focus for developing TV endocarditis was in 19 patients (33.9\%) due to foreign material - especially pacemaker wires in 15 patients $(26.8 \%)$ - intravenous drug abusus was seen additionally in 11 patients (19.6\%). A TV-replacement was performed in 23 patients (39.3\%), TV repair with annuloplasty in 13 patients $(23.2 \%)$, in 21 patients (37.5\%) TV surgery was performed on the leaflet level (e.g. patchplastik, vegetatectomy). Overall 30 -day mortality was $12.7 \%$. Five year survival was $62.8 \%$ (95\% CI 5.3-11.5 years). The incidence for TV-related reoperation after 5 years was 7.5\% (95\% CI 6.4$8.7 \%$ ) and was in 4 of 5 patients due to TV-reendocarditis - two of these with recurrent intravenous drug-abusus, one patient with a pacemaker-wire and one patient with Re-Re TV endocarditis due to immunosuppression (acute leukaemia). Conclusions: TV surgery due to TV endocarditis often concerns patients with intravenous drug abusus, intravenous foreign material and/or patients with immunosuppression. Foreign material should be avoided in the case of TV-surgery so far as possible to diminish the risk of reendocarditis, especially in patients with intravenous drug-abusus.

\section{Mitra-Patch: An Expanded Poly-Tetrafluoro Ethylene (ePTFE) Patch Design To Simplify Mitral Valve Prolapse Repairs}

Muralidhar Padala', Weiwei Shi', Bryant Mclver', Jakob Vinten-Johansen ${ }^{1}$, Robert W.M. Frater', Surendra K. Chawla

${ }^{1}$ Emory University, Atlanta, GA, USA; ${ }^{2}$ Albert Einstein College of Medicine, New York, NY, USA; ${ }^{3}$ St. Francis Hospital, Hartford, CT, USA

Objective: Mitral neochordoplasty requires significant expertise in valve repair, to determine the optimal placement of chordae and neochordal length. We report a novel and simple ePTFE patch design (Mitra-Patch) with a muscle portion, a leaflet portion and multiple neochordae (fig a) that simplifies implantation without complicated sizing algorithms. Methods: The MitraPatch is laser cut from a radial reinforced ePTFE tube. Each patch consists of a web of four chordae, with proximal leaflet segments and a single papillary muscle attachment segment. Correction of cusp prolapse and regurgitation was tested in ex-vivo porcine hearts $(\mathrm{N}=6,3$ anterior and 3 posterior), then in five acute swine, with A2 prolapse from marginal chordal transection and in two chronic swine followed to 3 weeks and 4 months. The muscle portion of the device was sutured first to the papillary head with a special holder (fig b), followed by attachment to the leaflet edge 

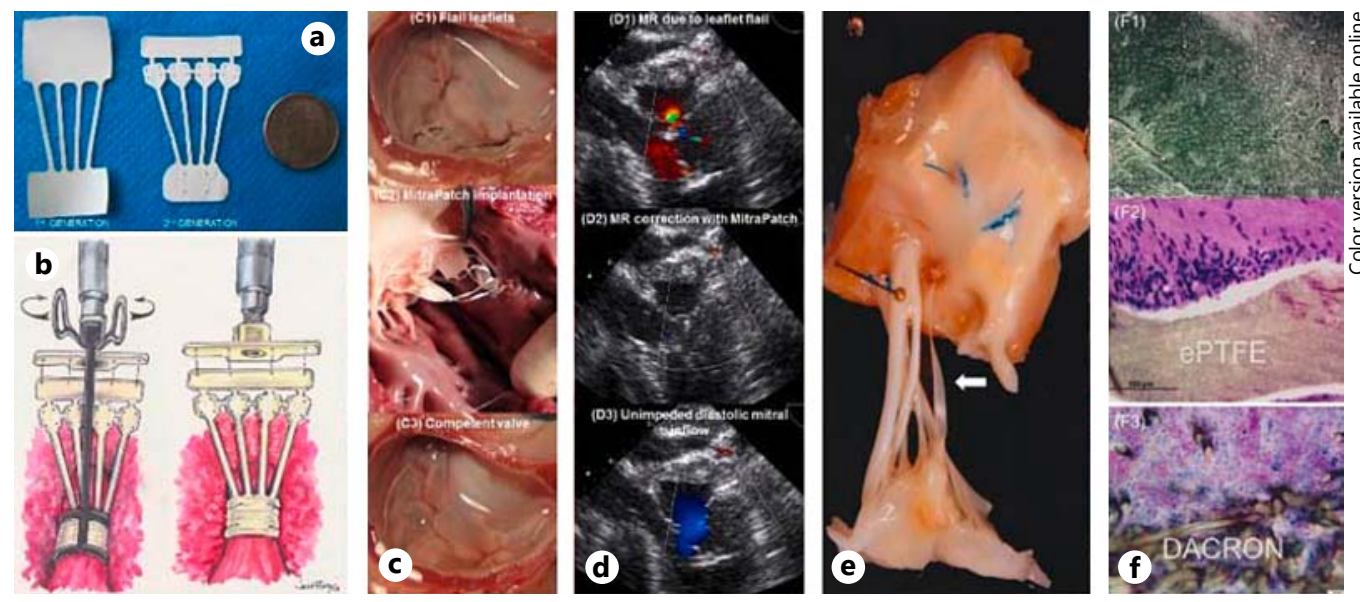

Fig. 1. (a) Evolution of the Mitra-Patch prototype from a single leaflet patch design to a multi-pronged arm design; (b) Mitra-Patch mounted on a special handle that enables its insertion and suturing to the papillary muscle head first, followed by its attachment to the leaflet edge at the prolapsing segment; (c1-2) Photographs demonstrating the efficacy of the Mitra-Patch in correcting mitral regurgitation in an ex vivo porcine heart induced with mitral leaflet flail; (d1-3) A representative echocardiograph in one animal with mitral regurgitation before repair of the mitral valve (d1), and at 120 days after repair of the valve with the patch, with good systolic closure (d2) and unimpeded diastolic filling (d3), (e) Photogragh of the patch after 120 days of implementation in the heart, depicting endithelialization and incorporation into the surrounding tissue; (f1) A scanning electron microscopy image of the patch depicting complete endothelialization of the surface at 120 days; (f2-f3) Healing between the patch and leaflet and the patch and the papillary muscle Dacron pledget (for Abstract of Muralidhar Padala).

using the length of the reference chord, which is the height of the stretched opposite leaflet chord. Echocardiographic imaging was performed at monthly intervals, with gross necropsy and detailed histopathological analysis. Results: Leaflet prolapse and regurgitation were repaired in all the ex-vivo hearts as shown in fig c1-3, with coaptation length restored from $0 \mathrm{~mm}$ before repair to $8.1 \pm 2.2 \mathrm{~mm}$ after posterior leaflet repair and to $10.2 \pm 1.3 \mathrm{~mm}$ after anterior leaflet repair. In the animals, regurgitation reduced from severe to trace (fig d1-3), that sustained over the follow-up period and without diastolic obstruction. Completed endothelialization was observed at 4 months by histopathology and surface electron microscopy (fig e). The patch was intact without evidence of stretch, degeneration, thrombus or fibrous tissue incorporation, and excellent endothelialization (fig f1), healing between the patch-leaflet (fig f2) and patch-muscle with Dacron pledget (fig f3) was clearly evident. Conclusions: The Mitra-Patch can effectively correct regurgitation in a swine model of leaflet prolapse. A one-time implantation of the papillary muscle segment followed by attachment to both the leaflets at the prolapsing segment simplifies the repair.

\section{Preoperative Pulmonary Function Tests as Predictors of Postoperative Morbidity and Mortality: A Multi-Institutional Analysis of 915 TAVR Patients}

\begin{abstract}
Amanda L. Maas ${ }^{1}$, Michael Mack², Gorav Ailawadi ${ }^{3}$, Rakesh M. Suri' ${ }^{4}$, Wilson Szeto ${ }^{5}$, Patrick Kilgo ${ }^{1}$, Todd Dewey ${ }^{2}$, Jacob R. Gillen ${ }^{3}$, John A. Kern ${ }^{3}$, Nimesh Desai ${ }^{5}$, Vuyisile T. Nkomo ${ }^{4}$, Rohan Menon ${ }^{5}$, Rebeca Kim ${ }^{2}$, Vinod H. Thourani ${ }^{1}$

${ }^{1}$ Emory University, Atlanta, GA, USA; ${ }^{2} \mathrm{CRSTI}$, Dallas, TX, USA;

${ }^{3}$ University of Virginia, Charlottesville, VA, USA; ${ }^{4}$ Mayo Clinic, Rochester, MN, USA; ${ }^{5}$ University of Pennsylvania, Philadelphia, PA, USA
\end{abstract}

Objective: Pulmonary dysfunction is a known risk factor for complications following conventional surgical AVR, but has not been well defined in the TAVR population. Currently, preoperative COPD severity is classified as mild, moderate, or severe using FEV1\%. This study sought to assess if preoperative PFTs or TAVR approach were predictive of pulmonary complications and short-term mortality. Methods: From 5/2008-3/2013, 915 patients with preoperative PFTs underwent TAVR for severe AS at 5 US academic institutions. Patients were stratified by COPD severity based on FEV1\% (no COPD: $>75 \%$, mild: $60-75 \%$, moderate: $50-59 \%$, severe: $<50 \%)$. Pulmonary morbidity and 30 -day mortality were analyzed in relation to PFT metrics (FEV1\%, FEV1/FVC\% and DLCO\%) and TAVR approach (transfemoral [TF], transapical [TA], or OTHER [transaortic, transaxillary, and trancarotid]). Results: For all patients, the mean age was 
Table 1. Postoperative mortality and pulmonary morbidity (for Abstract of Vinod H. Thourani)

\begin{tabular}{|c|c|c|c|c|c|c|c|c|c|c|c|c|c|c|c|}
\hline & \multicolumn{5}{|c|}{ COPD Severity* } & \multicolumn{3}{|c|}{ FEV1/FVC\%* } & \multicolumn{3}{|l|}{ DLCO\%* } & \multicolumn{4}{|c|}{ Approach* } \\
\hline & None $N=394$ & Mild N=202 & $\begin{array}{l}\text { Moderate } \\
\mathrm{N}=127\end{array}$ & $\begin{array}{l}\text { Severe } \\
\mathrm{N}=170\end{array}$ & $\mathrm{P}$ & $\begin{array}{l}>50 \\
\mathrm{~N}=865\end{array}$ & $\begin{array}{l}<50 \\
\mathrm{~N}=50\end{array}$ & $\mathrm{P}$ & $>50 \mathrm{~N}=591$ & $<50 \mathrm{~N}=211$ & $\mathrm{P}$ & $\begin{array}{l}\mathrm{TF} \\
\mathrm{N}=463\end{array}$ & $\begin{array}{l}\text { TA } \\
\mathrm{N}=338\end{array}$ & $\begin{array}{l}\text { OTHER } \\
\mathrm{N}=57\end{array}$ & $\mathrm{P}$ \\
\hline $\begin{array}{l}\text { Pneumonia, } \\
\text { n (\%) }\end{array}$ & $21(5.3)$ & $11(5.4)$ & $7(5.4)$ & $15(8.7)$ & 0.42 & $53(6.1)$ & $3(6.0)$ & 0.97 & $33(5.6)$ & $16(7.6)$ & 0.30 & $11(2.4)$ & $35(10.4)$ & $5(8.9)$ & $<0.001$ \\
\hline $\begin{array}{l}\text { Prolonged } \\
\text { vent, n (\%) }\end{array}$ & $47(11.8)$ & $29(14.3)$ & $13(10.1)$ & $17(9.9)$ & 0.53 & $98(11.3)$ & $7(14.0)$ & 0.56 & $60(10.2)$ & $31(14.7)$ & 0.07 & $34(7.3)$ & $58(17.2)$ & $6(10.5)$ & $<0.001$ \\
\hline $\begin{array}{l}\text { Vent hours, } \\
\text { mean } \pm \mathrm{SD}^{*}\end{array}$ & $43 \pm 141$ & $36 \pm 121$ & $33 \pm 117$ & $38 \pm 137$ & 0.93 & $39 \pm 135$ & $41 \pm 96$ & 0.91 & $34 \pm 133$ & $45 \pm 130$ & 0.38 & $21 \pm 70$ & $60 \pm 183$ & $24 \pm 61$ & 0.002 \\
\hline $\begin{array}{l}\text { 30-Day } \\
\text { Mortality, } \\
\text { n (\%)* }\end{array}$ & $23(5.8)$ & $8(4.0)$ & $6(4.7)$ & $10(5.9)$ & 0.76 & $45(5.3)$ & $3(6.3)$ & 0.77 & $23(4.0)$ & $16(7.7)$ & 0.032 & $12(2.6)$ & $25(7.4)$ & $5(8.8)$ & 0.004 \\
\hline
\end{tabular}

* Contains some missing data points.

COPD severity: note $=$ FEV $1 \%>75$, mild $=$ FEV $1 \% 60-75$, moderate $=$ FEV $1 \% 50-59$, severe $=$ FEV $1 \%<50$.

Prolonged vent $=>24 \mathrm{~h}$ mechanical ventilation .

$82 \pm 8$ years and mean STS PROM was $10.5 \% \pm 6.0 \%$. Based on FEV1\%, 44.1\% (394/893) had no COPD, 22.6\% (202/893) had mild, $14.2 \%$ (127/893) had moderate, and $19.1 \%(170 / 893)$ had severe COPD. TAVR approach was $54.0 \%(463 / 858) \mathrm{TF}, 39.4 \%$ (338/858) TA, and 6.6\% (57/858) OTHER. There were no differences in pulmonary outcomes across COPD classes or when FEV1/FVC\% and DLCO\% were dichotomized to $50 \%$. The only significant difference in 30-day mortality using these metrics was seen in the dichotomized DLCO\% comparison. When stratified by approach, incidence of pneumonia, prolonged ventilation, and ventilation time were highest in the TA group. 30-day mortality was lowest in the TF group when compared to the TA and OTHER cohort (table 1). Conclusions: Preoperative COPD is common among patients undergoing TAVR, however, the current classification utilizing FEV1\% was not useful in predicting 30-day mortality or pulmonary morbidity. Similarly, FEV1/ FVC\% was not predictive of these outcomes. Low DLCO\% was predictive of higher 30-day mortality rate, but not of postoperative pulmonary complications. Our findings suggest that COPD should not prohibit patients from undergoing TAVR and highlights that patients undergoing TA TAVR have the highest risk of pulmonary morbidity.

\section{Left Ventricular Outflow Reconstruction with the Pericarbon Freedom Stentless Bioprosthesis for Destructive Aortic Endocarditis}

Sandro Sponga, Cristian Daffarra, Deasy Pavoni, Igor Vendramin, Enzo Mazzaro, Giorgio Guzzi, Ugolino Livi

Udine University Hospital, Udine, Italy

Objective: The best valve substitute for valve endocarditis has not yet been identified. We report the results of the Pericarbon Freedom valve used to reconstruct the left ventricular outflow tract in destructive endocarditis of either aortic native valves or valve prostheses, complicated by periannular abscesses and root disarrangement. Methods: Since August 2007, 37 patients (mean age $68 \pm 12,76 \%$ males, Logistic Euroscore 19.6 \pm 8.8 , NYHA class $\geq 3$ in all cases) have undergone left ventricular outflow tract reconstruction with a Pericarbon Freedom stentless bioprosthesis. Seven patients (19\%) were in septic or cardiogenic shock preoperatively, 16 patients $(43 \%)$ suffered from moderate or severe aortic regurgitation. Ten patients (27\%) experienced preoperative systemic embolizations. Thirty-three cases (89\%) were valve redos and 8 patients $(22 \%)$ had concomitant procedures. Cusp perforations were found in 8 patients (22\%); discontinuity of the ventriculoaortal junction was detected in 11 patients (29\%) and in 32 patients (86\%) abscesses involved more than $2 / 3$ of the annulus; Mean follow-up was $26 \pm 23$ months. Results: One patient (3\%) died at 30-day because of multi organ failure and septic shock. Actuarial survival was $85 \pm 6 \%$ at 1 year, $76 \pm 8 \%$ at 3 and 5 years, respectively. Postoperatively, 10 patients $(27 \%)$ required pacemaker implantation for atrioventricular block and 20 patients (54\%) developed renal failure. One drug addicted patient (3\%) developed endocarditis relapse treated with antibiotic therapy, and 1 (3\%) showed a mild paraprosthetic aortic leak. No patient needed reoperation. At the last echocardiographic evaluation, mean gradient, peak gradient and left ventricular ejection fraction were $8.1 \pm 5.0 \mathrm{~mm} \mathrm{Hg}, 15.7 \pm 7.3$ $\mathrm{mm} \mathrm{Hg}$ and $63.2 \pm 9.6 \%$, respectively. Conclusions: The PericarbonFreedom prosthesis proves to be an excellent substitute in cases of destructive aortic valve endocarditis with good hemodynamic performances and low risk of relapses. It is promptly available in different sizes, easy to implant and its large inflow 'skirts' can be used to reproduce the result obtained with the autologus pericardium usually employed to cover the abcesses. The technique of inverting the prosthesis into the left ventricle seems to facilitate the surgical approach by allowing a deeper and better sealing running suture. 


\section{Aortic Valve Replacement with the Freedom Solo Stentless Valve: Nine Years Clinical and Hemodynamic Findings}

\author{
Sandro Sponga, Daisy Pavoni, Gaetano Nucifora, \\ Cristian Daffara, Igor Vendramin, Enzo Mazzaro, Daniela Piani, \\ Cristina Lutman, Ugolino Livi
}

Udine University Hospital, Udine, Italy

Objective: The Freedom Solo is a pericardial stentless valve implanted in supra-annular position with an easy running suture showing early good hemodynamic performance. The study aim was to evaluate the midterm results of this bioprosthesis. Methods: Between December 2004 and November 2009, 108 patients (31 males; mean age $78 \pm 6$ years) underwent AVR with the Freedom SOLO. Preoperatively the mean NYHA class was $2.5 \pm 0.7$ and the mean logistic Euroscore was 10 \pm 7 . Results: The mean prosthesis size was $22.7 \pm 1.9 \mathrm{~mm}$, concomitant procedures were performed in 65 patients $(60 \%)$. Two patient (1\%) died at 30 days because of intestinal infarction and respiratory failure respectively. Mean follow up was $69 \pm 17$ months and was completed in $100 \%$ of patients. One, 5 and 8 years actuarial survival was $88 \%, 73 \%$ and $66 \%$, actuarial freedom from structural valve deterioration was $99 \%, 91 \%, 79 \%$, actuarial freedom from reoperation $99 \%, 97 \%$ and $92 \%$, actuarial freedom from tromboembolic events was 99\%, 98\%, 96\% respectively. During echocardiographic follow up (mean $62 \pm 28$ months) data worsened mildly but progressively: discharge, 1 year, 3 years and 5 years mean gradients were $(8.4 \pm 3.6,9.1 \pm 4.0,10.5 \pm 3.6$, $11.3 \pm 4.4 \mathrm{~mm} \mathrm{Hg} \mathrm{p}<0.01$ ), indexed effective orifice areas were $\left(1.1 \pm 0.4,1.0 \pm 0.3,0.9 \pm 0.2,0.8 \pm 0.2 \mathrm{~cm}^{2} / \mathrm{m}^{2}, \mathrm{p}<0.01\right)$, left ventricular masses were $(213 \pm 61,186 \pm 60,199 \pm 61,205 \pm 68 \mathrm{~g}, \mathrm{p}=0.72)$. Transprosthetic regurgitation was absent in 92 (85\%) and trivial/ mild in $13(12 \%)$ and moderate in $3(3 \%)$. Nine patients developed paravalvolar leak (8.3\%); in all but one was trivial or mild. Structural valve deterioration was detected in 10 patients due to leaflet fibrosis combined with gross calcification in $9(8.3 \%)$ and to leaflet retraction with severe aortic regurgitation $1(1 \%)$. Conclusions: At nine years follow up Freedom SOLO stentless valve seems to show satisfactory results being an actractive option mainly in cases of small annulus, however the initial unexpected worsening of prosthesis performance needs further evaluation at longer follow up.

\section{Simple Repair for Mitral Regurgitation in Barlow Disease}

Sagit Ben Zekry, Dan Spiegelstein, Innon Lev, Leonid Sternik, Alexander Kogan, Rafi Kuperstein, Ehud Raanani

Sheba Medical Center, Tel Hashomer, Israel

Objective: Mitral valve (MV) repair for myxomatous disease with Barlow disease is challenging. Multi segment prolapse, excessive leaflets tissue and huge annulus require complex surgery with less optimal results. Thus, we present a simple technique for repair. Methods: Between the years 2005-2013, 560 patients with degenerative MV regurgitation underwent valve repair. Of them, seven- teen patients with Barlow disease underwent Ring-only repair (age $49 \pm 14$ years, $35 \%$ male). All of these patients had very large MV annulus and multi segment prolapse involving both leaflets. The unique feature in those patients was that the main regurgitant jet was central. The surgical technique included only implantation of a mitral annuloplasty ring (average ring size of $36 \pm 4 \mathrm{~mm}, 15 \mathrm{pa}$ tients with CE Physio, 1 patient with CE Classic, 1 patient with $\mathrm{CE}$ Cosgrove) in order to achieve adequate leaflets coaptation and abolish the regurgitation jet. Results: All patients presented with moderate-severe/severe mitral regurgitation (vena contracta $0.6 \pm 0.1 \mathrm{~cm}$, regurgitation volume $52 \pm 22 \mathrm{ml}$ ) with preserved ejection fraction $(58 \pm 22 \%)$. The mitral regurgitation jet was central in 12 patients while in the rest of the patients had combined central and eccentric jet. No flail was noted. Cardiopulmonary bypass and cross-clamp times were, $68 \pm 28$ and $52 \pm 19$ minutes. Post-repair mean coaptation length was $16 \pm 2 \mathrm{~mm}$. There was no in-hospital mortality. Hospital length of stay was $5.2 \pm 1.4$ days, where the only complication seen was transient atrial fibrillation in 3 patients. Mean follow up (FU) was $38 \pm 27$ months. There was no long term death as well as no need for reoperation during the follow up. Mean FU NYHA class is: $1.6 \pm 0.8$. Echocardiography FU revealed that all patients are free of moderate or severe MR. Conclusions: Mitral annuloplasty only for patients with severe multi segment myxomatous disease (Barlow), with central jet is safe, simple and reproducible with excellent mid term results. The post-operative long coaptation length may provide good long term results.

\section{EDWARDS INTUITY Valve System: An Innovative Solution for Minimally Invasive Aortic Valve Replacement}

\section{Francesca Chiaramonti, Marco Solinas, Federica Marchi, Tommaso Gasbarri, Sergio Berti, Mattia Glauber}

Heart Hospital G. Pasquinucci, Massa, Italy

Objective: The sutureless bioprosthesis represents an innovative approach for aortic valve replacement (AVR) reducing cardiopulmonary bypass (CPB) and aortic cross-clamp (ACC) times with a faster and easier implantation. These characteristics make it especially suitable for minimally invasive approaches (MIA). We present our initial experience with a new class of rapid-deployment aortic valves, the EDWARDS INTUITY Valve System (Edwards Lifesciences LLC, Irvine, Calif) implanted in MIA. Methods: Between June 2012 and December 2013, 47 patients with symptomatic aortic stenosis underwent AVR with EDWARDS INTUITY Valve. Of these 36 patients underwent isolated AVR. In 31 (66\% of all patients and $86 \%$ of isolate aortic valve disease patients) patients was performed an AVR in MIA: 21 (68\%) patients received an upper J-type minsternotomy and $10(32 \%)$ patients received a right anterior minithoracotomy. Follow-up was $100 \%$ completed (mean 9 months; range 1-18 months). Results: Implantation success was 100\%. Deployment bioprosthesis time was $15.3 \pm 4.1$ minutes. $\mathrm{CPB}$ and $\mathrm{ACC}$ time were $85.8 \pm 17.5$ and $52.2 \pm 13.8$ minutes respectively. These times are significantly lower then observed using sutured bioprosthesis in MIA. Mechanical ventilation time was 5.7 \pm 2.4 hours, ICU stay was $1.7 \pm 0.5$ days and ward stay was $5.7 \pm 1.4$ days. The transval- 
vulargradient at discharge was $10.2 \pm 4.1 \mathrm{~mm} \mathrm{Hg}$ (mean) and 16.6 \pm 6.4 $\mathrm{mm} \mathrm{Hg}$ (peak). At three months follow up the mean transvalvular gradient was $8.3 \pm 4.6 \mathrm{~mm} \mathrm{Hg}$ and the peak transvalvular gradient was 13.7 $\pm 7.2 \mathrm{~mm} \mathrm{Hg}$. There were no deaths. Conclusions: Minimally invasive AVR using an EDWARDS INTUITY Valve is a feasible and reproducible procedure with excellent results in terms of survival and hemodynamic performance at short term follow up. This bioprosthesis represent a useful tool that promise to reduce technical difficulties and operative times during AVR in MIA. Future evolutions of this device may performed a simpler and more easily reproducible implantation procedure, increasing the spreding of this approach. However, experience with a larger series of patients and a longer follow up are necessary to validate these preliminary data.

\section{Platform to Evaluate Aortic Root Repair Techniques Based on New Implantable Force Transducers}

Tommy Bechsgaard, Jesper L. Honge, Sofie Laugesen, Hans Nygaard, Sten L. Nielsen, Peter Johansen

Aarhus University Hospital, Aarhus N, Denmark

Objective: Surgical treatments for patients with aorta valve insufficiency secondary to aortic root dilatation (aneurisms) include the David reimplantation and the Yacoub remodeling technique. Even though these procedures were introduced more than 20 years ago, limited experimental research has been performed to document their acute effect. Therefore, the aim of this study is to develop a platform for a quantitative comparison of the biomechanical characteristics between the native healthy aortic root and the repaired roots. Methods: Two new force transducers are developed, one annulus transducer and one commissural transducer. The annulus transducer is placed just below the aortic valve and measures the dilating and contracting forces during the heart cycle. Wires are exteriorized from the heart past the mitral valve and the left atrium through a purse string suture. A total of six strain gauges are placed on the inside of the ring allowing for individual force measurements at each gauge point on the ring. The commissural transducer is a ring with three measuring rods placed equidistant. The transducer is placed upside down on the outside of the ascending aorta with the ring distal to the rods. Three strain gauges are mounted where the rods meet the ring. These provide measurements of the forces at each commissural point of the aortic valve. The transducers are calibrated under both static and dynamic settings. Results: The transducers have been manufactured using rapid prototyping in both titanium and hard plastic materials and strain gauges have been mounted. The linearity of the force transducers were tested by applying different known loads (0-5 Newton) to the transducer and plotting the output. A linear fit was made to the data yielding an Rsquared value of 0.9999 and a sensitivity of $184 \mathrm{mV} / \mathrm{N}$. Conclusions: Two aortic root transducers have been developed and characterized. These will provide the basic platform for characterizing various types of surgical procedures related to the aortic root.

\section{Enhanced Immunogenicity of Gal+ Bioprosthetic Heart Valves}

Guerard W. Byrne, Heide Kogelberg, Neha Passi, Christopher G.A. McGregor

University College London, London, United Kingdom

Objective: The objective of this study was to compare the immunogenicity of bioprosthetic heart valves (BHVs) produced from either wild type (WT) galactose alpha 1.3 galactose $(\mathrm{Gal})$ positive and Gal-free (GTKO) porcine heart valves in a clinically relevant nonhuman primate model. Commercial BHVs contain the major xenogeneic antigen $\mathrm{Gal}$ and patients treated with heart valve replacement show an induced anti-Gal antibody $(\mathrm{Ab})$ response. Methods: $\mathrm{Ba}$ boons had mitral valve replacement (MVR) with commercial BHVs produced from Gal+ $(n=4)$ and Gal-free $(\mathrm{GTKO})(\mathrm{n}=3)$ pigs. Recipients were immunized prior to BHV implant to increase anti-Gal $\mathrm{Ab}$ titers to human levels. Serum anti-Gal Ab levels were monitored by ELISA. Non- $\mathrm{Gal} \mathrm{Ab}$ responses were measured by Ab binding to GTKO porcine aortic endothelial cells (PAECs) using flow cytometry. Immunofluorescence microscopy was used to detect serum IgG binding to fixed GTKO porcine endothelial cells and to fresh frozen GTKO pig valves. Results: Immunization resulted in high anti-Gal IgG levels prior to BHV implantation in all recipients. Anti-Gal IgG levels fell rapidly after MVR in GTKO BHV recipients, returning to baseline in approximately 60 days. GT+BHV recipients maintained significantly elevated levels of anti-Gal IgG for at least the first year post implantation. GT $+\mathrm{BHV}$ recipients also showed increased IgG reactivity to GTKO pig endothelial cells 3 weeks after MVR which was not evident in GTKO recipients. Conclusions: Chronic elevated anti-Gal IgG levels during the first year post MVR in GT+BHV recipients, but not in GTKO BHV recipients is indicative of the expected increase Gal immunogenicity of glutaraldehyde fixed Gal+ BHV's. Increased IgG reactivity to GTKO endothelial cells further indicates that Gal+ BHVs also appear to have increased overall immunogenicity compared to GTKO BHVs. Enhanced immunogenicity of GT+ BHVs may be due to the adjuvant-like effect of the Gal epitope which can act to enhance immune presentation of non-Gal antigens. These data illustrate the range of immunogenicity of commercial Gal+ BHVs in a clinically relevant nonhuman primate model and support the hypothesis that more durable BHVs might be achieved using low antigenicity tissues from GTKO pigs.

\section{Late Results of Patients Undergoing Tricuspid Ring Annuloplasty for Functional Tricuspid Regurgitation in Mitral Valve Surgery}

\section{Naoto Fukunaga, Yukikatsu Okada, Yoshito Sakon, Yasunobu Konishi, Ken Nakamura, Takashi Murashita, Hideo Kanemitsu, Tadaaki Koyama}

Kobe City Medical Center General Hospital, Kobe, Japan

Objective: We reviewed 10-year experience to assess late survival and recurrence or progression of functional tricuspid regurgitation (TR) after tricuspid ring annuloplasty (TAP) for func- 
tional TR in the setting of mitral valve surgery. Methods: We retrospectively analyzed 220 patients who underwent TAP for functional TR concomitant with mitral valve repair (MVP; $\mathrm{n}=$ 160 ) or replacement (MVR; $n=60$ ) as an initial surgery from January 2000 to December 2010. Most frequent pathology in MVP was degenerative and in MVR was rheumatic mitral valve disease. The mean age was $65.4 \pm 11.4$ years and 107 male patients (48.6\%) were included. Sixty-one patients $(27.7 \%)$ were at New York Heart Association functional class III or IV (MVP; $57.3 \%$, MVR; 42.7\%). One-hundred and forty-seven patients (66.8\%) had preoperative atrial fibrillation (MVP; 58.8\%, MVR; 41.2\%). Preoperative degree of functional TR and right ventricular pressure were $1.9 \pm 0.9$ and $46.6 \pm 14.0 \mathrm{~mm} \mathrm{Hg}$, respectively. Left ventricular ejection fraction rate was $62.3 \pm 10.5 \%$. Indications of TAP at the time of mitral valve surgery included functional TR more than mild, a history of right heart failure, new onset of atrial fibrillation or pulmonary hypertension. The flexible ring or band was used forTAP. Mean follow-up period was $4.4 \pm 2.6$ years. Results: Hospital mortality rate was $5.5 \%(12 / 220)$. The size of flexible ring or band were $27 \mathrm{~mm}$ or $29 \mathrm{~mm}$ in most cases. Pre-discharge degree of TR was $0.7 \pm 0.6$ and TR of mild or less was identified in 04 patients (93\%). Overall survival at 5 and 10 years were $90.2 \% \pm 2.1 \%$ and $82.4 \% \pm 5.6 \%$, respectively. Late recurrent TR was defined as an increase in TR by greater than one grade and a final TR grade of more than mild. Freedom from late recurrent TR at 5 and 8 years were $87.7 \% \pm 4.2 \%$ and $78.0 \% \pm 6.6 \%$, respectively. A Predictor of late recurrent TR was elevated right ventricular pressure at final follow-up (HR, 1.091; $p=0.0003)$. Degree of TR at final follow-up was $0.9 \pm 0.7$. Conclusions: Late outcomes of patients who underwent TAP based on our strategy were acceptable. Late recurrent TR was associated with irreversible elevated right ventricular pressure.

\section{The Art of Documentation for TAVR Reimbursement}

\author{
Elizabeth K. Walsh, Wilson Szeto, Nimesh Desai, \\ Prashanth Vallabhajosyula, Saif Anwaruddin, Jay Giri, \\ Howard Herrmann, Joseph Bavaria \\ University of Pennsylvania, Philadelphia, PA, USA
}

Objective: On May 1, 2012 CMS (Centers for Medicare and Medicaid) and FDA (Food and Drug Administration) made a landmark decision by instituting mandatory national guidelines for aortic stenosis patients treated with TAVR (Transcatheter Aortic Valve Replacement). These rules would be used to enforce reimbursement for TAVR implants and to ensure that heart teams are trained and that patient outcomes would be tracked to better understand the benefit to the patient in real world outside of a trial. The purpose of this paper is to discuss the proper TAVR documentation to ensure compliance with the NCD (National Care Decision) guidelines. Methods: From November 15, 2007 through July 11, 2012308 TAVR patients' reimbursements were reviewed. The heart team also sent down with the hospital billing and coders to understand how the abstractors determined codes to be assigned. An expert heart failure physician was consulted to ensure the correct definition and the documentation being used by the team. Templates were designed so that key elements of documentation were not inadvertently omitted. Results: The findings of the audit revealed a need for consistent and frequent documentation throughout the patients chart. Symptoms of heart failure with evidence, treatment plan and outcomes must coincide Acute on chronic heart failure needs to be clearly stated along with at least one objective confirmatory result such as: elevated BNP (100 mg/ $\mathrm{ml}$ ) or NTproBNP (>1800 pg/ml), pulmonary congestion on chest $\mathrm{X}$-ray or physical exam demonstrating new volume overload to ensure that the patient will be coded with MCC (Major Complications and Co-Morbidities). Conclusions: With the implementation of the NCD, TAVR providers must take steps to ensure the documentation will hold up to scrutiny. Heart teams must audit

Table 1. Postoperative mortality and pulmonary morbidity (for Abstract of Elizabeth K. Walsh)

\begin{tabular}{|c|c|c|c|}
\hline \multicolumn{4}{|c|}{$\begin{array}{l}\text { Breakdown of MS-DRGs } \mathrm{N}=308 \\
\text { (DRG Weight } \mathrm{x} \text { Hospital base rate = reimbursement) }\end{array}$} \\
\hline MS-DRG/Name & Name & Medicare DRG Weight (2013) & Total \\
\hline 3 & Trach & 17.6369 units & $7(2.3 \%)$ \\
\hline 228 & Other Valves & 6.8682 units & $1(0.32 \%)$ \\
\hline 229 & Other Valve & 4.4413 units & $1(0.32 \%)$ \\
\hline 251 & Balloon Valuloplasty & 1.9737 units & $2(0.65 \%)$ \\
\hline 216 & Cardiac Valve w cath w MCC & 9.4801 units & $22(7.14 \%)$ \\
\hline 217 & Cardiac Valve w cath w CC & 6.2835 units & $4(1.3 \%)$ \\
\hline 219 & Cardiac Valve w/o cath w MCC & 7.9191 units & $168(54.5 \%)$ \\
\hline 220 & Cardiac Valve w/o cath w CC & 5.2917 units & $110(32.5 \%)$ \\
\hline 221 & Cardiac Valve w/o cath w/o MCC or CC & 4.6424 units & $3(0.97 \%)$ \\
\hline
\end{tabular}

MCC = Major Complications and Co-morbidities

$\mathrm{CC}=$ Complication and Co-morbidities

According to heart failure specialist, Mariell Jessup, MD, University of Pennsylvania: Acute on Chronic Heart Failure or Acute Heart Failure is applicable when at least one objective finding is made such as: Elevated BNP or NT-pro-BNP, evidence of pulmonoray congestion on chest $\mathrm{x}$-ray or volume overload with jugular venous distension, edema or ascites. 
their programs, communicate with their billing department and examine the documentation in the patient charts. A thorough investigation of the program will reveal discrepancies and educational needs so tools such as templates can be developed and tutelage can be provided.

\section{Aortic Valve Repair 10 Years Results: Sub-Groups Analysis}

\author{
Andrea Mangini', Monica Contino', Claudia Romagnoni', \\ Massimo Lemma ${ }^{1}$, Paolo Vanelli ${ }^{1}$, Guido Gelpi ${ }^{1}$, \\ Simone Colombo ${ }^{1}$, Carlo Antona ${ }^{2}$ \\ ${ }^{1} \mathrm{~L}$ sacco University Hospital, Milano, Italy; \\ ${ }^{2}$ Università degli Studi di Milano, Milano, Italy
}

Objective: In the last 20 years Aortic Valve Repair (AVR) has undergone a remarkable development so that now it represent an attractive alternative to aortic valve replacement; this process was due to a better comprehension of aortic root anatomy and of the pathogenetic mechanisms at the base of the aortic regurgitation. Now that AVR techniques have reached a good standardization level, we want to analyze our medium and long term results in relation to aortic regurgitation mechanism and different repair techniques adopted. Methods: From January 2003 to January 2013 we treated 218 patients affected by aortic valve regurgitation caused by leaflets pathology (prolapse, fibrosis or retraction) or root dilatation with a combination of the principal leaflet repair techniques and, when necessary, sparing procedures. Follow-up was achieved with periodic echocardiograms and clinical evaluations. We evaluated by means of Kaplan -Meier analysis the results in different subgroups of patients. Results: Freedom for $A R>2$ in patients with normal $(<25 \mathrm{~mm})$ virtual basal ring (VBR) was $92.5 \pm 3.3 \%$ while in patient with dilated VBR $(\geq 25 \mathrm{~mm})$ was $95.2 \pm 3.3 \%(\mathrm{p}=0.97)$. To analyse more clinically significant aspects of AVR, we clustered techniques to evaluate the most common and simple repair procedures: the functional aortic annulus (FAA) repair with replacement of the ascending aorta, or STJ plasty, associated to interleaflets triangles reshaping compared to complex procedures defined as an association of different techniques with or without sparing procedures. In this setting freedom from $A R>2$ was $98.0 \pm 1.9 \%$ for the simple procedures vs. $90.1 \pm 4.1 \%$ for complex procedures, $(\mathrm{p}=$ $0.15)$. Freedom from $A R>2$ in FAA repair with normal VBR $(<25 \mathrm{~mm})$ was $100 \%$ while in patient with dilated VBR $(\geq 25 \mathrm{~mm})$ was $90.0 \pm 9.5 \%(\mathrm{p}=0.04)$. We analysed freedom from $\mathrm{AR}>2$ in patients in which leaflets were repaired (plication, triangle resection or shaving): $89.5 \pm 4.4 \%$ compared to not touched $98.2 \pm 1.8 \%$ $(\mathrm{p}=0.11)$. Conclusions: All AVR techniques show good long term results if correctly selected in consideration of the native valve regurgitation mechanism. Surgical procedures involving significantly dilated VBR and/or important leaflets impairment are more challenging, but results are still satisfying.

\section{A Simple Method to Estimate Functional Aortic Annulus Dimensions}

\author{
Andrea Mangini, Monica Contino, Claudia Romagnoni, \\ Massimo Lemma, Guido Gelpi, Paolo Vanelli, Pietro Zerbi, \\ Carlo Antona \\ L sacco University Hospital, Milano, Italy
}

Objective: Sparing techniques and aortic valve repair procedures have changed the surgical view of the Aortic Root (AR). Features described by anatomist have been integrated by surgeons into their cultural burden, who now look at the aortic valve as a functional unit, in the form of a continuum embracing structure and function. Conservative aortic valve surgery is based on better understanding of aortic root anatomy and dynamics. From the analysis of anatomical relationships we can get some indications useful during aortic root surgeries, especially about functional aortic annulus. Methods: We photographed 16 formol-fixed human hearts with normal aortic roots to be analyzed and measured with a computer aided design software. Using Commissural Distance (CD) and Sinuses of Valsalva Nadir Distances (SVND) measures it was possible to esteem the virtual basal ring (VBR) and the Sino-Tubular Junction (STJ), representing respectively the inlet and the outlet diameter of the AR, both computed assuming their shape as circular. The estimated VBR (eVBR) was defined as the discrete circular distal boundary of the root where the valvular leaflet are peripherally attached. The estimated STJ (eSTJ) was defined as the circumscribed circumference of the triangle delimited by the apexes of the 3 commissures. To reduce the measurement errors due to the $2 \mathrm{D}$ configuration we averaged the value of the circumferences measured adding the 3 SVND (linear VBR-IVBR) and the 3 CD (linear STJ-1STJ) to the circumscribed circles calculated using the Heron's formula (cSTJ-cV$\mathrm{BR})$. This was possible after checking with the Carnot Theorem that the angles amplitudes were inferior to $90^{\circ}$. Diameters were calculated using the formula of the circumference $(\mathrm{C}=2 \pi \mathrm{r})$. Results: lVBR was $69.06 \pm 6.82$, cVBR $86.30 \pm 8.46$, 1STJ 76.67 \pm 7.69 and cSTJ $94.10 \pm+9.54$ so eVBR resulted $77.68 \pm 7.4$ and eSTJ $85.38 \pm 8.60$. The ratio between VBR and STJ was $1.11 \pm 0.17$. Conclusions: We propose a simple method to estimate the VBR and the STJ measures from the SVND and the CD lengths. This will help surgeons to better calculate the correct size of ascending aorta prosthesis and to standardize the reparative approach to the aortic root functional unit.

\section{Minimally Invasive, Off-Pump Placement of Artificial Chordae Tendinae to Correct Mitral Regurgitation: Initial Results of the European TACT Registry}

\author{
Richard Daly' ${ }^{1}$ Joerg Seeburger ${ }^{2}$, John Zentgraf ${ }^{3}$, John Seaberg ${ }^{3}$, \\ Giovanni Speziali ${ }^{3}$ \\ ${ }^{1}$ Mayo Clinic, Rochester, MN, USA; ${ }^{2}$ Heart Center Leipzig, \\ Leipzig, Germany; ${ }^{3}$ Neochord, Inc., Eden Prairie, MN, USA
}

Objective: The Transapical Artificial Chordae Tendinae (TACT) Trial completed enrollment in 2012 and results were recently reported. The trial evaluated a device that places artificial 
chordae tendinae (ACT) with transapical approach, off-pump with echo guidance. After completion of the TACT Trial, patient enrollment continued in the Registry, with a plan for a minimum of 75 patients. Methods: TACT Registry is a multi-center, prospective, and observational. Patients had $\geq 3+$ mitral regurgitation (MR) due to leaflet prolapse. Acute procedural success (APS), hospital discharge, 30 day and 60 day success were defined as reduction of MR to $\leq 2+$. Enrollment began in January 2013. To date, 30 patients have been enrolled and centers have provided echocardiographic data. Followup is planned at 30 days, 6 and 12 months. Results: 30 patients have been enrolled with data available. Characteristics include: mean age $62 \pm 16$ years, $70 \%$ male, mean LV ejection fraction $56 \pm 7 \%$, MR $3+$ in $70 \%$ and $4+$ in $30 \%$, NYHA class $1-2$ in $44 \%$ and class 3-4 in 55\%. Three patients had AC placed to anterior and 27 had AC placed to the posterior leaflet. AC placed per patient ranged from 2 to 6 , with a median of 3 . Average total procedure time was $135 \pm 34$ (75-220) minutes; average time to place all chords was $39 \pm 25$ (13-121) minutes APS was achieved in 29 of 30 (97\%); one patient proceeded to open mitral repair. Available data shows: success at hospital discharge in 23 of $25(92 \%)$, at 30 days in 12 of $14(86 \%)$, and at 6 months in 8 of 9 (89\%). The only patients with $\mathrm{MR}>2+$ at 30 days and 6 months were the patients that had $\mathrm{MR}>2+$ at hospital discharge. Conclusions: Placement of AC to treat severe MR due to leaflet prolapse using a transapical, off-pump approach resulted in APS in $97 \%$ of patients, with continued success in $89 \%$ of patients available for 6 month followup. All patient with successful correction of MR at hospital discharge continued to have success at 30 day and 6 month followup. Followup of these patients and Registry enrollment are ongoing.

\section{Annuloplasty Suture Forces: Preliminary Insight for Identifying the Mechanisms of Ring Dehiscence}

\author{
Eric L.Pierce ${ }^{1}$, Andrew W. Siefert ${ }^{2}$, Madonna Lee $^{3}$, \\ Chikashi Aoki ${ }^{3}$, Robert C. Gorman ${ }^{3}$, Joseph H. Gorman ${ }^{3}$, \\ Ajit P. Yoganathan ${ }^{1}$
}

${ }^{1}$ Georgia Institute of Technology and Emory University, Atlanta, GA, USA ${ }^{2}$ Georgia Institute of Technology, Atlanta, GA, USA; ${ }^{3}$ University of Pennsylvania, Philadelphia, PA, USA

Objective: An increasingly acknowledged short-term repair failure for functional mitral regurgitation is annuloplasty ring dehiscence. Though often attributed to surgical technique, no studies have identified whether suture failure, knot failure, or annular tissue tearing is the primary cause of dehiscence. To explore these mechanisms, this study aimed to demonstrate the first use of a novel technology to quantify suture forces for undersized mitral annuloplasty rings. Methods: Force transducers were developed and attached $(\mathrm{N}=10)$ to size 24 PhysioTM rings.

Fig. 1. (for Abstract of Eric $L$.

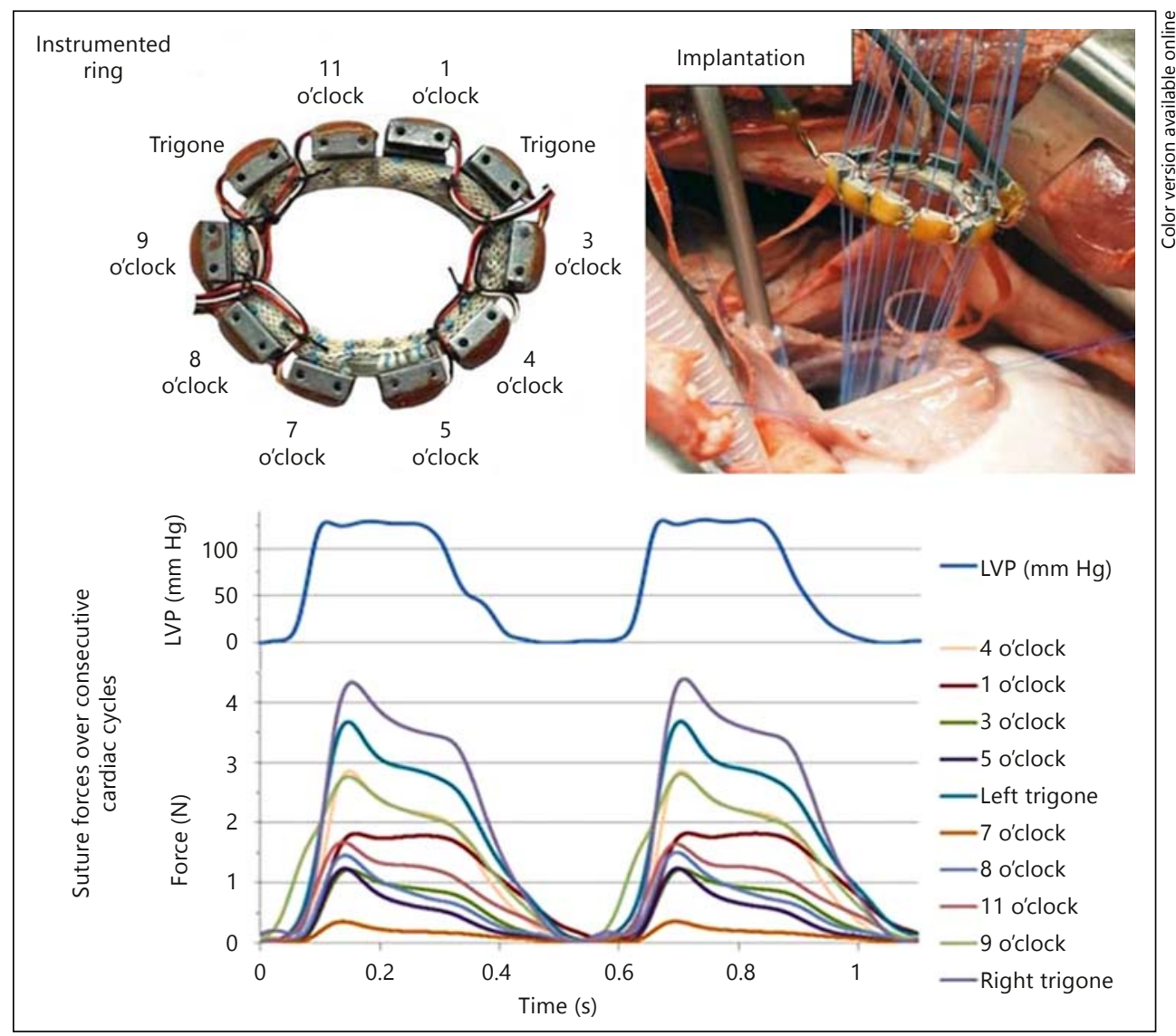


Under cardiopulmonary bypass, instrumented rings were implanted in the mitral annuli of healthy ovine animals $(\mathrm{N}=4)$. Each transducer was secured to the annulus with a single mattress suture by the exact process used in normal ring implantation. Post-cardiopulmonary bypass, suture forces were measured continuously over cardiac cycles reaching peak left ventricular pressures (LVP) of 100, 125, and $150 \mathrm{~mm} \mathrm{Hg}$. Results: The mitral annulus of each animal $(62 \pm 10 \mathrm{~kg})$ was successfully undersized by two ring sizes. Annuloplasty increased the diastolic transmitral pressure gradient by approximately $3 \mathrm{~mm} \mathrm{Hg}$ in each animal. Averaged across all sutures, cyclic forces (calculated as the difference between peak and minimum force over each cycle) were observed to increase from $1.6 \pm 1.1 \mathrm{~N}(100 \mathrm{~mm} \mathrm{Hg})$, to $1.9 \pm 1.2 \mathrm{~N}(125 \mathrm{~mm} \mathrm{Hg})$, to $2.0 \pm 1.4 \mathrm{~N}(150 \mathrm{~mm} \mathrm{Hg})$. At all levels of peak LVP, suture forces were greatest at the left and right fibrous trigones. Analyzing suture forces by region, those between the trigones exceeded those on the ring's posterior aspect. Among all studies, cyclic suture forces fell between $0.1-6.4 \mathrm{~N}$. Conclusions: Undersized mitral annuloplasty suture forces were measured for the first time in a healthy ovine model. These forces reveal trends according to both suture position and LVP. Future studies will utilize these devices to evaluate suture forces in the settings of functional mitral regurgitation and/or altered ring geometries. Findings will be quantitatively compared to the forces known to cause suture failure and tissue tearing. Collectively, these investigations will elucidate the mechanisms of ring dehiscence and inform next-generation ring designs.

\section{The Fate of the Aortic Root Following Implantation of Continuous Flow Left Ventricular Assist Device}

Sung Ho Jung, John M. Stulak, Jin-Oh Choi, Lyle D. Joyce, Richard C. Daly

Mayo Clinic, Rochester, MN, USA

Objective: The present study was performed to know whether the aortic root size increase or not during the left ventricular assist device (LVAD) support, so to confirm that the increase of aortic root size might contribute to the development of aortic regurgitation after LVAD implantation. Methods: Between February 2007 and December 2012, 212 patients underwent LVAD implantation. We only included the continuous flow type LVAD and excluded the patients with pulsatile LVAD, pre-implant aortic valve pathology more than mild, previous or concomitant aortic valve surgery, post implant surgery including the aortic valve or transplantation before 2 years postoperatively surgery, and less than 2 years follow up duration. We examined the degree of aortic valve regurgitation and echocardiographic measurements of the aortic valve annulus, aortic root and sinotubular junction in patients immediately post implant and after 2 years of LVAD support. Results: There were 43 patients who met criteria and in whom measurements were obtained. The median degree of aortic regurgitation increased from trivial immediately post implant to mild at 2 years. Median aortic valve annulus size (cm) remained stable (2.34 vs. $2.32, \mathrm{p}=0.55)$, as did the sinuses of Valsalva ( 3.49 vs. $3.47, \mathrm{p}=0.85)$ and cephalad to the sinotubular junction (2.93 vs. $2.97, \mathrm{p}=0.89$ ). Conclusions: We confirm the observation that aortic valve regurgitation progresses following long-term support with a continuous flow LVAD. The mechanism does not appear to be due to aortic annular and aortic root dilatation as we observe that the size of these structures remain stable after prolonged support.

\section{Surgical Relocation of the Papillary Muscles in Ischemic Mitral Regurgitation Resulted from Excessive Remodeling}

Sachiko Yamazaki, Hitoshi Yaku, Kiyoshi Doi, Kazunari Okawa, Tsunehisa Yamamoto, Suguru Ohira

Kyoto prefectural university of medicine, Kyoto, Japan

Objective: The purpose of the study is to determine whether relocation of papillary muscles (PMs) to the center of the annulus of the anterior mitral leaflet (AML) as an additional procedure to undersized mitral annuloplasty (uMAP) could prevent recurrence of ischemic mitral regurgitation (IMR). Methods: Between 2007 and 2012, 29 patients (mean age, 67.3 \pm 9.2 year) underwent relocation of PMs with CABG and uMAP. Relocation of PMs was indicated for patients with IMR when tethering height was $>5 \mathrm{~mm}$. Relocation of bilateral PMs was performed in patients with previous anterior myocardial infarction or dilated heart. The degree of MR and LV dimensions were assessed by echocardiography preoperatively, at discharge, and during follow-up (mean 23.7 month). Results: Preoperative MR was moderate in 15 patients, moderate to severe in 9 and severe in 5. The mean LVEF was $35 \pm 12 \%$ and the LVDd, LVDs were $60 \pm 7,50 \pm 9 \mathrm{~mm}$. Relocation of bilateral PMs was performed in 18 patients. There were 5 in-hospital deaths and 4 late deaths, none of them was related to MR. At discharge, postoperative TTE revealed zero or trace MR in all but 1 patient, who demonstrated moderate MR. During follow-up, 3 patient revealed moderate-severe MR 46.3 \pm 26.8 month after surgery. In remaining patients, valve function remained stable with MR less than mild degree, even though 4 patients showed further dilatation of LV during follow-up. Conclusions: Relocation of papillary muscles is simple and easily reproducible procedure. For patients even with continued LV remodeling, it may prevent and delay recurrence of MR.

\section{Autoantibodies to Oxidized LDL in Patients with Aortic Regurgitation: Association with Aortic Diameter Size}

Sara Shimoni, Iris Bar, Liaz Zilberman, Jacob George

Kaplan Hospital, Rehovot, Israel

Background: Aortic regurgitation (AR) is a condition associated with volume overload, causing left ventricular remodeling, eccentric left ventricular hypertrophy and eventually heart failure. Left ventricular remodeling associated with aortic regurgitation $(\mathrm{AR})$ is regulated by mechanical stress, neurohormonal activa- 
tion, inflammation and oxidative stress. Since anti-oxidized LDL (oxLDL) antibodies (Abs) are a measurable marker of oxidative stress, we hypothesized that an increased level of circulating oxLDL Abs may be related to remodeling of the left ventricle in patients with significant AR. Methods: We assessed IgG anti-oxLDL antibodies in 31 patients with significant aortic regurgitation and compared them to 30 patients with similar risk factors and no valvular disease. Antibodies to oxLDL were determined by ELISA. Results: The 2 groups had similar clinical characteristics. There was no difference in the level of anti-oxLDL antibodies between patients with AR and patients with no AR. However, in all patients and controls anti-oxLDL antibodies correlated positively with ascending aortic diameter $(r=0.32, p=0.016)$, and level of oxLDL antibodies was significantly higher in patients with $A A \geq 39 \mathrm{~mm}$. On multivariate analysis only white blood cell count and AA diameter were related to anti-oxLDL antibodies in all patients. Conclusions: We did not find a difference in the level of anti-oxLDL antibodies in patients with $\mathrm{AR}$ and controls, however there was a strong correlation between anti-oxLDL antibodies and ascending aortic diameter.

\section{8-Year Experience with the Carpentier-Edwards PERIMOUNT Standard Aortic Valve at a Single Japanese Center}

Koichi Arinaga, Koji Akasu, Hiriyuki Saisho, Toru Takaseya, Kumiko Wada, Hidetoshi Akasi, Hiroyuki Tanaka

Kurume University School of Medicine, Kurume, Japan

Background: The aim of this study was to evaluate the longterm results of the Carpentier-Edwards Pericardial (CEP) Standard valve at the aortic position. Methods: From January 1996 to December 2010, 275 patients who underwent aortic valve replacement with the CEP valve were enrolled. A $19-\mathrm{mm}$ valve was used in 42 patients, $21-\mathrm{mm}$ valve in 103 patients, $23-\mathrm{mm}$ valve in 97 patients, and $25-\mathrm{mm}$ valve in 33 patients. The early and late results were evaluated. Echocardiography was performed at follow-up in 191 of 270 late survivors for a mean duration of $6.3 \pm 3.2$ years. Left ventricular mass index (LVMI), mean pressure gradient (MPG), and effective orifice area index (EOAI) were measured. Results: There were 5 early deaths (early mortality rate, $1.82 \%)$. Among the 270 survivors, 255 (94.4\%) were followed-up for a mean duration of 7.4 years (range, 18 months to 17.2 years) for a total of 2037 patient-years. There were 80 (29.6\%) late deaths during the follow-up period. Cardiac death and valverelated death occurred in 35 patients. Actuarial survival at 10, and 15 years was $63.6 \%$ and $45.3 \%$, respectively. Thromboembolism was observed in 17 patients $(0.83 \%$ /patient-year $)$, prosthetic endocarditis in 3 patients $(0.15 \% / \mathrm{p}$-y), structural valve deterioration in 6 patients $(0.29 \% / \mathrm{p}-\mathrm{y})$, and reoperation in 9 patients $(0.44 \% / p-y)$. Actuarial freedom from thromboembolism, prosthetic endocarditis, structural valve deterioration and reoperation at 15 years was 83.7, 98.7, 85.1 and 77.9\%, respectively. Echocardiography revealed acceptable pressure gradients across the valve prosthesis in each valve size. MPG was $12.0 \pm 3.5 \mathrm{~mm} \mathrm{Hg}$ (19 mm), 9.9 $\pm 3.4 \mathrm{~mm} \mathrm{Hg}(21 \mathrm{~mm}), 11.4 \pm 6.4 \mathrm{~mm} \mathrm{Hg}(23 \mathrm{~mm})$, and $9.7 \pm 2.5 \mathrm{~mm} \mathrm{Hg}(25 \mathrm{~mm})$. EOAI was $0.83 \pm 0.13 \mathrm{~cm}^{2}(19 \mathrm{~mm})$, $1.00 \pm 0.20 \mathrm{~cm}^{2}(21 \mathrm{~mm}), 1.05 \pm 0.22 \mathrm{~cm}^{2}(23 \mathrm{~mm})$, and $1.11 \pm 0.21$ $\mathrm{cm}^{2}(25 \mathrm{~mm})$. LVMI decreased significantly in all valve sizes. Conclusion: The long-term results of CEP bioprosthesis in the aortic position were satisfactory. The hemodynamic performance of the CEP bioprosthesis is maintained even as late as 18 years after implantation.

\section{Tricuspid Valve Replacement in Patient with Congenital Heart Disease}

Sungkyu Cho, Yong Jin Kim, Eun Seok Choi, Woong-Han Kim, Jeong Ryul Lee

Seoul National University Hospital, Seoul, Republic of Korea

Objective: Tricuspid valve replacement in patient with congenital heart disease is not commonly performed. We reviewed to evaluate the long term outcomes of TVR in patients with congenital heart disease. Methods: From April, 1981 to September, 2012 tricuspid valve replacement was performed in 15 patients $(9 \mathrm{fe}-$ male) with congenital heart disease at our institution (total of 24 prostheses). The most frequent diagnosis was Ebstein anomaly in 8 patients. Results: Median age was 12.8 years (from 2.0 to 25.2 years) at the time of initial tricuspid valve replacement. 8 patients (median age: 9.1, from 2.0 to 17.0 years) underwent replacement with mechanical valves and 7 patients (median age 14.9: from 5.6 to 25.2 years) underwent replacement with tissue valves $(\mathrm{p}=0.95)$. One patient died early postoperatively due to acute myocardial failure. During a median follow up of 15.3 years (from 1.4 to 32.0 years), $50 \%$ of patients needed a re-replacement because of valve related complications (valve thrombosis or valve degeneration). The re-replacement rate by Kaplan-Meier log rank was higher in patients who underwent tricuspid valve replacement under 12 years old $(\mathrm{p}=0.01)$, but no significant re-replacement rate between mechanical valves and tissue valves $(\mathrm{p}=0.40)$. Conclusions: Tricuspid valve replacement in patients with congenital heart disease showed high incidence of re-replacement that might be unrelated to prosthesis material. The patients of young age at operation could be a risk factor for re-replacement of tricuspid valve replacement in this series.

\section{Surgical Results of Mitral Valve Repair for Congenital Mitral Valve Stenosis in Pediatric Patients}

\author{
Sungkyu Cho Woong-Han Kim, Eun Seok Choi, Jeong Ryul Lee, \\ Yong Jin Kim \\ Seoul National University Hospital, Seoul, Republic of Korea
}

Objective: Mitral valve repair has been performed congenital mitral valve stenosis in pediatric patients. But congenital mitral valve stenosis is a heterogeneous group of lesion and remained challenge. Methods: From March, 1999 to October, 2013 mitral valve repair was performed in 20 patients with congenital mitral 
valve stenosis. The median age was 10.3 month (from 22 days to 9.1 years) and the mean body weight was $7.7 \pm 4.1 \mathrm{~kg}$ at time of operation. Multiple level left-side heart obstruction was present in 9 patients (45\%). Severe mitral regurgitation present in one patient. Results: The main etiology of the mitral stenosis was supravalvular mitral ring in 7 patients, valvular in 5, parachute deformity of papillary muscles in 4 and other abnormal papillary muscles in 5 . Mean mitral valve pressure gradient improved from $10.5 \pm 4.1$ $\mathrm{mm} \mathrm{Hg}$ to $3.5 \pm 1.8 \mathrm{~mm} \mathrm{Hg}$ after mitral valve repair $(\mathrm{N}=16, \mathrm{p}<$ $0.0001)$. Mean follow up duration was $4.6+4.8$ years. One patient died postoperatively due to septic shock. Three patients required a second operation ( 1 for aortic stenosis, 2 for progressed mitral stenosis). Among them, two patients died, one patient due to $\mathrm{CPB}$ weaning failure and another patient due to multiple cerebral infarcts. At the last follow-up mean mitral valve pressure gradient was $4.7 \pm 3.2 \mathrm{~mm} \mathrm{Hg}$, and moderate or more mitral insufficiency was detected in 3 patients. At 10 years, survival by Kaplan-Meier was $81 \pm 10.0 \%$, and freedom from reoperation was $86.0 \pm 10.0 \%$. Conclusions: The pediatric patients who underwent mitral valve repair for congenital mitral stenosis showed good results. The follow-up echocardiography also revealed satisfactory results. Close follow-up is necessary to detect the development of postoperative mitral stenosis or regurgitation.

\section{Development and Characterization of a Decellularised Xenogeneic Mitral Valve Scaffold}

\author{
Marisa Granados, Lucrezia Morticelli, Pavel Yablonski, \\ Andres Hilfiker, Igor Tudorache, Sergei Cebotari, Axel Haverich, \\ Sotirios Korossis
}

Department of Cardiothoracic, Transplantation and Vascular Surgery Hannover Medical School, Hannover, Germany

Objective: The aim of this work was to develop and characterize a decellularised mitral valve scaffold for mitral valve replacement. Methods: Mitral valves from 6 month old pigs were disinfected, placed in hypotonic buffer and treated with SDS and sodium deoxycholate for 36 hours, followed by extensive washing cycles and nucleic acid digestion. Radial sections comprising annulus, leaflets, chordae tendinae, and papillary muscle were analyzed histologically by H\&E and DAPI staining, immunohistochemically by collagen IV, and by alpha-gal fluorescence staining. DNA was extracted from the annulus, anterior leaflet, chordae and papillary muscle, and quantified using a NanoDrop spectrophotometer. Sections of the treated leaflets were analyzed under transmission electron microscopy (TEM), whereas fresh and treated leaflet strips were subjected to uniaxial tensile loading to failure. Results: Following decellularisation, no cell nuclei were observed under H\&E or DAPI staining. There was also no change in the presence of collagen IV. The treatment resulted in a significant decrease of alpha-gal, as observed under fluorescence staining (fig. 1). DNA content was significantly reduced compared to the native tissue (99\%). TEM showed a cell-free decellularised tissue, with a conserved histoarchitecture. The decellularised tissue also demonstrated a grossly-maintained mechanical integrity. Conclusions: A protocol that effectively removed cells and DNA, whilst maintaining the native valve histoarchitecture and mechanical integrity was developed. Although some alpha-gal was still detectable after decellularisation, the significant reduction observed was encouraging. The presence of alpha-gal could potentially be overcome in the clinical setting by the use of alpha-gal knockout porcine tissue. However, analyzing the effect of decellularisation on alpha-gal in wild-type porcine tissue could provide an insight on whether other sugars, also potentially immunogenic, are removed. Future work will focus on optimizing the protocol in order to further decrease the alphagal content.
Fig. 1. H\&E, collagen IV and alpha-gal staining of fresh $(\mathbf{a}, \mathbf{b}, \mathbf{c})$ and decellularised leaflet $(\mathbf{d}, \mathbf{e}$, f) (for Abstract of Marisa Granados).
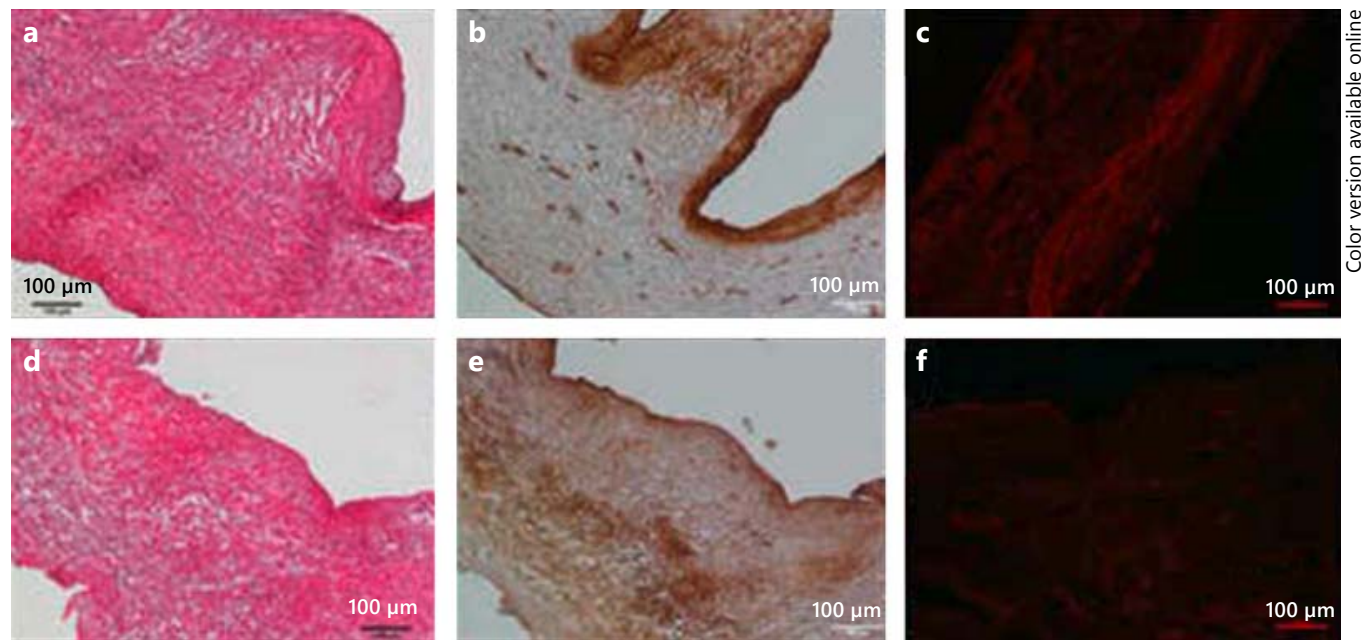


\section{Functional Tricuspid Regurgitation in Organic Mitral Regurgitation. New Insights into Right Ventricular Function}

\author{
Thierry Le Tourneau ${ }^{1}$, Caroline Cueff', Marjorie Richardson ${ }^{2}$, \\ Claude Hossein-Foucher ${ }^{2}$, Georges Fayad ${ }^{2}$, \\ Jean-Christian Roussel ${ }^{1}$, Jean-Noel Trochu ${ }^{1}$, André Vincentelli ${ }^{2}$ \\ ${ }^{1}$ Institut du Thorax, Nantes, France; ${ }^{2}$ University Hospital, \\ Lille, France
}

Objective: To assess the determinants of functional tricuspid regurgitation (TR) and its relations to right ventricular (RV) function in chronic organic mitral regurgitation (MR). Methods: Three-hundred twenty-five patients (63 112 years, 206 males) with organic MR (82\% degenerative etiology) referred to surgery and who underwent a preoperative gradation of TR were included in this study. Radionuclide angiography was carried out in 237 patients. Results: Fifty patients had a TR $\geq$ grade 2 . Patients with TR $\geq 2$ were older, had more AF ( 54 vs. $24 \%, \mathrm{p}<0.0001$ ) and were more symptomatic. Mean LV EF and RV EF were lower, and LV septal function and RV free wall function were impaired in those with TR $\geq 2$. By echocardiography, LV-RV, left and right atrial remodeling were worse, PASP was higher and inter ventricular systolic pressure was lower whereas the severity of MR was similar. RV S velocity was also significantly decreased. Ventricular function was stratified in normal RV-LV (Normal), isolated RV dysfunction (RVdysf, RV EF $\leq 35 \%$ ), isolated LV dysfunction (LVdysf, LV EF $<60 \%$ ), and biventricular impairment (BiV, LV EF $<60 \%$ and $\mathrm{RV}$ $\mathrm{EF} \leq 35 \%)$. TR $\geq 2$ was found mainly in either BiV (33\%) or LVdysf (22\%) but almost never in RVdysf (3\%) nor Normal (3\%) groups. In $\mathrm{BiV} \mathrm{TR} \geq 2$ was associated with overall impairment of the right heart while only the RA-annulus-RV base were enlarged in LVdysf. Finally RV EF alteration in RVdysf was likely linked mainly to compression and flattening of the RV by the severely enlarged LV owing to severe volume overload. Moreover, in RVdysf RV S wave velocity was not reduced and RV EF improvement after surgery was greater suggesting limited impairment of intrinsic myocardial function. These specific features probably explain the absence of TR in this subgroup of patients. Conclusions: In patients with organic MR referred to surgery $T R \geq 2$ is associated with the longstanding consequences of chronic organic MR. Tricuspid regurgitation occurs mainly in patients with $\mathrm{BiV}$ or LVdysf but is almost absent in RVdysf or Normal groups. Finally RVdysf group exhibits features suggesting a direct reversible effect of the magnitude of volume overload on the RV.

\section{Degradation of lodinated Cardiovascular Scaffolds Can Be Monitored In Vivo with CT Scanning}

\section{Hanna Talacua' ${ }^{1}$, Serge Söntjens ${ }^{2}$, Lex A. van Herwerden ${ }^{1}$, Tina Thakker ${ }^{3}$, Aryan Vink ${ }^{1}$, Aurelie Brizard ${ }^{4}$, Henk Janssen ${ }^{2}$, Jolanda Kluin ${ }^{1}$}

${ }^{1}$ UMC Utrecht, Utrecht, Netherlands; ${ }^{2}$ SyMo-Chem BV, Eindhoven, Netherlands; ${ }^{3}$ Eindhoven University of Technology, Eindhoven, Netherlands; ${ }^{4}$ Philips, Eindhoven, Netherlands

Objective: The ultimate goal of in situ tissue engineering is an autologous tissue engineered substitute, which is able to grow and remodel. The scaffold used should be biocompatible, non-thrombogenic, and biodegradable. To monitor degradation of biomaterials, non-invasive imaging is of great interest. The objective of this study is to investigate whether degradation of iodinated scaffolds can be monitored in vivo with CT scanning. Methods: Biopolymers were modified with iodinated compounds, to render them radiopaque. Upon degradation of the biopolymers, the iodine content slowly decreases, allowing the degradation state of the scaffold to be monitored in a non-invasive manner. The material was electrospun, leading to porous materials suitable for implantation. 24 Rats received an interposition graft with or without iodinated compounds ( $\mathrm{n}=12$ per group). To examine tissue formation and scaffold degradation grafts were explanted at different time points $(\mathrm{t}=1$ day, 2 weeks and 1 month; $\mathrm{n}=4$ per group). The explanted grafts were analyzed with (immuno)histochemistry and gel permeation chromatography (GPC). Before termination a CT scan with and without contrast was made. Results: Grafts without iodine were not visible on CT images. Iodinated grafts were detected on CT images. Over time a decrease in iodine volume was seen, corresponding with material degradation. The administered contrast was only visible in the lumen of the grafts. With histology a phased tissue formation was observed after 1 month in both groups. Cellular content and tissue formation was equal in both groups. Conclusion: While there is still tissue formation in vivo, the degradation of iodinated scaffolds can be monitored in vivo with CT scanning. This finding is of special interest for heart valve tissue engineering, because it is opening the possibility to monitor patients non-invasively. As a next step towards a clinical application of this method iodinated pulmonary heart valve prostheses will be implanted in a sheep model. 